\title{
Divergent Synthesis of Contorted Polycyclic Aromatics Containing Pentagons, Heptagon and/or Azulene
}

Taifeng Liu**, Chaoran Qi ${ }^{*}$, Qinghai Zhou*, Wenying Dai, Yuying Lan, Lanting Xu, Junxia Ren, Yangyang Pan, Lei Yang, Yongchao Ge, Yang-Kun Qu, Wenhua Li, Hexing Li and Shengxiong Xiao*

The Education Ministry Key Lab of Resource Chemistry, Joint International Research Laboratory of Resource Chemistry, Ministry of Education, Shanghai Key Laboratory of Rare Earth Functional Materials, College of Chemistry and Materials Science, Shanghai Normal University, Shanghai 200234, China.

\section{Table of Contents}

1 Characterizations and reaction conditions screening of the rearrangement..................................... 3

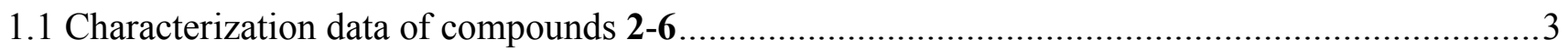

1.2 Reaction conditions screening of the rearrangement (Table S1) .........................................6

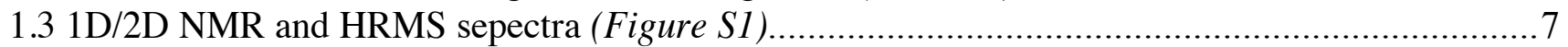

2 Crystal information of compounds 2-6 (Figure S2-S6, Table S2-S6) ............................................24

3 Optical and electronic spectra, frontier orbitals energies (Figure S7, Table S7) ............................... 34

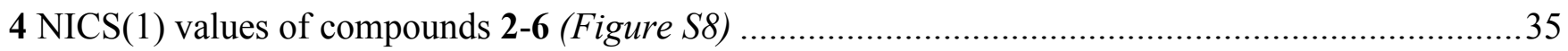

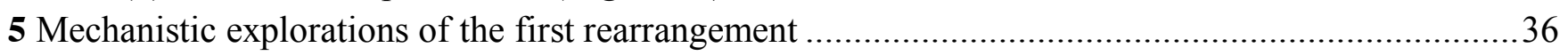

5.1 Reaction pathways of the first rearrangement (Figure S9-S12, Table S8) .................................... 36

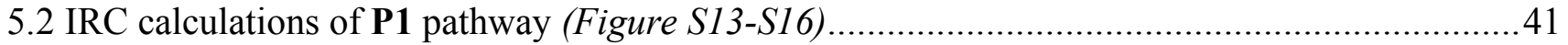

5.3 Calculations on the possible second rearrangement of 4 (Figure S17-S19, Table S9).................43

6 Calculations on the regioselective Scholl reaction from 4 to 3 (Figure $S 20-S 23$ ) ............................47

7 Calculations for the regioselective Scholl reaction from 2 to 5 (Table S10, Figure S24).................. 49

8 Calculations for the regioselective Mallory reaction from $\mathbf{2}$ to $\mathbf{6}$ (Figure S25, Table S11).................50

9 Frontier molecular orbital plots for compounds 2-6 (Figure S26-S29) ........................................52

10 Exclusion of other possible cyclizaion mechanisms and other rearrangement under TfOH/DDQ condition with the 2nd cyclization from 4 to 3 as computational model (Figure S30-S37, Table S12) ..54

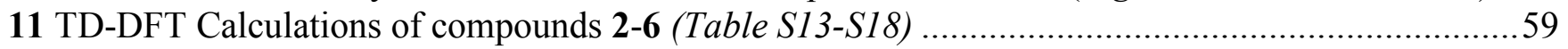

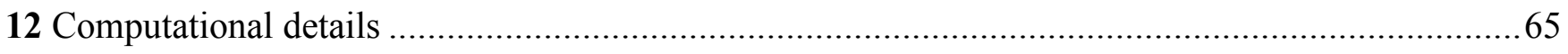

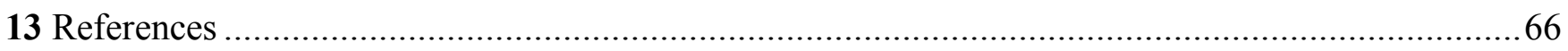

14 Cartesian coordinates with charge and multiplicity of all the optimized structures for calculations .. 67

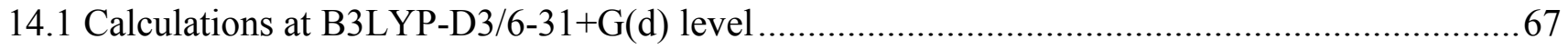

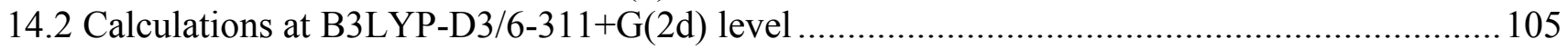




\section{General}

Unless otherwise noted, all materials and reagent including dry solvents were obtained from commercial suppliers such as Adamas-beta ${ }^{\circledR}$ and used without further purification. Unless otherwise noted, all work-up processes and purification procedures were carried out with reagent-grade solvents in air.

2,2'-bis(4,4,5,5-tetramethyl-1,3,2-dioxaborolan-2-yl)-1,1'-biphenyl was synthesized according to the procedure reported by Henry N. C. Wong. ${ }^{[1]}$ Compound 1 was synthesized according to the reported procedure. $^{[2]}$

Melting points were obtained on a NBchao melting point apparatus SGW X-4A (Made in Fo Shan, China). IR data was obtained on a Thermo Scientific apparatus Nicolet iS $10 .{ }^{1} \mathrm{H}$ and ${ }^{13} \mathrm{C}$ NMR spectra were obtained from a Bruker DRX400 (400 MHz) spectrometer at Shanghai Normal University, while 2D NMR spectra were obtained from a DMX600 (600 MHz) spectrometer at Shanghai Jiaotong University. The mass spectroscopic and HRMS data were obtained at the Shanghai Jiao Tong University mass spectrometry facility using a Bruker SolariX 7.0T FT ICR MS. Single crystal data was collected on an Bruker D8 Venture (Ga) diffractometer at Shanghai Institute of Organic Chemistry, Chinese Academy of Sciences. Absorption spectra were obtained on a Shimadzu UV 1800 UV-Vis spectrophotometer. Emission spectra were got on a EDINBURGH FLS980 spectrophotometer. Cyclic voltammetry $(\mathrm{CV})$ experiments were conducted with a CHI660E electrochemical workstation under an argon atmosphere. 


\section{Characterizations and reaction conditions screening of the rearrangement}

\subsection{Characterization data of compounds 2-6}

\section{Compound 2:}

To a Schlenk flask, 2,2'-bis(4,4,5,5-tetramethyl-1,3,2-dioxaborolan-2-yl)-1,1'-biphenyl (893.5 mg, 1.1 mmol, 2.2 eq.), 6,13-bis(dibromomethylene)-6,13-dihydropentacene 1 (310 mg, 0.5 mmol, 1.0 eq.), $\mathrm{K}_{2} \mathrm{CO}_{3}$ (552 mg, $4.0 \mathrm{mmol}, 8.0$ eq.), and $\mathrm{Pd}\left(\mathrm{PPh}_{3}\right)_{4}$ (58 mg, $0.05 \mathrm{mmol}, 0.1$ eq.) were added. This flask was evacuated and backfilled with $\mathrm{N}_{2}$ for three times. Then $20 \mathrm{~mL}$ of THF and $5 \mathrm{~mL}$ of water were bubbled with $\mathrm{N}_{2}$ for $1 \mathrm{~h}$ before this solution was added into the flask. The mixture was stirred and refluxed in an oil bath for $48 \mathrm{~h}$ under $\mathrm{N}_{2}$ atmosphere. Then it was cooled to room temperature and extracted with $\mathrm{CH}_{2} \mathrm{Cl}_{2}(50 \mathrm{~mL} \times 2)$, and washed with water $(100 \mathrm{~mL})$. The combined organic phase was added $50 \mathrm{~mL}$ of methanol, then $\mathrm{CH}_{2} \mathrm{Cl}_{2}$ was removed slowly via rotary evaporation. The precipitate was collected and washed with $20 \mathrm{~mL}$ of methanol, then dried at $60{ }^{\circ} \mathrm{C}$ under vacuum to afford $290 \mathrm{mg}$ (96\% yield) of compound $\mathbf{2}$ as a pale yellow solid. Compound $\mathbf{2}$ is stable under ambient conditions, and it can be dissolved in common organic solvents, such as $\mathrm{CH}_{2} \mathrm{Cl}_{2}$ and $\mathrm{CHCl}_{3}$ (approximately $1 \mathrm{mg}$ per $\mathrm{mL})$.

${ }^{1}$ H NMR $\left(400 \mathrm{MHz}, \mathrm{CDCl}_{3}\right) \delta \mathrm{ppm} 8.43(\mathrm{~s}, 4 \mathrm{H}), 8.13(\mathrm{~d}, J=8.0 \mathrm{~Hz}, 4 \mathrm{H}), 7.84(\mathrm{dd}, J=6.1,3.3 \mathrm{~Hz}, 4 \mathrm{H})$, $7.71(\mathrm{~d}, J=7.4 \mathrm{~Hz}, 4 \mathrm{H}), 7.54(\mathrm{dd}, J=6.3,3.2 \mathrm{~Hz}, 4 \mathrm{H}), 7.29$ (td, $J=7.5,0.9 \mathrm{~Hz}, 4 \mathrm{H}), 7.05-6.97$ (m, $4 \mathrm{H}) ;{ }^{13} \mathbf{C}$ NMR $\left(101 \mathrm{MHz}, \mathrm{CDCl}_{3}\right) \delta$ ppm 140.9, 138.4, 138.3, 137.2, 131.9, 131.0, 128.4, 128.2, 127.4, 126.9, 126.4, 125.2; MALDI-MS: calculated for $\mathrm{C}_{48} \mathrm{H}_{28}$ 604.2191, found [M] ${ }^{+}$604.2187.

\section{Compound 3: half-cyclized product after rearrangement}

Method 1 (one pot): Compound $2(120 \mathrm{mg}, 0.2 \mathrm{mmol})$ was dissolved in a mixture of $0.5 \mathrm{~mL}$ of TfOH and $100 \mathrm{~mL}$ of dry dichloromethane under $\mathrm{N}_{2}$ atmosphere. The mixture was stirred for $0.5 \mathrm{~h}$ at room temperature under $\mathrm{N}_{2}$ atmosphere. Then DDQ (227 mg, $1.0 \mathrm{mmol}, 5.0$ eq.) was added into the reaction solution, and this mixture was continually stirred for $15 \mathrm{~min}$. Then the reaction was quenched with saturated aqueous $\mathrm{NaHCO}_{3}$ solution (30 mL). Compound 3 was obtained as an orange solid (102 mg, 85\% yield) after purification via flash column chromatography (dichloromethane : hexane $=1: 2$ ).

Method 2 (4 as the reactant): Compound $4(120 \mathrm{mg}, 0.2 \mathrm{mmol})$ was dissolved in a mixture of TfOH $(0.5$ $\mathrm{mL})$, DDQ (227 mg, $1.0 \mathrm{mmol}, 5.0$ eq.) and dry dichloromethane (100 mL) under $\mathrm{N}_{2}$ atmosphere. The mixture was stirred for $15 \mathrm{~min}$ at room temperature under $\mathrm{N}_{2}$ atmosphere. Then the reaction was quenched with saturated aqueous $\mathrm{NaHCO}_{3}$ solution $(30 \mathrm{~mL})$. Compound $\mathbf{3}$ was obtained as an orange solid (114 mg, 95\% yield) after purification via flash column chromatography (dichloromethane : hexane $=1: 2$ ). 
M.p. $310-315{ }^{\circ} \mathrm{C} ;{ }^{1} \mathbf{H}$ NMR $\left(400 \mathrm{MHz}, \mathrm{CDCl}_{3}\right) \delta \mathrm{ppm} 9.09(\mathrm{~d}, J=8.1 \mathrm{~Hz}, 1 \mathrm{H}), 8.95(\mathrm{~d}, J=7.8 \mathrm{~Hz}, 1 \mathrm{H})$, 8.90-8.82 (m, 2H), $8.72(\mathrm{~d}, J=7.1 \mathrm{~Hz}, 1 \mathrm{H}), 8.56(\mathrm{~s}, 1 \mathrm{H}), 8.48(\mathrm{~d}, J=7.3 \mathrm{~Hz}, 1 \mathrm{H}), 8.43(\mathrm{~d}, J=8.1 \mathrm{~Hz}$, $1 \mathrm{H}), 8.30(\mathrm{~s}, 1 \mathrm{H}), 8.05(\mathrm{~d}, J=6.9 \mathrm{~Hz}, 1 \mathrm{H}), 7.99(\mathrm{~d}, J=7.4 \mathrm{~Hz}, 1 \mathrm{H}), 7.89$ (d, $J=8.0 \mathrm{~Hz}, 1 \mathrm{H}), 7.85-7.69$ $(\mathrm{m}, 7 \mathrm{H}), 7.56-7.46(\mathrm{~m}, 3 \mathrm{H}), 7.37(\mathrm{t}, J=7.3 \mathrm{~Hz}, 1 \mathrm{H}), 7.12(\mathrm{t}, J=7.6 \mathrm{~Hz}, 1 \mathrm{H}) ;{ }^{13} \mathbf{C} \mathbf{N M R}(101 \mathrm{MHz}$, $\left.\mathrm{CDCl}_{3}\right) \delta$ ppm 141.1, 139.2, 138.9, 137.3, 137.1, 135.8, 135.7, 135.5, 135.2, 135.0, 134.7, 134.4, 133.0, $133.0,132.8,132.6,132.6,132.5,131.7,131.0,130.7,130.4,129.9,129.5,128.7,128.4,128.1,128.1$, $127.9,127.6,127.5,127.4,127.3,127.2,127.2$, 126.8, 126.7, 126.5, 126.2, 124.6, 124.5, 123.7, 123.4, 121.2, 121.0, 119.2; IR (KBr, cm $\left.\mathrm{cm}^{-1}\right): \quad 3428,3051,2921,2850,1725,1600,1461,1441,1414,1262$, 1244, 1209, 1138, 789, 762, 730, 680, 615, 481, 460; MALDI-MS: calculated for $\mathrm{C}_{48} \mathrm{H}_{24} 600.1878$, found $[\mathrm{M}]^{+} 600.1875$.

\section{Compound 4: the rearrangement product}

Compound 2 (120 mg, $0.2 \mathrm{mmol}$ ) was dissolved in a mixture of $0.5 \mathrm{~mL}$ of $\mathrm{TfOH}$ and $100 \mathrm{~mL}$ of dry dichloromethane under $\mathrm{N}_{2}$ atmosphere. The mixture was stirred for $0.5 \mathrm{~h}$ at room temperature under $\mathrm{N}_{2}$ atmosphere. Then the reaction was quenched with saturated aqueous $\mathrm{NaHCO}_{3}$ solution $(30 \mathrm{~mL})$. Compound 4 was obtained as a white solid (108 $\mathrm{mg}$, 90\% yield) after purification via flash column chromatography (dichloromethane : hexane $=1: 3$ ).

M.p. $293-296{ }^{\circ} \mathrm{C} ;{ }^{1} \mathbf{H}$ NMR $\left(400 \mathrm{MHz}, \mathrm{CDCl}_{3}\right) \delta \mathrm{ppm} 8.75(\mathrm{~d}, J=8.2 \mathrm{~Hz}, 2 \mathrm{H}), 8.22(\mathrm{dd}, J=8.4,1.0 \mathrm{~Hz}$, $2 \mathrm{H}), 8.18(\mathrm{~s}, 2 \mathrm{H}), 8.10(\mathrm{~s}, 2 \mathrm{H}), 7.91-7.83(\mathrm{~m}, 4 \mathrm{H}), 7.66-7.60(\mathrm{~m}, 2 \mathrm{H}), 7.59$ (d, J = 7.4 Hz, 2H), 7.53$7.45(\mathrm{~m}, 6 \mathrm{H}), 7.44(\mathrm{~d}, J=7.9 \mathrm{~Hz}, 2 \mathrm{H}), 7.17(\mathrm{td}, J=7.5,1.0 \mathrm{~Hz}, 2 \mathrm{H}), 6.89(\mathrm{td}, J=7.8,1.1 \mathrm{~Hz}, 2 \mathrm{H}) ;{ }^{13} \mathrm{C}$ NMR $\left(101 \mathrm{MHz}, \mathrm{CDCl}_{3}\right) \delta$ ppm 144.27, 141.9, 140.7, 138.3, 134.5, 133.3, 132.9, 132.7, 132.2, 131.1, 131.0, 130.4, 128.4, 128.2, 128.0, 127.9, 127.0, 126.9, 126.7, 125.3, 124.6, 122.9, 119.7; IR (KBr, $\left.\mathrm{cm}^{-1}\right)$ : $3428,2963,2919,2850,1610,1443,1410,1261,1096,1022,863,801,745,704,672,620$;

MALDI-MS: calculated for $\mathrm{C}_{48} \mathrm{H}_{28} 604.2191$, found $[\mathrm{M}]^{+} 604.2192$.

\section{Compound 5: the half-cyclized anti- product}

To a two-neck flask, compound 2 (120 mg, $0.2 \mathrm{mmol}, 1.0$ eq.) was dissolved in super dry dichloroethane $(100 \mathrm{~mL})$ under $\mathrm{N}_{2}$ atmosphere. $\mathrm{FeCl}_{3}(325 \mathrm{mg}, 2.0 \mathrm{mmol}, 10.0$ eq. $)$ in $5 \mathrm{~mL}$ of nitromethane was added dropwise at $-20{ }^{\circ} \mathrm{C}$ (ice $/ \mathrm{NaCl}$ bath), then the mixture was stirred for 2 hours at $0{ }^{\circ} \mathrm{C}$ (ice/water bath) under $\mathrm{N}_{2}$ atmosphere. $\mathrm{MeOH}(5 \mathrm{~mL})$ was added to quench this Scholl reaction, followed by $100 \mathrm{~mL}$ of water. The organic phase was separated, dried over $\mathrm{Na}_{2} \mathrm{SO}_{4}$ and concentrated under reduced pressure. The product was purified by silica gel column chromatography (dichloromethane: petroleum ether $=1: 4, \mathrm{R}_{\mathrm{f}}=0.2$ ) to afford compound 5 as a yellow solid ( $82 \mathrm{mg}, 68 \%$ yield) and compound 6 as a yellow solid (30 mg, $25 \%$ yield). 
M.p. $275-279{ }^{\circ} \mathrm{C} ;{ }^{1} \mathbf{H}$ NMR $\left(400 \mathrm{MHz}, \mathrm{CDCl}_{3}\right) \delta \mathrm{ppm} 9.29(\mathrm{~d}, J=8.6 \mathrm{~Hz}, 2 \mathrm{H}), 9.09(\mathrm{~d}, J=8.4 \mathrm{~Hz}, 2 \mathrm{H})$, $8.93(\mathrm{~s}, 2 \mathrm{H}), 8.24(\mathrm{~d}, J=7.8 \mathrm{~Hz}, 2 \mathrm{H}), 8.13(\mathrm{~d}, J=7.0 \mathrm{~Hz}, 2 \mathrm{H}), 8.04(\mathrm{~d}, J=7.4 \mathrm{~Hz}, 2 \mathrm{H}), 7.99(\mathrm{~d}, J=7.5$ $\mathrm{Hz}, 2 \mathrm{H}), 7.90(\mathrm{dd}, J=8.4,7.1 \mathrm{~Hz}, 2 \mathrm{H}), 7.83(\mathrm{t}, J=7.1 \mathrm{~Hz}, 2 \mathrm{H}), 7.70(\mathrm{t}, J=7.4 \mathrm{~Hz}, 2 \mathrm{H}), 7.42(\mathrm{t}, J=7.3$ $\mathrm{Hz}, 2 \mathrm{H}), 7.22(\mathrm{t}, J=7.2 \mathrm{~Hz}, 2 \mathrm{H}) ;{ }^{13} \mathbf{C} \mathbf{N M R}\left(101 \mathrm{MHz}, \mathrm{CDCl}_{3}\right) \delta \mathrm{ppm} 141.4,138.7,137.2,134.7,133.1$, 133.0, 131.4, 131.0, 131.0, 130.4, 129.3, 128.9, 128.6, 128.4, 128.3, 127.7, 127.6, 127.6, 127.5, 127.1, 126.9, 124.0, 121.4, 119.5; IR (KBr, cm $\left.\mathrm{cm}^{-1}\right): 3439,3053,1624,1597,1475,1487,1444,1349,1298$, 1214, 1157, 1137, 1037, 1017, 954, 906, 837, 776, 751, 729, 671, 634, 597, 480; MALDI-MS: calculated for $\mathrm{C}_{48} \mathrm{H}_{24} 600.1878$, found $[\mathrm{M}]^{+} 600.1877$.

\section{Compound 6: the half-photocyclized syn-product}

To a quartz photoreactor, compound $2(120 \mathrm{mg}, 0.2 \mathrm{mmol})$, iodine (51 $\mathrm{mg}, 0.4 \mathrm{mmol})$ and dry toluene $(100 \mathrm{~mL})$ was added. The resultant purple solution was photo-irradiated using a $500 \mathrm{~W}$ medium-pressure mercury lamp for 12 hours at ambient temperature. Then, toluene was removed by rotator evaporation. The residue was purified by silica gel column chromatograph (dichloromethane: petroleum ether $\left.=1: 4, \mathrm{R}_{\mathrm{f}}=0.2\right)$ to afford compound $\mathbf{6}$ as a yellow solid (114 $\mathrm{mg}, 95 \%$ yield).

M.p. $270-275{ }^{\circ} \mathrm{C} ;{ }^{1} \mathbf{H}$ NMR $\left(400 \mathrm{MHz}, \mathrm{CDCl}_{3}\right) \delta \mathrm{ppm} 9.12(\mathrm{~s}, 2 \mathrm{H}), 8.93(\mathrm{dd}, J=6.0,3.4 \mathrm{~Hz}, 2 \mathrm{H}), 8.84$ $(\mathrm{d}, J=8.4 \mathrm{~Hz}, 2 \mathrm{H}), 8.72(\mathrm{~d}, J=7.8 \mathrm{~Hz}, 2 \mathrm{H}), 8.07$ (d, $J=6.9 \mathrm{~Hz}, 2 \mathrm{H}), 8.04(\mathrm{~d}, J=7.4 \mathrm{~Hz}, 2 \mathrm{H}), 7.93$ (dd, $J=6.1,3.2 \mathrm{~Hz}, 2 \mathrm{H}), 7.80(\mathrm{dd}, J=8.1,7.2 \mathrm{~Hz}, 2 \mathrm{H}), 7.69(\mathrm{dd}, J=6.2,3.2 \mathrm{~Hz}, 2 \mathrm{H}), 7.56(\mathrm{dd}, J=6.3,3.1$ $\mathrm{Hz}, 2 \mathrm{H}), 7.45(\mathrm{t}, J=7.3 \mathrm{~Hz}, 2 \mathrm{H}), 7.36(\mathrm{t}, J=7.6 \mathrm{~Hz}, 2 \mathrm{H}) ;{ }^{13} \mathbf{C ~ N M R}\left(101 \mathrm{MHz}, \mathrm{CDCl}_{3}\right) \delta \mathrm{ppm} 140.9$, 138.8, 137.0, 132.9, 132.4, 131.0, 130.1, 130.0, 129.5, 129.3, 128.7, 128.1, 127.9, 127.6, 126.9, 126.6, 126.2, 125.1, 124.0, 121.4, 119.5; IR (KBr, cm $\left.{ }^{-1}\right): 3427,3052,2961,2921,2851,1645,1480,1442$, 1411, 1261, 1096, 1025, 915, 866, 801, 768, 755, 697, 672;MALDI-MS: calculated for $\mathrm{C}_{48} \mathrm{H}_{24}$ 600.1878 , found $[\mathrm{M}]^{+} 604.1877$. 


\subsection{Reaction conditions screening of the rearrangement (Table S1)}

Table S1. Reaction conditions of precursor 2 in anhydrous solvent under $\mathrm{N}_{2}$ atmosphere.

\begin{tabular}{|c|c|c|c|c|c|c|c|c|}
\hline Entry & $\begin{array}{c}\text { Catalyst } \\
\text { /eq. }\end{array}$ & $\begin{array}{l}\text { Solvent } \\
\text { /Additive }\end{array}$ & $\begin{array}{c}\text { Temp. } \\
/^{\circ} \mathrm{C}\end{array}$ & $\begin{array}{c}\text { Time } \\
\text { / h }\end{array}$ & $\begin{array}{c}3 \\
/ \%\end{array}$ & $\begin{array}{c}4 \\
/ \%\end{array}$ & $\begin{array}{c}5 \\
/ \%\end{array}$ & $\begin{array}{c}6 \\
/ \%\end{array}$ \\
\hline 1 & $\mathrm{I}_{2} / 5.0$ & Toluene/- & $25(h v)$ & 6 & - & - & - & 95 \\
\hline 2 & $\mathrm{HCOOH} / 50.0$ & $\mathrm{DCM} /-$ & 25 to 45 & 24 & - & - & - & - \\
\hline 3 & $\mathrm{H}_{3} \mathrm{PO}_{4} / 50.0$ & $\mathrm{DCM} /-$ & 25 to 45 & 24 & - & - & - & - \\
\hline 4 & $\mathrm{CF}_{3} \mathrm{COOH} / 50.0$ & $\mathrm{DCM} /-$ & 25 to 45 & 24 & - & - & - & - \\
\hline 5 & $\mathrm{CH}_{3} \mathrm{SO}_{3} \mathrm{H} / 50.0$ & $\mathrm{DCM} /-$ & 25 to 45 & 24 & - & - & - & - \\
\hline 6 & $\mathrm{H}_{2} \mathrm{SO}_{4} / 50.0$ & $\mathrm{DCM} /-$ & 25 to 45 & 24 & - & - & - & - \\
\hline 7 & $\mathrm{AlCl}_{3} / 5.0$ & benzene/- & -20 to 25 & 2 & - & - & - & - \\
\hline 8 & $\mathrm{FeCl}_{3} / 5.0$ & $\mathrm{DCM} /-$ & -20 to 0 & 2 & - & - & 20 & 5 \\
\hline 9 & $\mathrm{FeCl}_{3} / 5.0$ & $\mathrm{DCE} /-$ & -20 to 0 & 2 & - & - & 68 & 25 \\
\hline 10 & $\mathrm{Sc}\left(\mathrm{CF}_{3} \mathrm{SO}_{3}\right)_{3} / 5.0$ & Toluene/- & 110 & 12 & - & - & - & - \\
\hline 11 & $\mathrm{Sc}\left(\mathrm{CF}_{3} \mathrm{SO}_{3}\right)_{3} / 5.0$ & Toluene/DDQ & 110 & 12 & - & - & - & - \\
\hline 12 & $\mathrm{CF}_{3} \mathrm{SO}_{3} \mathrm{H} / 1.0$ & $\mathrm{DCM} /-$ & 25 & 6 & - & 70 & - & - \\
\hline 13 & $\mathrm{CF}_{3} \mathrm{SO}_{3} \mathrm{H} / 5.0$ & $\mathrm{DCM} /-$ & 25 & 6 & - & 82 & - & - \\
\hline 14 & $\mathrm{CF}_{3} \mathrm{SO}_{3} \mathrm{H} / 30.0$ & $\mathrm{DCM} /-$ & 25 & 0.5 & - & 90 & - & - \\
\hline 15 & $\mathrm{CF}_{3} \mathrm{SO}_{3} \mathrm{H} / 30.0$ & $\mathrm{DCM} /-$ & 45 & 12 & - & - & - & - \\
\hline 16 & $\mathrm{CF}_{3} \mathrm{SO}_{3} \mathrm{H} / 30.0$ & $\mathrm{DCE} /-$ & 85 & 5 & - & - & - & - \\
\hline 17 & $\begin{array}{l}\mathrm{CF}_{3} \mathrm{SO}_{3} \mathrm{H} / 30.0 \\
\text { Then DDQ/5.0 }\end{array}$ & $\mathrm{DCM} /-$ & 25 & $\begin{array}{c}0.5 \\
0.25\end{array}$ & 85 & - & - & - \\
\hline
\end{tabular}

Note: DCM is dichloromethane; DCE is 1,2-dichloroethane, DDQ is 2,3-dichloro-5,6-dicyanobenzoquinone. 


\subsection{D/2D NMR and HRMS sepectra (Figure S1)}
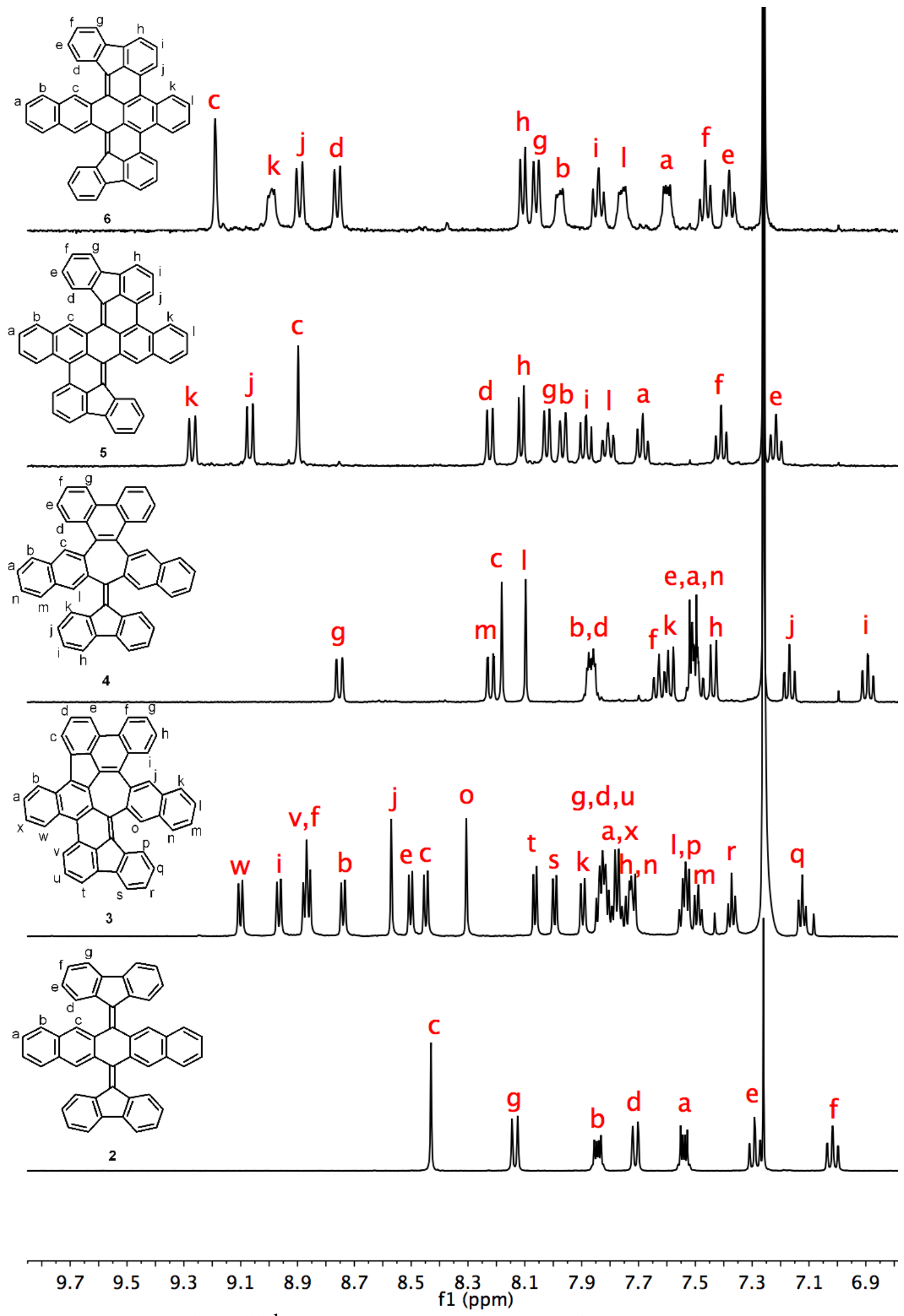

Figure S1. ${ }^{1} \mathrm{H}$ NMR and proton assignments of compounds 2-6. 


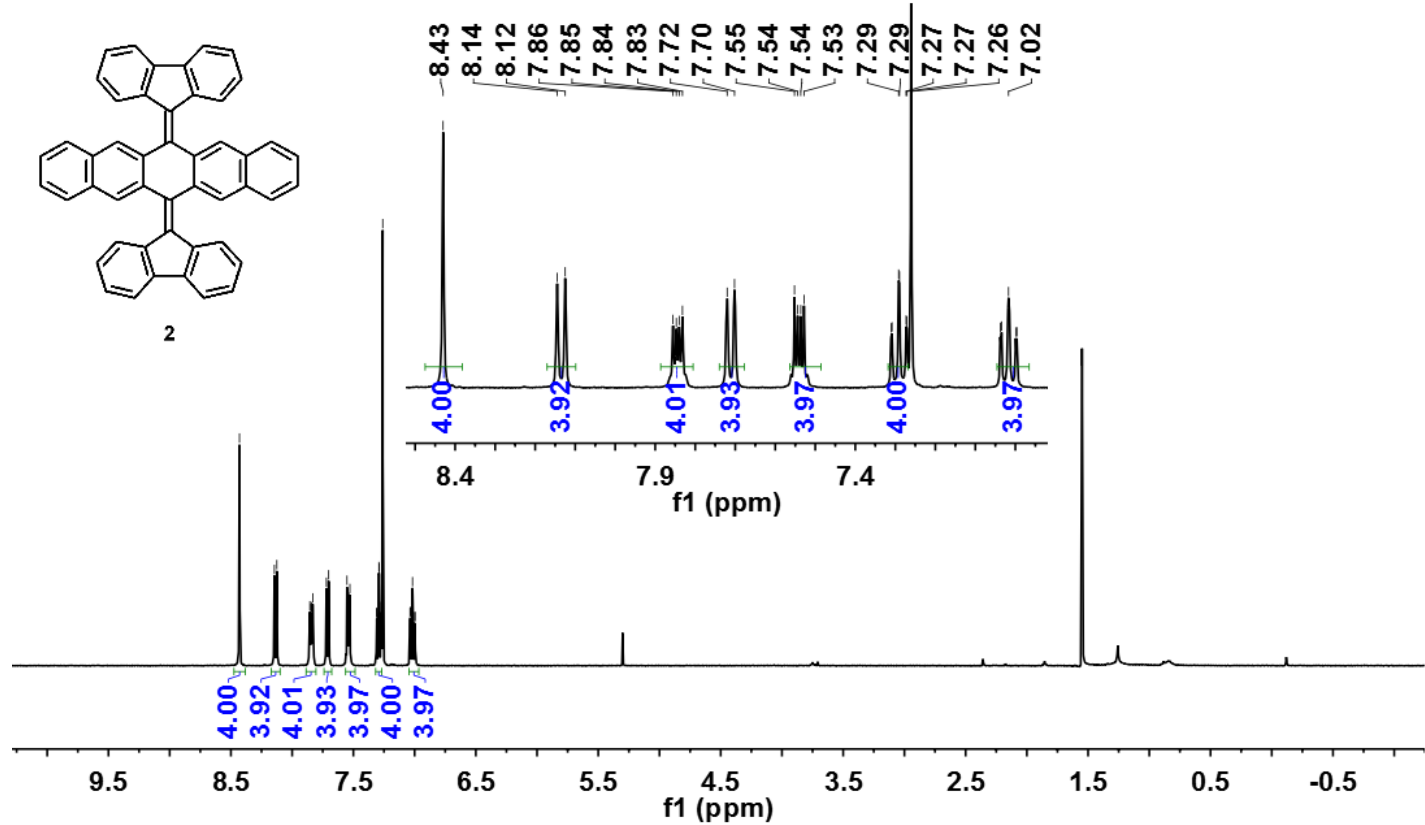

${ }^{1} \mathrm{H}$ NMR spectrum of compound $2\left(400 \mathrm{MHz} \mathrm{CDCl}_{3}, 300 \mathrm{~K}\right)$

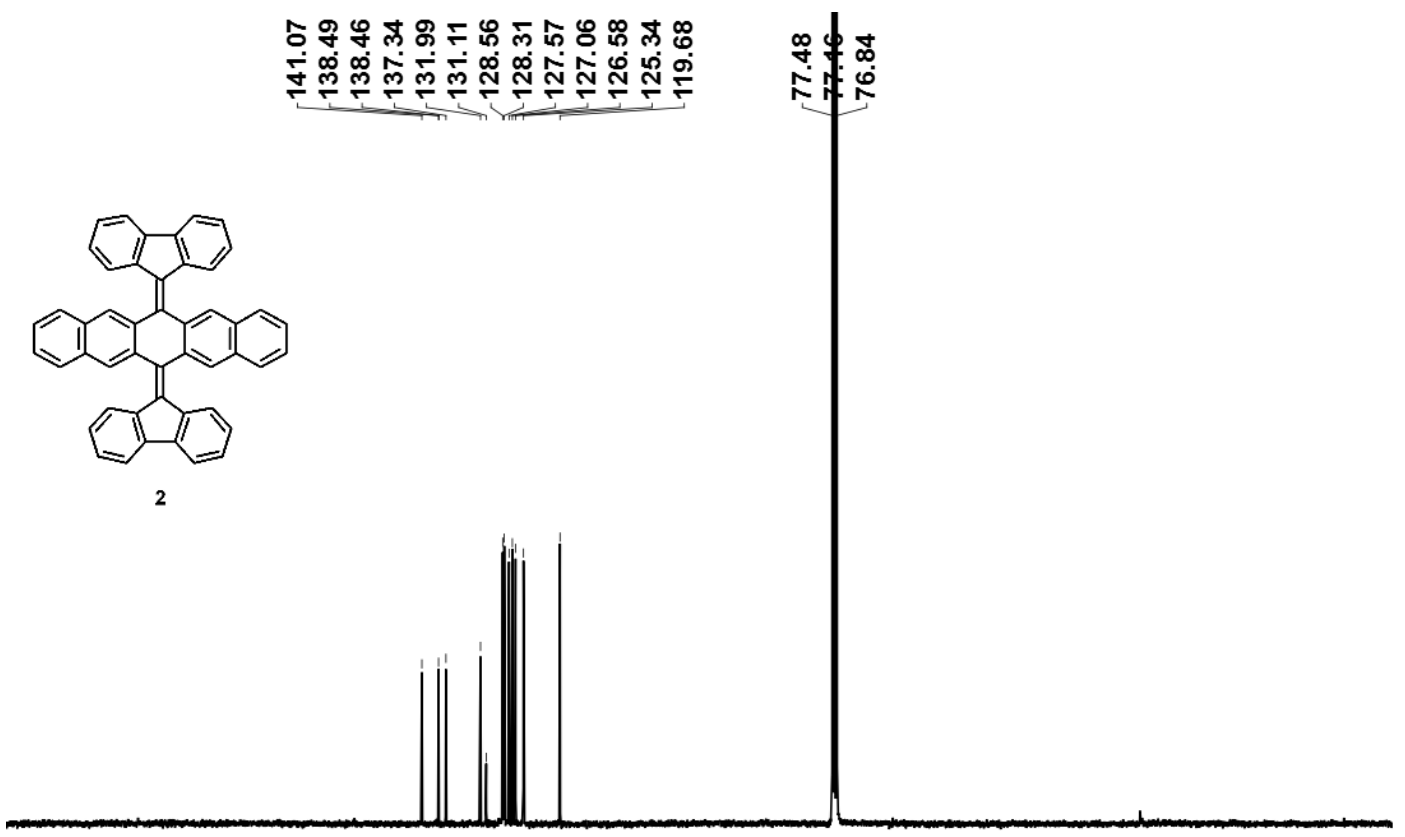

190 170 150 130 $110 \underset{f 1(p p m)}{90}$

${ }^{13} \mathrm{C}$ NMR spectrum of compound $2\left(101 \mathrm{MHz}, \mathrm{CDCl}_{3}, 300 \mathrm{~K}\right)$ 


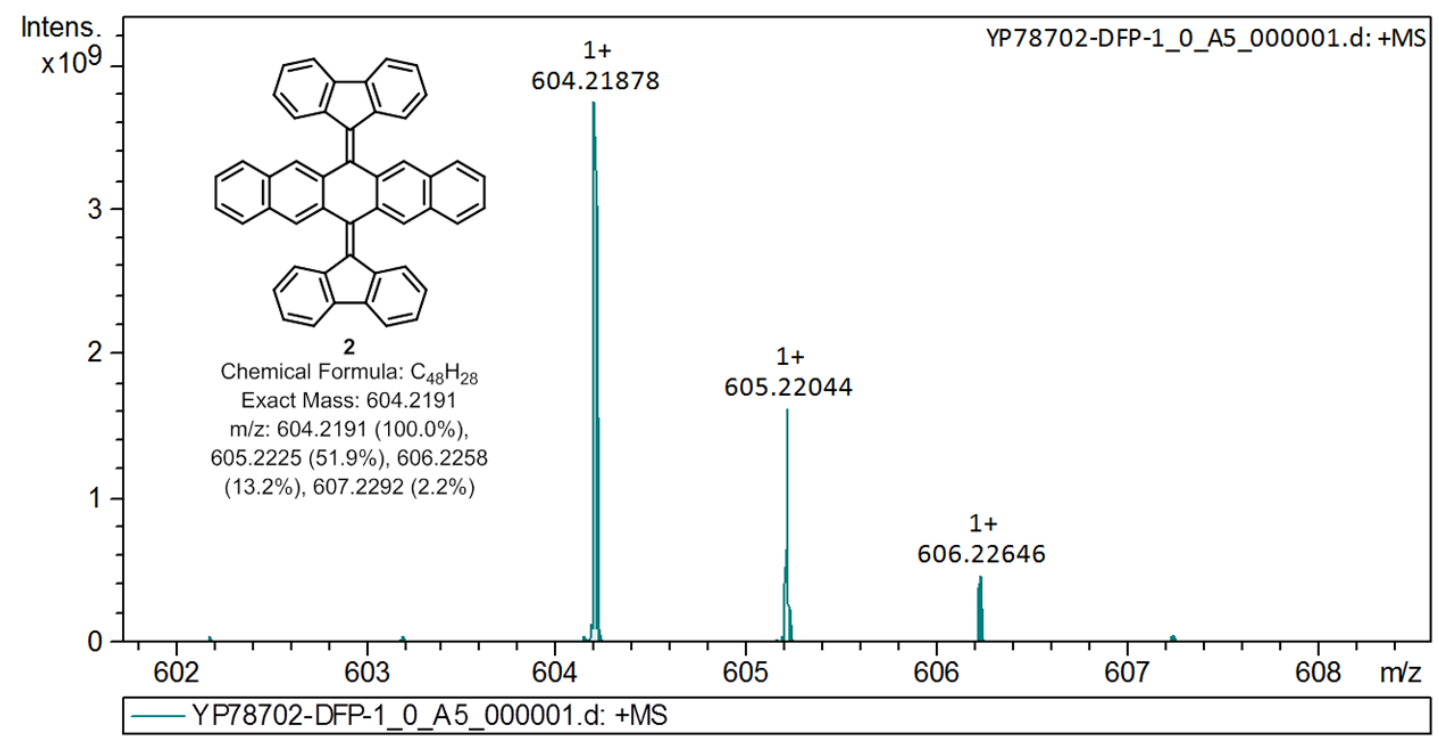

MALDI-MS of compound 2

웅요 \&

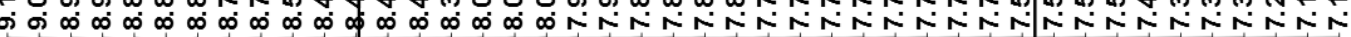
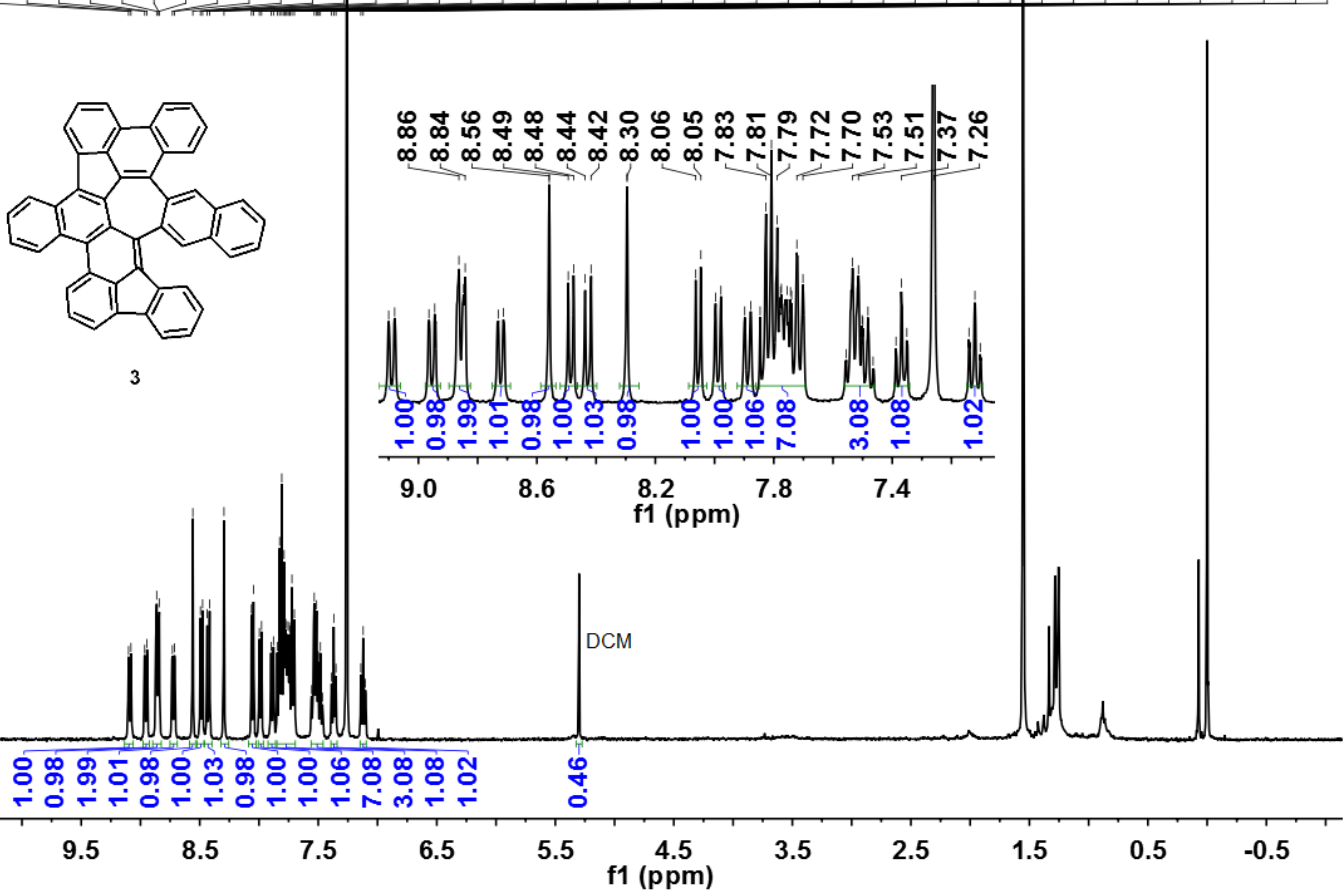

${ }^{1} \mathrm{H}$ NMR spectrum of compound $3\left(400 \mathrm{MHz} \mathrm{CDCl}_{3}, 300 \mathrm{~K}\right)$ 


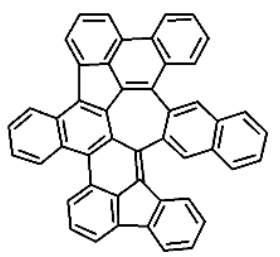

3
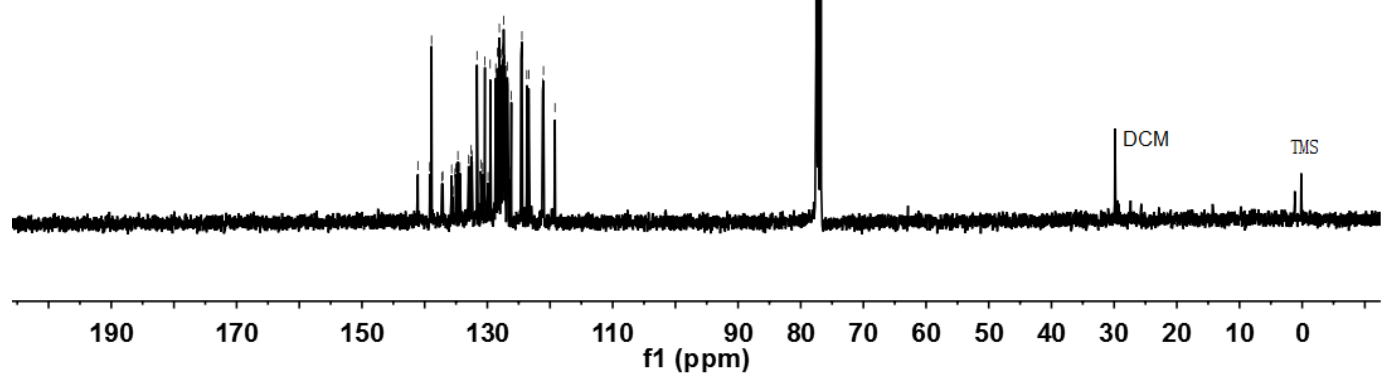

${ }^{13} \mathrm{C}$ NMR spectrum of compound $3\left(101 \mathrm{MHz}, \mathrm{CDCl}_{3}, 300 \mathrm{~K}\right)$

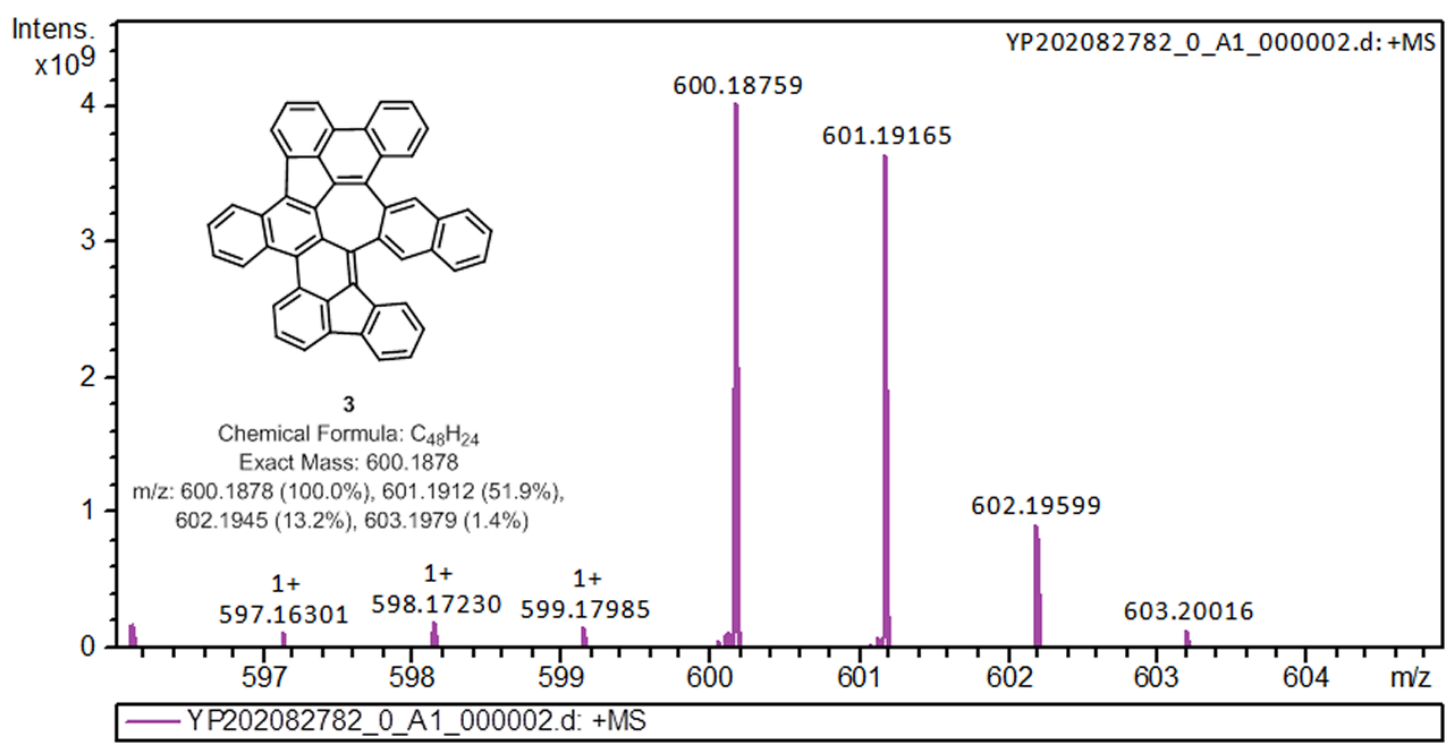

MALDI-MS of compound 3 


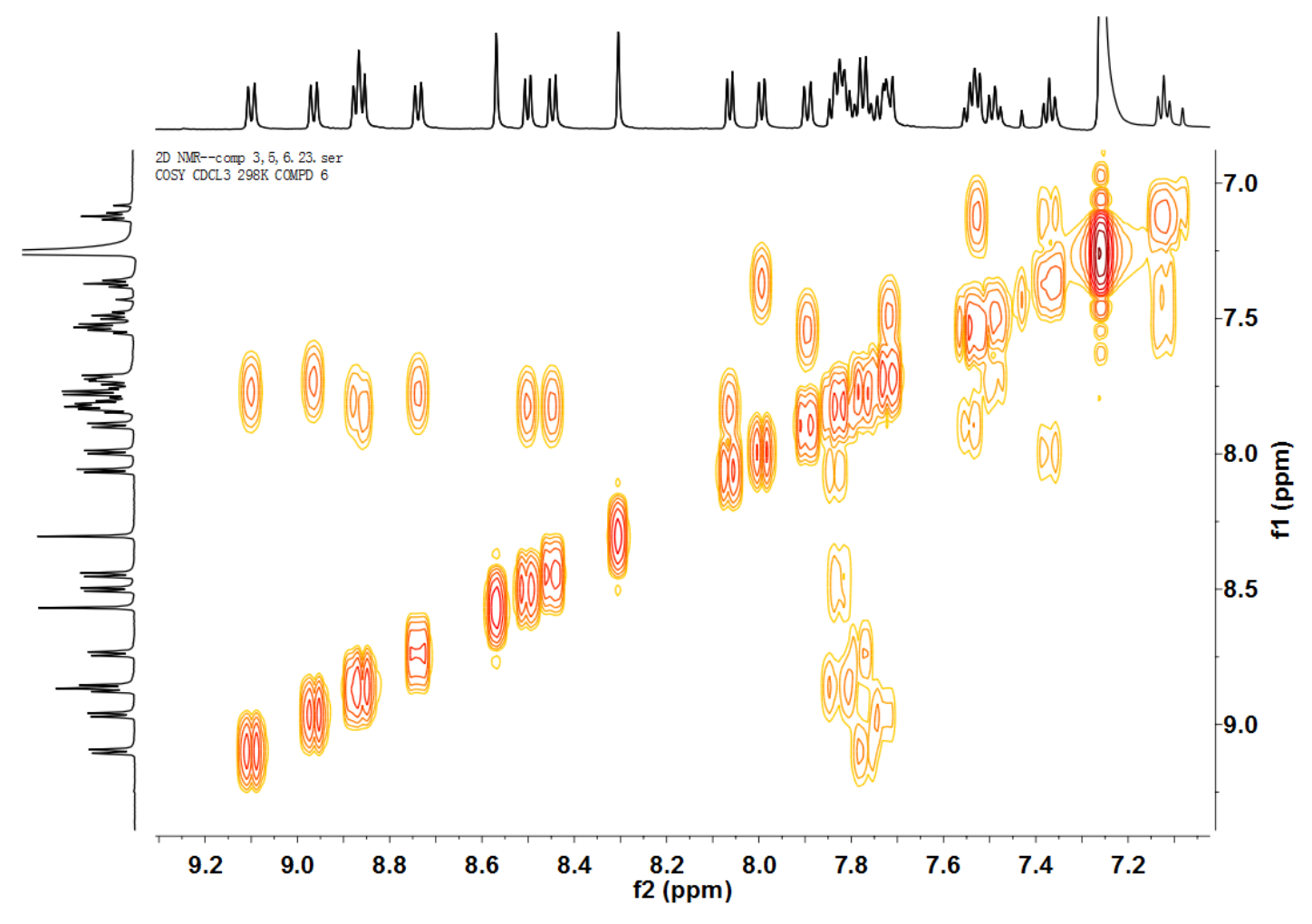

COSY spectrum of compound $3\left(600 \mathrm{MHz}, \mathrm{CDCl}_{3}, 298 \mathrm{~K}\right.$ )

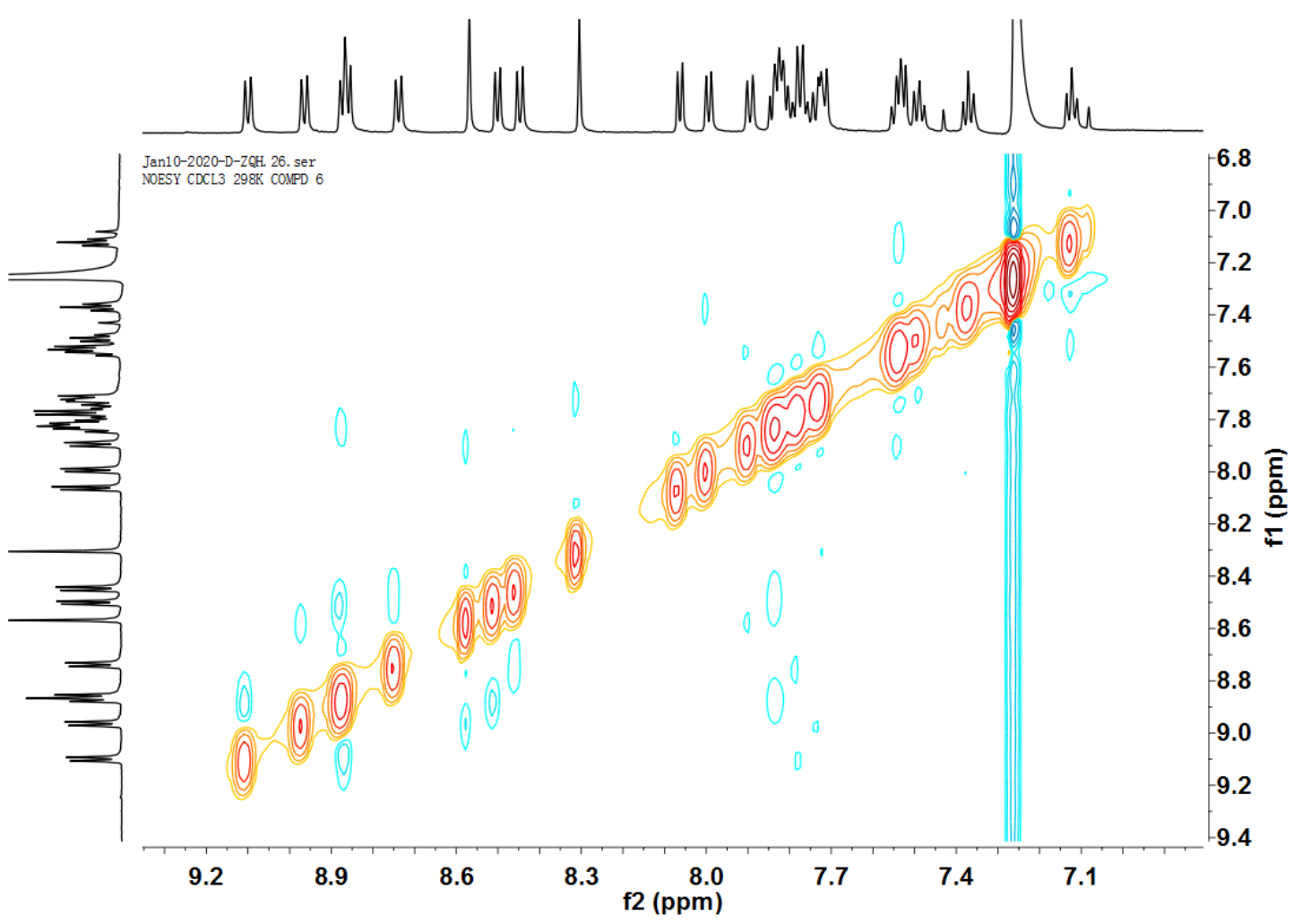

NOESY spectrum of compound $3\left(600 \mathrm{MHz}, \mathrm{CDCl}_{3}, 298 \mathrm{~K}\right)$ 


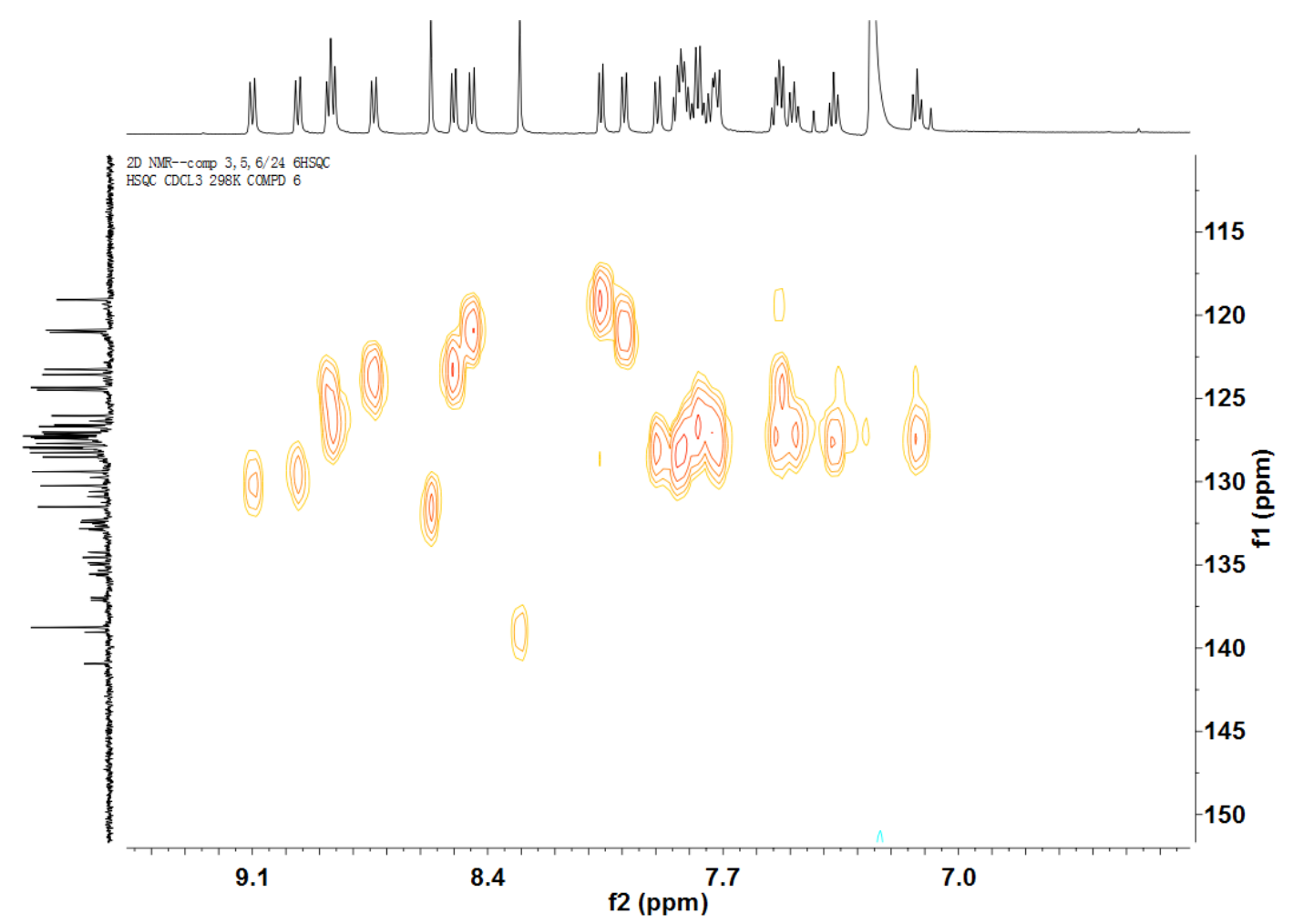

HSQC spectrum of compound $3\left(600 \mathrm{MHz}, \mathrm{CDCl}_{3}, 298 \mathrm{~K}\right)$

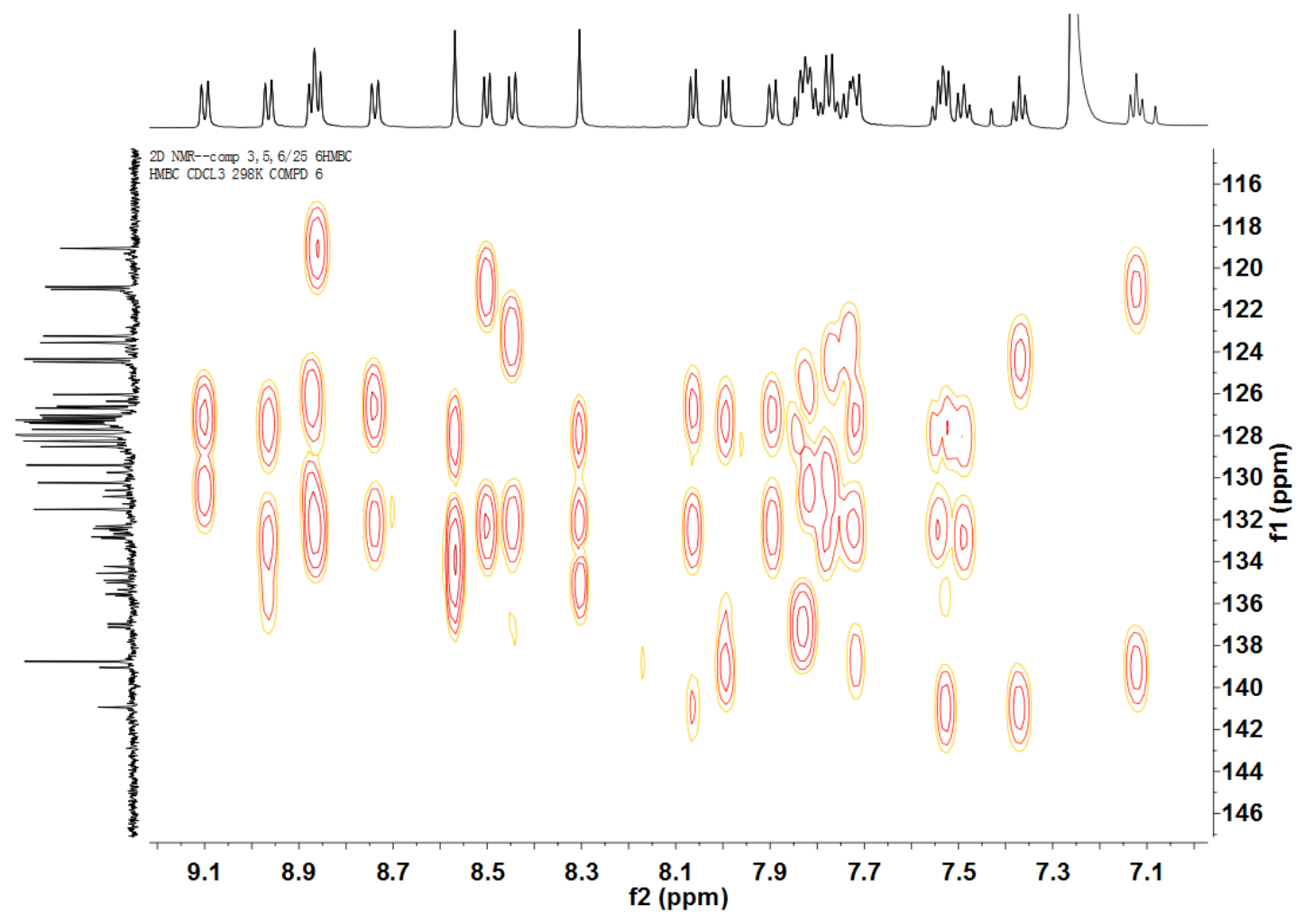

HMBC spectrum of compound $3\left(600 \mathrm{MHz}, \mathrm{CDCl}_{3}, 298 \mathrm{~K}\right)$ 


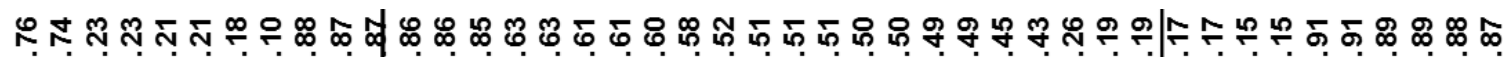

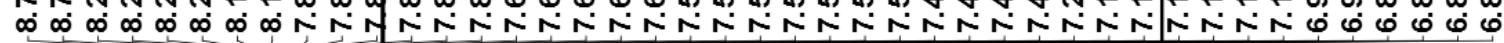

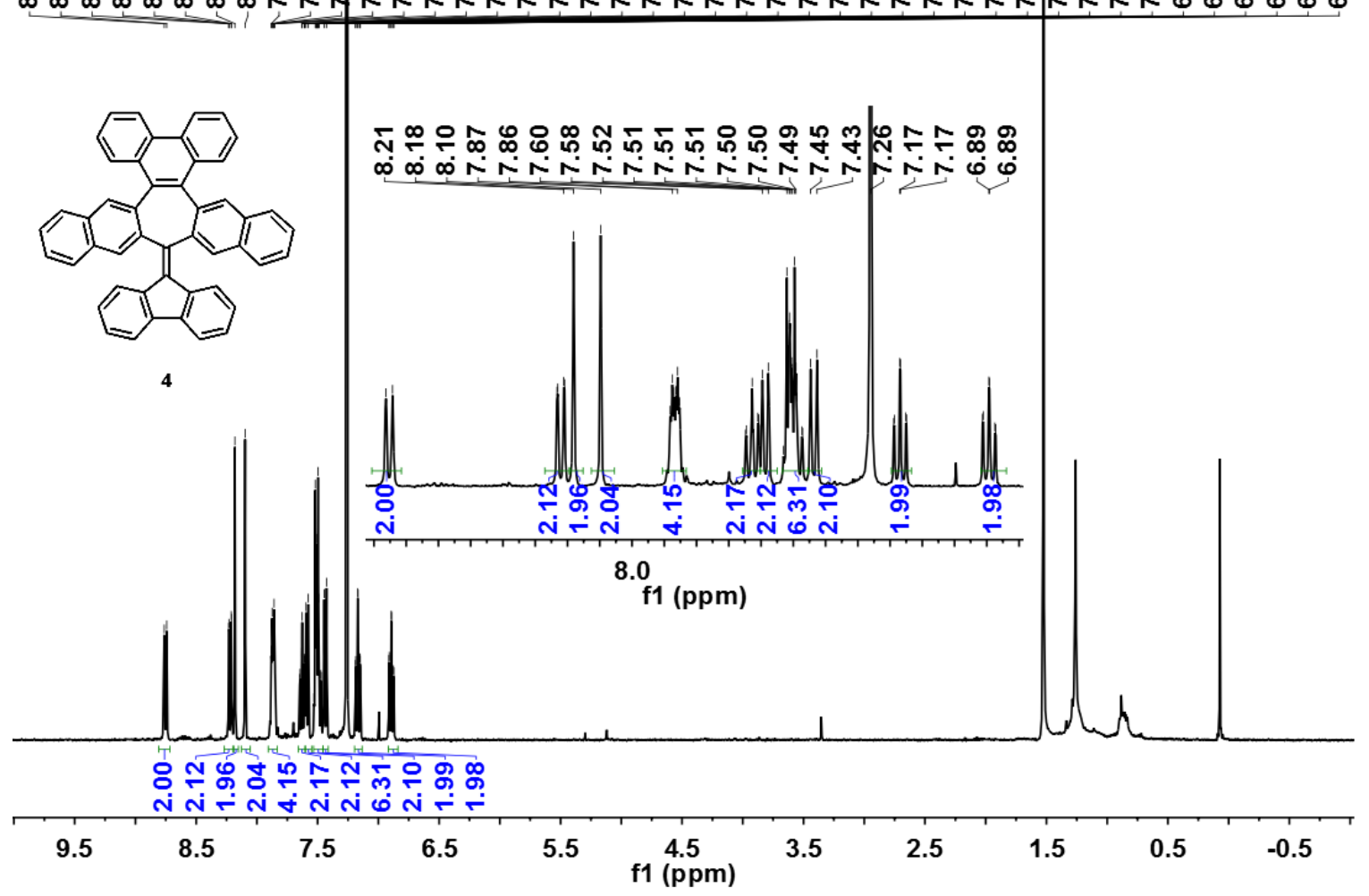

${ }^{1} \mathrm{H}$ NMR spectrum of compound $4\left(400 \mathrm{MHz}, \mathrm{CDCl}_{3}, 300 \mathrm{~K}\right)$

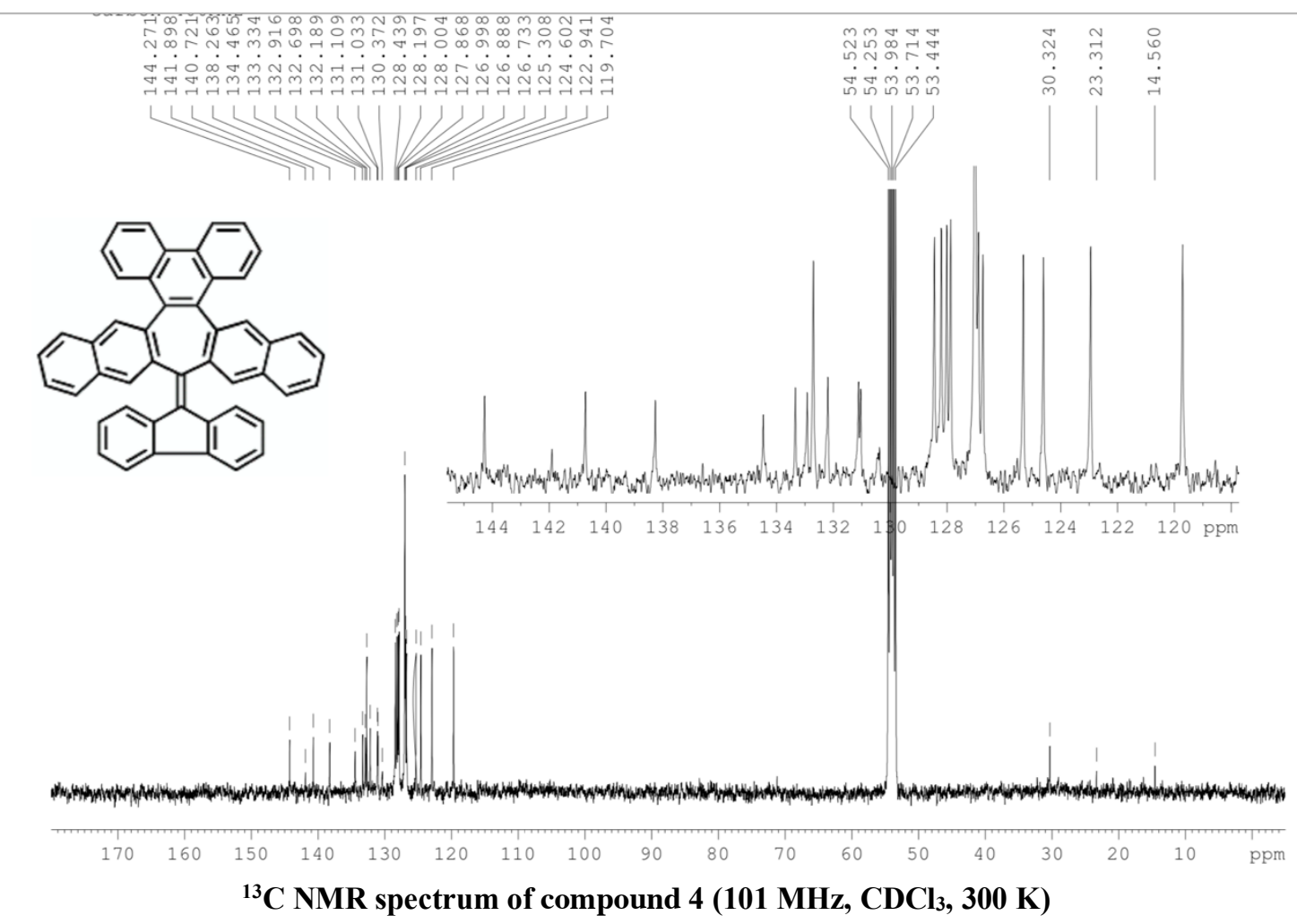




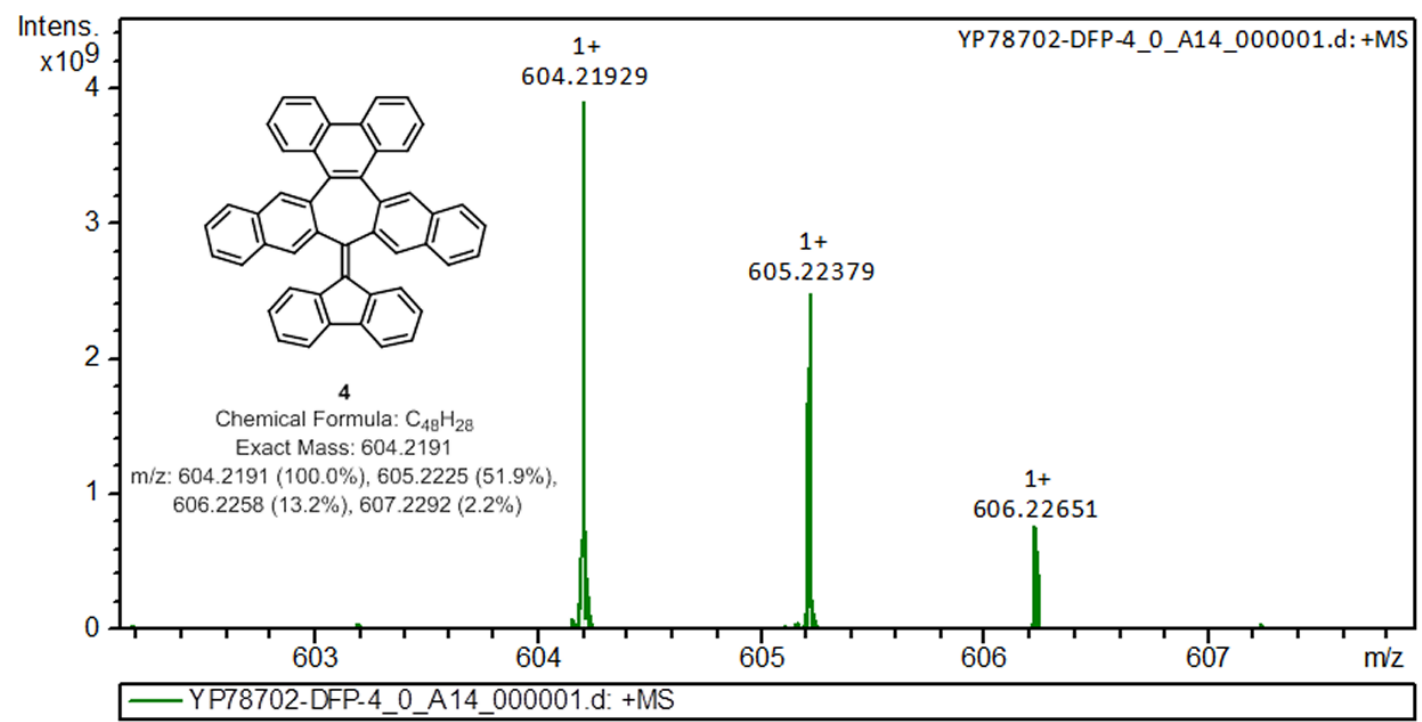

MALDI-MS of compound 4

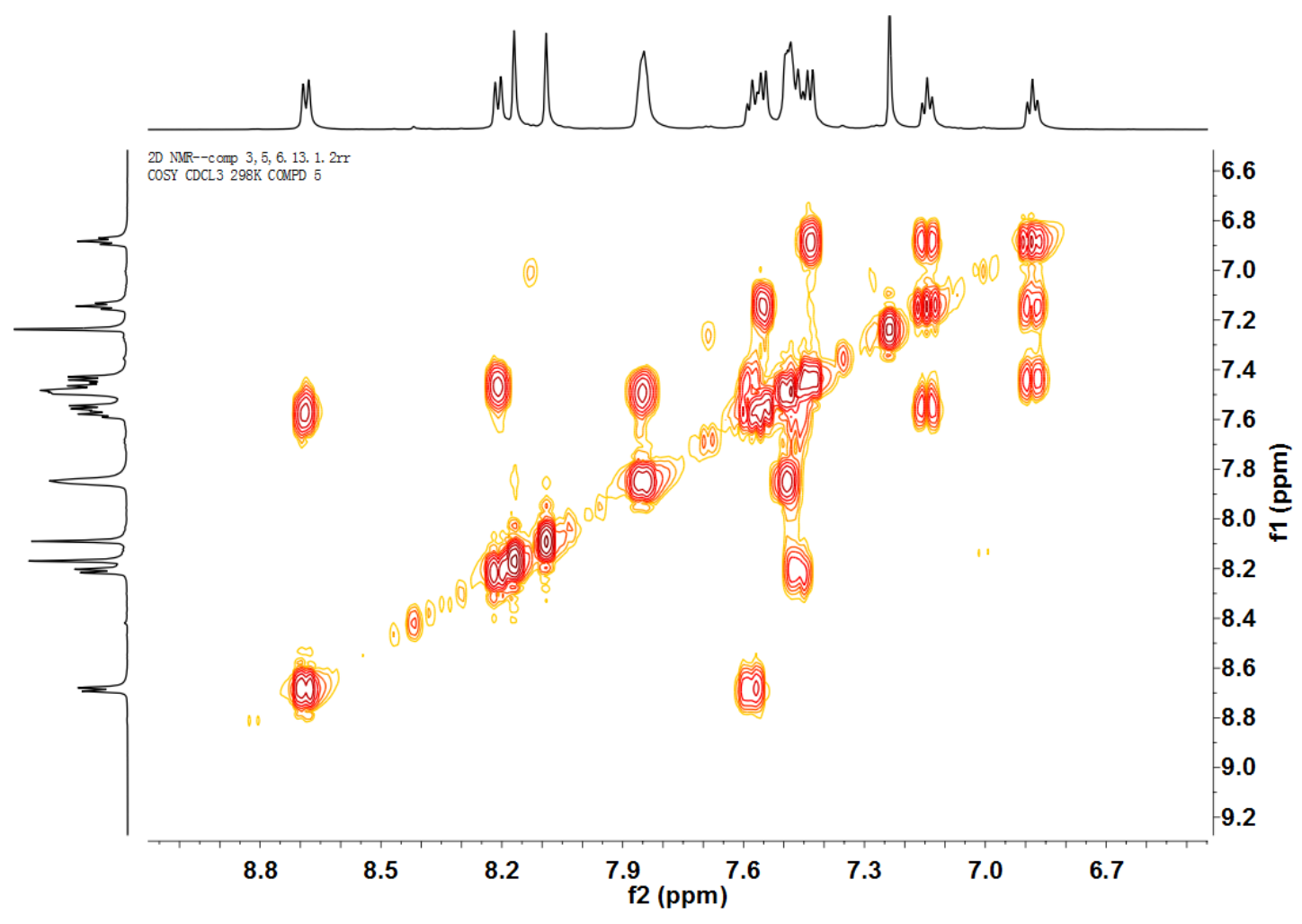

COSY spectrum of compound 4 (600 $\left.\mathrm{MHz}, \mathrm{CDCl}_{3}, 298 \mathrm{~K}\right)$ 


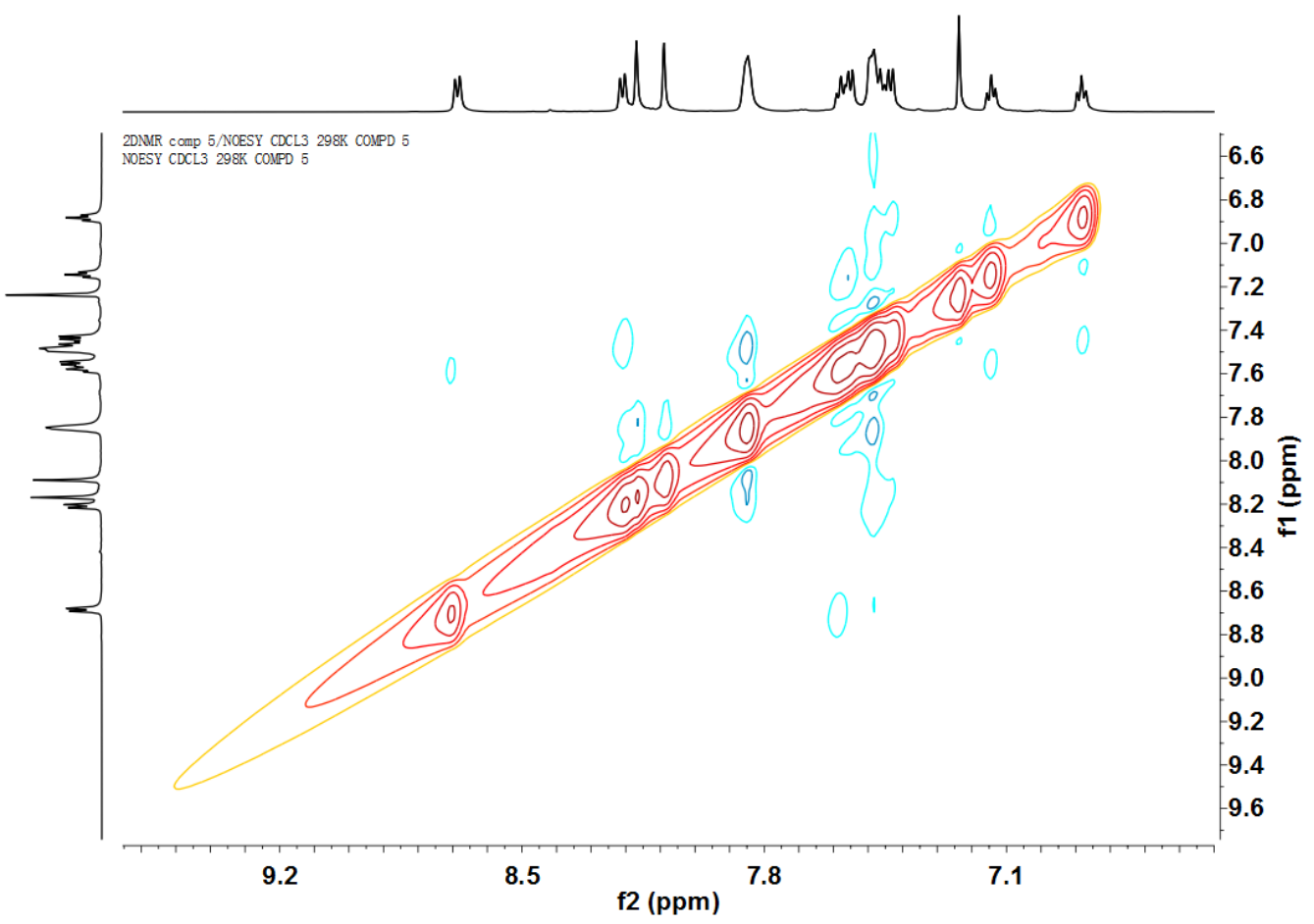

NOESY spectrum of compound $4\left(600 \mathrm{MHz}, \mathrm{CDCl}_{3}, 298 \mathrm{~K}\right)$

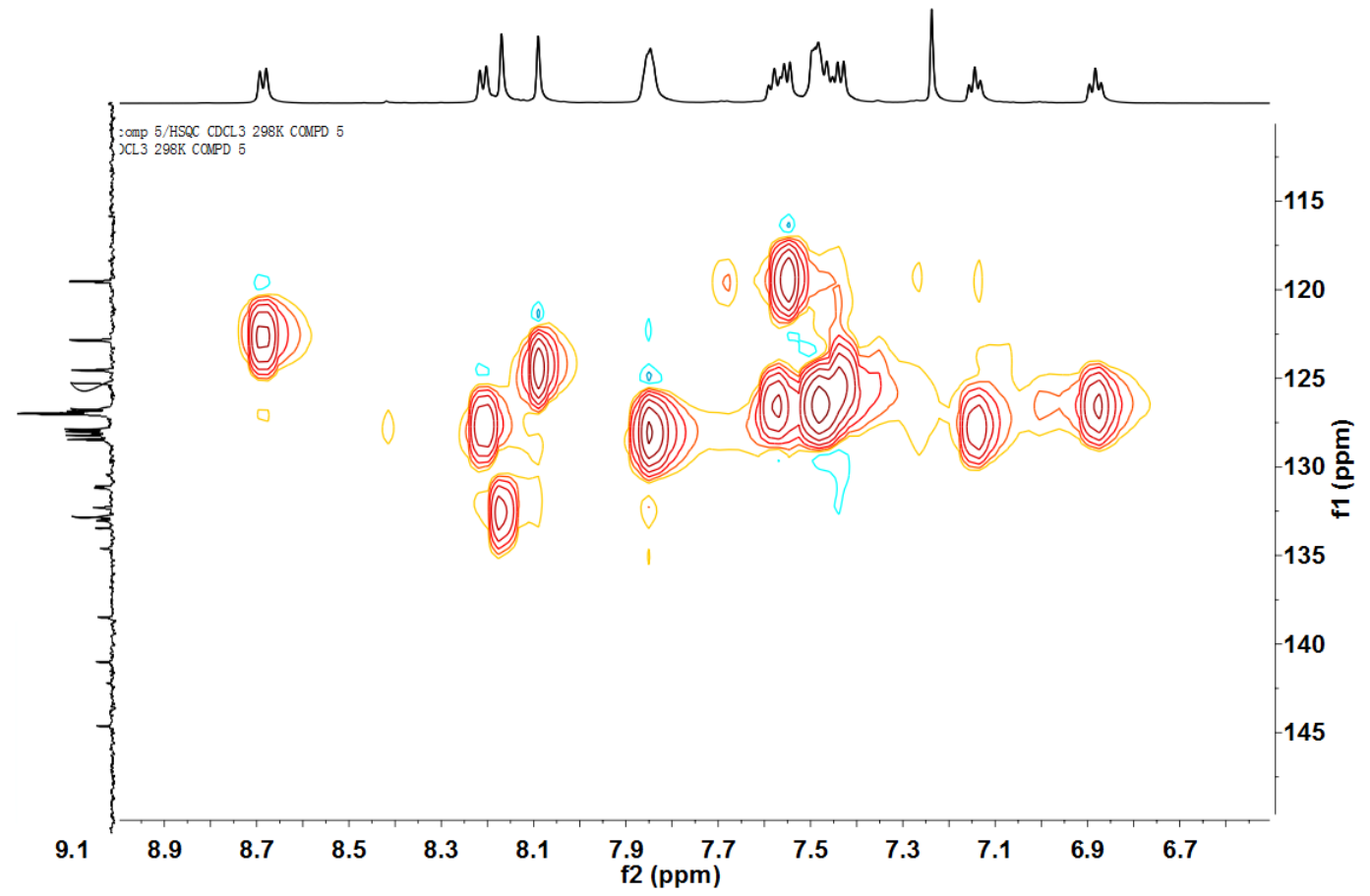

NOESY spectrum of compound $4\left(600 \mathrm{MHz}, \mathrm{CDCl}_{3}, 298 \mathrm{~K}\right)$ 


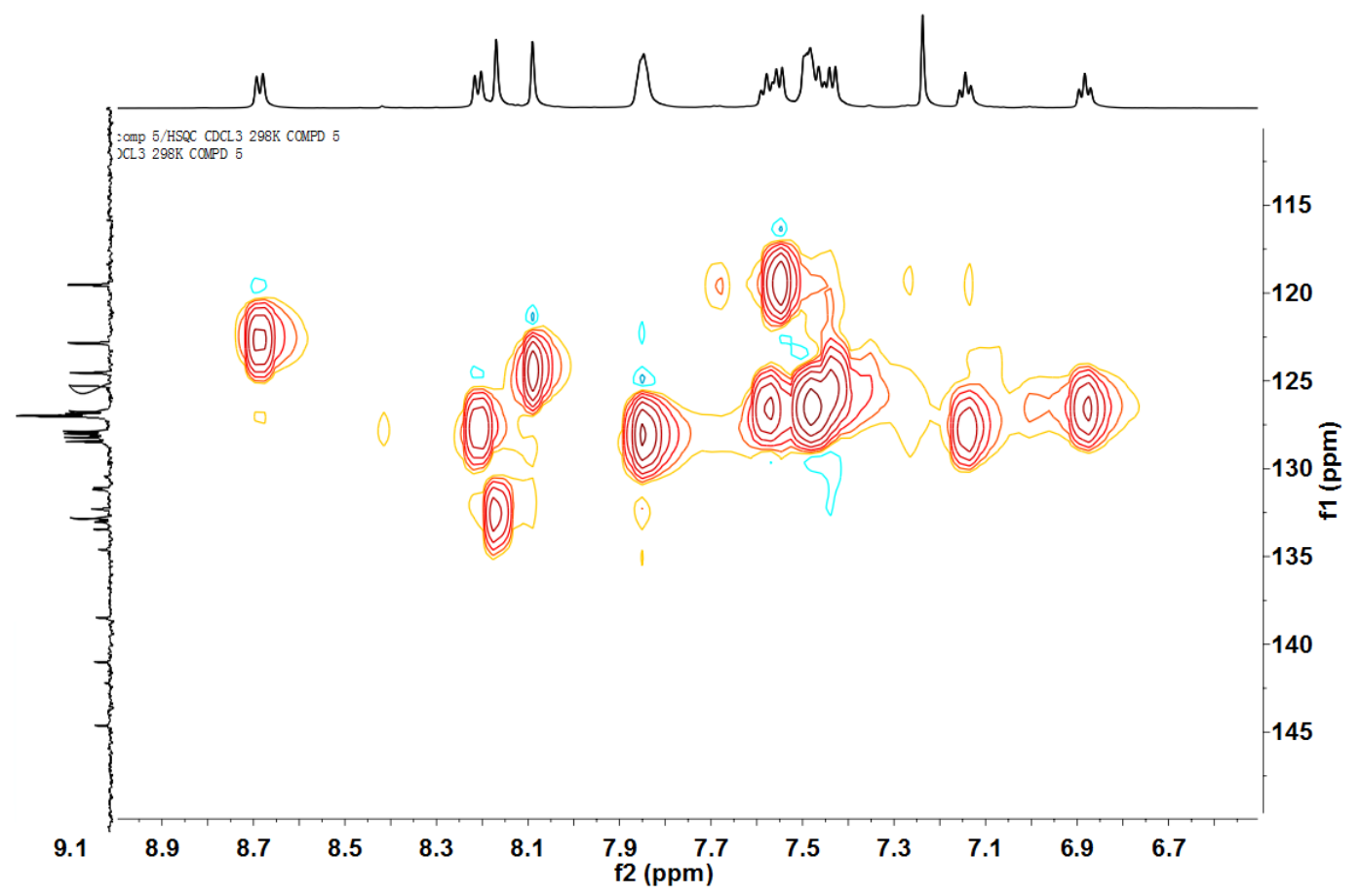

HSQC spectrum of compound $4\left(600 \mathrm{MHz}, \mathrm{CDCl}_{3}, 298 \mathrm{~K}\right)$

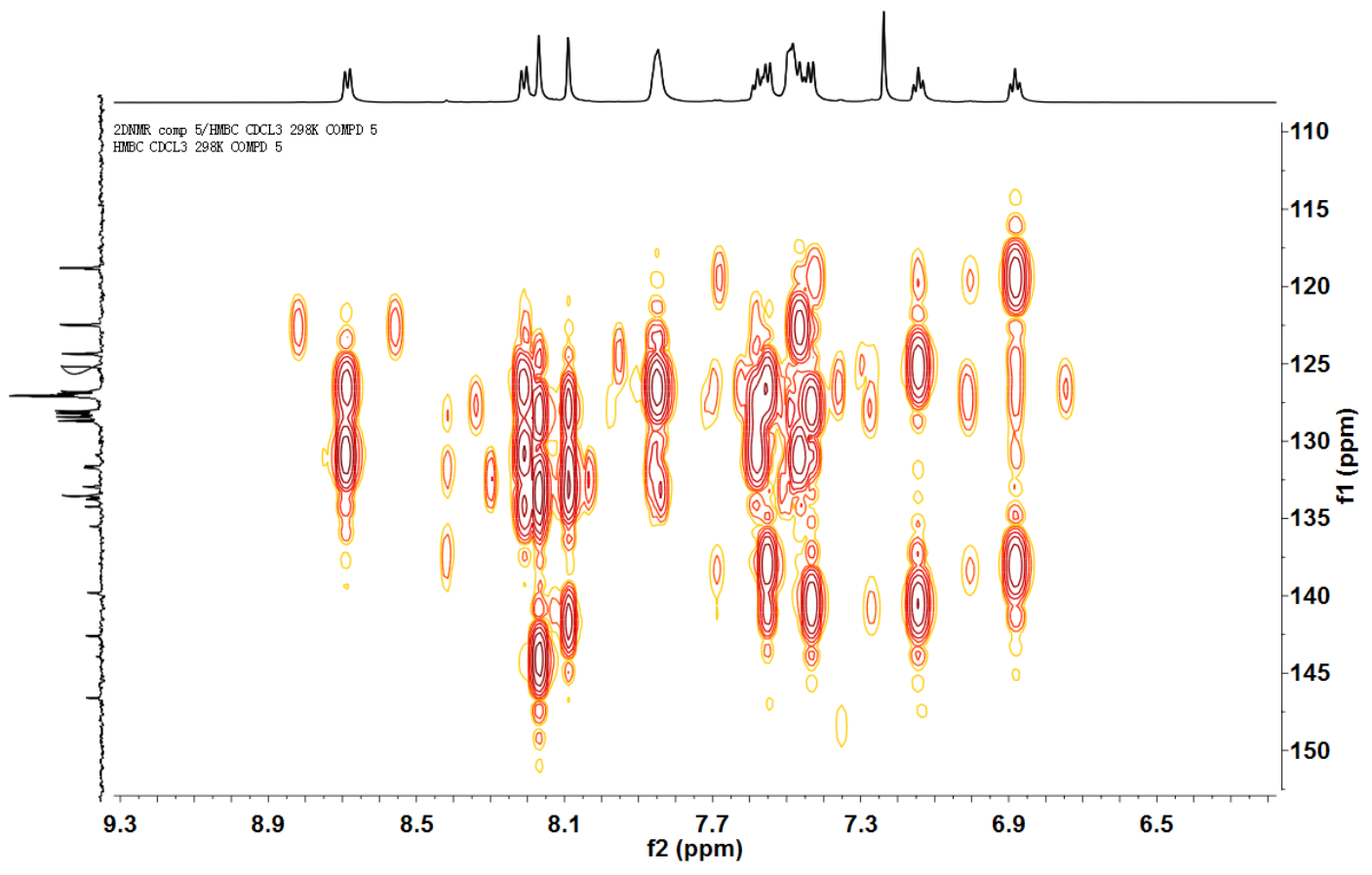

HMBC spectrum of compound $4\left(600 \mathrm{MHz}, \mathrm{CDCl}_{3}, 298 \mathrm{~K}\right)$ 


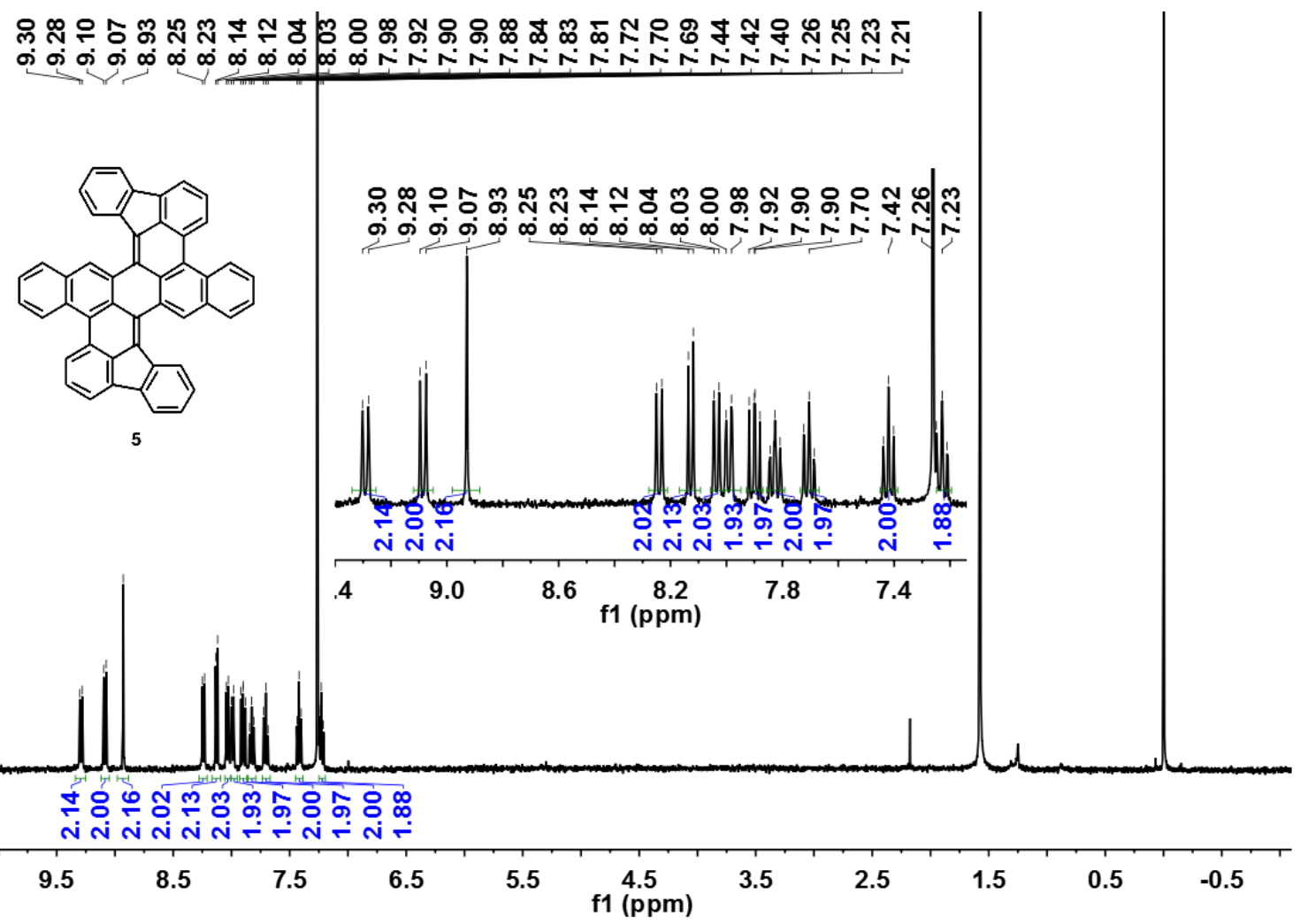

${ }^{1} \mathrm{H}$ NMR spectrum of compound $5\left(400 \mathrm{MHz} \mathrm{CDCl}_{3}, 300 \mathrm{~K}\right)$
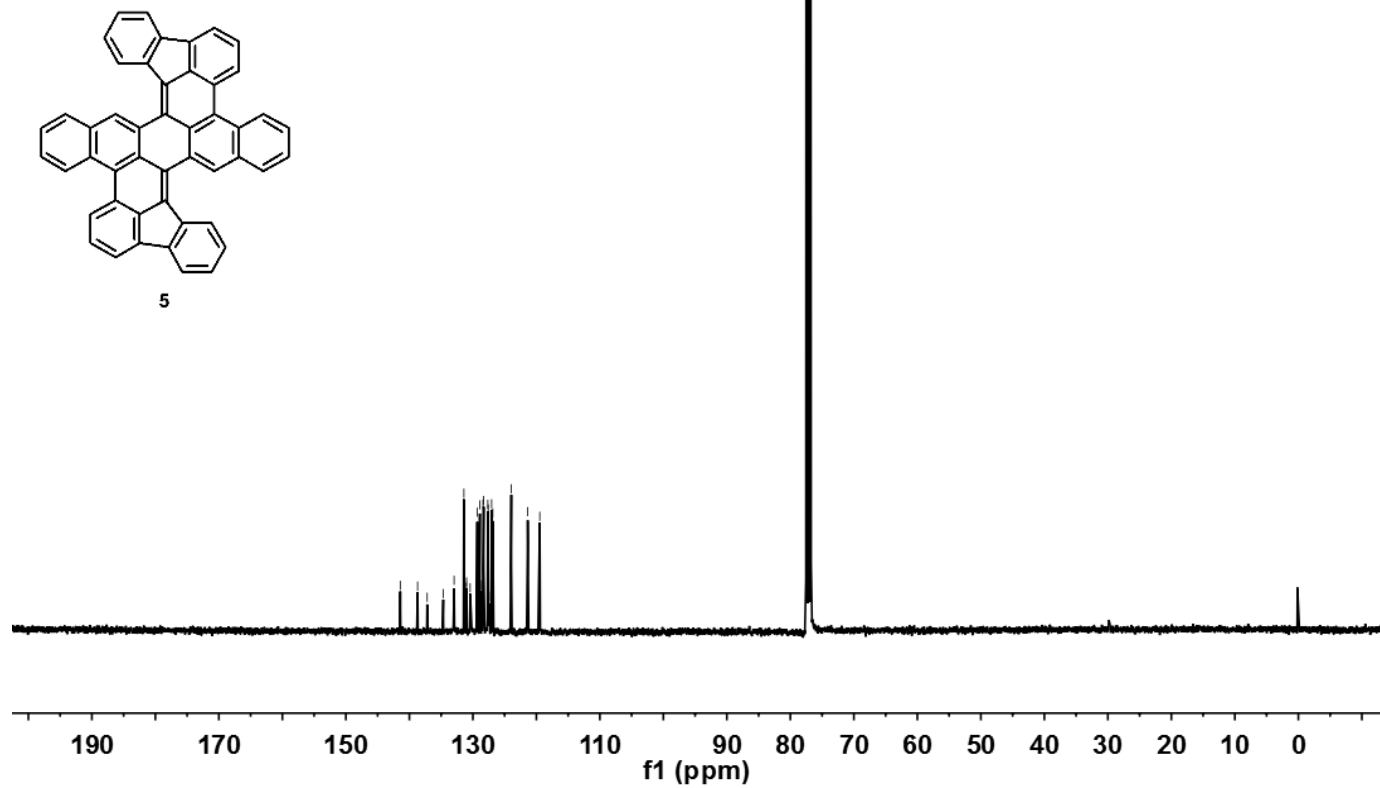

${ }^{13} \mathrm{C}$ NMR spectrum of compound 5 (101 $\left.\mathrm{MHz}, \mathrm{CDCl}_{3}, 300 \mathrm{~K}\right)$ 


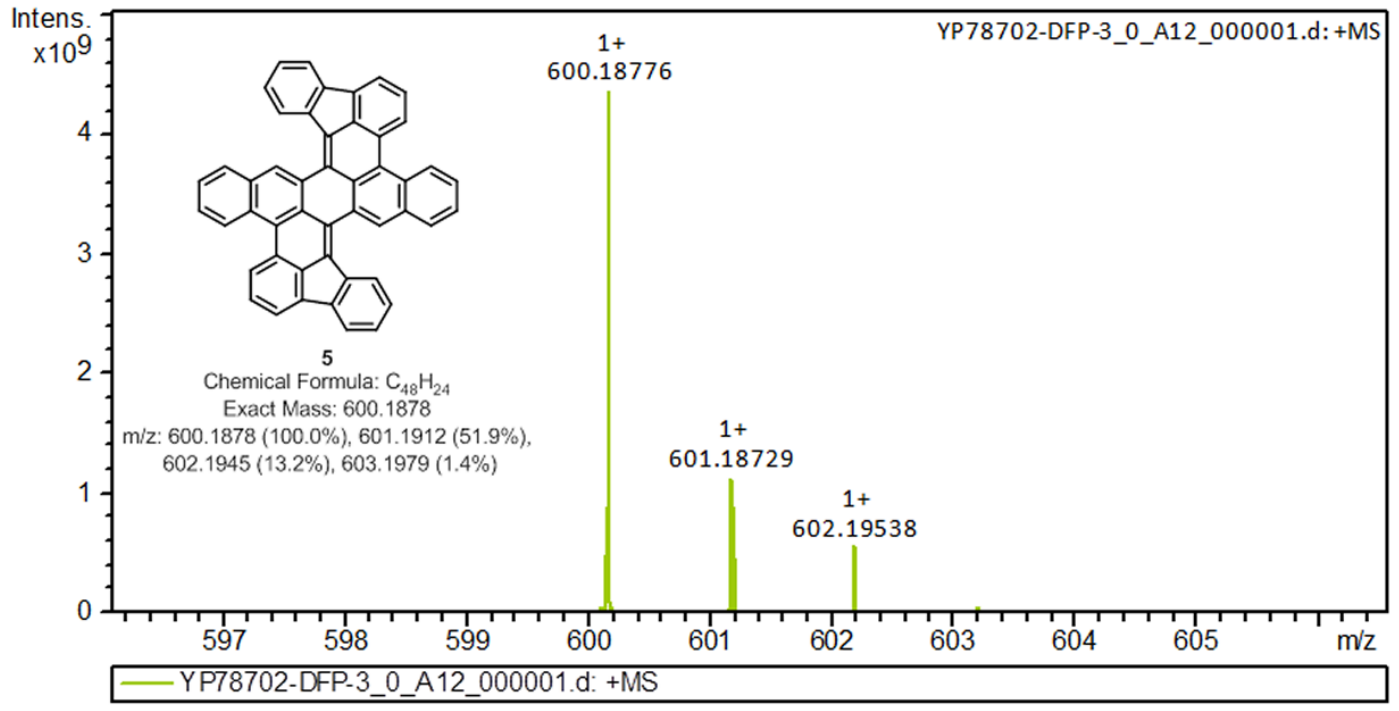

MALDI-MS of compound 5

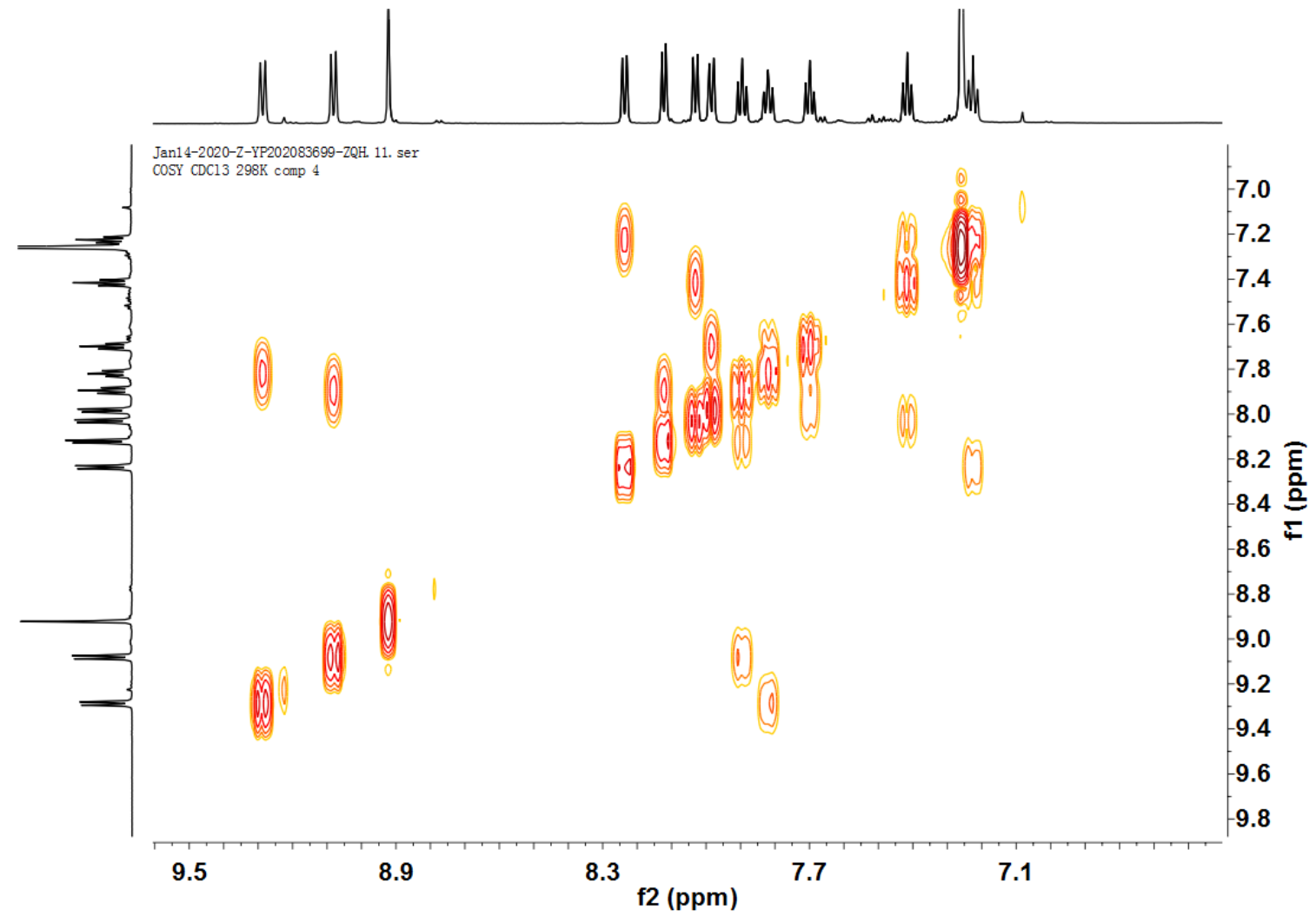

COSY spectrum of compound 5 (600 $\left.\mathrm{MHz}, \mathrm{CDCl}_{3}, 298 \mathrm{~K}\right)$ 


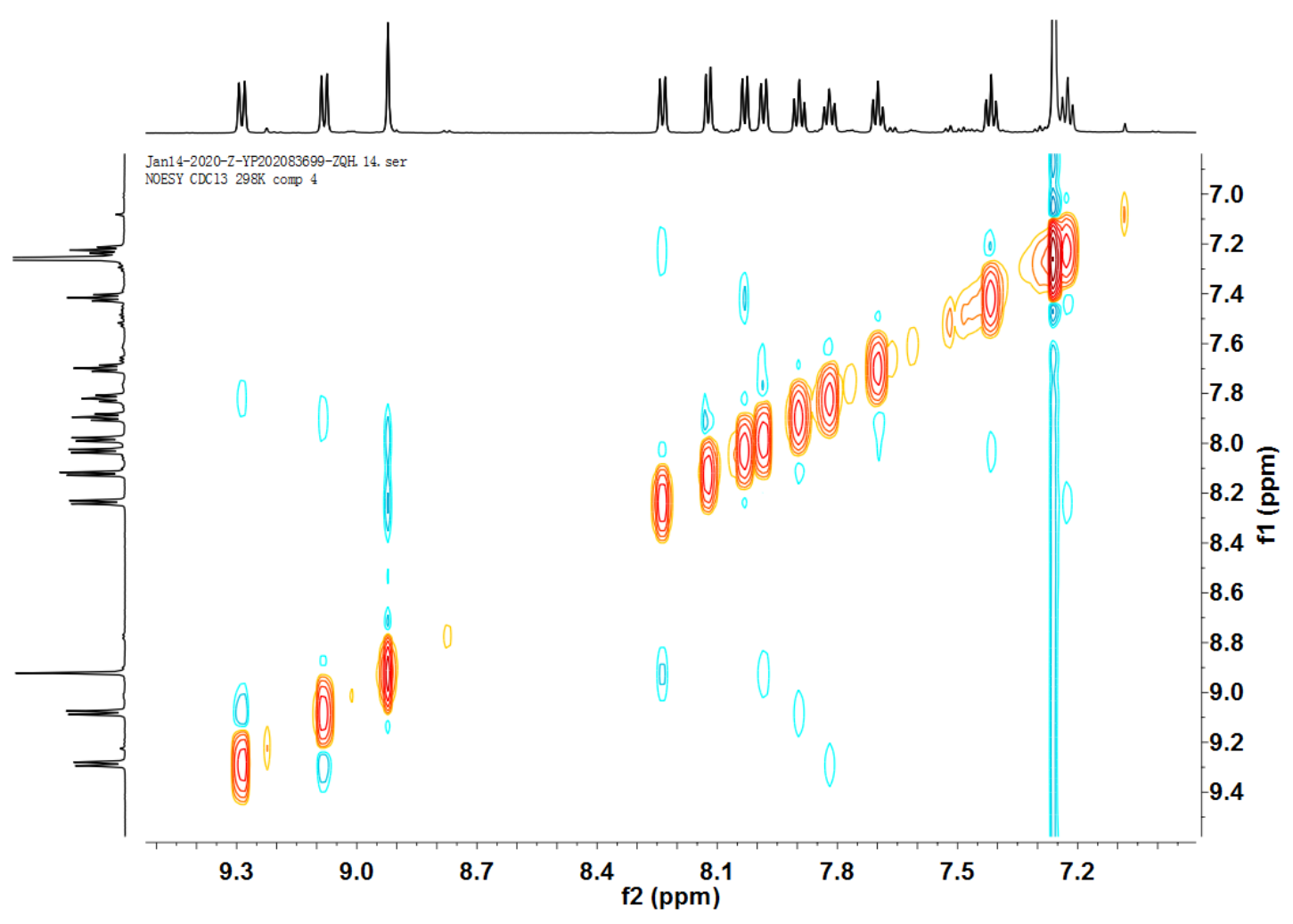

NOESY spectrum of compound $5\left(600 \mathrm{MHz}^{\left.-\mathrm{CDCl}_{3}, 298 \mathrm{~K}\right)}\right.$

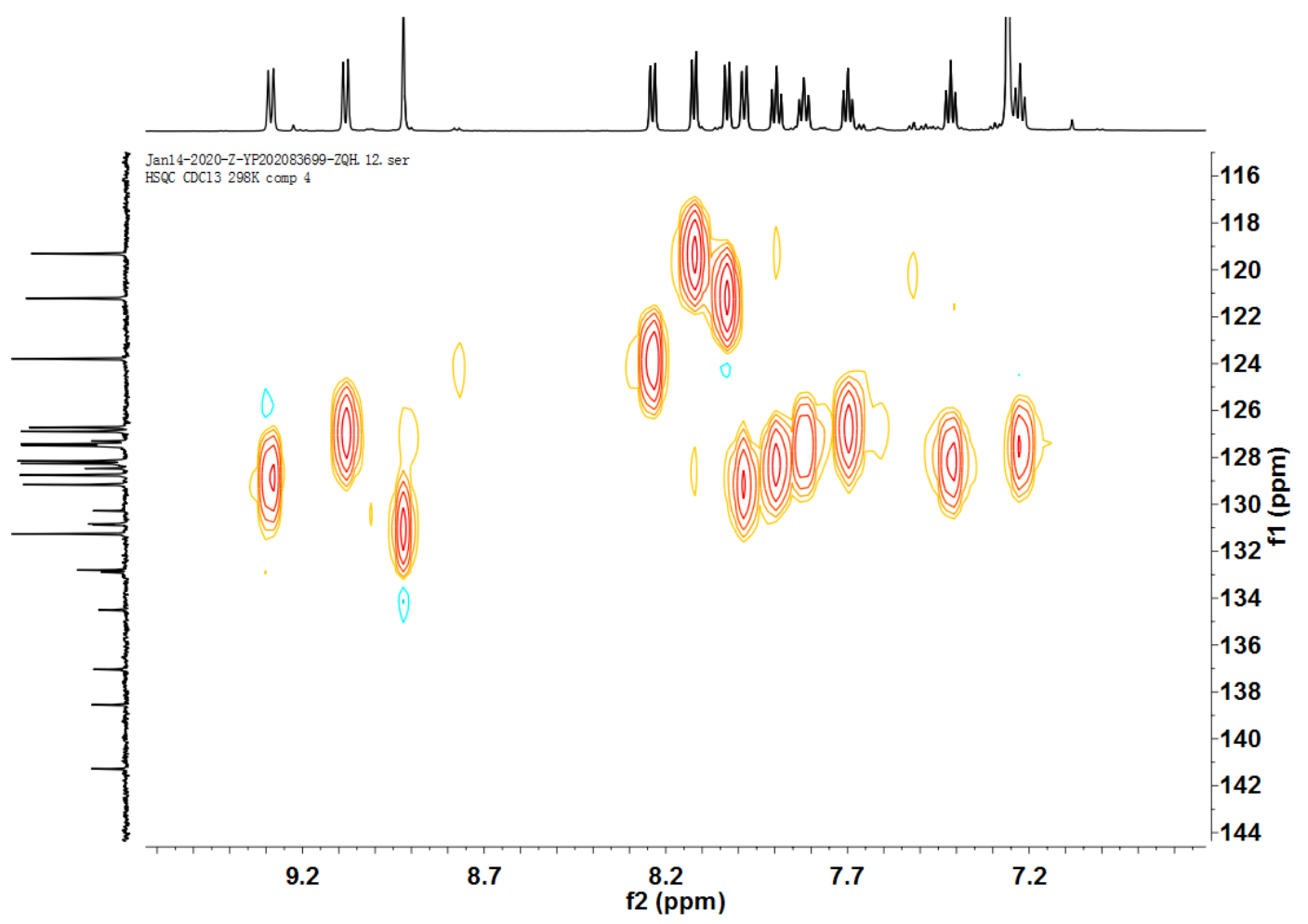

HSQC spectrum of compound 5 (600 $\left.\mathrm{MHz} \mathrm{CDCl}_{3}, 298 \mathrm{~K}\right)$ 


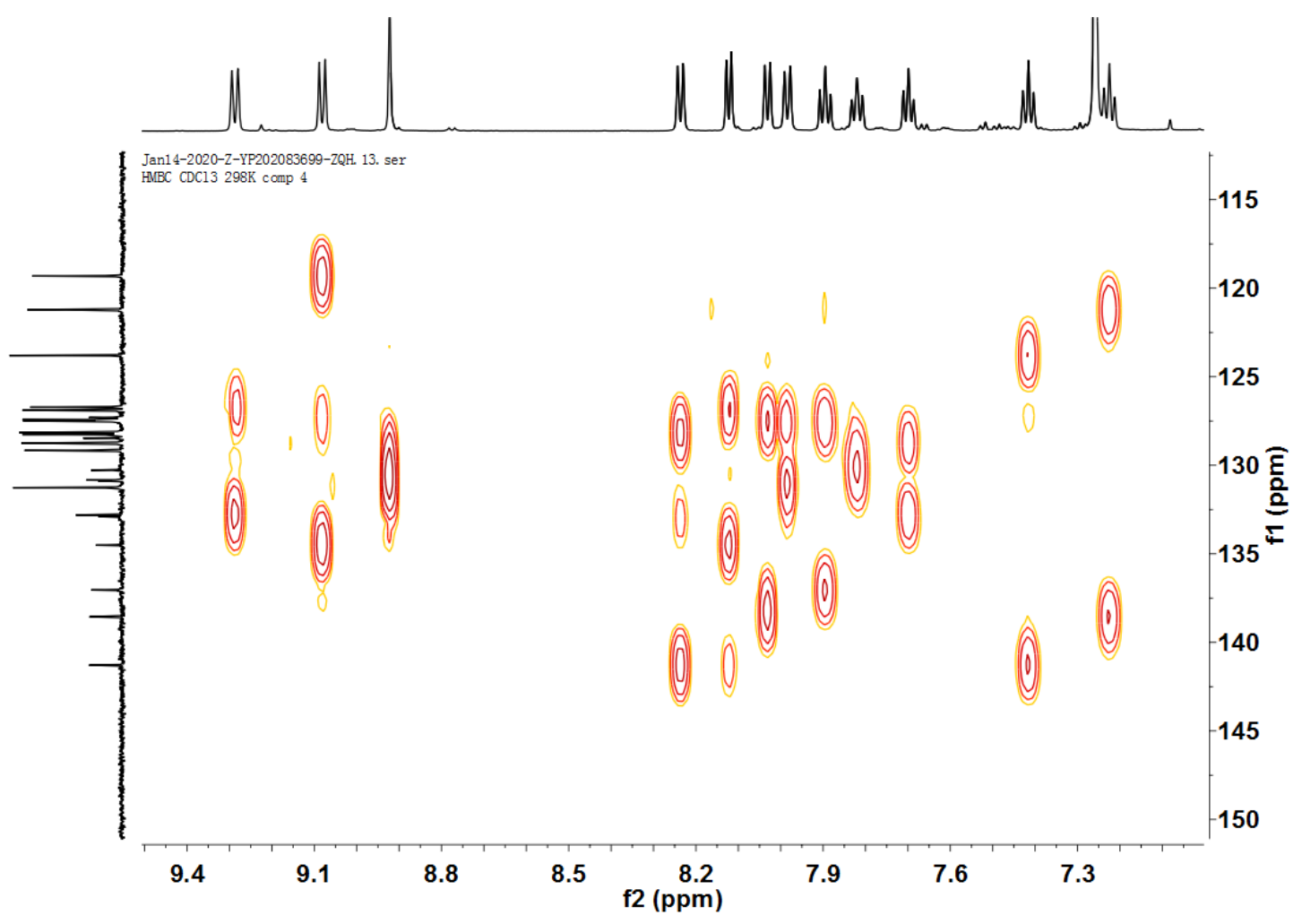

HMBC spectrum of compound 5 (600 $\left.\mathrm{MHz}, \mathrm{CDCl}_{3}, 298 \mathrm{~K}\right)$

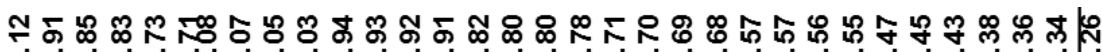

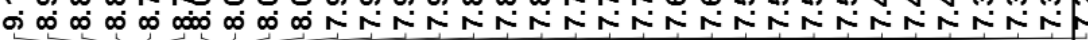

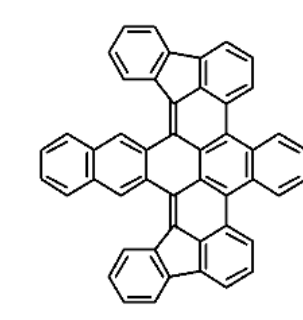

둥

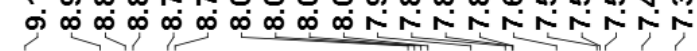
i
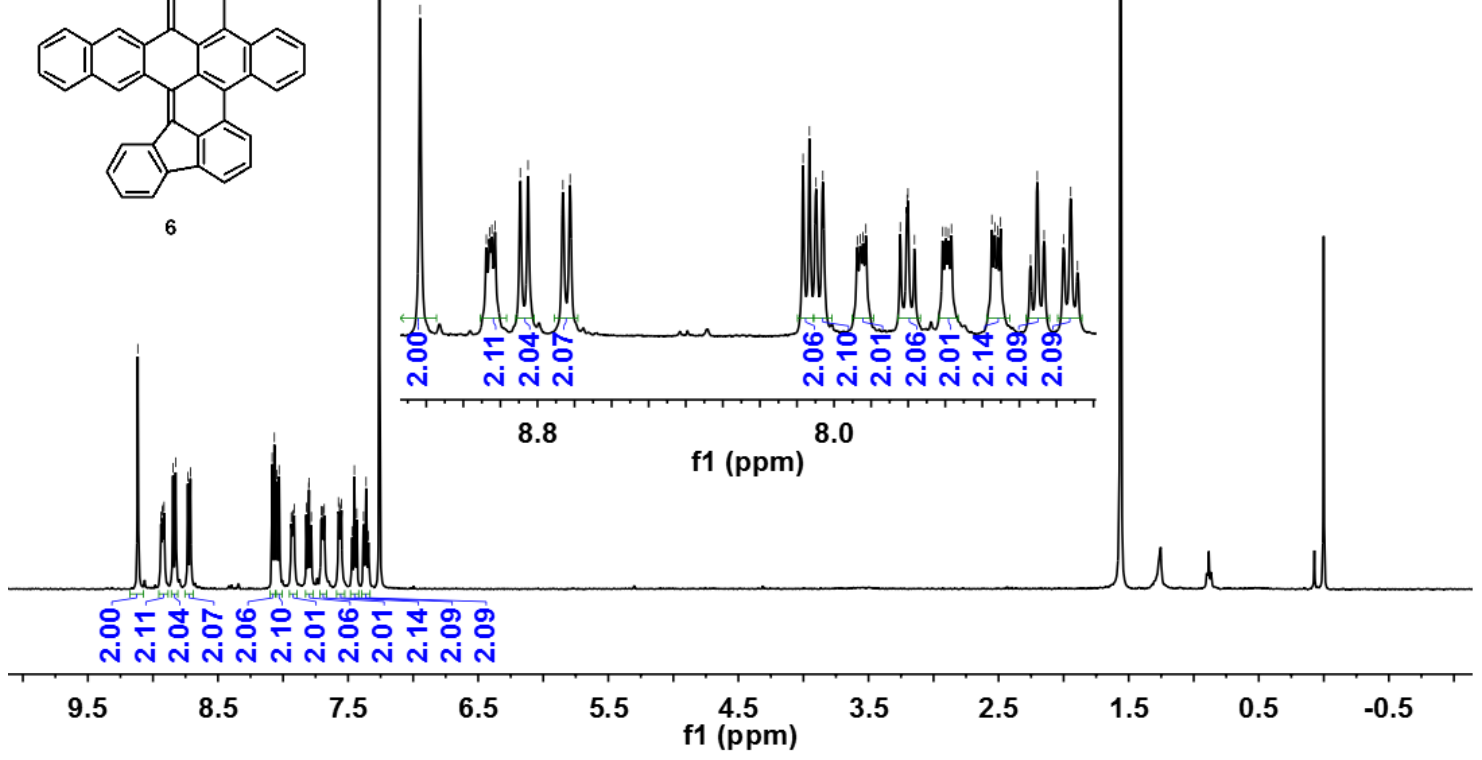

${ }^{1} \mathrm{H}$ NMR spectrum of compound $6\left(400 \mathrm{MHz} \mathrm{CDCl}_{3}, 300 \mathrm{~K}\right)$ 

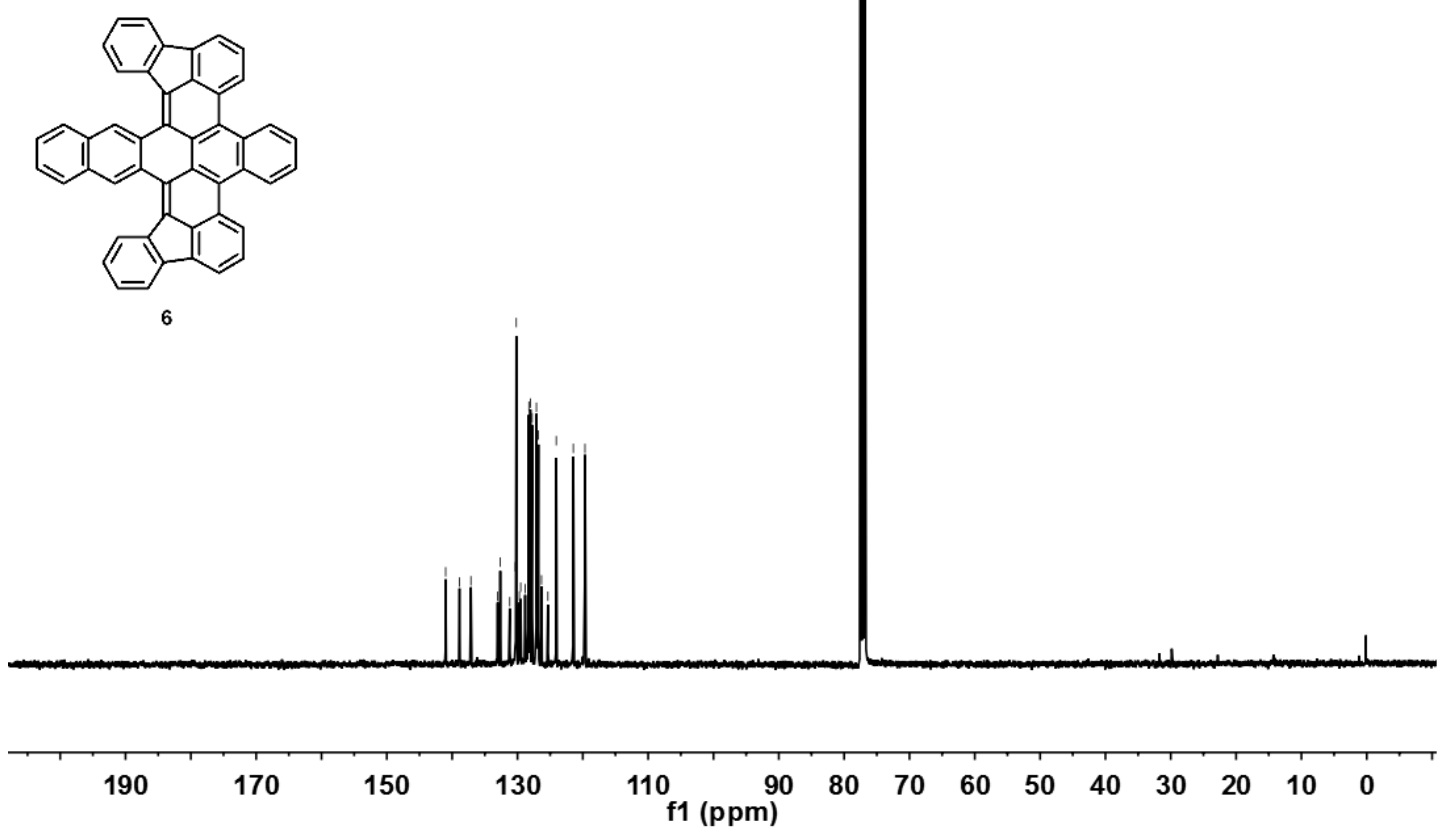

${ }^{13} \mathrm{C}$ NMR spectrum of compound 6 (101 $\left.\mathrm{MHz}, \mathrm{CDCl}_{3}, 300 \mathrm{~K}\right)$

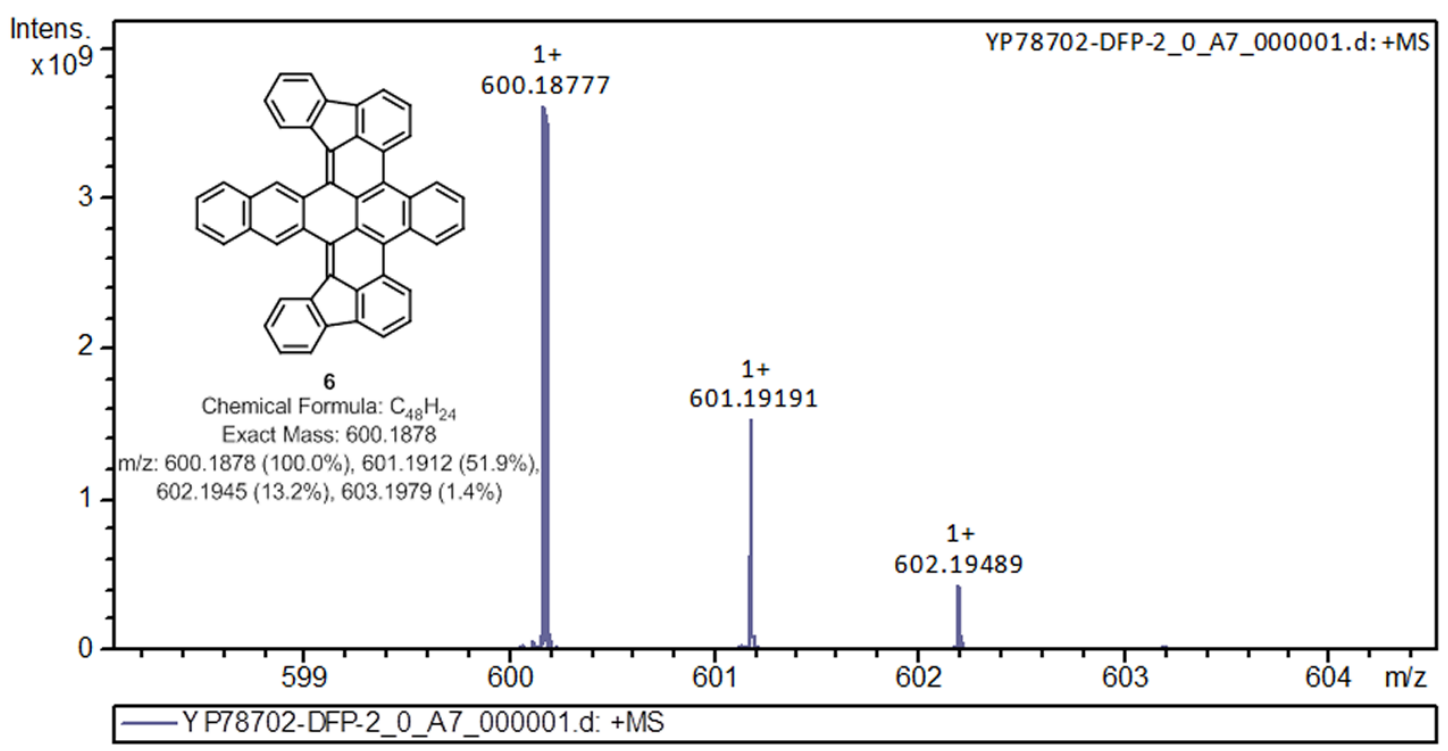

MALDI-MS of compound 6 


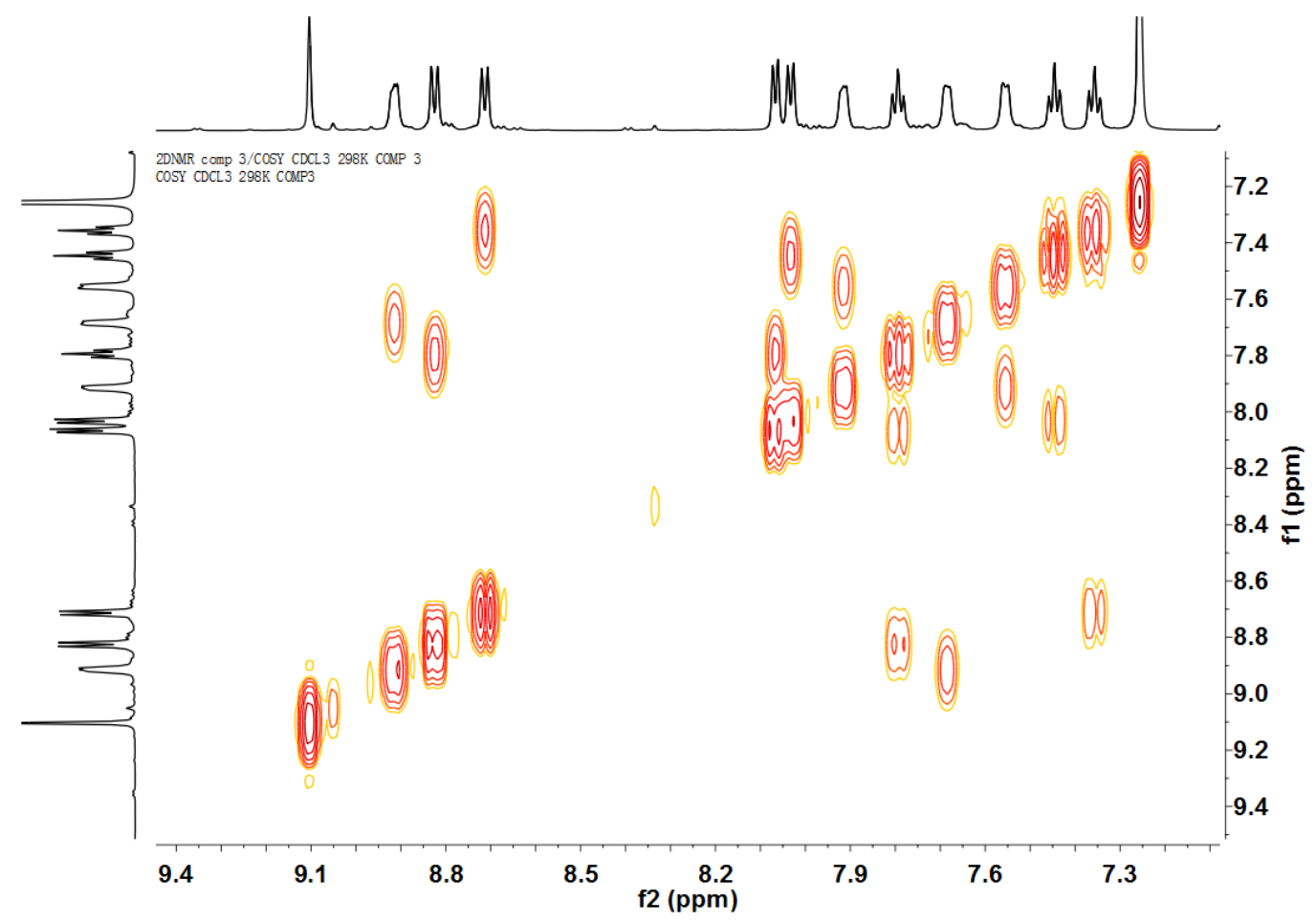

COSY spectrum of compound $6\left(600 \mathrm{MHz}, \mathrm{CDCl}_{3}, 298 \mathrm{~K}\right)$

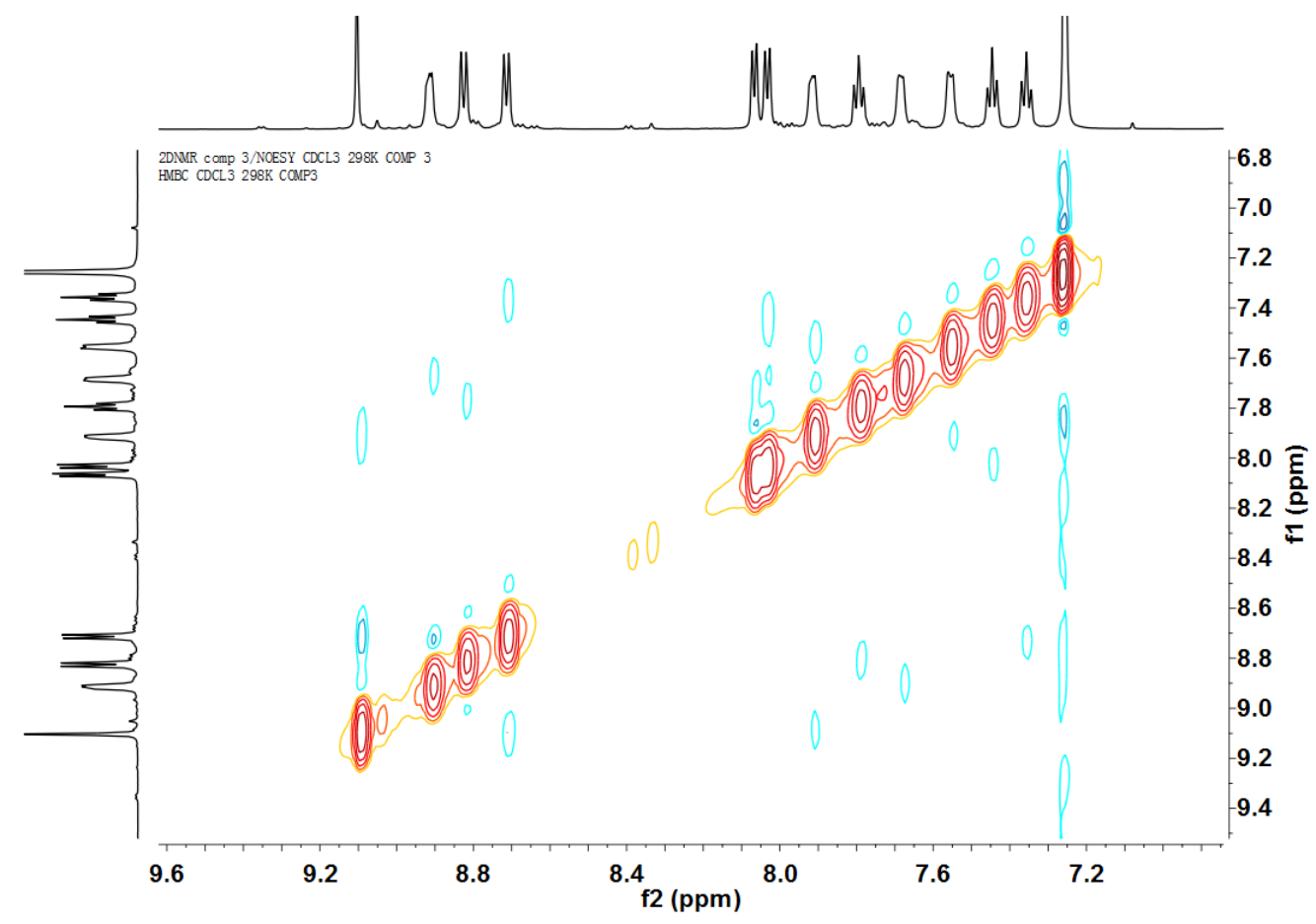

NOESY spectrum of compound $6\left(600 \mathrm{MHz}, \mathrm{CDCl}_{3}, 298 \mathrm{~K}\right)$ 


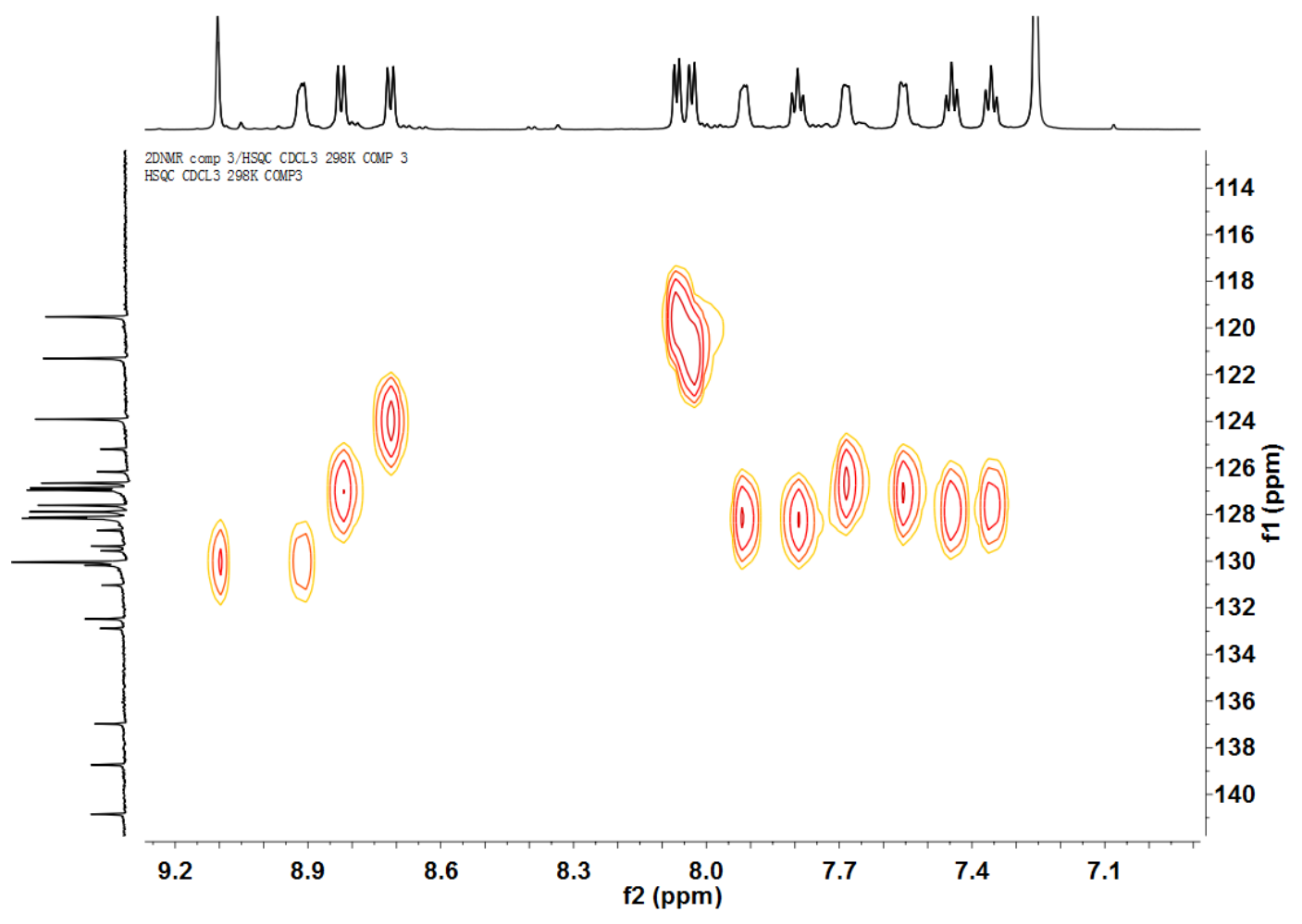

HSQC spectrum of compound $6\left(600 \mathrm{MHz}, \mathrm{CDCl}_{3}, 298 \mathrm{~K}\right)$

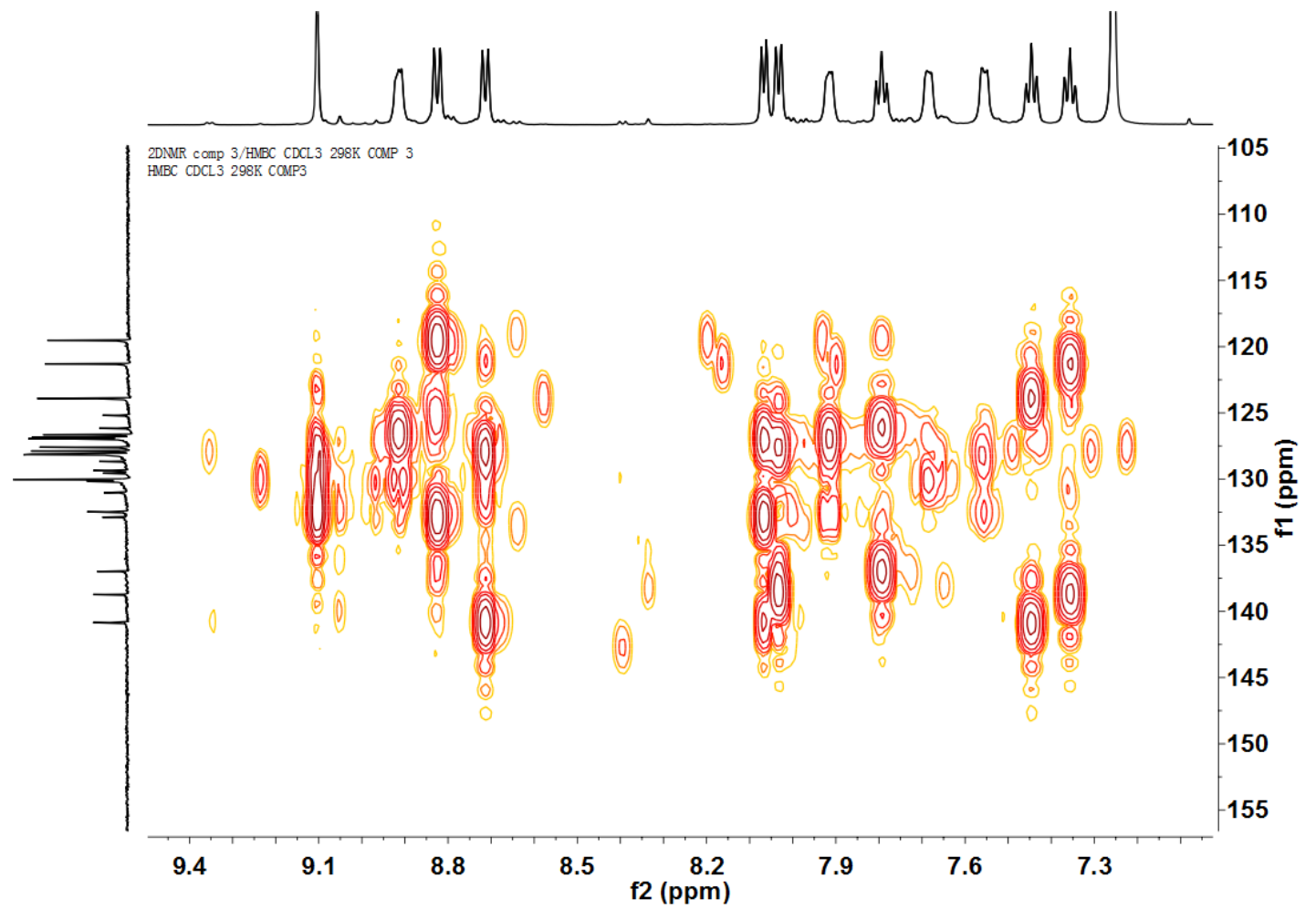

HMBC spectrum of compound $6\left(600 \mathrm{MHz}, \mathrm{CDCl}_{3}, 298 \mathrm{~K}\right)$ 


\section{Crystal information of compounds 2-6 (Figure S2-S6, Table S2-S6)}

a)

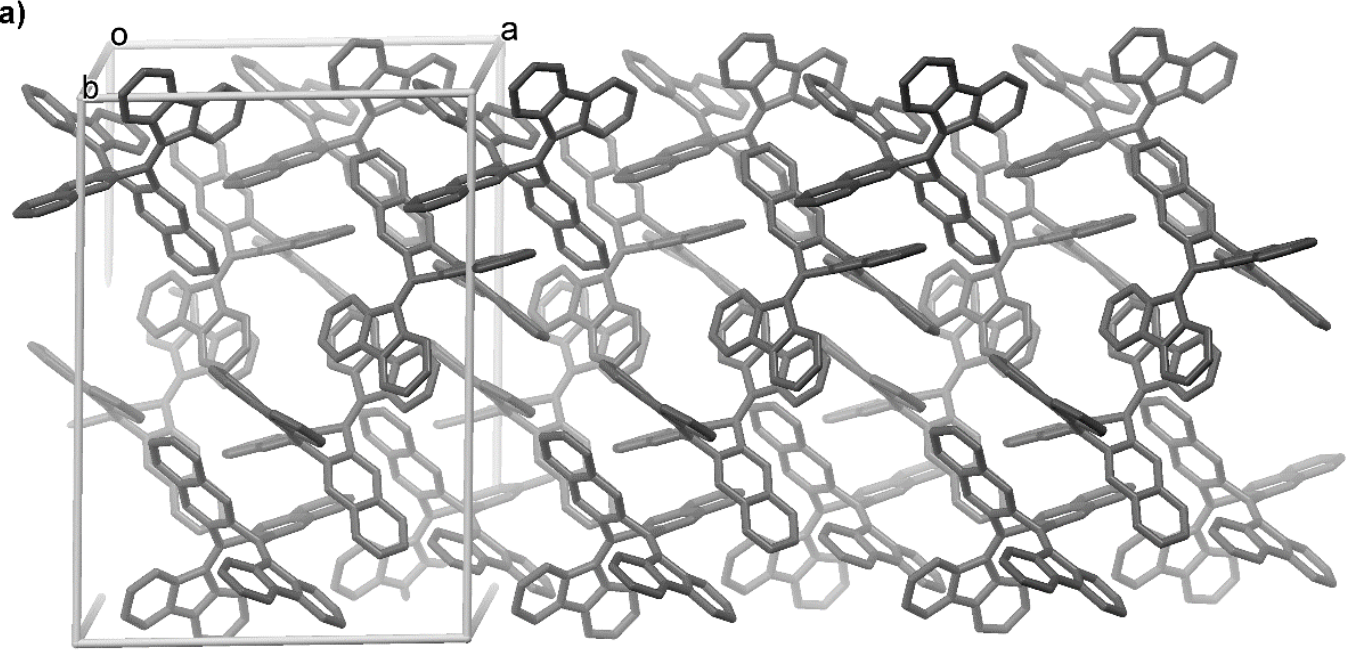

b)
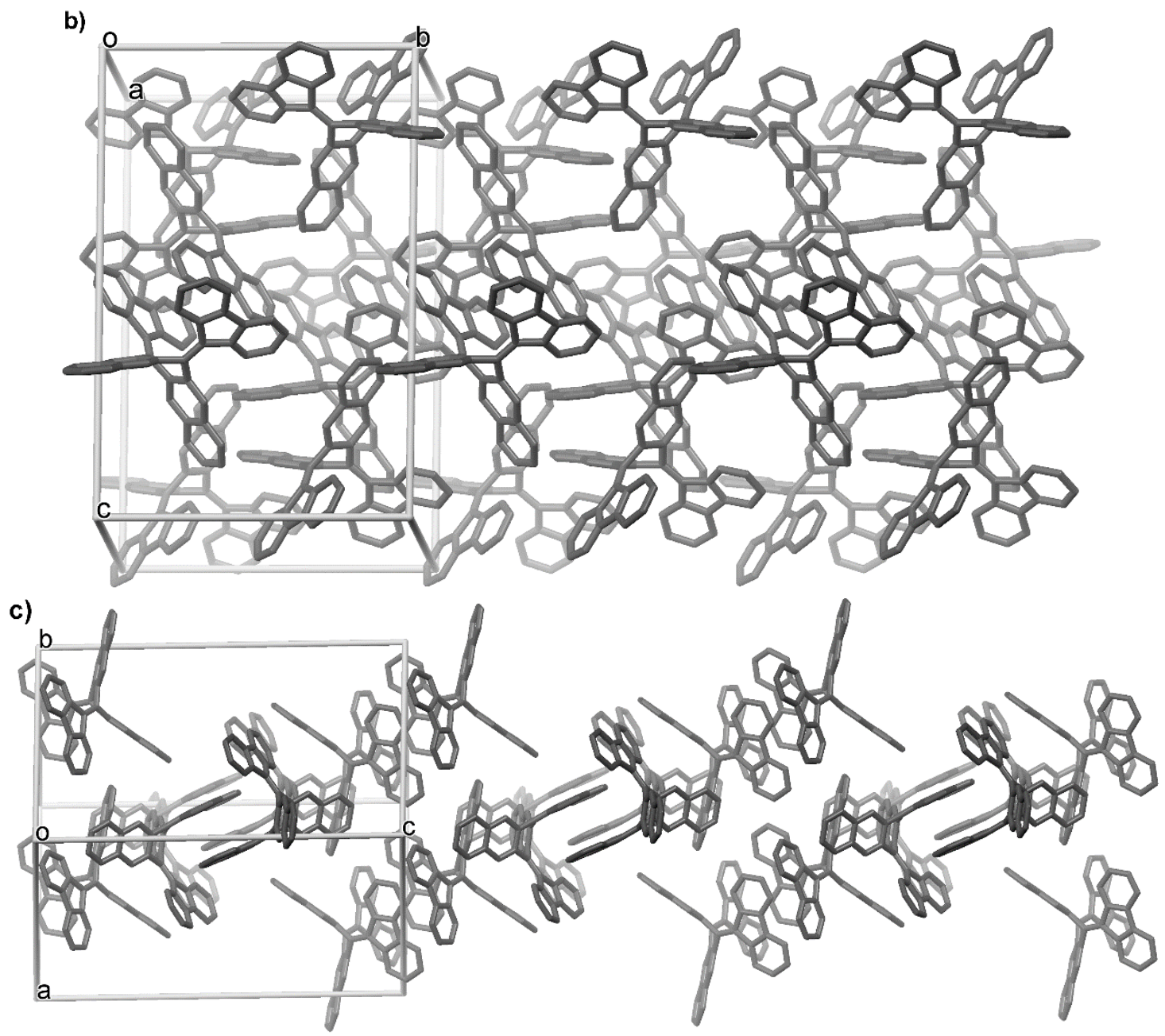

Figure S2. Stacking of compound $\mathbf{2}$ in single crystal with depth cueing view: a) along a axis, b) along b axis, c) along c axis (hydrogen atoms are omitted for clarity). Crystal of $\mathbf{2}$ was grew by diffusing methonal into solution of $\mathbf{2}$ in chloroform. 
a)

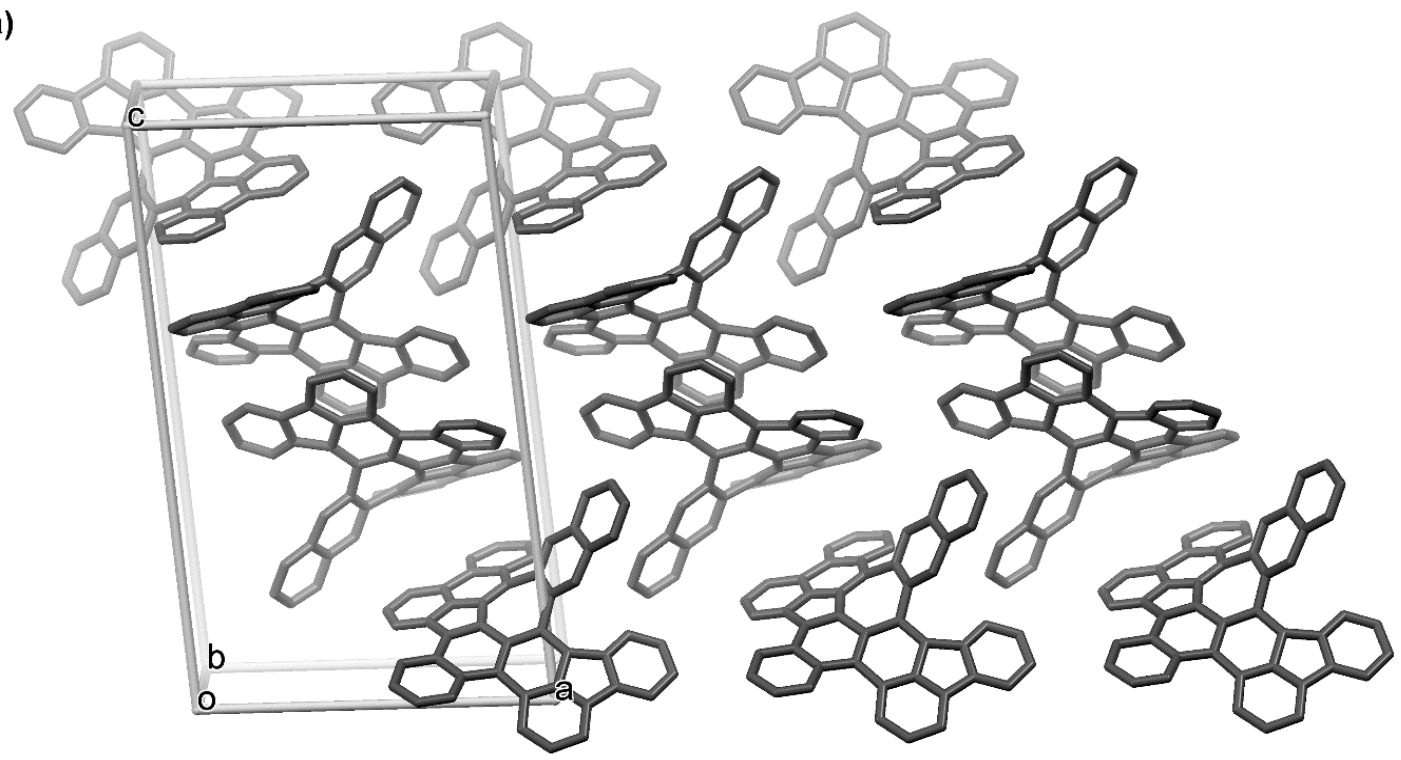

b)
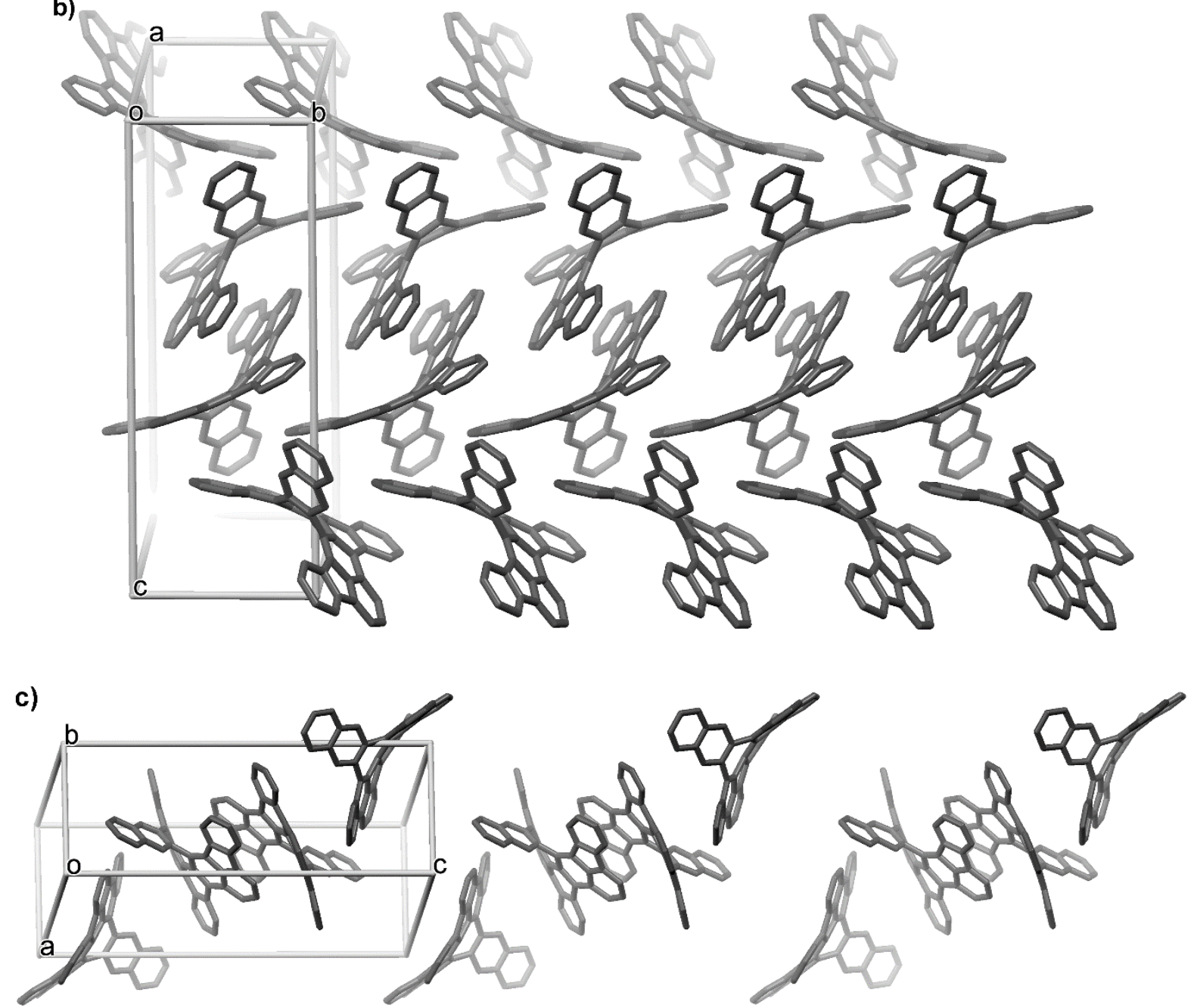

Figure S3. Stacking of compound $\mathbf{3}$ in single crystal with depth cueing view: a) along a axis, b) along b axis, c) along c axis (hydrogen atoms are omitted for clarity). Crystal of $\mathbf{3}$ was grew by diffusing methonal into solution of $\mathbf{3}$ in 1,2-dichloroethane. 
a)
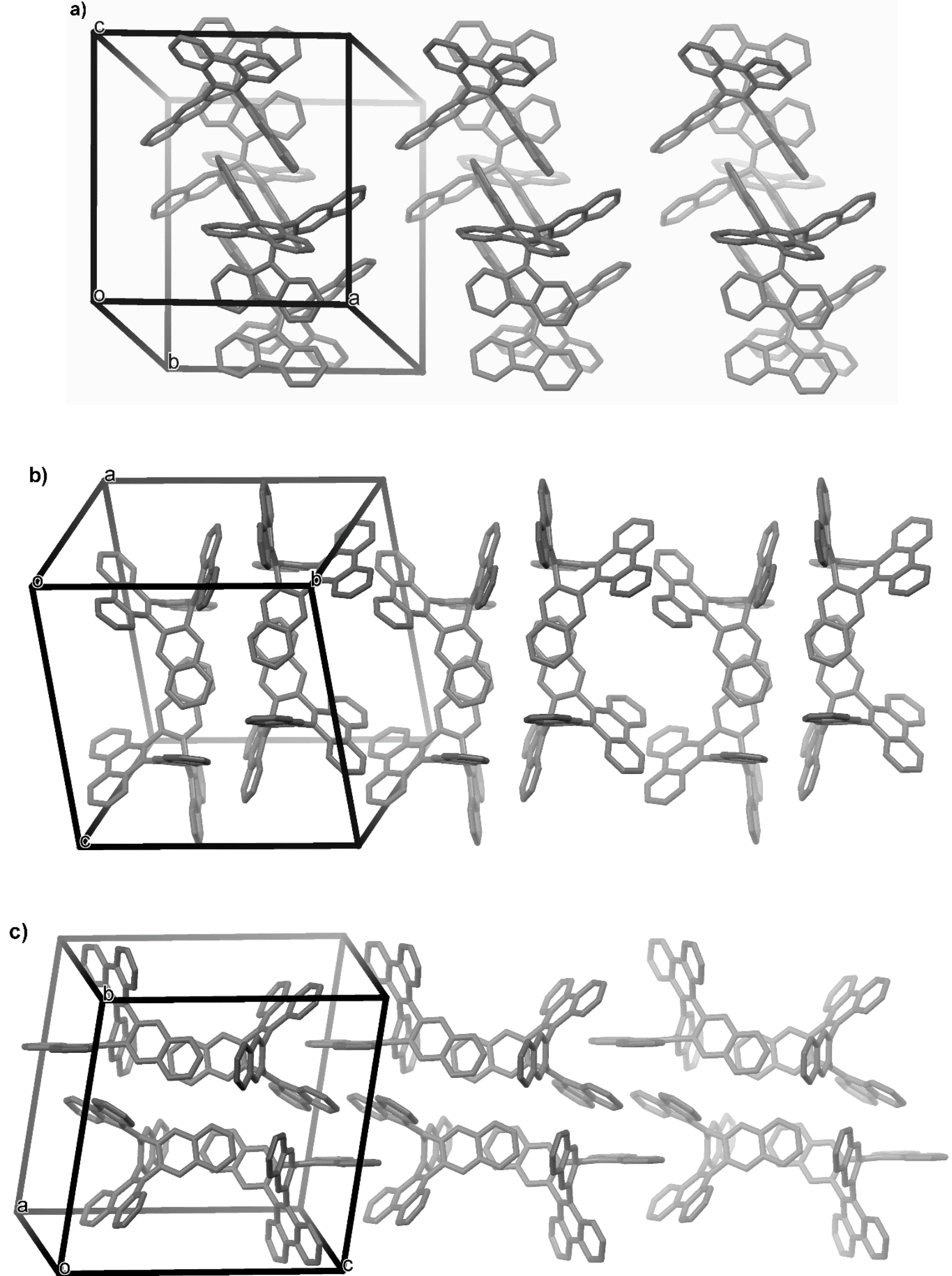

Figure S4. Stacking of compound $\mathbf{4}$ in single crystal with depth cueing view: a) along a axis, b) along b axis, c) along c axis (hydrogen atoms are omitted for clarity). Crystal of $\mathbf{4}$ was grew by diffusing methonal into solution of $\mathbf{4}$ in cyclohexane and chloroform. 
a)

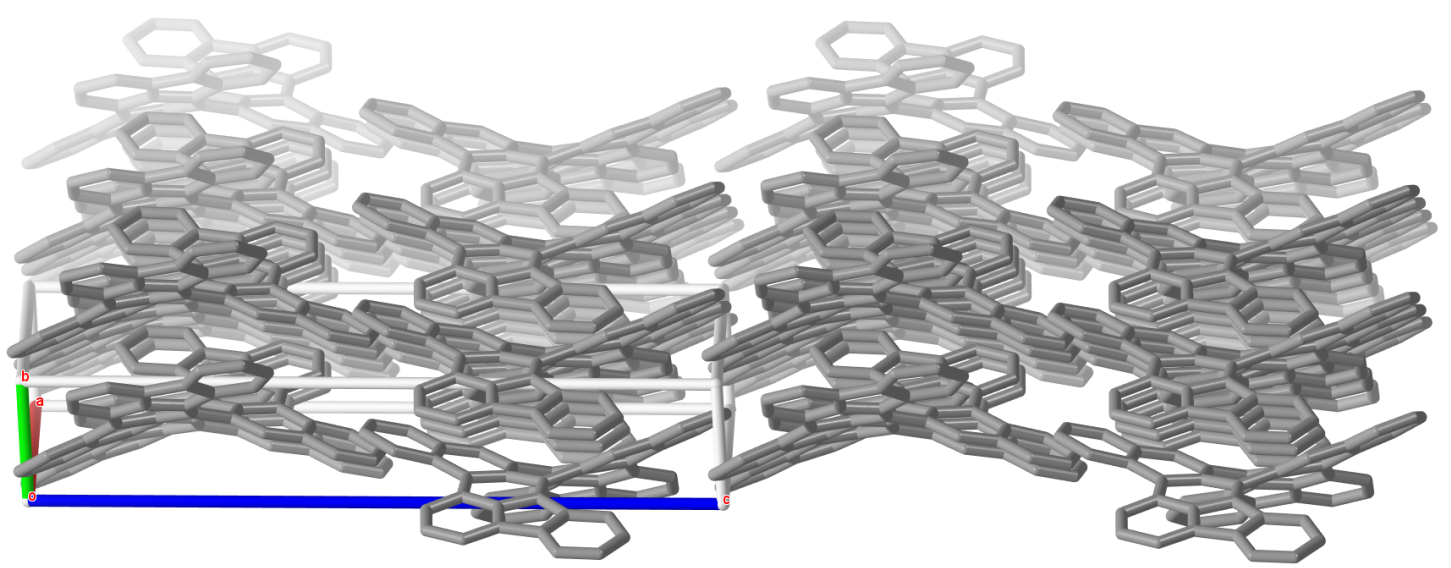

b)

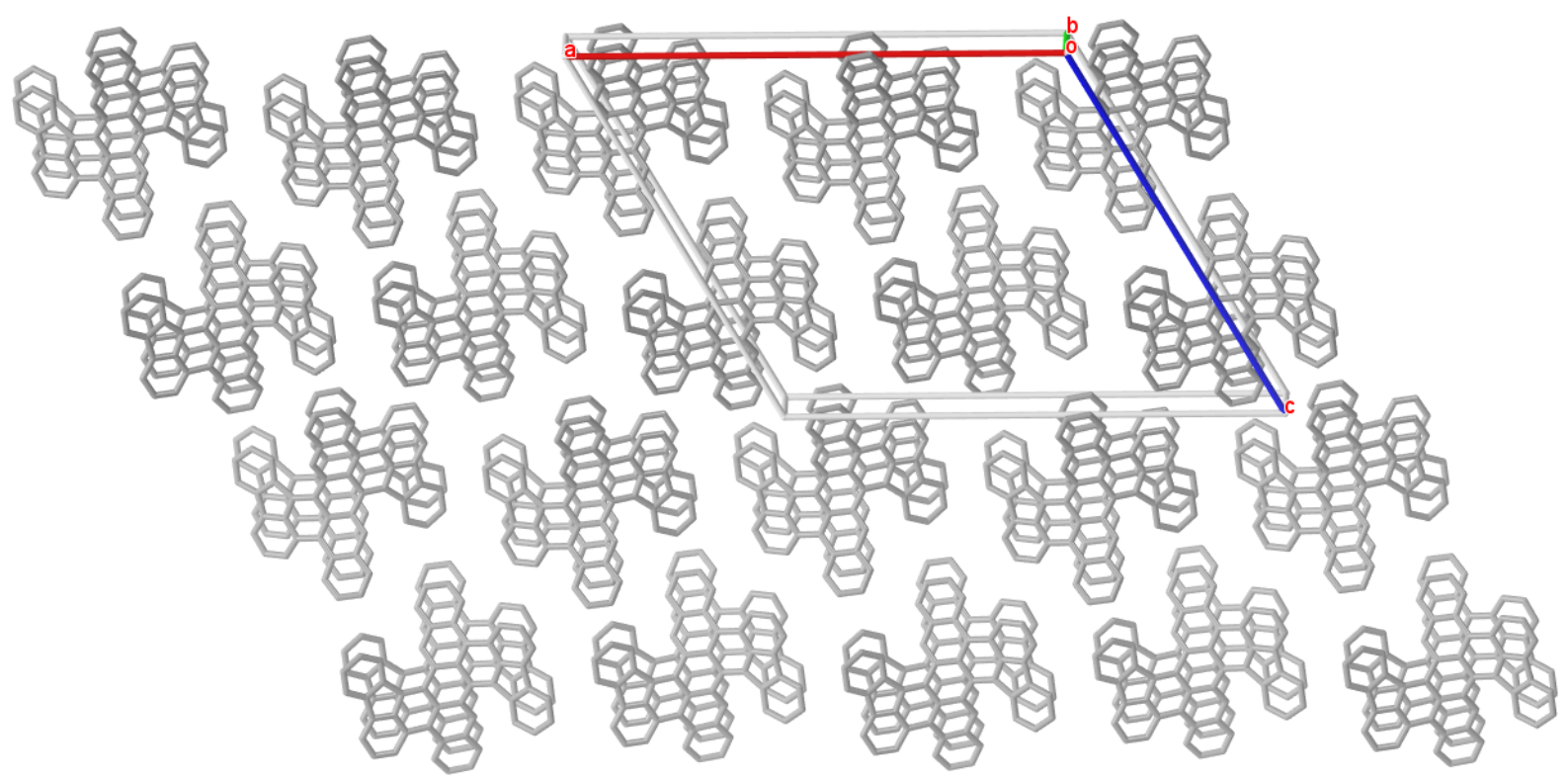

c)
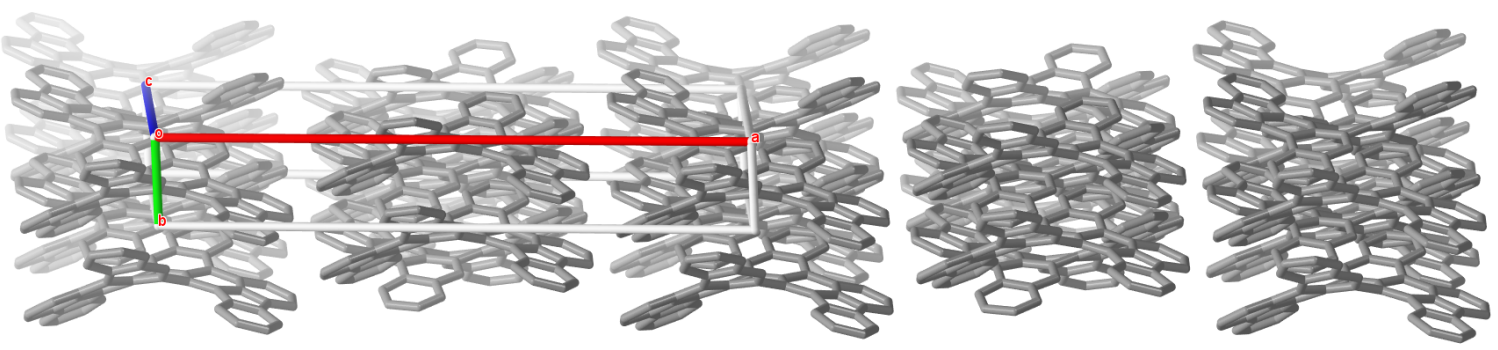

Figure S5. Stacking of compound 4 in single crystal with depth cueing view: a) along a axis, b) along b axis, c) along c axis (hydrogen atoms are omitted for clarity). Crystal of $\mathbf{4}$ was grew by diffusing of methonal into solution of $\mathbf{4}$ in cyclohexane and chloroform. 
a)
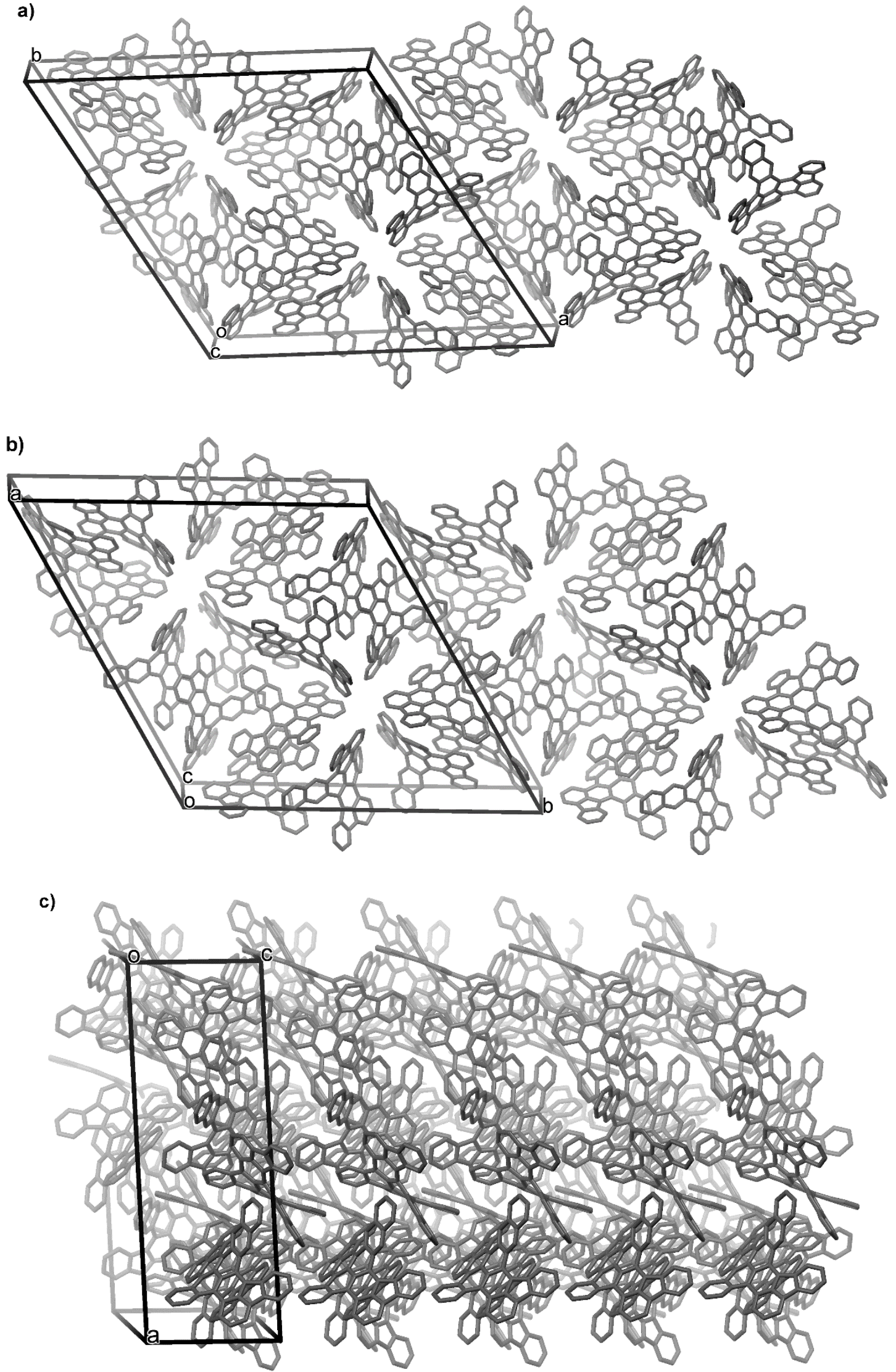

Figure S6. Stacking of compound $\mathbf{6}$ in single crystal with depth cueing view: a) along a axis, b) along b axis, c) along c axis (Hydrogen atoms are omitted for clarity). Crystal of $\mathbf{6}$ was grew by diffusing of methonal into solution of $\mathbf{6}$ in chloroform. 
Table S2 Crystal data and structure determination for $\mathbf{2}$

\begin{tabular}{|c|c|}
\hline Compound & 2 \\
\hline Chemical formula & $\mathrm{C}_{48} \mathrm{H}_{28}$ \\
\hline Formula mass & 604.70 \\
\hline Crystal system & Monoclinic \\
\hline Space group & $\mathrm{C} 2 / \mathrm{c}$ \\
\hline$\lambda(\AA)$ & 1.54178 \\
\hline$a, b, c(\AA)$ & $17.4578(17), 15.9765(19), 24.339(3)$ \\
\hline$\alpha, \beta, \gamma\left({ }^{\circ}\right)$ & $90,92.026(9), 90$ \\
\hline$Z$ & 8 \\
\hline$V\left(\AA^{3}\right)$ & $6784.3(13)$ \\
\hline Temperature (K) & $297(2) \mathrm{K}$ \\
\hline Size $/ \mathrm{mm}^{3}$ & $0.200 \times 0.180 \times 0.160$ \\
\hline Density $\left(\mathrm{g} / \mathrm{cm}^{-3}\right)$ & 1.184 \\
\hline Measured reflections & 35680 \\
\hline Unique reflections & 6209 \\
\hline Parameters & 433 \\
\hline$R_{\mathrm{int}}$ & 0.0399 \\
\hline$\theta$ range $\left(^{\circ}\right)$ & $3.634-68.333$ \\
\hline$R_{1}, w R_{2}$ & $0.0567,0.2182$ (Final R indices) \\
\hline$S(\mathrm{GOF})$ & 1.021 \\
\hline Max/min res. dens. $\left(\mathrm{e} / \AA^{3}\right)$ & $0.359 /-0.209$ \\
\hline
\end{tabular}


Table S3 Crystal data and structure determination for $\mathbf{3}$

\begin{tabular}{|c|c|}
\hline Compound & 3 \\
\hline Chemical formula & $\mathrm{C}_{48} \mathrm{H}_{24}$ \\
\hline Formula mass & 600.67 \\
\hline Crystal system & Monoclinic \\
\hline Space group & P $121 / \mathrm{n} 1$ \\
\hline$\lambda(\AA)$ & 1.34139 \\
\hline$a, b, c(\AA)$ & $14.0068(13), 8.9759(8), 23.745(2)$ \\
\hline$\alpha, \beta, \gamma\left({ }^{\circ}\right)$ & $90^{\circ}, 94.544(6)^{\circ}, 90^{\circ}$ \\
\hline$Z$ & 4 \\
\hline$V\left(\AA^{3}\right)$ & $2975.9(5)$ \\
\hline Temperature (K) & $170.02 \mathrm{~K}$ \\
\hline Size $/ \mathrm{mm}^{3}$ & $0.05 \times 0.02 \times 0.006$ \\
\hline Density $\left(\mathrm{g} / \mathrm{cm}^{-3}\right)$ & 1.341 \\
\hline Measured reflections & 25403 \\
\hline Unique reflections & 5431 \\
\hline Parameters & 433 \\
\hline$R_{\text {int }}$ & 0.0469 \\
\hline$\theta$ range $\left(^{\circ}\right)$ & $4.584-55.040^{\circ}$ \\
\hline$R_{1}, w R_{2}$ & $0.0887,0.2551$ (Final $\mathrm{R}$ indices) \\
\hline$S(\mathrm{GOF})$ & 1.053 \\
\hline Max $/$ min res. dens. $\left(\mathrm{e} / \AA^{3}\right)$ & $0.672 /-0.264$ \\
\hline
\end{tabular}


Table S4 Crystal data and structure determination for $\mathbf{4}$

\begin{tabular}{|c|c|}
\hline Compound & 4 \\
\hline Chemical formula & $\mathrm{C}_{48} \mathrm{H}_{28}$ \\
\hline Formula mass & 604.70 \\
\hline Crystal system & Triclinic \\
\hline Space group & P-1 \\
\hline$\lambda(\AA)$ & 1.54184 \\
\hline$a, b, c(\AA)$ & $15.9694(9), 17.6913(7), 18.4228(5)$ \\
\hline$\alpha, \beta, \gamma\left({ }^{\circ}\right)$ & 78.853(3), 88.188(3), 69.169(4) \\
\hline Z & 1 \\
\hline$V\left(\AA^{3}\right)$ & $4768.9(4)$ \\
\hline Temperature (K) & $100 \mathrm{~K}$ \\
\hline Size $/ \mathrm{mm}^{3}$ & --- \\
\hline Density $\left(\mathrm{g} / \mathrm{cm}^{-3}\right)$ & 1.1646 \\
\hline Measured reflections & 70150 \\
\hline Unique reflections & 18942 \\
\hline Parameters & 898 \\
\hline$R_{\text {int }}$ & 0.0799 \\
\hline$\theta$ range $\left(^{\circ}\right)$ & $3.77-73.58$ \\
\hline$R_{1}, w R_{2}$ & $0.1271,0.3206$ (Final R indices) \\
\hline$S(\mathrm{GOF})$ & 1.037 \\
\hline Max/min res. dens. $\left(\mathrm{e} / \AA^{3}\right)$ & $1.68 /-1.00$ \\
\hline
\end{tabular}


Table S5 Crystal data and structure determination for $\mathbf{5}$

\begin{tabular}{|c|l|}
\hline Compound & \\
\hline Chemical formula & $\mathrm{C}_{48} \mathrm{H}_{24}$ \\
\hline Formula mass & 600.67 \\
\hline Crystal system & Monoclinic \\
\hline Space group & $\mathrm{C} 12 / \mathrm{c} 1$ \\
\hline$\lambda(\AA)$ & 1.34139 \\
\hline$a, b, c(\AA)$ & $30.271(15), 3.941(2), 26.334(14)$ \\
\hline$\alpha, \beta, \gamma\left({ }^{\circ}\right)$ & $90^{\circ}, 119.498(16)^{\circ}, 90^{\circ}$ \\
\hline$Z$ & 4 \\
\hline$V\left(\AA^{3}\right)$ & $2734(2)$ \\
\hline Temperature $(\mathrm{K})$ & $173.0 \mathrm{~K}$ \\
\hline Size $/ \mathrm{mm}^{3}$ & $0.05 \times 0.02 \times 0.01$ \\
\hline Density $\left(\mathrm{g} / \mathrm{cm}^{-3}\right)$ & 1.459 \\
\hline Measured reflections & 9305 \\
\hline Unique reflections & 2544 \\
\hline Parameters & 217 \\
\hline$R_{\text {int }}$ & 0.0946 \\
\hline$\theta$ range $\left({ }^{\circ}\right)$ & $3.355-55.590^{\circ}$ \\
\hline$R_{1}, w R_{2}$ & $0.0884,0.2220$ (Final R indices) \\
\hline$S(\mathrm{GOF})$ & 0.985 \\
\hline Max/min res. dens. $\left(\mathrm{e} / \AA^{3}\right)$ & $0.280 / 0.356$ \\
\hline & \\
\hline
\end{tabular}


Table S6 Crystal data and structure determination for $\mathbf{6}$

\begin{tabular}{|c|l|}
\hline Compound & \\
\hline Chemical formula & $\mathrm{C}_{48} \mathrm{H}_{24}$ \\
\hline Formula mass & 600.67 \\
\hline Crystal system & Monoclinic \\
\hline Space group & $\mathrm{C} 12 / \mathrm{c} 1$ \\
\hline$\lambda(\AA)$ & 1.34139 \\
\hline$a, b, c(\AA)$ & $23.116(4), 18.273(3), 17.154(2)$ \\
\hline$\alpha, \beta, \gamma\left({ }^{\circ}\right)$ & $90^{\circ}, 90.060(12)^{\circ}, 90^{\circ}$ \\
\hline$Z$ & 8 \\
\hline$V\left(\AA^{3}\right)$ & $7246(2)$ \\
\hline Temperature $(\mathrm{K})$ & $193.0 \mathrm{~K}$ \\
\hline Size $/ \mathrm{mm}^{3}$ & $0.02 \times 0.02 \times 0.01$ \\
\hline Density $\left(\mathrm{g} / \mathrm{cm}^{-3}\right)$ & 1.101 \\
\hline Measured reflections & 18194 \\
\hline Unique reflections & 5522 \\
\hline Parameters & 433 \\
\hline$R_{\text {int }}$ & 0.0886 \\
\hline$\theta$ range $\left({ }^{\circ}\right)$ & $3.327-49.649^{\circ}$ \\
\hline$R_{1}, w R_{2}$ & $0.1279,0.3061($ Final R indices $)$ \\
\hline$S(\mathrm{GOF})$ & 1.096 \\
\hline Max/min res. dens. $\left(\mathrm{e} / \AA^{3}\right)$ & $0.358 /-0.248$ \\
\hline & \\
\hline
\end{tabular}




\section{Optical and electronic spectra, frontier orbitals energies (Figure S7, Table S7)}
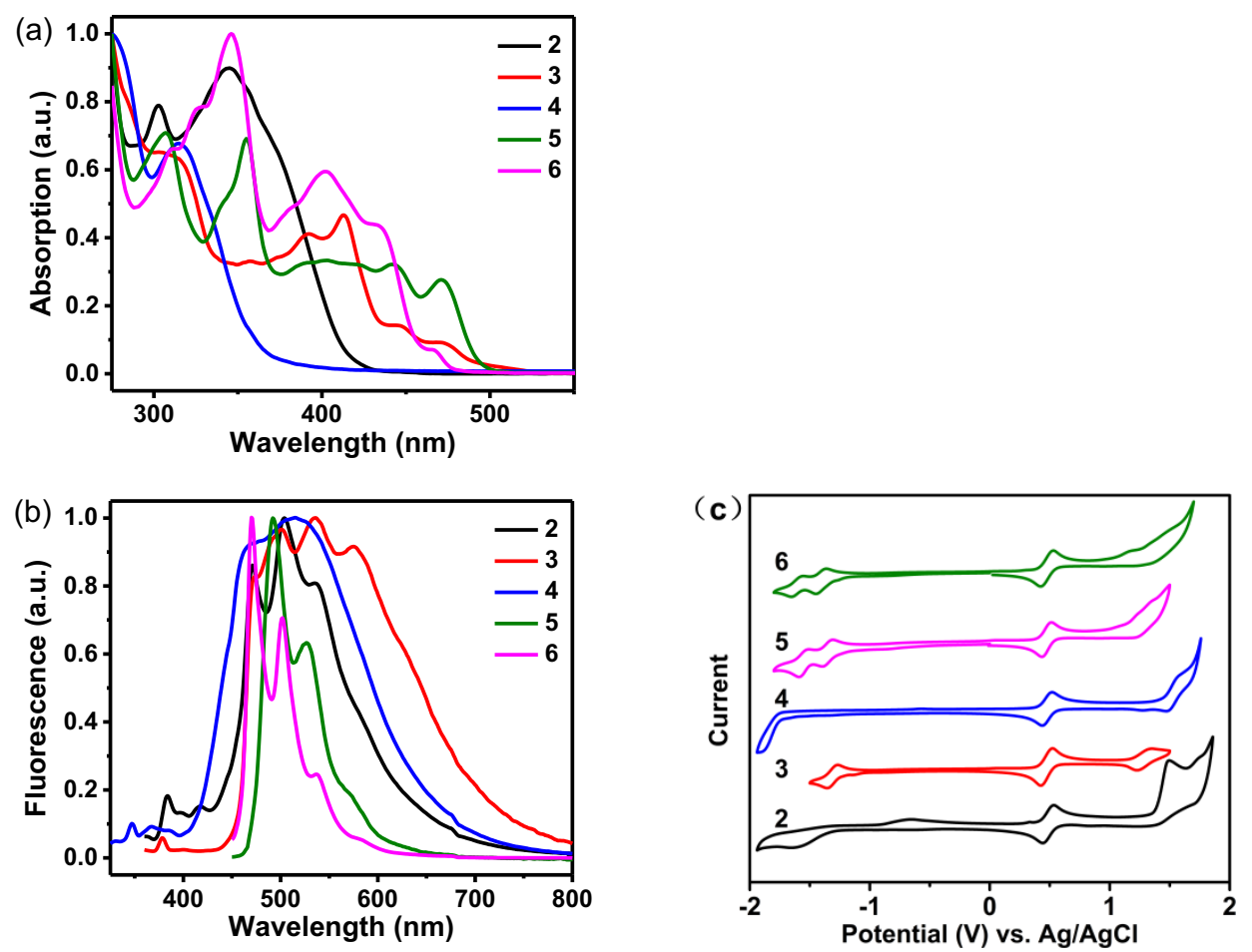

Figure S7. (a) Absorption and (b) emission spectra of 2-6 in cyclohexane $\left(1 \times 10^{-5} \mathrm{M}\right)$ excited at wavelengths of $350 \mathrm{~nm}, 350$ $\mathrm{nm}, 315 \mathrm{~nm}, 440 \mathrm{~nm}$ and $440 \mathrm{~nm}$, respectively. (All data were normalized for comparison). (c) Cyclic Voltammograms of compounds 2 (black), 3 (red), 4 (blue), 5 (purple) and 6 (green) with $\mathrm{Ag} / \mathrm{AgCl}$ as reference electrode and $\mathrm{Bu}_{4} \mathrm{NPF}_{6}(0.1 \mathrm{M})$ as the supporting electrolyte in $\mathrm{CH}_{2} \mathrm{Cl}_{2}$. Ferrocene was used as an internal standard.

Table S7. The absorption, cyclic voltammetry and frontier orbitals energies parameters of compunds 2-6.

\begin{tabular}{|c|c|c|c|c|c|c|c|c|c|c|c|c|}
\hline \multirow[t]{2}{*}{ Compound } & \multirow{2}{*}{$\begin{array}{l}\mathbf{E}_{\mathrm{Fe}} \\
(\mathrm{V})\end{array}$} & \multirow{2}{*}{$\begin{array}{l}\mathbf{E}_{\mathbf{r} 1} \\
(\mathrm{~V})\end{array}$} & \multirow{2}{*}{$\begin{array}{l}\mathbf{E}_{\mathbf{r} 2} \\
(\mathrm{~V})\end{array}$} & \multirow{2}{*}{$\begin{array}{l}\mathbf{E}_{01} \\
(\mathbf{V})\end{array}$} & \multirow{2}{*}{$\begin{array}{c}\lambda_{\max } \\
\mathbf{n m}\end{array}$} & \multicolumn{2}{|c|}{$\begin{array}{c}\text { HOMO } \\
(\mathrm{eV})\end{array}$} & \multicolumn{2}{|c|}{$\begin{array}{c}\text { LUMO } \\
(\mathrm{eV})\end{array}$} & \multicolumn{3}{|c|}{$\begin{array}{l}E_{\text {gap }} \\
(\mathrm{eV})\end{array}$} \\
\hline & & & & & & $\mathbf{C V}$ & Cal. $^{a}$ & $\mathbf{C V}$ & Cal. $^{a}$ & $\mathbf{C V}$ & Cal. $^{a}$ & Opt. \\
\hline 2 & 0.47 & - & - & - & 377 & - & -5.83 & - & -2.26 & - & 3.52 & 3.28 \\
\hline 3 & 0.47 & -1.31 & - & 1.28 & 473 & -5.61 & -5.57 & -3.02 & -2.51 & 2.59 & 3.06 & 2.62 \\
\hline 4 & 0.47 & - & - & 1.55 & 335 & -5.88 & -5.72 & - & -1.91 & - & 3.75 & 3.81 \\
\hline 5 & 0.47 & -1.35 & -1.55 & - & 470 & - & -5.55 & -2.98 & -2.53 & - & 2.99 & 3.02 \\
\hline 6 & 0.47 & -1.40 & -1.60 & - & 446 & - & -5.69 & -2.93 & -2.46 & - & 3.23 & 2.66 \\
\hline
\end{tabular}

${ }^{a}$ Calculated at B3LYP-D3/6-311+G(2d,p) in gas phase. 
4 NICS(1) values of compounds 2-6 (Figure S8)

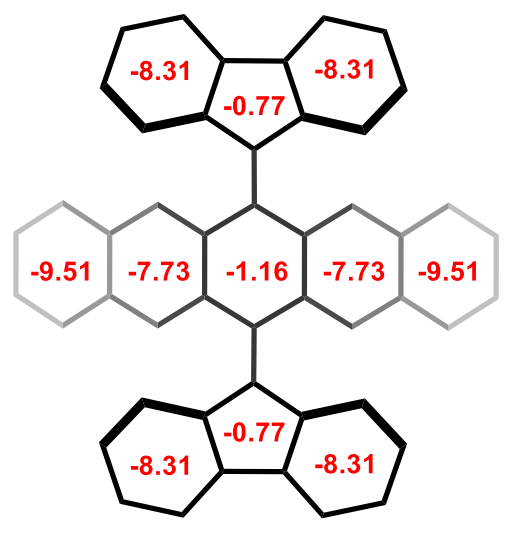

2

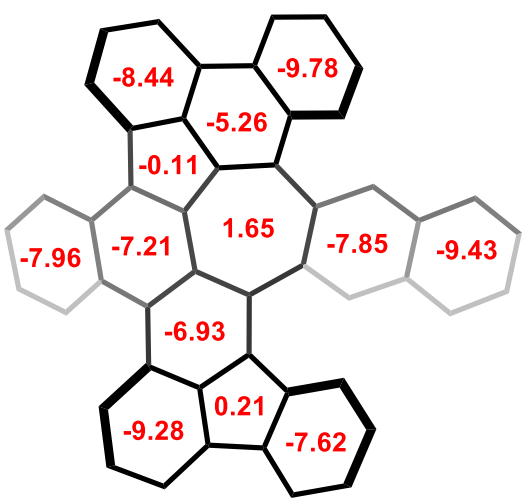

3

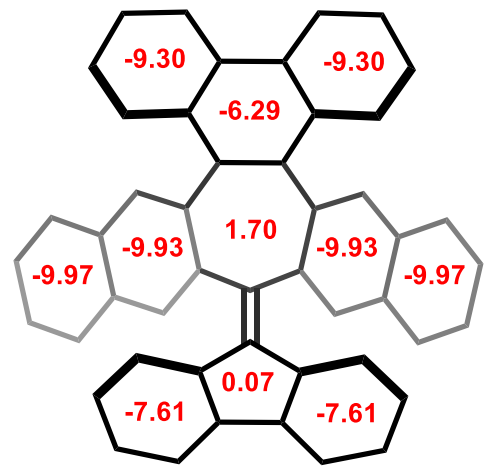

4

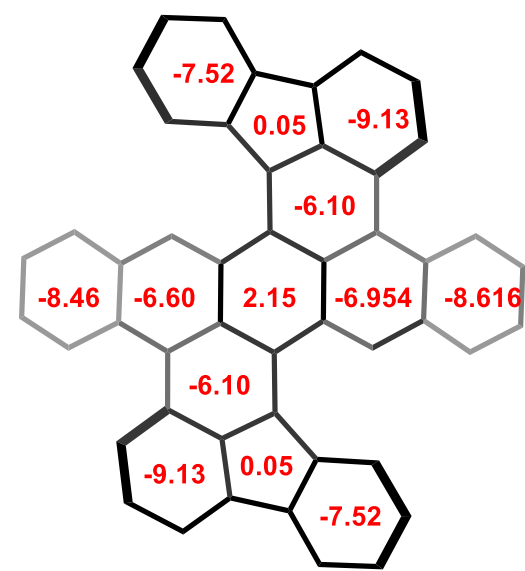

5

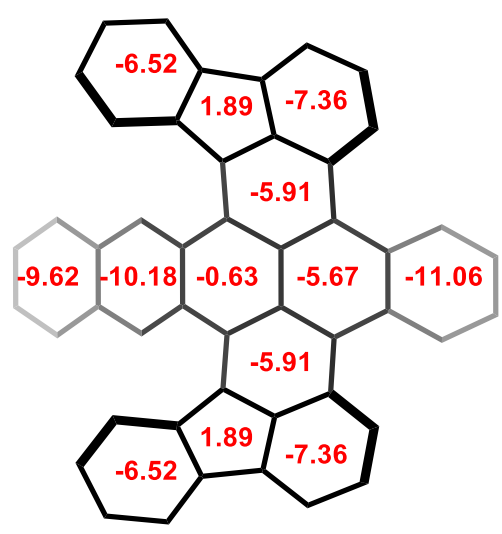

6

Figure S8. NICS(1) values of compounds 2-6, calculated at the GIAO-B3LYP/6-311+G(2d,p)//B3LYP-D3/6-311+G(2d,p) level of theory in gas phase. 


\section{Mechanistic explorations of the first rearrangement}

\subsection{Reaction pathways of the first rearrangement (Figure S9-S12, Table S8)}

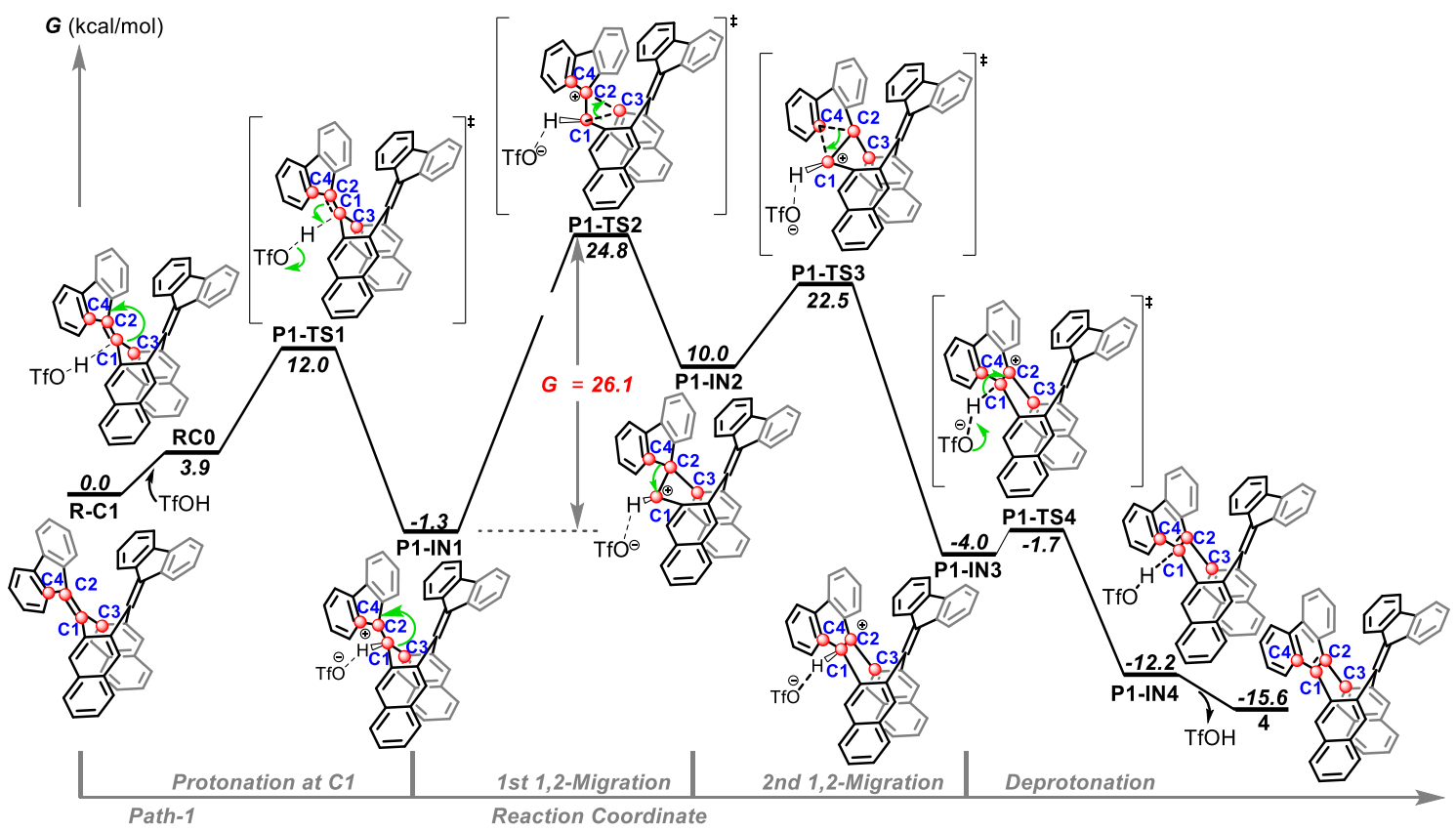

Figure S9. Free energy surface of Path-1 (P1), with a barrier 26.1 kcal/mol. Calculated at B3LYP-D3/6-31+G*/(SMD, solvent $=$ dichloromethane). Please refer to Figure S10 for 3D structures. 

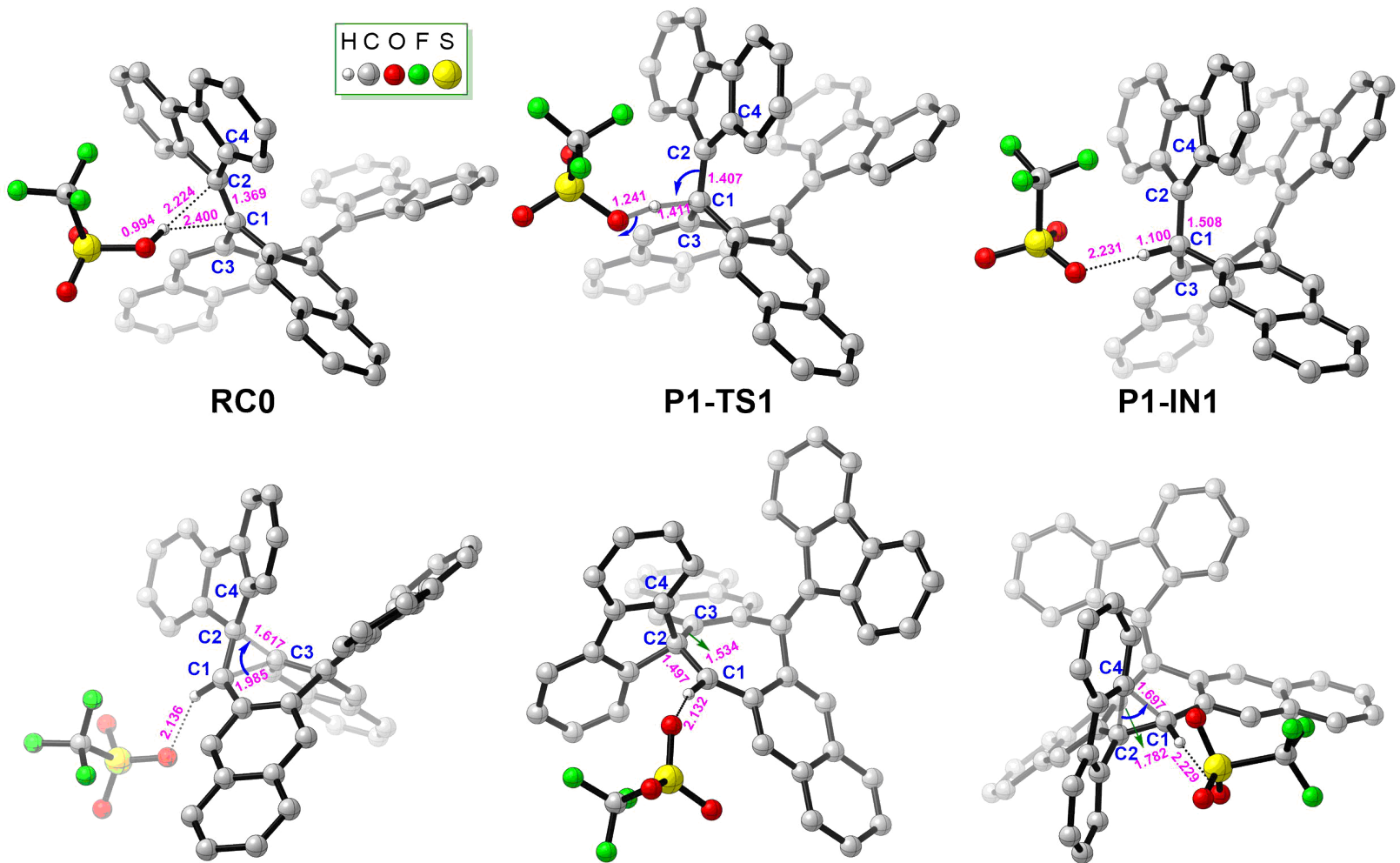

P1-IN1

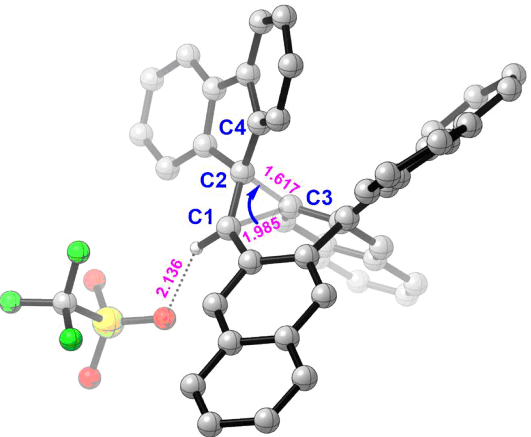

P1-TS2

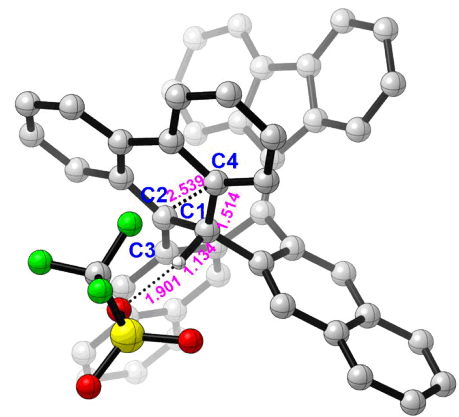

P1-IN3
P1-IN2

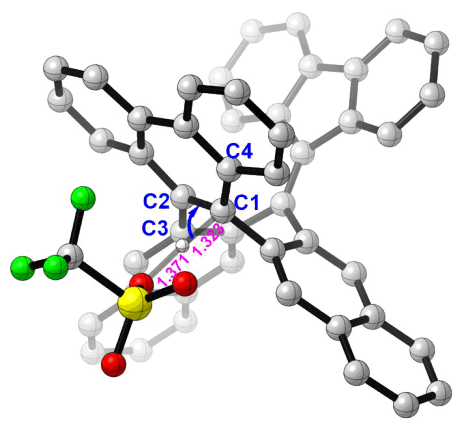

P1-TS4

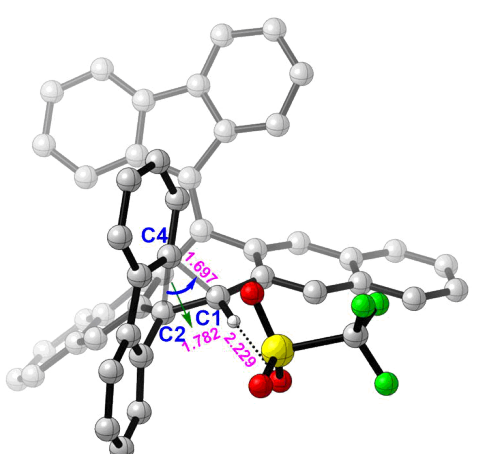

P1-TS3

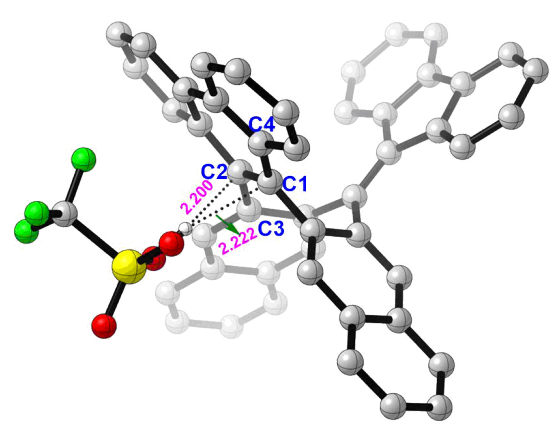

P1-IN4

Figure S10. 3D structures of intermediates and transition states along Path-1 (P1) in Figure S9. Selected bond lengths (unit: A) are shown. Hydrogens except that from TfOH are omitted for clarity. 


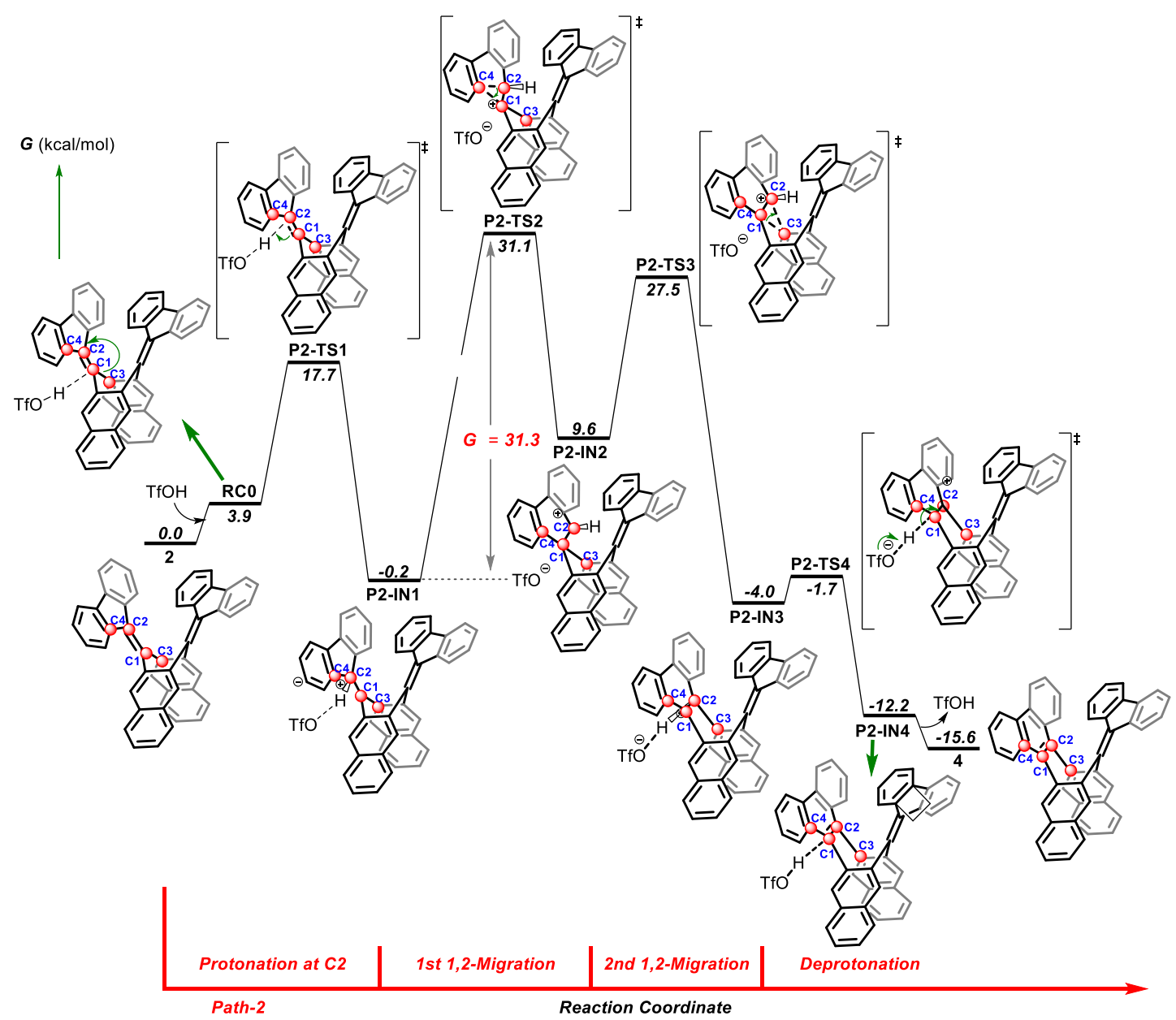

Figure S11. Free energy surface of Path-2 (P2), barrier of which is $31.3 \mathrm{kcal} / \mathrm{mol}$. Calculated at B3LYP-D3/6-31+G*/(SMD, solvent $=$ dichloromethane). Please refer to Figure S12 for 3D structures. 


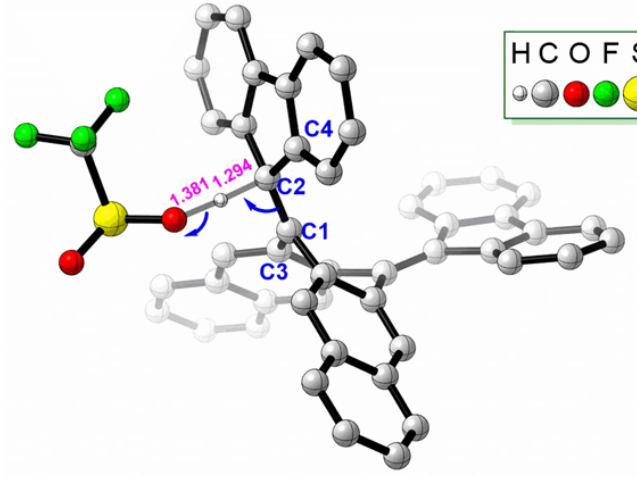

P2-TS1

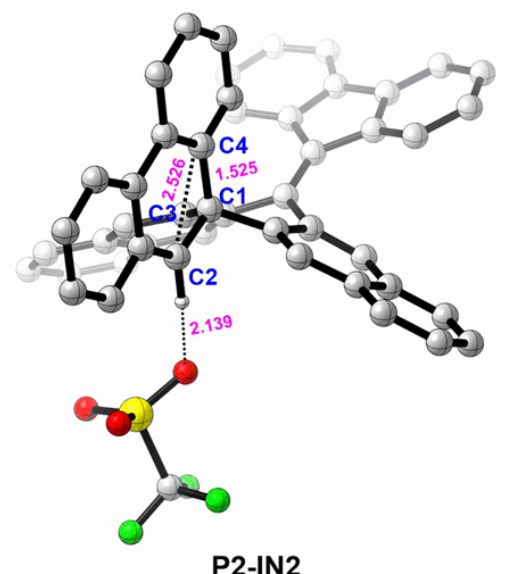

P2-IN2

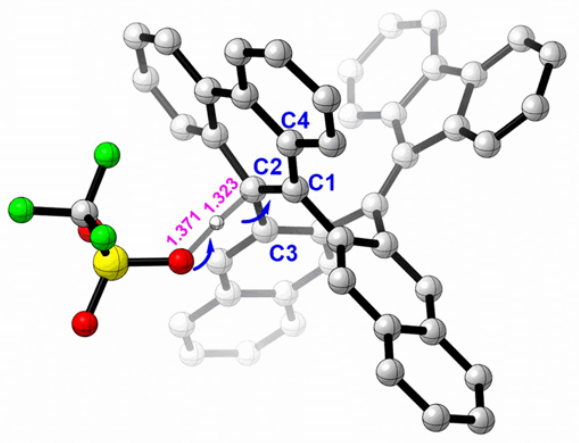

P2-TS4

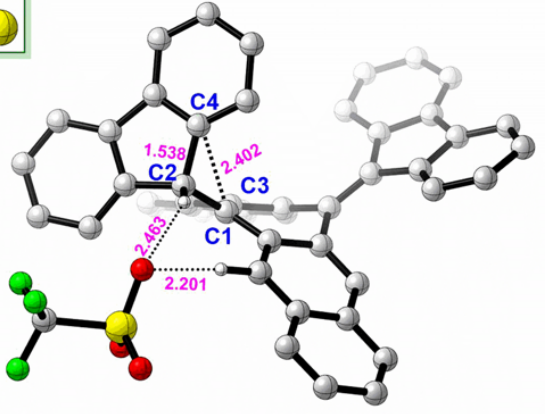

P2-IN1

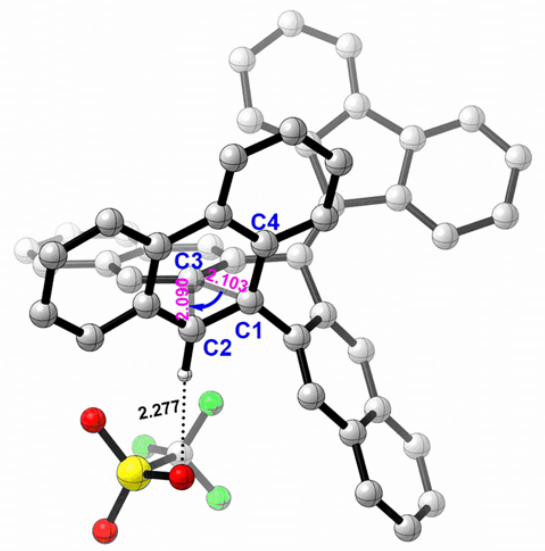

P2-TS3

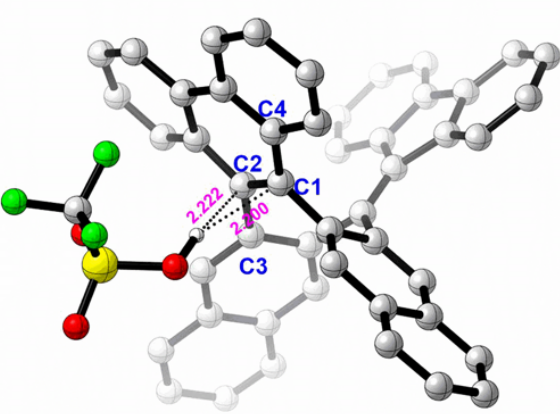

P2-IN4

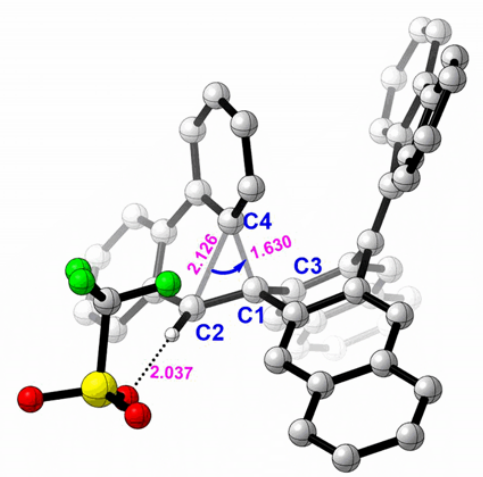

P2-TS2

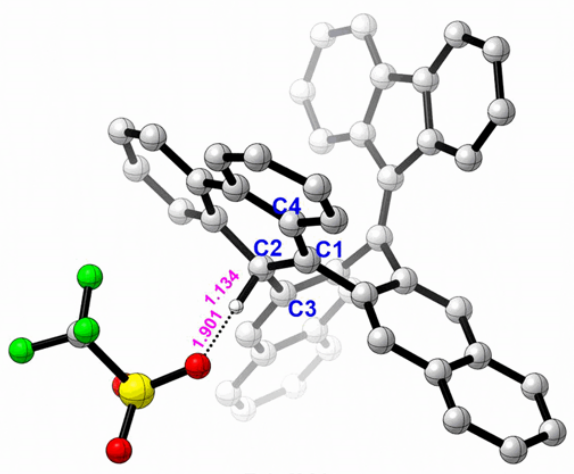

P2-IN3

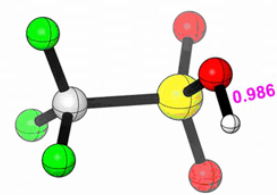

$\mathrm{TfOH}$

Figure S12. 3D structures of intermediates and transition states along Path-2 (P2) in Figure S11. Selected bond lengths (unit: $\AA)$ are shown. Hydrogens except that from $\mathrm{TfOH}$ are omitted for clarity. 
Table S8. The absolute energy (electronic and Gibbs free energy, i.e. $\mathbf{E}$ and $\mathbf{G}$, unit: Hartree) and relative energy ( $\mathbf{\Delta E}$ and $\boldsymbol{\Delta G}$, unit: $\mathrm{kcal} / \mathrm{mol}$ ) for the different reactant conformations, TfOH, and intermediates and transition states along pathway $\mathbf{P 1}$ and $\mathbf{P 2}$ in Figure S9-S12.

\begin{tabular}{|c|c|c|c|c|c|c|c|}
\hline Entry & $\Delta G_{B 3}{ }^{a}$ & $\Delta G_{B 3-s p}{ }^{b}$ & $\Delta G_{M 06-s p}{ }^{c}$ & $\mathbf{E}_{\mathrm{B} 3}{ }^{a}$ & $\mathrm{G}_{\mathrm{B} 3}{ }^{a}$ & $E_{B 3-s p}^{b}$ & $\mathbf{E}_{\text {M06-sp }}{ }^{c}$ \\
\hline 2 & 0.0 & 0.0 & 0.0 & -1846.211257 & -1845.675876 & -1846.579685 & -1845.040466 \\
\hline 4 & -15.6 & -15.7 & -17.0 & -1846.237553 & -1845.700783 & -1846.606044 & -1845.068906 \\
\hline TfOH & -- & -- & -- & -962.048836 & -962.045229 & -962.230971 & -961.973858 \\
\hline R-CO & 3.9 & 3.3 & 6.3 & -2808.274463 & -2807.714969 & -2808.825966 & -2807.024866 \\
\hline P1-TS1 & 12.0 & 12.1 & 15.6 & -2808.256868 & -2807.701934 & -2808.807315 & -2807.005452 \\
\hline P1-INT1 & -1.3 & 0.6 & 2.9 & -2808.279393 & -2807.723252 & -2808.826922 & -2807.026884 \\
\hline P1-TS2 & 24.8 & 27.2 & 27.8 & -2808.242698 & -2807.681539 & -2808.789498 & -2806.992156 \\
\hline P1-INT2 & 10.0 & 11.9 & 14.3 & -2808.265623 & -2807.705160 & -2808.813102 & -2807.013018 \\
\hline P1-TS3 & 22.5 & 24.9 & 23.7 & -2808.245314 & -2807.685242 & -2808.792005 & -2806.997647 \\
\hline P1-INT3 & -4.0 & -2.5 & -0.5 & -2808.285436 & -2807.727509 & -2808.833531 & -2807.034098 \\
\hline P1-TS4 & -1.7 & -1.6 & 1.9 & -2808.280260 & -2807.723885 & -2808.830575 & -2807.028757 \\
\hline P1-INT4 & -12.2 & -12.9 & -11.3 & -2808.302113 & -2807.740568 & -2808.853810 & -2807.054866 \\
\hline P2-TS1 & 17.7 & 17.7 & 22.4 & -2808.249139 & -2807.692916 & -2808.799674 & -2806.995859 \\
\hline P2-INT1 & -0.2 & 1.9 & 5.4 & -2808.281427 & -2807.721393 & -2808.828664 & -2807.026836 \\
\hline P2-TS2 & 31.1 & 32.5 & 33.6 & -2808.233107 & -2807.671570 & -2808.781356 & -2806.983326 \\
\hline P2-INT2 & 9.6 & 11.7 & 14.2 & -2808.266482 & -2807.705759 & -2808.813778 & -2807.013429 \\
\hline P2-TS3 & 27.5 & 29.0 & 31.5 & -2808.238449 & -2807.677303 & -2808.786665 & -2806.986244 \\
\hline P2-INT3 & -4.0 & -2.5 & -0.5 & -2808.285436 & -2807.727509 & -2808.833531 & -2807.034098 \\
\hline P2-TS4 & -1.7 & -1.6 & 1.9 & -2808.280260 & -2807.723885 & -2808.830575 & -2807.028757 \\
\hline P2-INT4 & -12.2 & -12.9 & -11.3 & -2808.302113 & -2807.740568 & -2808.853810 & -2807.054866 \\
\hline
\end{tabular}

${ }^{a} \mathbf{E}_{\mathrm{B} 3}, \mathbf{G}_{\mathrm{B} 3}$, and $\boldsymbol{\Delta} \boldsymbol{G}_{\boldsymbol{B} 3}$ are obtained at B3LYP-D3/6-31+G*level;

${ }^{b} \mathbf{E}_{\mathbf{B} 3 \text {-sp }}$, and $\boldsymbol{\Delta} \boldsymbol{G}_{\boldsymbol{B} 3 \text {-sp }}$ are obtained at B3LYP-D3/6-311++G**//B3LYP-D3/6-31+G* level;

${ }^{c} \mathbf{E}_{\mathbf{M 0 6}-\mathrm{sp}}$, and $\boldsymbol{\Delta} \boldsymbol{G}_{\boldsymbol{M} \text { 06-sp }}$ are obtained at M06/6-311++G**//B3LYP-D3/6-31+G* level. All these calculation are in solution (SMD, solvent $=$ dichloromethane). 


\subsection{IRC calculations of P1 pathway (Figure S13-S16)}
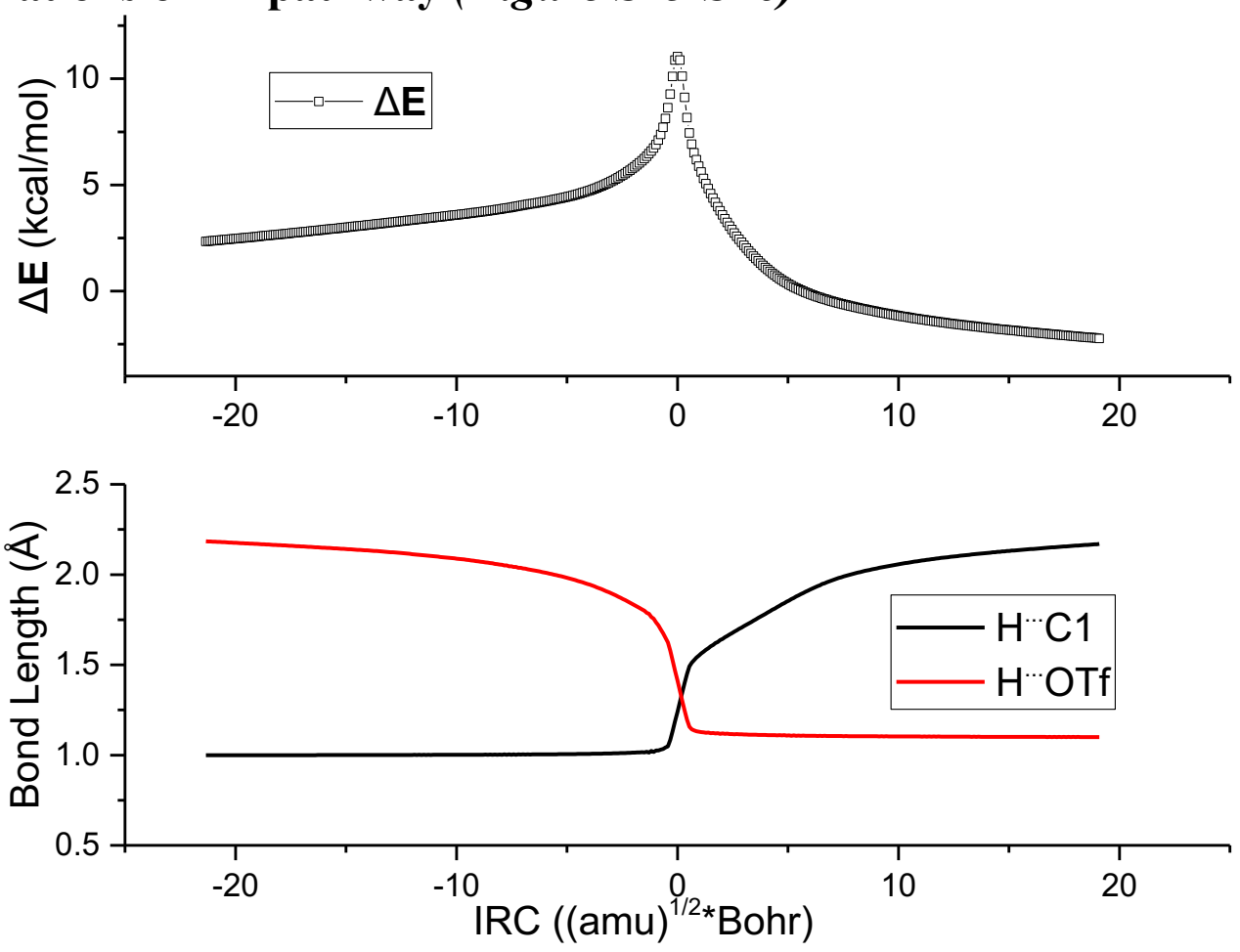

Figure S13. Results of IRC calculation on P1-TS1.
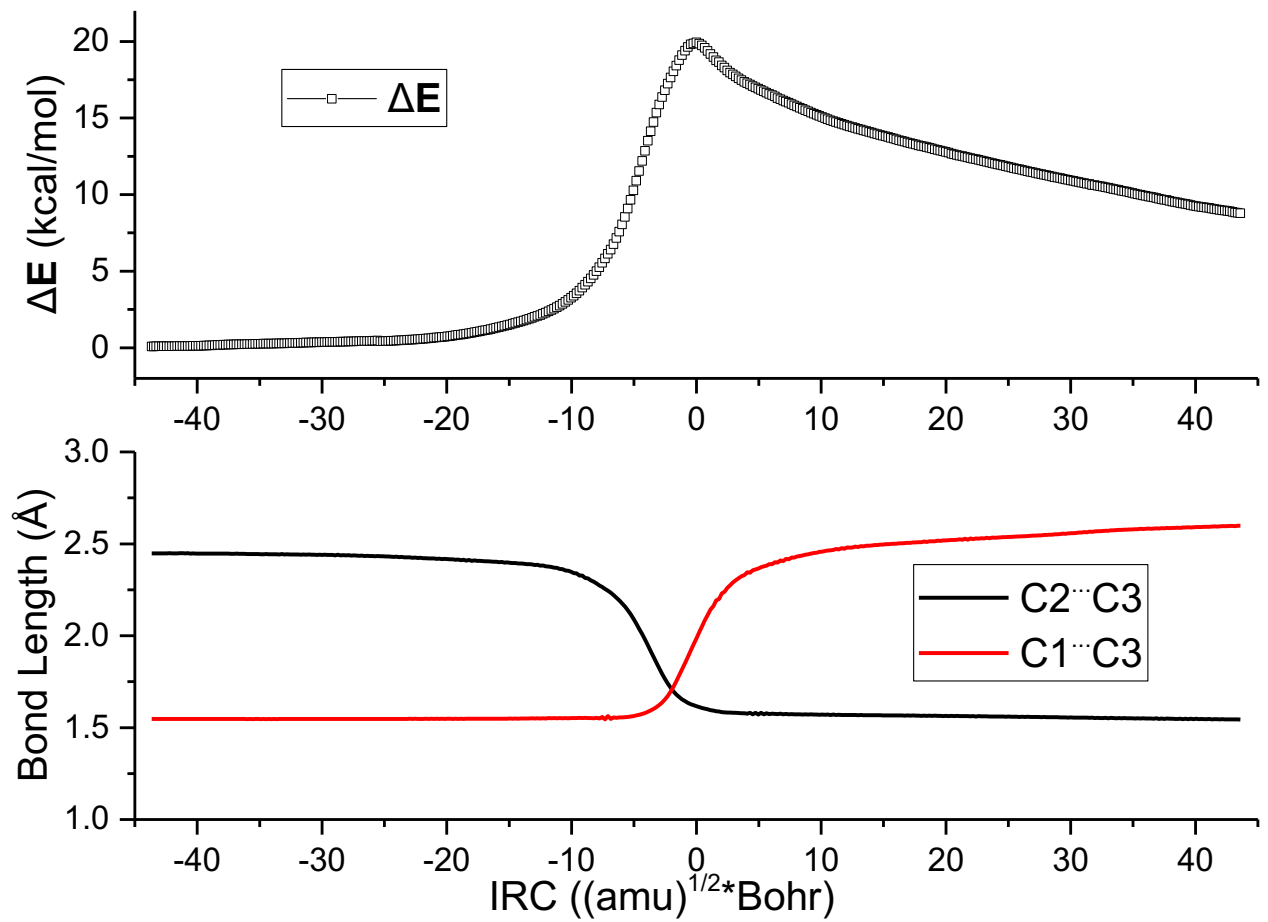

Figure S14. Results of IRC calculation on P1-TS2. 


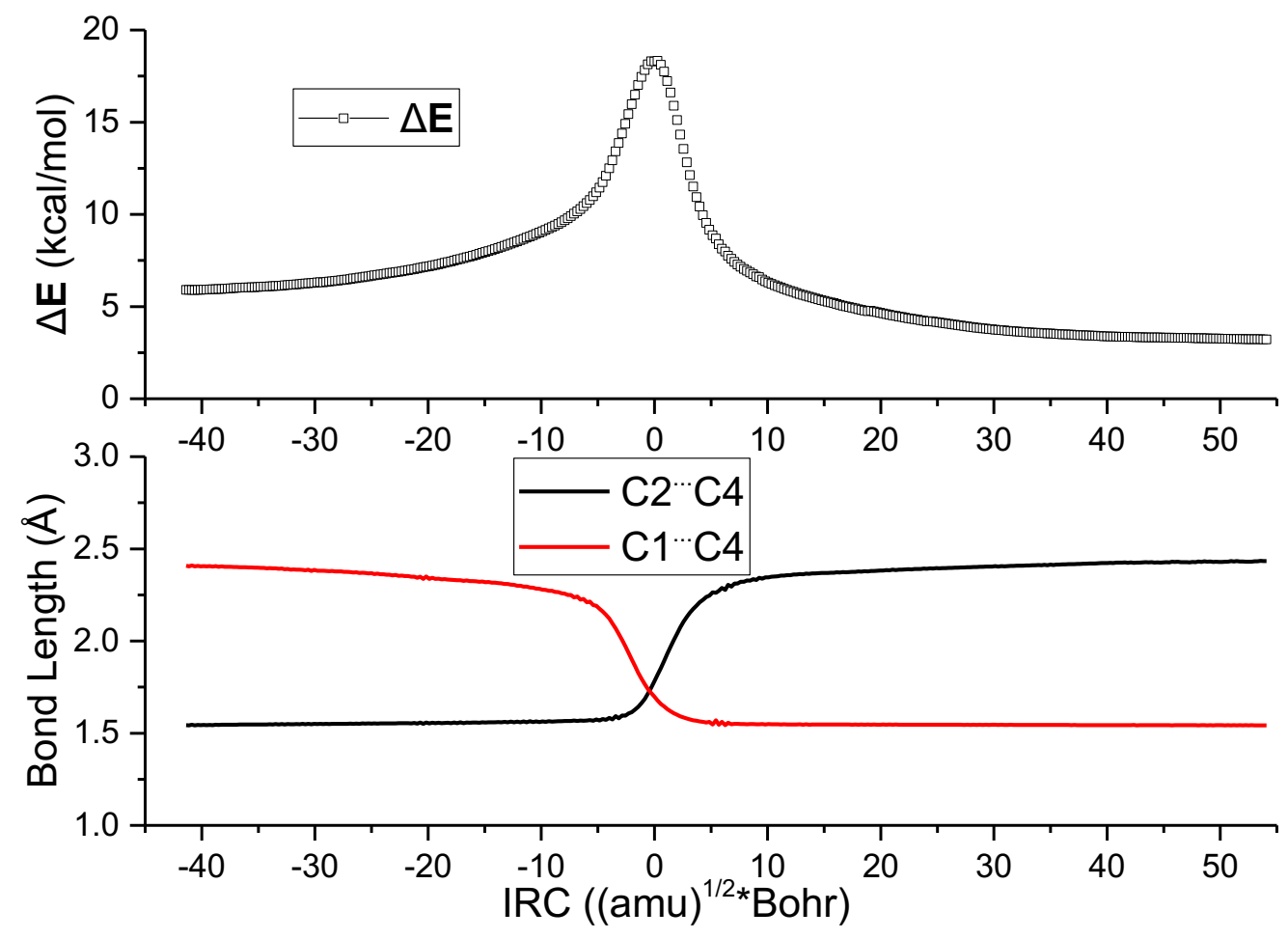

Figure S15. Results of IRC calculation on P1-TS3.

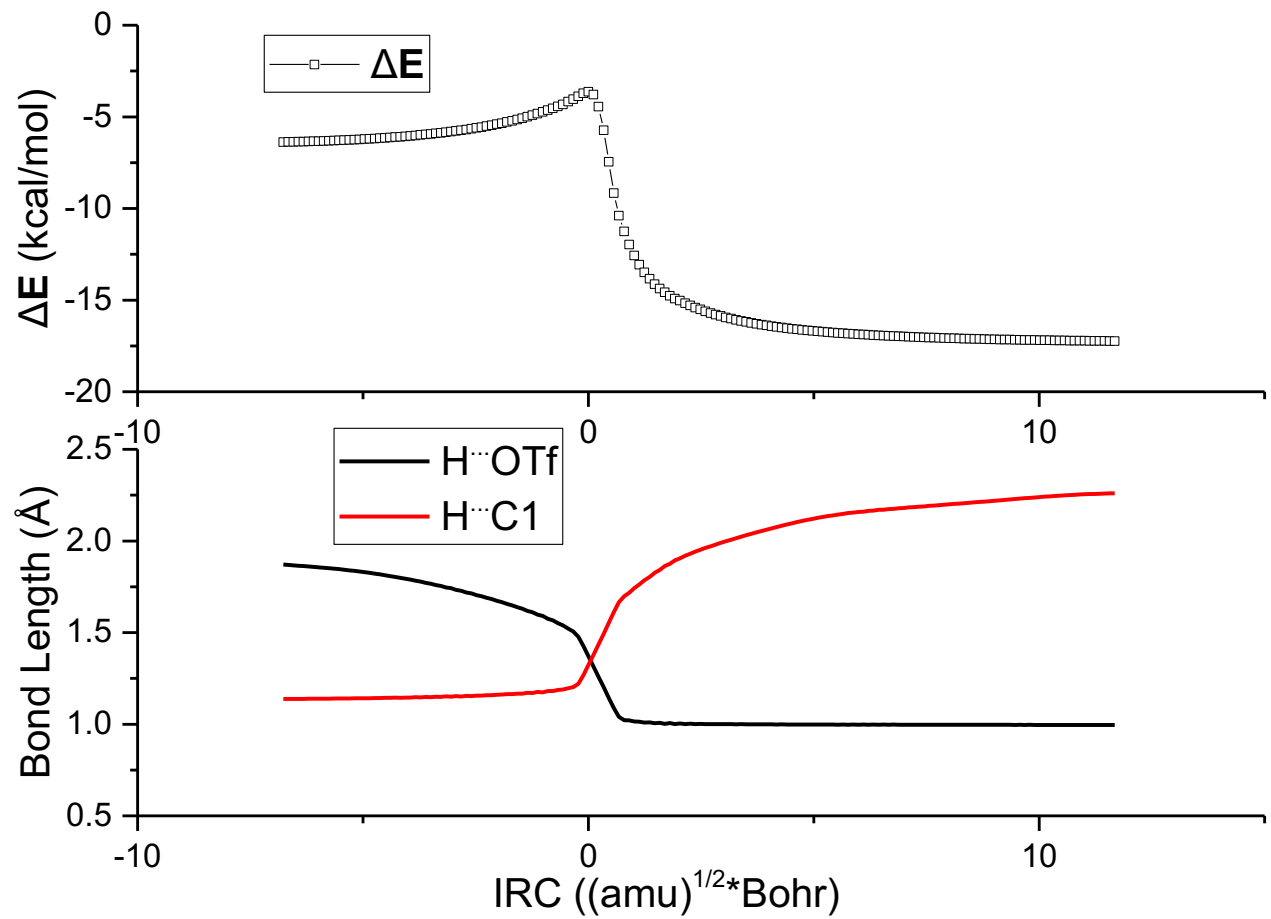

Figure S16. Results of IRC calculation on P1-TS4. 


\subsection{Calculations on the possible second rearrangement of 4 (Figure S17-S19, Table}

S9)

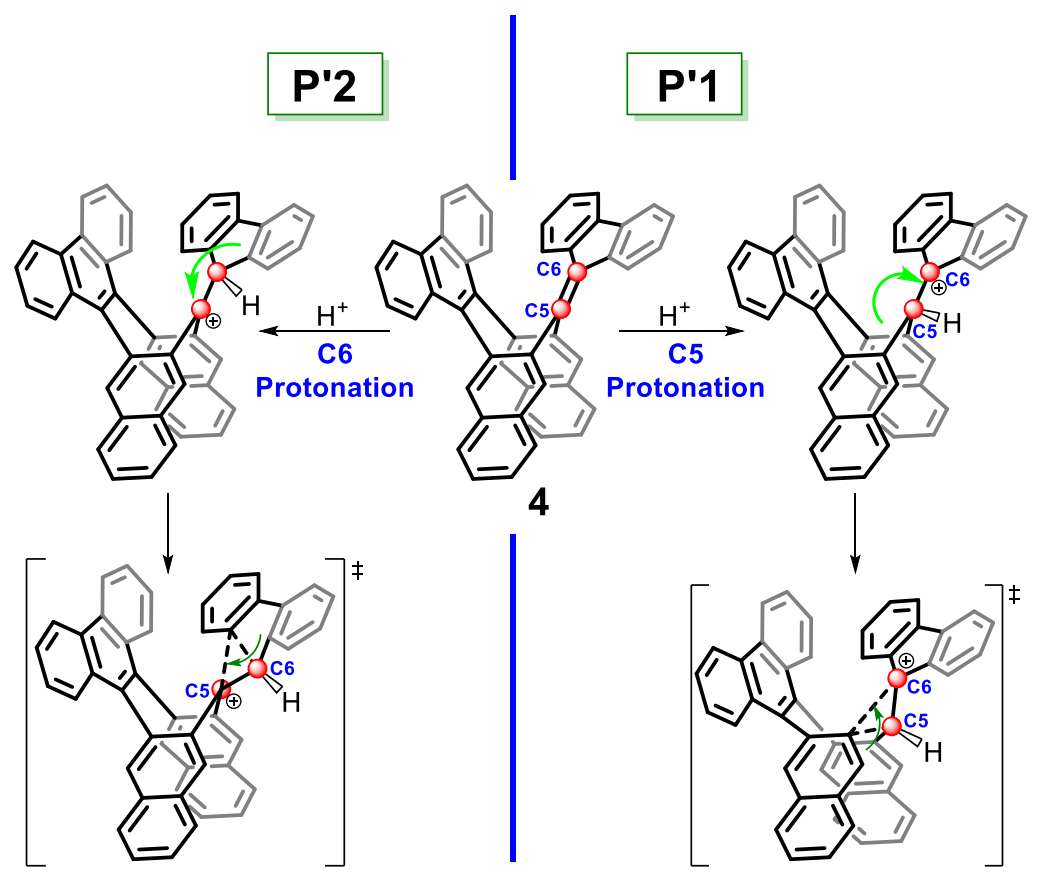

Figure S17. Partial further rearrangement pathways $\mathbf{P}^{\prime} \mathbf{1}$ and $\mathbf{P}^{\prime} \mathbf{2}$ from product $\mathbf{3}$.

As shown in Scheme 1 of the main text, the rearrangement reaction occurs only on one olefinic $\mathrm{C}=\mathrm{C}$ double bond of compound 2, leaving the other one in compound 4 unchanged. Further DFT calculations were performed to investigate the free energy barrier for the further rearrangement on the other $\mathrm{C}=\mathrm{C}$ double bond in compound $\mathbf{4}$. Partial rearrangement along pathways $\mathbf{P}^{\prime} \mathbf{1}$ and $\mathbf{P}^{\prime} \mathbf{2}$ as shown in Figure S17 are calculated. 


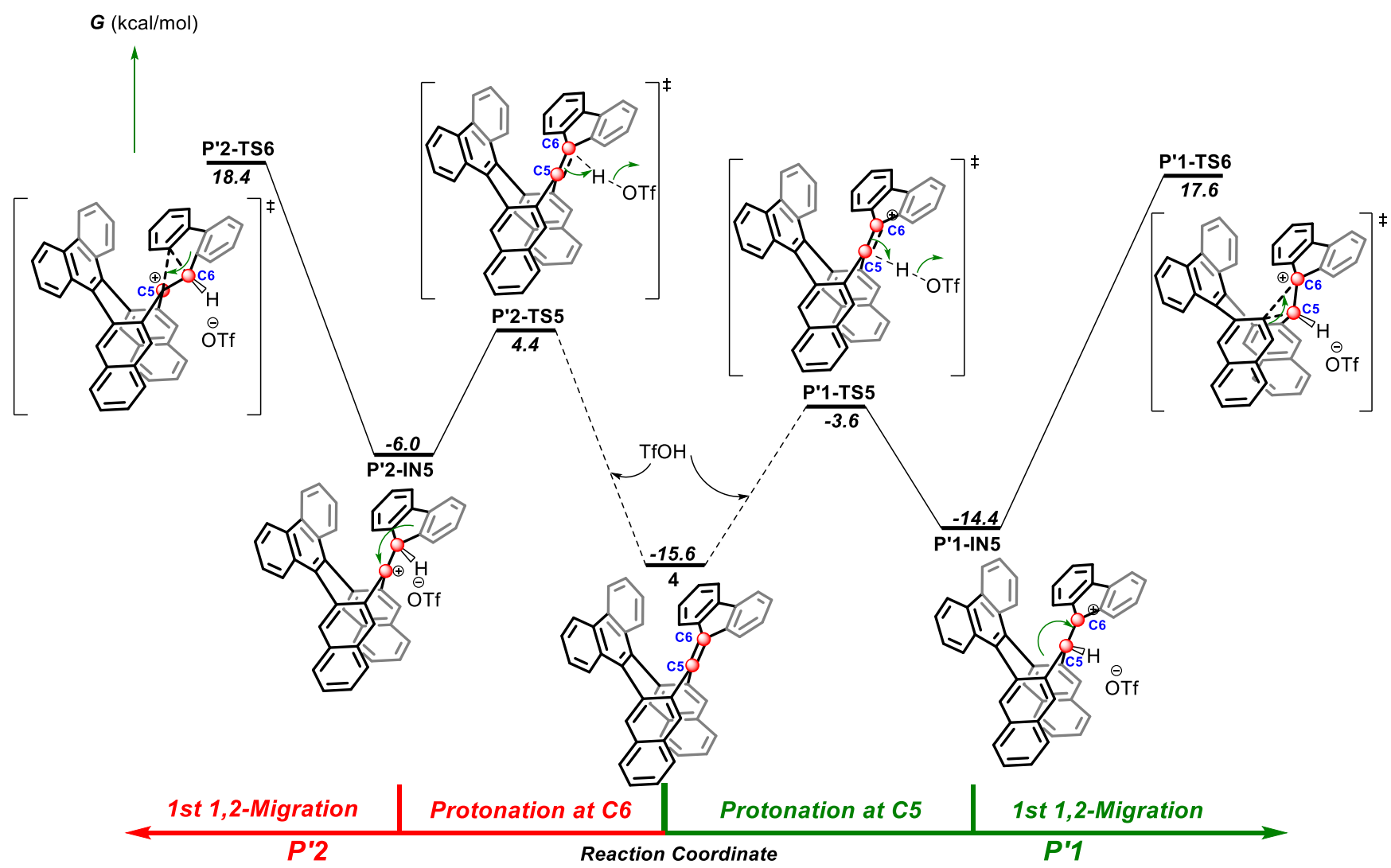

Figure S18. Free energy surface of $\mathbf{P}^{\prime} \mathbf{1}$ and $\mathbf{P}^{\prime} \mathbf{2}$, with barriers $33.2 \mathrm{kcal} / \mathrm{mol}$ and $34.0 \mathrm{kcal} / \mathrm{mol}$. Calculated at B3LYP-D3/6-31+G*/(SMD, solvent=dichloromethane). Please refer to Figure S19 for 3D structures.

The calculation results in Figure S18 suggested that after possible initial protonation of the $\mathrm{C}=\mathrm{C}$ double bond at either C5 (P'1-TS5, -3.6 kcal/mol) or C6 (P'2-TS5, $4.4 \mathrm{kcal} / \mathrm{mol})$, the following rearrangements P'1-TS6 $(17.6 \mathrm{kcal} / \mathrm{mol})$ and P'2-TS6 $(18.4 \mathrm{kcal} / \mathrm{mol})$ have very high energy barriers of $33.2 \mathrm{kcal} / \mathrm{mol}$ and $34.0 \mathrm{kcal} / \mathrm{mol}$ relative to compound 4, respectively. These energy barriers are too high when compared with the first rearrangement at room temperature (Figure 3(b) in the main text and Figure S9) and hence prevent the second rearrangement to occur under our experimental conditions (Scheme 1(c) in the main text). 

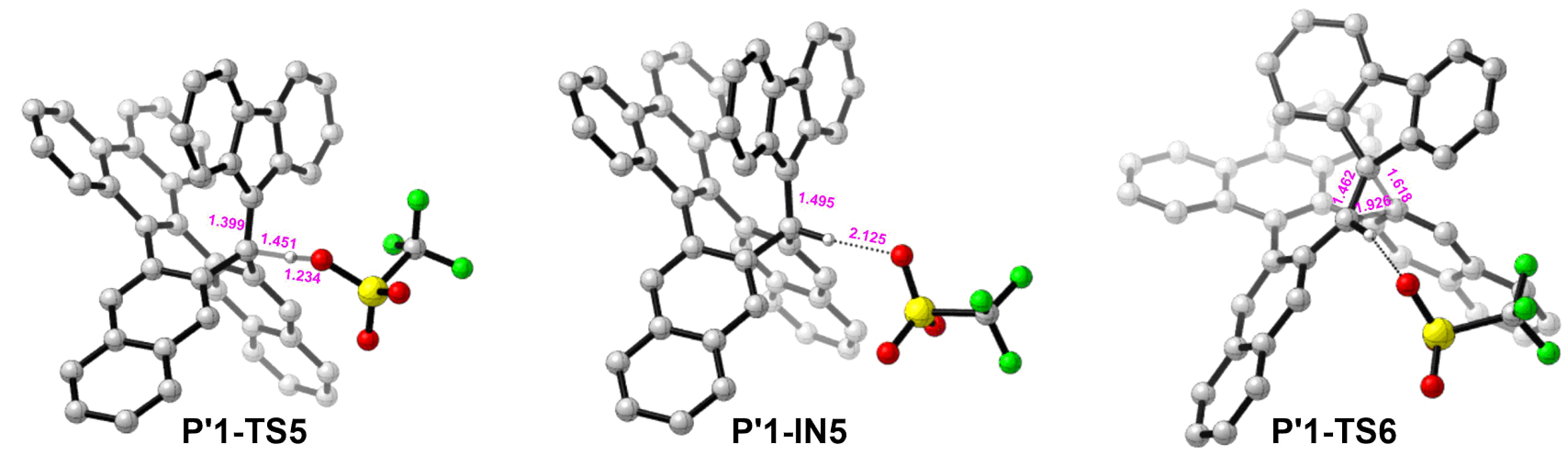

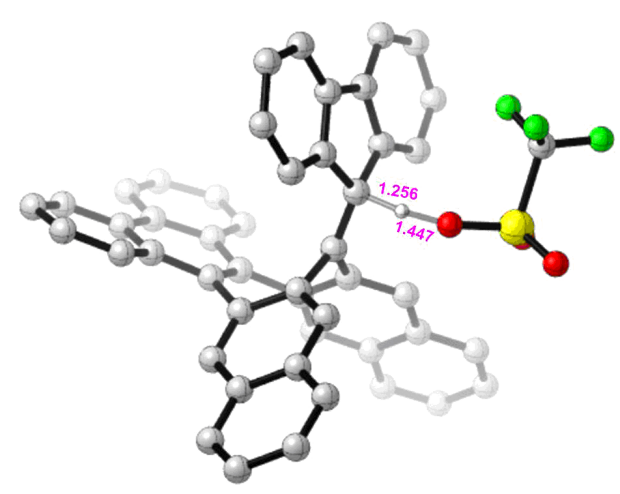

P'2-TS5

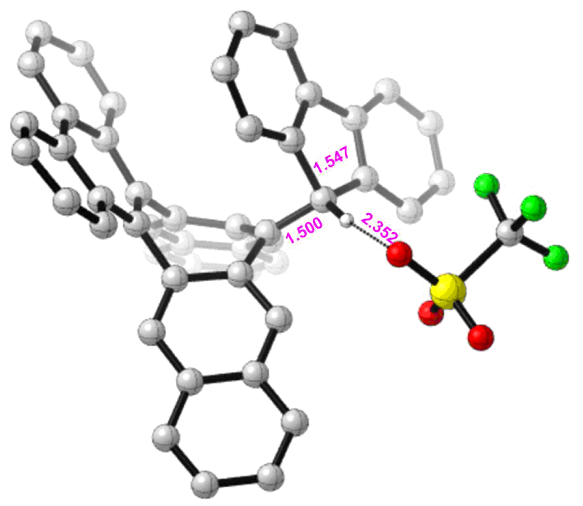

P'2-IN5

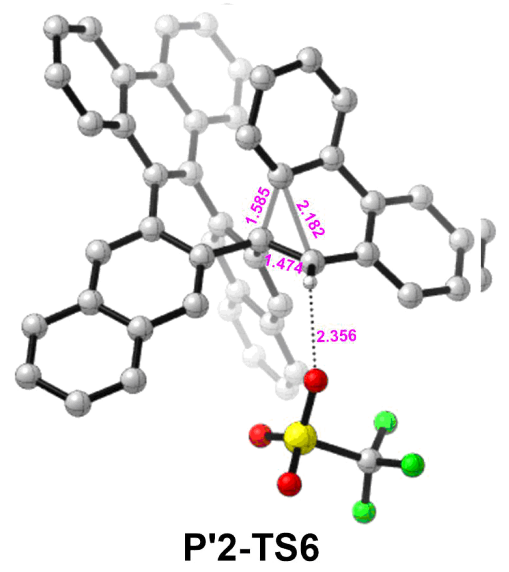

Figure S19. 3D structures of intermediates and transition states along pathways $\mathbf{P}^{\prime} \mathbf{1}$ and $\mathbf{P}^{\prime} \mathbf{2}$ (in Figure S18) with experimental compound $\mathbf{4}$ as the reactant. Selected bond lengths (unit: $\AA$ ) are shown. Hydrogens except that from TfOH are omitted for clarity. 
Table S9. The absolute energy (electronic and Gibbs free energy, i.e. $\mathbf{E}$ and $\mathbf{G}$, unit: Hartree) and relative energy ( $\Delta \mathbf{E}$ and $\boldsymbol{\Delta G}$, unit: $\mathrm{kcal} / \mathrm{mol}$ ) for intermediates and transition states along pathway $\mathbf{P}^{\prime} \mathbf{1}$ and $\mathbf{P}^{\prime} \mathbf{2}$ in Figure S18 and Figure S19.

\begin{tabular}{cccc}
\hline Entry & $\boldsymbol{A G}_{\boldsymbol{B} 3^{a}}$ & $\mathbf{E}_{\mathbf{B} 3}{ }^{a}$ & $\mathbf{G}_{\mathrm{B3}}{ }^{a}$ \\
\hline P'1-TS5 & -3.6 & -2808.283881 & -2807.726895 \\
P'1-IN5 & -14.4 & -2808.307626 & -2807.744062 \\
P'1-TS6 & $\mathbf{1 7 . 6}$ & -2808.257889 & -2807.692985 \\
P'2-TS5 & $\mathbf{4 . 4}$ & -2808.272256 & -2807.714037 \\
P'2-IN5 & $-\mathbf{6 . 0}$ & -2808.292531 & -2807.730597 \\
P'2-TS6 & $\mathbf{1 8 . 4}$ & -2808.25453 & -2807.691733 \\
\hline
\end{tabular}

${ }^{a} \mathbf{E}_{\mathbf{B} 3}, \mathbf{G B}_{\mathbf{B} 3}$,and $\boldsymbol{\Delta} \boldsymbol{G}_{\boldsymbol{B} 3}$ are obtained at B3LYP-D3/6-31+G*/(SMD, solvent = dichloromethane) level. 


\section{Calculations on the regioselective Scholl reaction from 4 to 3 (Figure}

\section{S20-S23)}
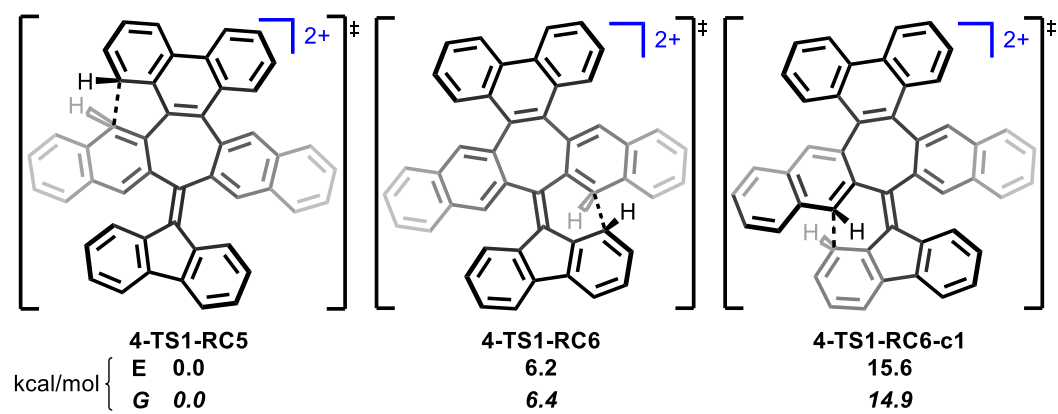

Figure S20. Relative electronic energies $(\Delta \mathrm{E})$ and free energies $(\Delta G)$ for the first electrocyclization of $4^{2+}$ via 5 -membered ring cyclization vs. the 6-membered ring cyclization. Energy unit: kcal/mol. Calculated at B3LYP-D3/6-31+G(d) in solution $($ SMD model, solvent $=$ dichloromethane $)$.

The high regioselectivity of syn- double cyclizations of compound $\mathbf{4}$ to $\mathbf{3}$ was studied via DFT calculations. Preliminary studies on dication electrocyclization of compound 4 suggest that, 5-membered ring electrocyclization at phenanthryl subunit is highly favored over the 6-membered ring electrocyclization at fluorenyl subunit with an enegy differene of $6.4 \mathrm{kcal} / \mathrm{mol}$ (Figure S20).
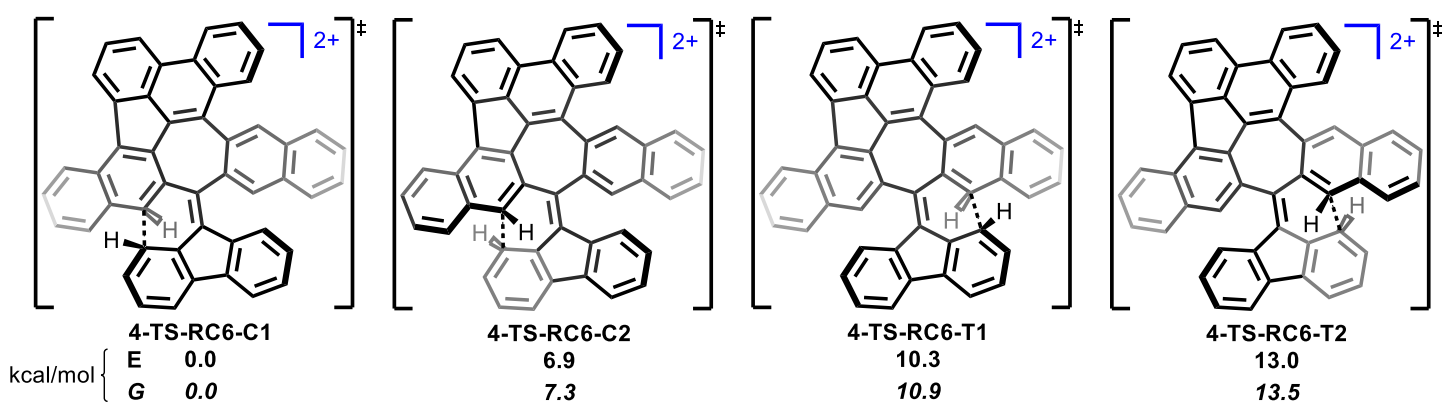

Figure S21. Relative electronic energies $(\Delta \mathrm{E})$ and free energies $(\Delta G)$ for the second electrocyclization via 6-membered ring cyclizations at the fluorenyl subunit. Calculated at B3LYP-D3/6-31+G(d) in solution (SMD model, solvent = dichloromethane).

On the second electrocyclization, syn- electrocyclizations are always favored with significant free energy advantages of at least $3.6 \mathrm{kcal} / \mathrm{mol}$ over the anti- cyclization (Figure S21). 


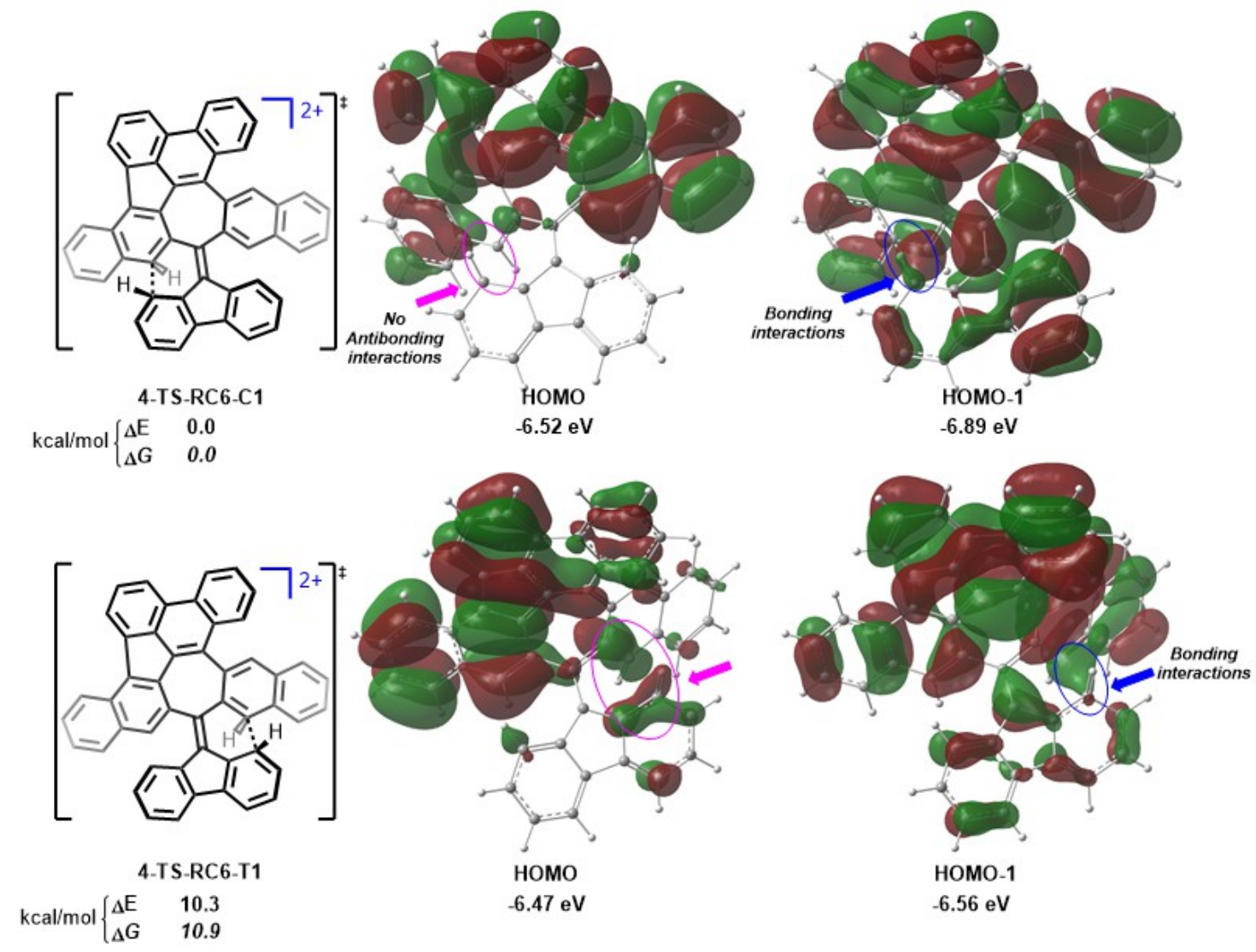

Figure S22. Bonding and/or antibonding interactions in selected molecular orbitals of 4-TS-RC6-C1 and 4-TS-RC6-T1. Calculated at B3LYP-D3/6-31+G(d) in solution (SMD model, solvent $=$ dichloromethane $)$. Isoval = 0.015.

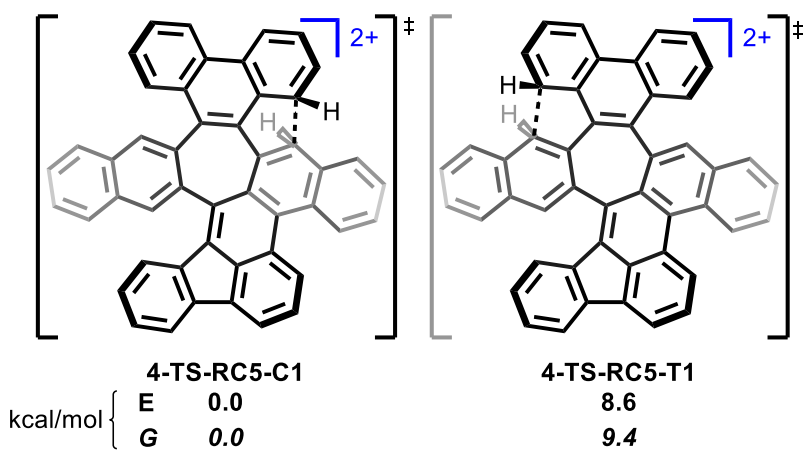

Figure S23. Relative electronic energies $(\Delta \mathrm{E})$ and free energies $(\Delta G)$ for the second electrocyclization via 5-membered ring cyclization at the phenanthryl subunit. Calculated at B3LYP-D3/6-31+G(d) in solution (SMD model, solvent = dichloromethane). 


\section{Calculations for the regioselective Scholl reaction from 2 to 5 (Table S10,}

\section{Figure S24)}

In the Scholl reaction from compound $\mathbf{2}$ to $\mathbf{5}$, the regioselectivity preference for $\mathbf{5}$ over $\mathbf{6}$ was attributed to dication electrocyclization mechanism. ${ }^{[3]}$ As shown in Table S10, 4 different transition conformations were considered for anti- and syn- cyclizations respectively. DFT calculations suggest that anti- cyclization to $\mathbf{5}$ is favored, and the regioselectivity for $\mathbf{5}$ vs. $\mathbf{6}$ is about 4.17:1, which is in quanlitatively good agreement with the experimental result (68\%:25\%, approximately $2.72: 1)$.

Table S10. Relative free energies/ratios for dication electrocyclization transition states through anti- $(T)$ or syn- $(C)$ cyclizations, and regioselectivity based on this dication electrocyclization mechanism. Please refer to Figure S24 for transition state geometries. Calculated at B3LYP-D3/6-31+G(d) in solution (SMD model, solvent = dichloroethane).

\begin{tabular}{|c|c|c|c|c|c|c|}
\hline Entry & $\begin{array}{c}\Delta G \\
\text { (kcal/mol) }\end{array}$ & Relative Ratio & $\begin{array}{r}\text { Perce } \\
(\% \\
\end{array}$ & ) & Regioselectivity & Product \\
\hline 2-TS2-T1 & 0.0 & 1.00 & 54.47 & \multirow{4}{*}{$80.65 \%$} & \multirow{4}{*}{4.17} & \multirow{4}{*}{5} \\
\hline 2-TS2-T2 & 0.4 & 0.48 & 26.17 & & & \\
\hline 2-TS2-T3 & 5.4 & $1.03 * 10^{-4}$ & $5.6 * 10^{-3}$ & & & \\
\hline 2-TS2-T4 & 6.9 & $8.55^{*} 10^{-6}$ & $4.7 * 10^{-4}$ & & & \\
\hline 2-TS2-C1 & 2.7 & $9.95 * 10^{-3}$ & 0.54 & \multirow{4}{*}{$19.35 \%$} & \multirow{4}{*}{1.00} & \multirow{4}{*}{6} \\
\hline 2-TS2-C2 & 1.3 & 0.11 & 6.16 & & & \\
\hline 2-TS2-C3 & 1.9 & $3.75 * 10^{-2}$ & 2.04 & & & \\
\hline 2-TS2-C4 & 1.0 & 0.20 & 10.60 & & & \\
\hline
\end{tabular}
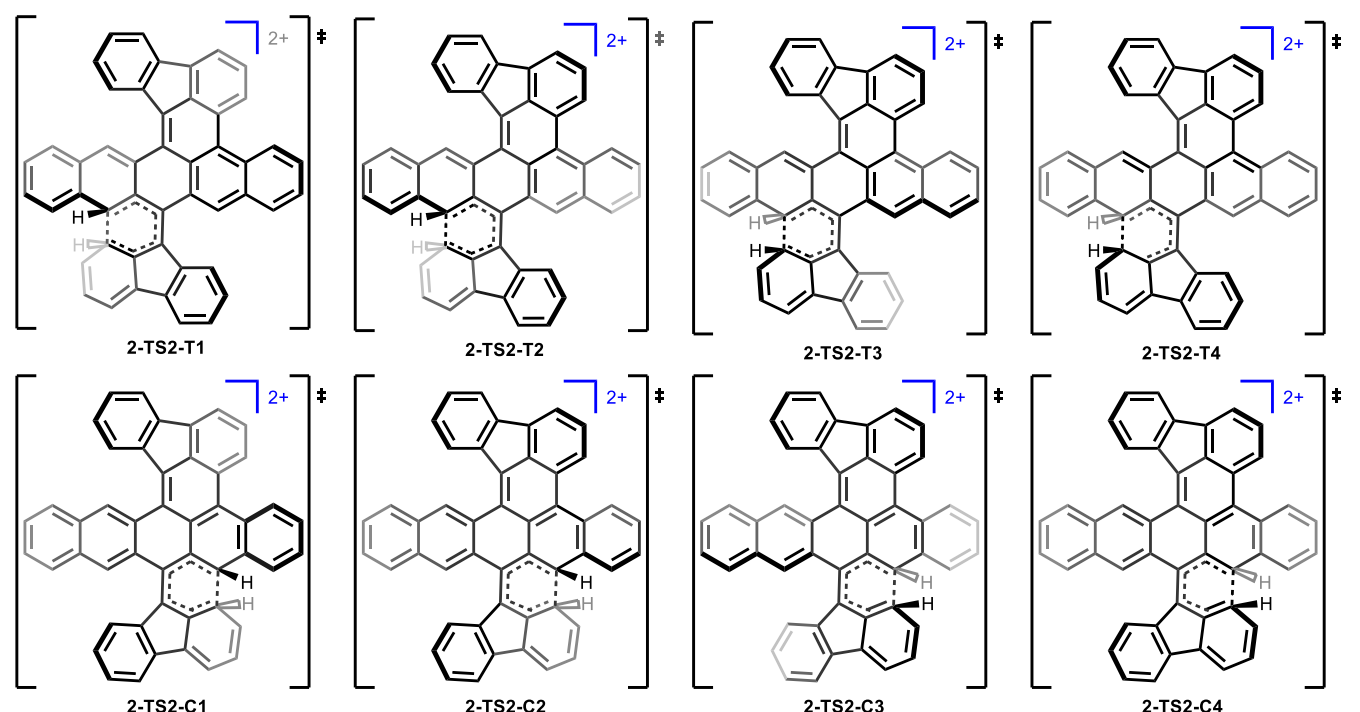

Figure S24. Different conformations of the second electrocyclization transition states through anti- $(T)$ or syn- $(C)$ cyclizations for compound 2. Please refer to Table S10 for relative free energies/ratios. 


\section{Calculations for the regioselective Mallory reaction from 2 to 6 (Figure S25,}

\section{Table S11)}

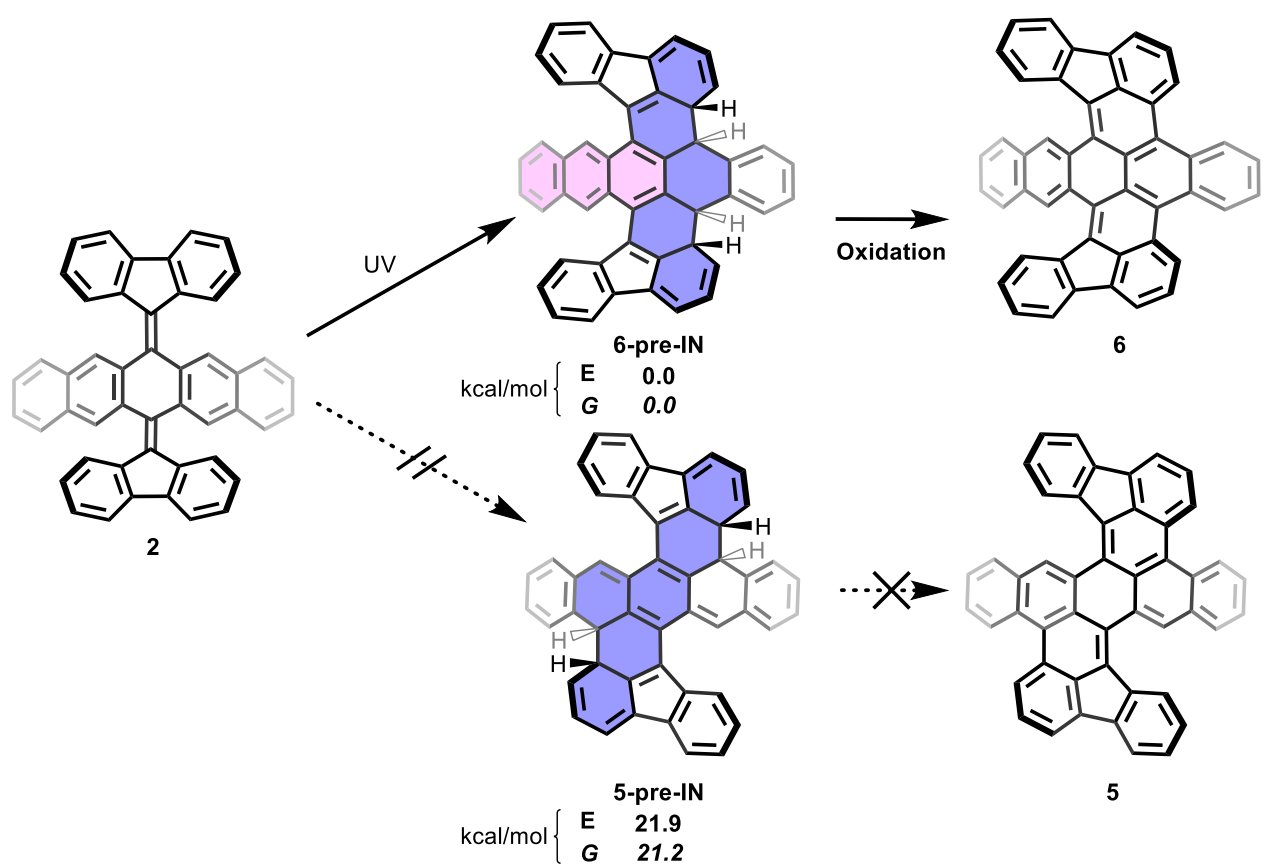

Figure S25. Relative energies for syn- and anti-cyclization intermediates formed via UV irradiation induced double photocyclization. Calculated at B3LYP-D3/6-31+G(d) level in gas phase.

According to DFT calculations in Figure S25, under Mallory photocyclization condition, the syncyclization oxidation intermediate 6-pre-IN is thermodynamically favored over the anti-cyclization oxidation intermediate 5-pre-IN by merely $21.2 \mathrm{kcal} / \mathrm{mol}$, suggesting that $s y n$ - cyclization oxidation pathway to 6 is favored. There are 2 dearomatized rings (blue, Figure S25) less in 6-pre-IN than those in 5-pre-IN. Besides, a newly formed anthracene fragment (purple) in 6-pre-IN obviously contributes more $\pi$-conjugation than the phenyl fragment in 5-pre-IN, in accordance with the Clar's sextet rule. ${ }^{[4]}$ 
Table S11. The absolute energy (electronic and Gibbs free energy, i.e. $\mathbf{E}$ and $\boldsymbol{G}$, unit: Hartree) for transition states in Figure S20-S25, Table S10.

\begin{tabular}{ccc}
\hline \multicolumn{1}{c}{ Entry } & $\mathbf{E}_{\mathbf{B 3}}$ & $\mathbf{G}_{\mathbf{B 3}}$ \\
\hline 4-TS1-RC5 $^{a}$ & -1845.795258 & -1845.255046 \\
4-TS1-RC6 $^{a}$ & -1845.785421 & -1845.244810 \\
4-TS1-RC6-c1 $^{a}$ & -1845.770393 & -1845.231306 \\
4-TS-RC6-C $^{a}$ & -1844.605139 & -1844.084873 \\
4-TS-RC6-C2 $^{a}$ & -1844.594150 & -1844.073205 \\
4-TS-RC6-T1 $^{a}$ & -1844.588721 & -1844.067468 \\
4-TS-RC6-T2 $^{a}$ & -1844.584413 & -1844.063380 \\
4-TS-RC5-C1 $^{a}$ & -1844.612593 & -1844.092920 \\
4-TS-RC5-T1 $^{a}$ & -1844.598957 & -1844.077927 \\
2-TS2-T1 $^{b}$ & -1844.604368 & -1844.083086 \\
2-TS2-T2 $^{b}$ & -1844.602959 & -1844.082394 \\
2-TS2-T3 $^{b}$ & -1844.595902 & -1844.074421 \\
2-TS2-T4 $^{b}$ & -1844.593225 & -1844.072068 \\
2-TS2-C1 $^{b}$ & -1844.601266 & -1844.078733 \\
2-TS2-C2 $^{b}$ & -1844.601884 & -1844.081028 \\
2-TS2-C3 $^{b}$ & -1844.600457 & -1844.079985 \\
${\text { 2-TS2- } \boldsymbol{C 4}^{b}}^{b}$ & -1844.602289 & -1844.081541 \\
5-pre-IN $^{c}$ & -1846.040682 & -1845.498705 \\
6-pre-IN $^{c}$ & -1846.075635 & -1845.532566 \\
\hline
\end{tabular}

Note: $\mathbf{E}_{\mathbf{B} 3}$ and $\mathbf{G}_{\mathbf{B} 3}$ are obtained at

${ }^{a}$ B3LYP-D3/6-31+G*/(SMD model, solvent = dichlormoethane $)$ level;

${ }^{b}$ B3LYP-D3/6-31+G*/(SMD model, solvent = dichloroethane) level;

${ }^{c}$ B3LYP-D3/6-31+G* level in gas phase. 


\section{Frontier molecular orbital plots for compounds 2-6 (Figure S26-S29)}
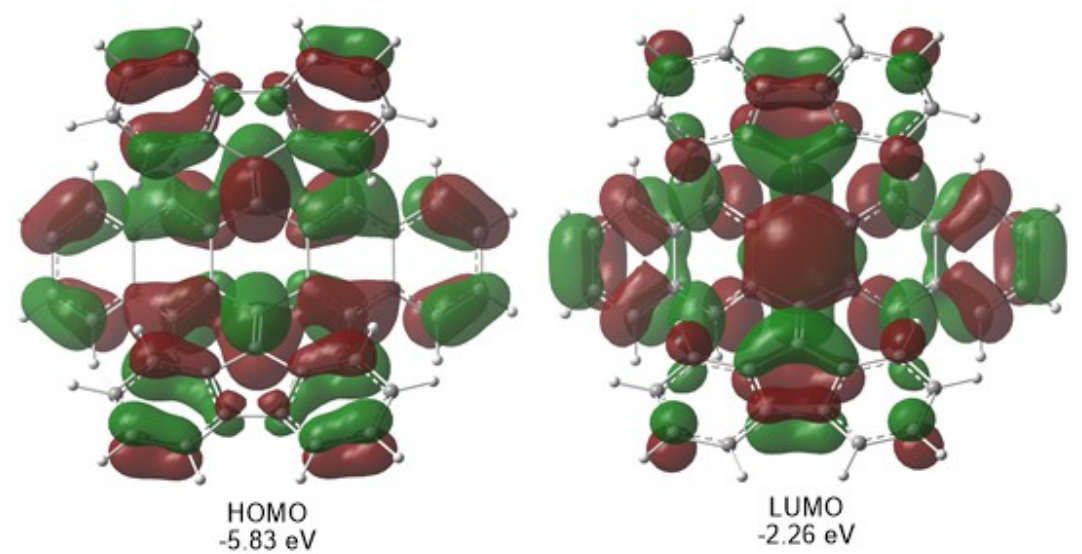

Figure S26. HOMO and LUMO of compound 2. Calculated at B3LYP-D3/6-311+G(2d,p) level in gas phase. Isoval $=0.02$.

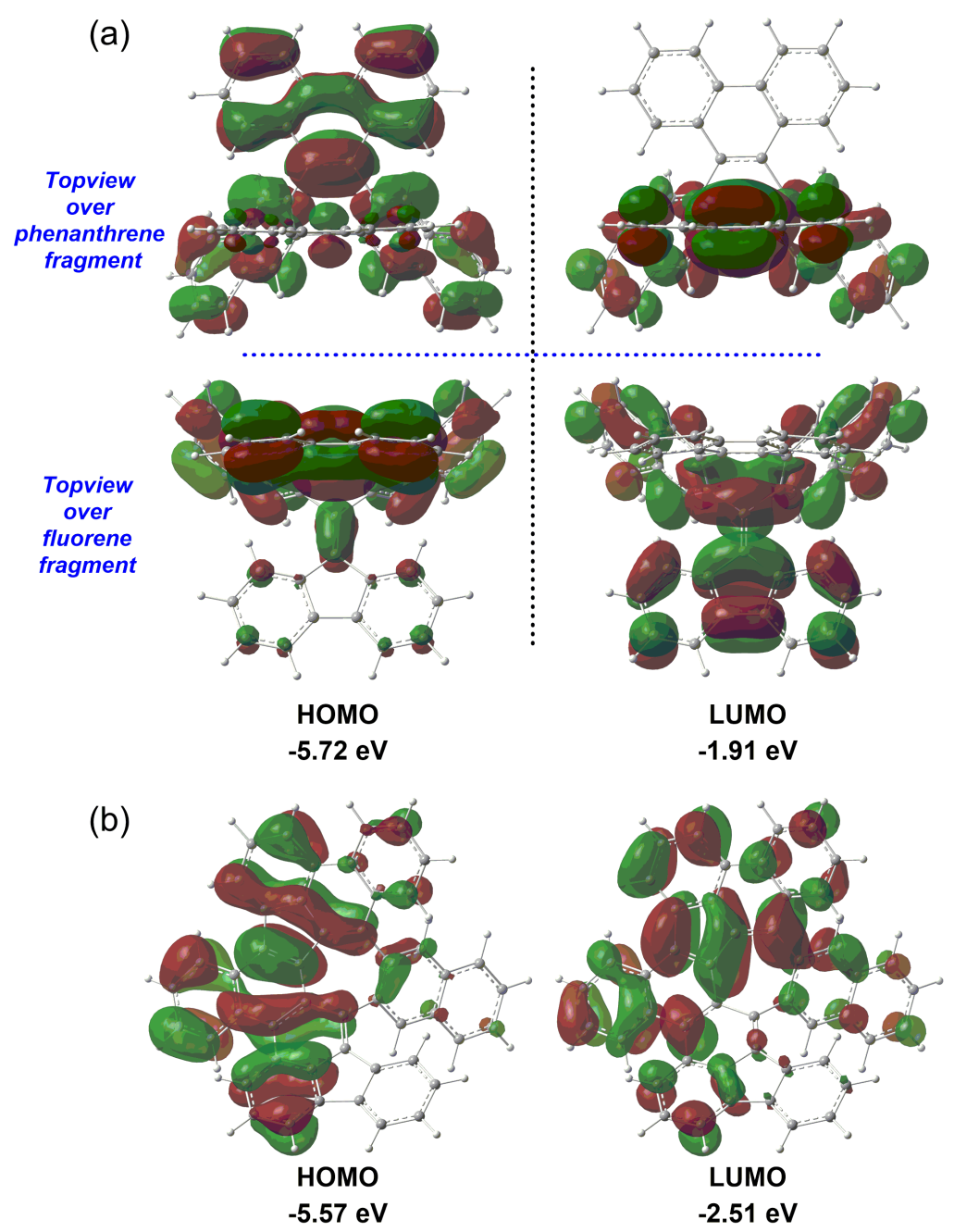

Figure 27. Frontier molecular orbitals and energies: (a) for $\mathbf{4}$ and (b) for 3. Calculated at B3LYP-D3/6-311+G(2d,p) level in gas phase. Isoval $=0.02$. 

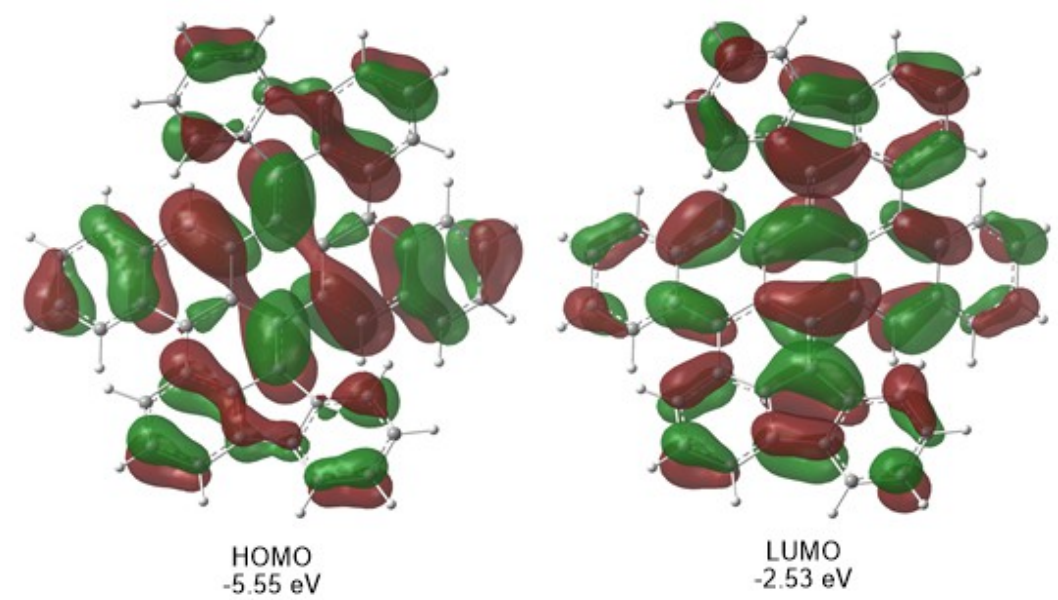

Figure S28. HOMO and LUMO of compound 5. Calculated at B3LYP-D3/6-311+G(2d,p) level in gas phase. Isoval $=0.02$.
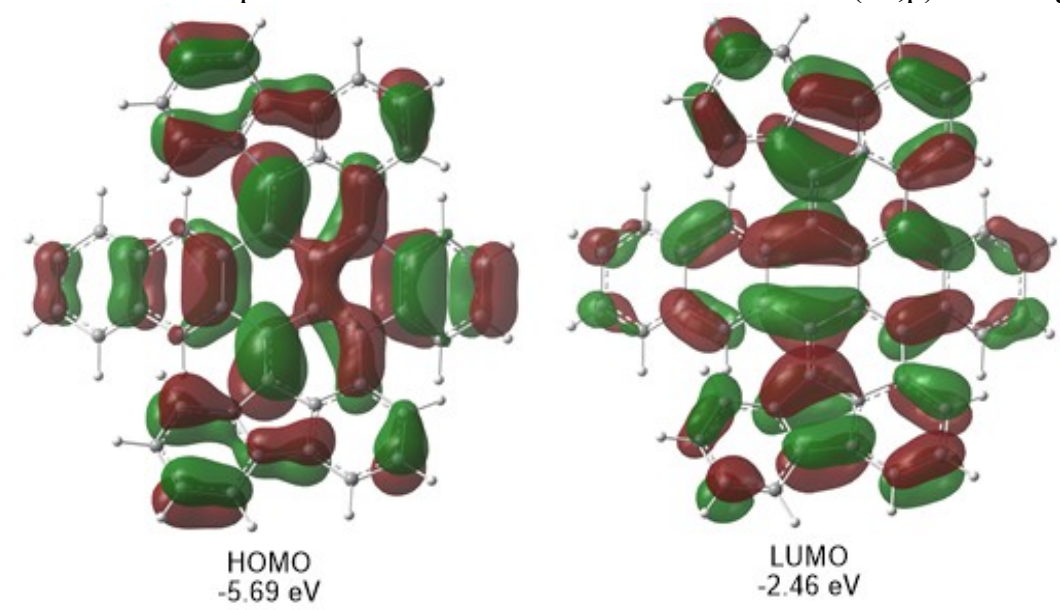

Figure S29. HOMO and LUMO of compound 6. Calculated at B3LYP-D3/6-311+G(2d,p)level in gas phase. Isoval = 0.02 . 


\section{Exclusion of other possible cyclizaion mechanisms and other rearrangement under TfOH/DDQ condition with the 2 nd cyclization from 4 to 3 as computational model (Figure S30-S37, Table S12)}

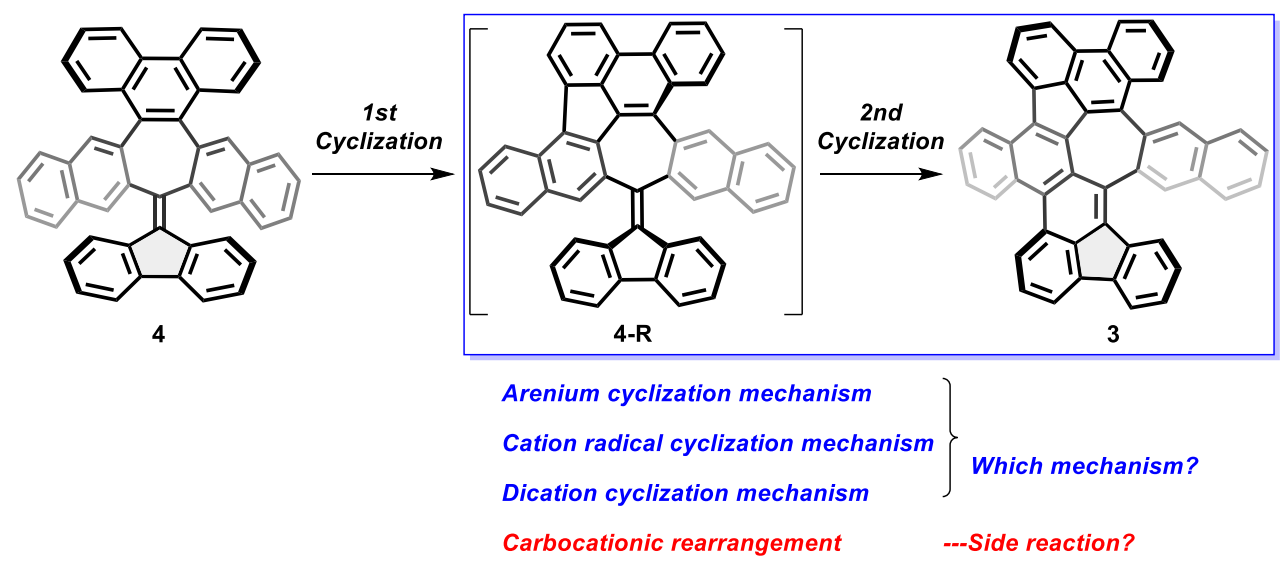

Figure S30. Three possible cyclization mechanisms and possible rearrangements were investigated by DFT calculations.

For Scholl reaction, three possible mechanisms in general (arenium-ion mechanism, ${ }^{[5]}$ cation-radical mechanism ${ }^{[6]}$ and dication mechanism ${ }^{[7]}$ ) were proposed for Scholl reaction in the literature. To figure out which of the above three mechanisms dominates the reaction from 4 to 3 , three possible cyclization mechanisms (i.e., arenium-ion cyclization, cation-radical cyclization and dication cyclization mechanism) and carbocationic rearrangments were investigated as shown in Figure S30. All of the above three mechanisms are stepwise. As shown in Figure S20, the calculation results of dication cyclization mechanism from $\mathbf{4}$ to $\mathbf{3}$ suggest that the first cyclization at phenanthryl subunit is favored. Based on this conclusion, the first cyclization product intermediate $\mathbf{4 - R}$ was chosen as the computational model to investigate that which of the above three mechanisms was more reasonable for 4-R to 4 under TfOH/DDQ. (Figure S30) Also, as shown in Scheme 1 and Figure 2 of the main text, with $\mathrm{TfOH}$, carbocationic rearrangement for $\mathbf{4}-\mathbf{R}$ is also possible. 


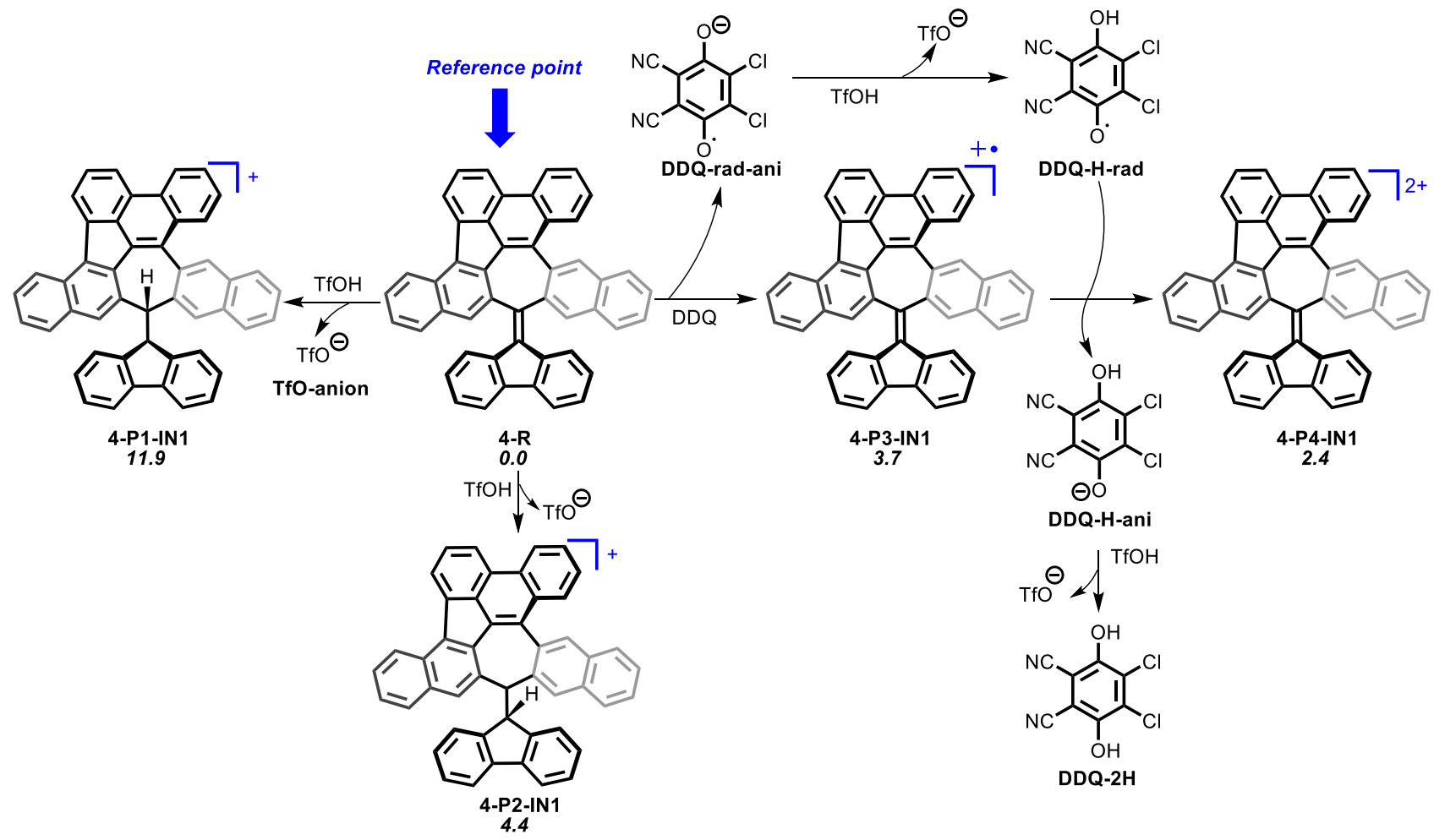

Figure S31. Relative free energies $(\Delta G$, unit: $\mathrm{kcal} / \mathrm{mol})$ of protonation, oxidation intermediates (by losing one electron /two electrons) from the first cyclization product 4-R under TfOH/DDQ condition. Calculated at B3LYP-D3/6-31+G*/(SMD, solvent=dichloromethane) level.

First of all, protonation, oxidation intermediates (by losing one electron /two electrons) from the first cyclization product 4-R with TfOH/DDQ were calculated. As shown in Figure S31, protanation, one-electron/two-electron oxidation are thermodynamically possible under TfOH/DDQ at ambient temperature. ${ }^{[8]}$ As shown in Figure S32-S37, the dicationic cyclization (4-TS-RC6-C1, $14.5 \mathrm{kcal} / \mathrm{mol}$, Figure S35) at syn-position is more favorable than the other two (Figure S32-S34).
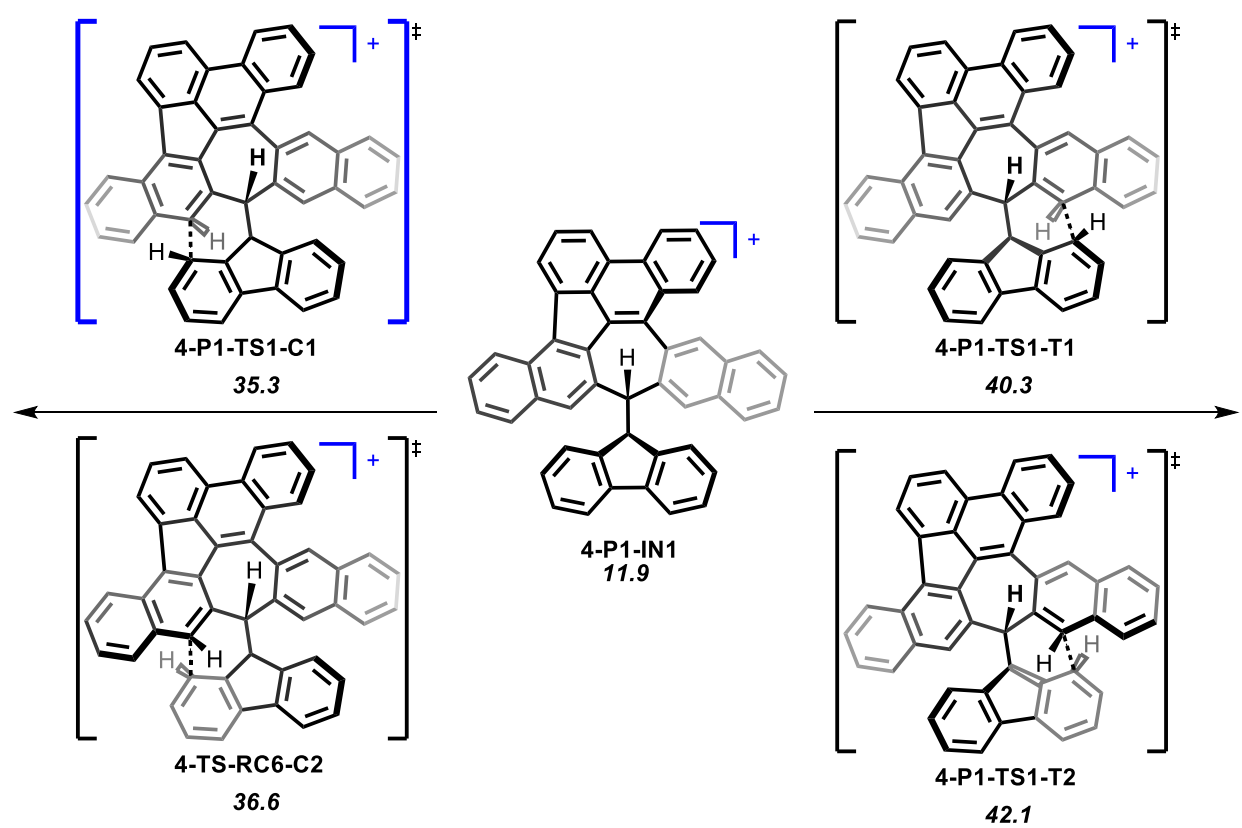

Figure S32. Relative free energies $(\Delta G$, unit: $\mathrm{kcal} / \mathrm{mol})$ of the cyclization transition states via arenium-ion mechanism for protonation product at the central 7-memebered ring. Calculated at B3LYP-D3/6-31+G*/(SMD, solvent = dichloromethane). 

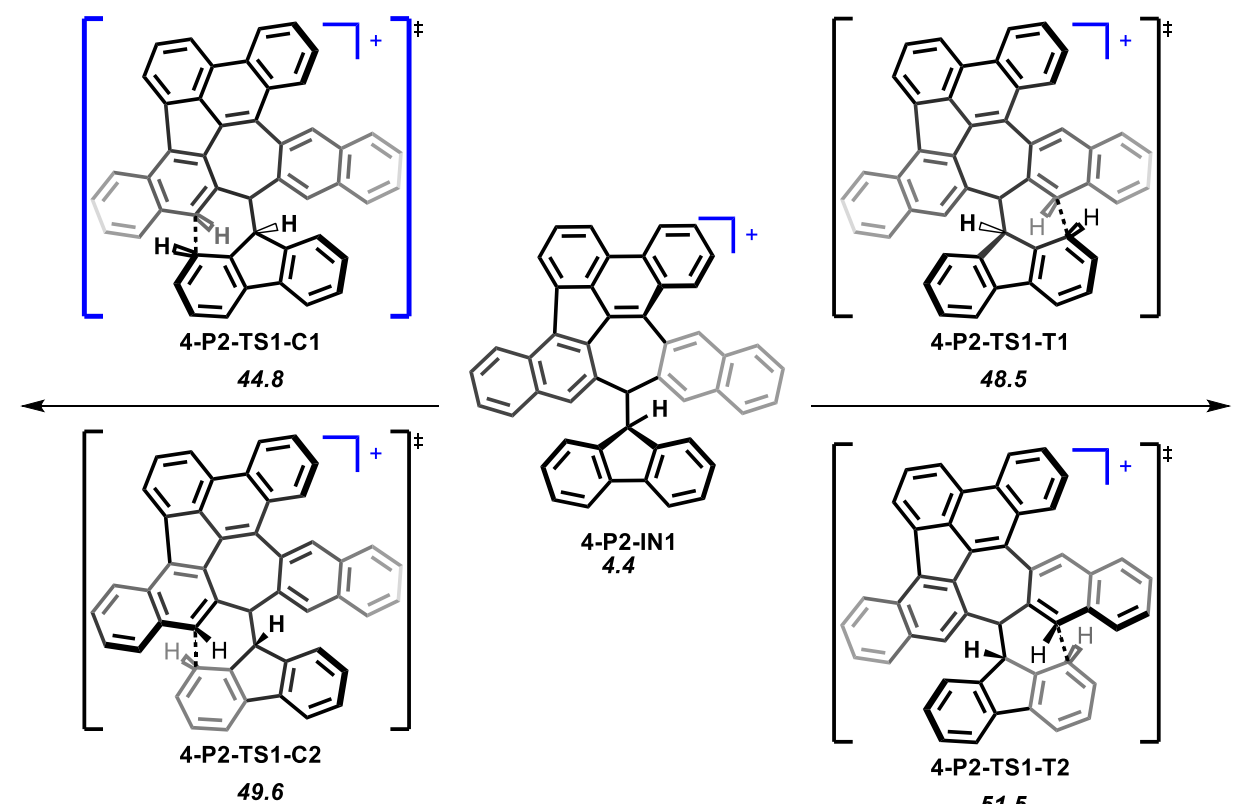

Figure S33. Relative free energies $(\Delta G$, unit: $\mathrm{kcal} / \mathrm{mol})$ of the cyclization transition states via arenium-ion mechanism for protonation product at fluorenyl carbon. Calculated at B3LYP-D3/6-31+G*/(SMD, solvent = dichloromethane).
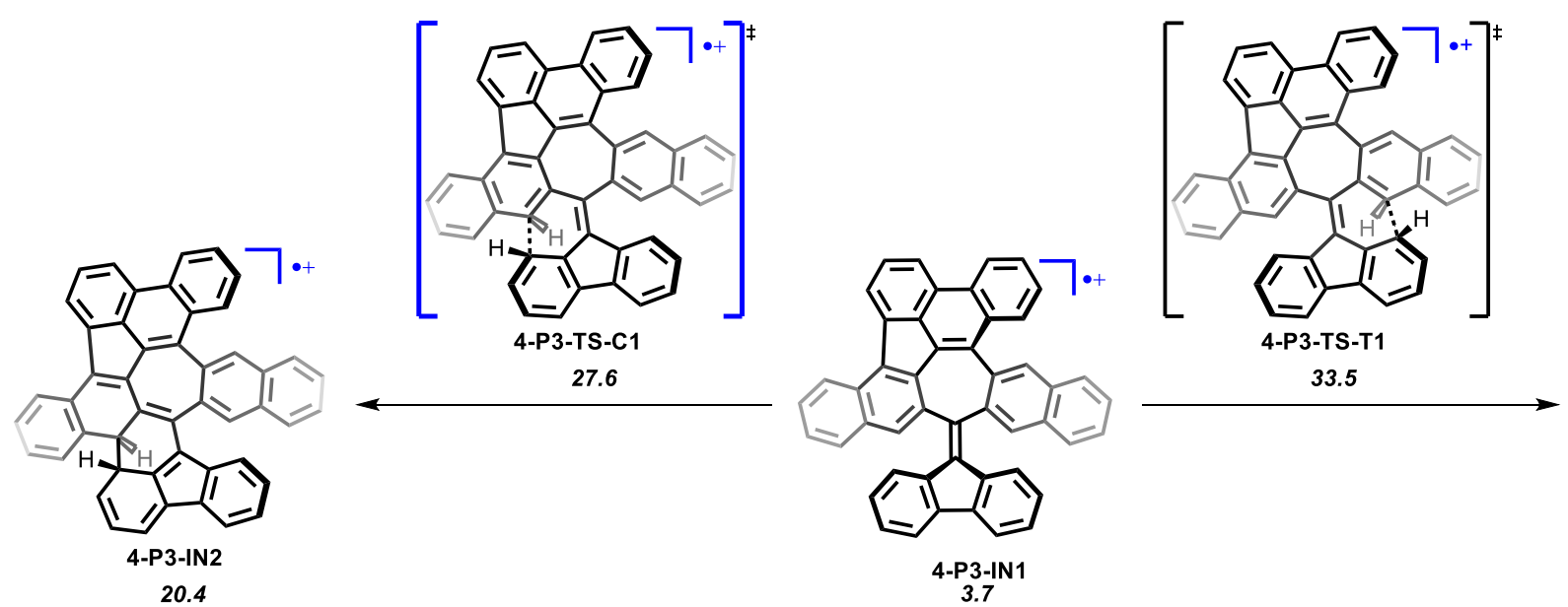

Figure S34. Relative free energies ( $\triangle G$, unit: $\mathrm{kcal} / \mathrm{mol})$ of the cyclization transition states via cation-radical mechanism for one-electron oxidation product at fluorenyl carbon. Calculated at B3LYP-D3/6-31+G*/(SMD, solvent = dichloromethane). 

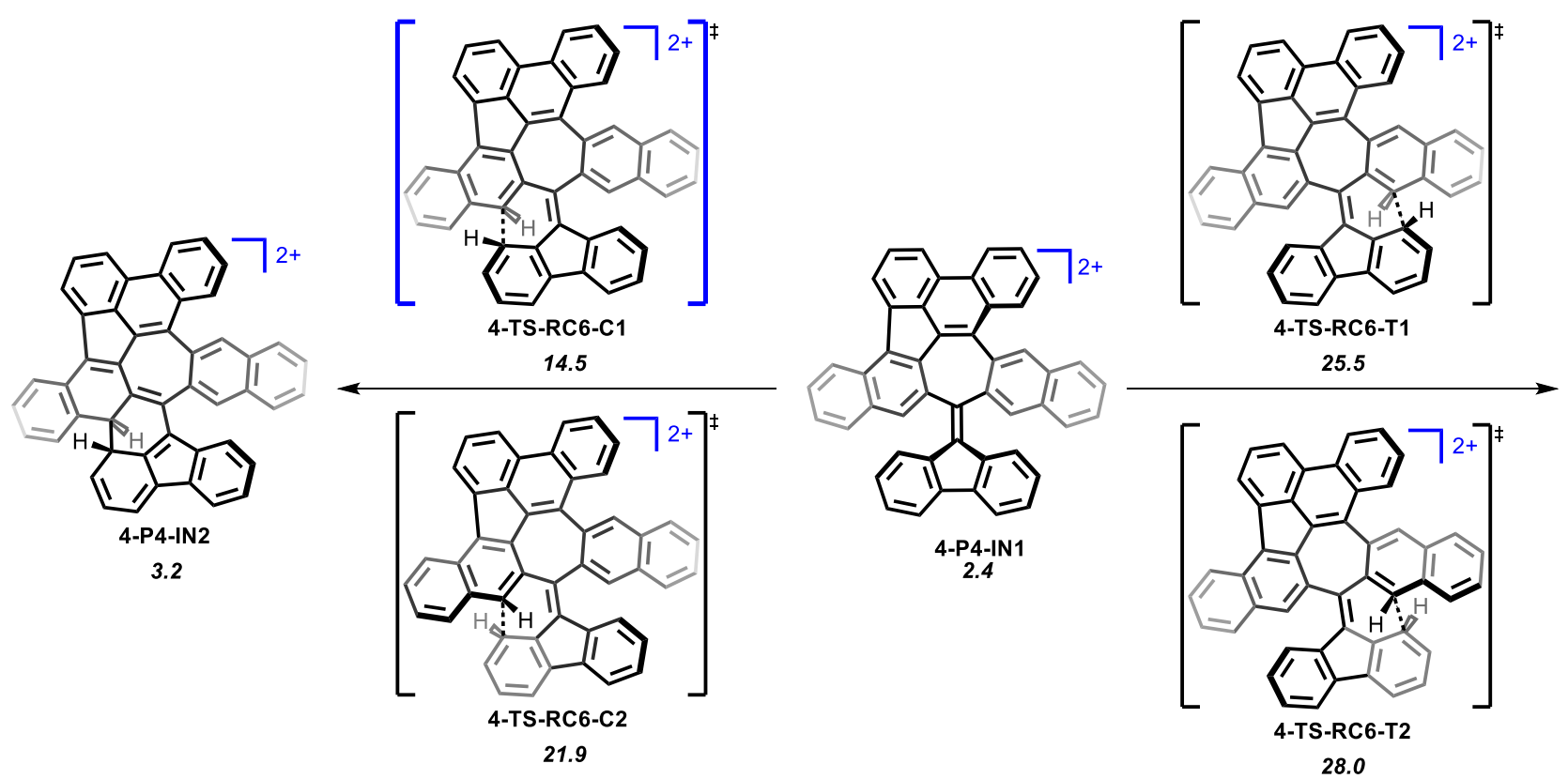

Figure S35. Relative free energies $(\Delta G$, unit: $\mathrm{kcal} / \mathrm{mol})$ of the cyclization transition states via dication mechanism for two-electron oxidation product at fluorenyl carbon. Calculated at B3LYP-D3/6-31+G*/(SMD, solvent = dichloromethane).

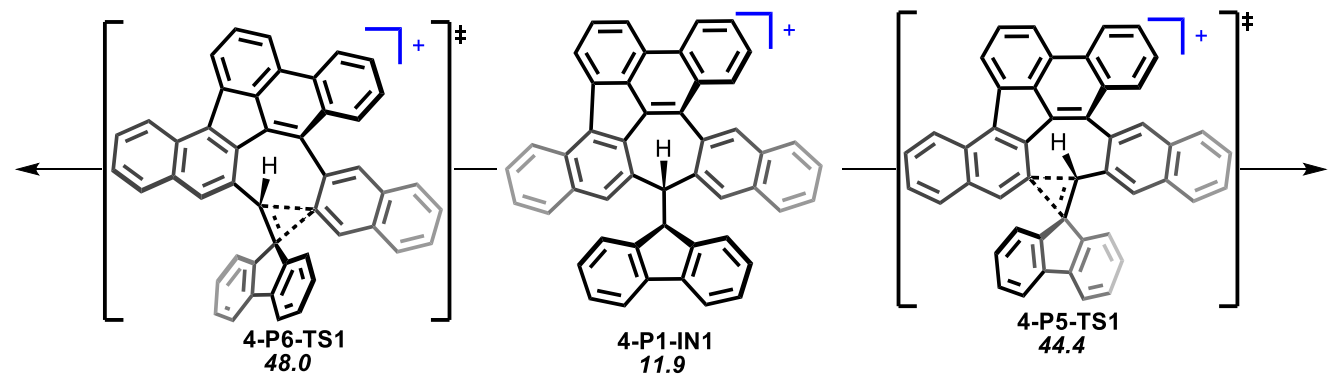

Figure S36. Relative free energies $(\Delta G$, unit: $\mathrm{kcal} / \mathrm{mol})$ of the carbocationic rearrangement transition for protonation intermediate at heptagonal carbon. Calculated at B3LYP-D3/6-31+G*/(SMD, solvent = dichloromethane).

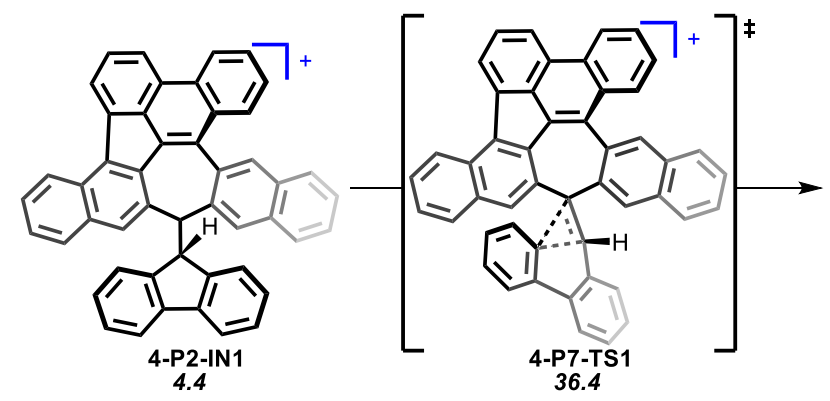

Figure S37. Relative free energies of the carbocationic rearrangement transition for protonation intermediate at fluorenyl carbon. Calculated at B3LYP-D3/6-31+G*/(SMD, solvent = dichloromethane). 
Table S12. The absolute energy (electronic and Gibbs free energy, i.e. E and G, unit: Hartree) for intermediates and transition states in Figure S30-S36.

\begin{tabular}{ccc}
\hline Entry & $\mathbf{E}_{\mathbf{B 3}}{ }^{a}$ & $\mathbf{G}_{\mathbf{B 3}}{ }^{a}$ \\
\hline 4-R & -1845.034808 & -1844.517455 \\
DDQ & -1485.149664 & -1485.126954 \\
TfO-anion & -961.629847 & -961.636072 \\
DDQ-rad-ani & -1485.335300 & -1485.314274 \\
DDQ-H-rad & -1485.762353 & -1485.729578 \\
DDQ-H-ani & -1485.945307 & -1485.912236 \\
DDQ-2H & -1486.399674 & -1486.354616 \\
4-P1-IN1 & -1845.438416 & -1844.907533 \\
4-P1-TS1-T1 & -1845.394255 & -1844.862335 \\
4-P1-TS1-C1 & -1845.403351 & -1844.870381 \\
4-P1-TS1-T2 & -1845.391437 & -1844.859551 \\
4-P1-TS1-C2 & -1845.400109 & -1844.868232 \\
4-P5-TS1 & -1845.385271 & -1844.855768 \\
4-P6-TS1 & -1845.380219 & -1844.850108 \\
4-P2-IN1 & -1845.449655 & -1844.919500 \\
4-P2-TS1-T2 & -1845.375761 & -1844.844447 \\
4-P2-TS1-C1 & -1845.385878 & -1844.855222 \\
4-P2-TS1-C2 & -1845.379248 & -1844.847507 \\
4-P2-TS1-T1 & -1845.380203 & -1844.849237 \\
4-P7-TS1 & -1845.398072 & -1844.868593 \\
4-P3-IN1 & -1844.834292 & -1844.317988 \\
4-P3-TS-T1 & -1844.789019 & -1844.270585 \\
4-P3-TS-C1 & -1844.798983 & -1844.279891 \\
4-P3-IN2 & -1844.812811 & -1844.291483 \\
4-P4-IN1 & -1844.623688 & -1844.104199 \\
4-P4-IN2 & -1844.626046 & -1844.102916 \\
\hline & & \\
\hline
\end{tabular}

${ }^{a} \mathbf{E}_{\mathbf{B} 3}, \mathbf{G}_{\mathbf{B} 3}$, are obtained at B3LYP-D3/6-31+G*/(SMD model, solvent = dichloromethane $)$. 


\section{TD-DFT Calculations of compounds 2-6 (Table S13-S18)}

Table S13. TD-DFT calculation of compound 2 at B3LYP-D3/6-311+G(2d,p) level in gas phase. (HOMO=158, LUMO=159).

\begin{tabular}{|c|c|c|c|c|}
\hline Excited state & Energy (eV) & Wavelength (nm) & Oscillator strength (f) & $\begin{array}{c}\text { Description } \\
\end{array}$ \\
\hline 1 & 3.0454 & 407.12 & 0.0393 & $\begin{array}{lc}\text { HOMO-1 -> LUMO } & 0.35106 \\
\text { HOMO -> LUMO+1 } & 0.60922 \\
\end{array}$ \\
\hline 2 & 3.0730 & 403.46 & 0.0388 & $\begin{array}{ll}\text { HOMO ->LUMO } & 0.70445\end{array}$ \\
\hline 3 & 3.1120 & 398.41 & 0.0000 & $\begin{array}{ll}\text { HOMO-3 -> LUMO+1 } & 0.52280 \\
\text { HOMO-2 -> LUMO } & -0.46657 \\
\end{array}$ \\
\hline 4 & 3.1135 & 398.21 & 0.0006 & $\begin{array}{lc}\text { HOMO-3 -> LUMO } & -0.46349 \\
\text { HOMO-2 -> LUMO+1 } & 0.52586\end{array}$ \\
\hline 5 & 3.2198 & 385.06 & 0.2969 & HOMO-1 -> LUMO+1 0.70323 \\
\hline 6 & 3.2717 & 378.95 & 0.3795 & $\begin{array}{lcl}\text { HOMO-1 }->\text { LUMO } & 0.60747 \\
\text { HOMO -> } \quad \text { LUMO+1 } & -0.34947\end{array}$ \\
\hline 7 & 3.3463 & 370.51 & 0.0480 & $\begin{array}{l}\text { HOMO-5 -> LUMO } 0.16376 \\
\text { HOMO-4 -> LUMO+1 } 0.66667\end{array}$ \\
\hline 8 & 3.3710 & 367.80 & 0.0000 & $\begin{array}{l}\text { HOMO-4 -> LUMO } 0.10805 \\
\text { HOMO-3 -> LUMO+1 } 0.46051 \\
\text { HOMO-2 -> LUMO } 0.52097\end{array}$ \\
\hline 9 & 3.3723 & 367.66 & 0.0039 & $\begin{array}{lc}\text { HOMO-4 -> LUMO+1 } & 0.12261 \\
\text { HOMO-3 -> LUMO } & 0.52076 \\
\text { HOMO-2 -> LUMO+1 } & 0.45650\end{array}$ \\
\hline 10 & 3.3830 & 366.49 & 0.0000 & $\begin{array}{l}\text { HOMO-5 -> LUMO+1 } 0.12269 \\
\text { HOMO-4 -> LUMO } 0.68113\end{array}$ \\
\hline
\end{tabular}


Table S14. TD-DFT calculation of compound 3 at B3LYP-D3/6-311+G(2d,p)//B3LYP-D3/6-311+G(2d,p) level in gas phase. (HOMO=158, LUMO=159).

\begin{tabular}{|c|c|c|c|c|}
\hline Excited state & Energy (eV) & Wavelength (nm) & Oscillator strength (f) & Description \\
\hline 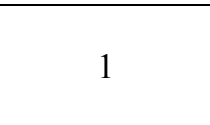 & 2.5623 & 483.89 & 0.0588 & $\begin{array}{lc}\text { HOMO-2 -> LUMO } & -0.12508 \\
\text { HOMO-1 -> LUMO } & 0.21425 \\
\text { HOMO -> LUMO } & 0.64043\end{array}$ \\
\hline 2 & 2.6711 & 464.17 & 0.0372 & $\begin{array}{lc}\text { HOMO-2 -> LUMO } & 0.14506 \\
\text { HOMO-1 -> LUMO } & 0.53125 \\
\text { HOMO -> LUMO } & -0.18867 \\
\text { HOMO -> LUMO+1 } & -0.37836 \\
\end{array}$ \\
\hline 3 & 2.8910 & 428.86 & 0.1324 & $\begin{array}{lc}\text { HOMO-2 -> LUMO } & 0.23001 \\
\text { HOMO-1 -> LUMO } & 0.31997 \\
\text { HOMO-1 -> LUMO+1 } & -0.11526 \\
\text { HOMO -> LUMO+1 } & 0.54837\end{array}$ \\
\hline 4 & 2.9264 & 423.67 & 0.1611 & $\begin{array}{lc}\text { HOMO-2 -> LUMO } & 0.59604 \\
\text { HOMO-1 -> LUMO } & -0.21349 \\
\text { HOMO-1 -> LUMO+1 } & -0.15451 \\
\text { HOMO -> LUMO } & 0.17904 \\
\text { HOMO -> LUMO+1 } & -0.13741 \\
\end{array}$ \\
\hline 5 & 3.0194 & 410.63 & 0.0842 & 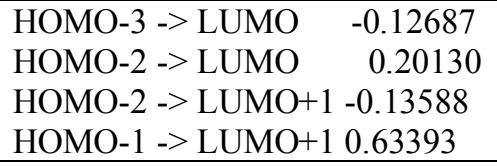 \\
\hline 6 & 3.1649 & 391.74 & 0.0418 & $\begin{array}{lc}\text { HOMO-3 -> LUMO } & 0.57290 \\
\text { HOMO-3 -> LUMO+1 } & 0.31012 \\
\text { HOMO-2 -> LUMO+1 } & 0.13103 \\
\text { HOMO-1 -> LUMO+1 } & 0.16447 \\
\text { HOMO -> LUMO+1 } & 0.10545 \\
\end{array}$ \\
\hline 7 & 3.2242 & 384.55 & 0.0978 & $\begin{array}{lr}\text { HOMO-3 -> LUMO } & -0.25338 \\
\text { HOMO-3 -> LUMO+1 } & 0.15589 \\
\text { HOMO-2 -> LUMO+1 } & 0.61115 \\
\end{array}$ \\
\hline 8 & 3.2777 & 378.27 & 0.0367 & $\begin{array}{lc}\text { HOMO-3 -> LUMO } & -0.26567 \\
\text { HOMO-3 -> LUMO+1 } & 0.58994 \\
\text { HOMO-2 -> LUMO+1 } & -0.22619 \\
\end{array}$ \\
\hline 9 & 3.3481 & 370.31 & 0.0093 & $\begin{array}{lr}\text { HOMO-4 -> LUMO } & 0.60006 \\
\text { HOMO-1 -> LUMO+2 } & 0.12529 \\
\text { HOMO -> LUMO+2 } & -0.24899 \\
\end{array}$ \\
\hline 10 & 3.4559 & 358.76 & 0.0725 & $\begin{array}{lc}\text { HOMO-4 -> LUMO } & 0.16370 \\
\text { HOMO-1 -> LUMO+2 } & 0.27856 \\
\text { HOMO -> LUMO+2 } & 0.52077 \\
\text { HOMO -> LUMO+3 } & -0.28768 \\
\end{array}$ \\
\hline
\end{tabular}


Table S15. TD-DFT calculation of compound 4 at B3LYP-D3/6-311+G(2d,p) level in gas phase. (HOMO=158, LUMO=159)

\begin{tabular}{|c|c|c|c|c|}
\hline Excited state & Energy (eV) & Wavelength (nm) & Oscillator strength (f) & Description \\
\hline 1 & 3.2588 & 380.46 & 0.0142 & HOMO -> LUMO \\
\hline 2 & 3.4018 & 364.46 & 0.0008 & 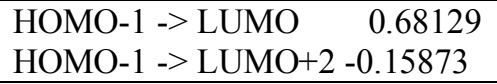 \\
\hline 3 & 3.5267 & 351.56 & 0.0126 & $\begin{array}{lc}\text { HOMO-3 -> LUMO } & -0.12308 \\
\text { HOMO -> LUMO+2 } & 0.68000 \\
\end{array}$ \\
\hline 4 & 3.5310 & 351.13 & 0.1070 & $\begin{array}{lc}\text { HOMO-4 -> LUMO+3 } & -0.10266 \\
\text { HOMO-2 -> LUMO } & -0.14697 \\
\text { HOMO -> LUMO+1 } & 0.66718 \\
\end{array}$ \\
\hline 5 & 3.6149 & 342.98 & 0.0590 & $\begin{array}{lc}\text { HOMO-3 -> LUMO+1 } & -0.11467 \\
\text { HOMO-2 -> LUMO } & 0.65055 \\
\text { HOMO-2 -> LUMO+2 } & 0.14374 \\
\text { HOMO -> LUMO+1 } & 0.15475 \\
\end{array}$ \\
\hline 6 & 3.6922 & 335.80 & 0.1398 & $\begin{array}{lc}\text { HOMO-4 -> LUMO+1 } & 0.17432 \\
\text { HOMO-3 -> LUMO } & 0.57581 \\
\text { HOMO-3 -> LUMO+2 } & 0.19760 \\
\text { HOMO -> LUMO+3 } & 0.27351 \\
\end{array}$ \\
\hline 7 & 3.7195 & 333.34 & 0.0435 & $\begin{array}{lc}\text { HOMO-4 -> LUMO+1 } & 0.30385 \\
\text { HOMO-3 -> LUMO } & -0.27058 \\
\text { HOMO-2 -> LUMO+1 } & -0.31020 \\
\text { HOMO -> LUMO+3 } & 0.43303 \\
\end{array}$ \\
\hline 8 & 3.8243 & 324.20 & 0.0004 & HOMO-1 -> LUMO+1 $\quad 0.70540$ \\
\hline 9 & 3.8284 & 323.85 & 0.0446 & $\begin{array}{ll}\text { HOMO-3 -> LUMO+1 } & 0.67493 \\
\text { HOMO-2 -> LUMO } & 0.11396\end{array}$ \\
\hline 10 & 3.8406 & 322.83 & 0.1038 & $\begin{array}{lc}\text { HOMO-5 -> LUMO } & 0.13584 \\
\text { HOMO-4 -> LUMO+1 } & 0.19534 \\
\text { HOMO-3 -> LUMO } & -0.17699 \\
\text { HOMO-3 -> LUMO+2 } & 0.10426 \\
\text { HOMO-2 -> LUMO+1 } & 0.57778 \\
\text { HOMO -> LUMO+2 } & -0.11532 \\
\text { HOMO -> LUMO+3 } & 0.16723\end{array}$ \\
\hline
\end{tabular}


Table S16. TD-DFT calculation of compound 5 at B3LYP-D3/6-311+G(2d,p) level in gas phase. (HOMO=158, $\mathrm{LUMO}=159$ ).

\begin{tabular}{|c|c|c|c|c|c|}
\hline Excited state & Energy (eV) & Wavelength (nm) & Oscillator strength (f) & \multicolumn{2}{|c|}{ Description } \\
\hline 1 & 2.6216 & 472.94 & 0.3677 & HOMO -> LUMO & 0.69494 \\
\hline 2 & 2.6428 & 469.14 & 0.0046 & $\begin{array}{l}\text { HOMO-1 -> LUMO } \\
\text { HOMO -> LUMO+1 }\end{array}$ & $\begin{array}{r}-0.39465 \\
0.57481\end{array}$ \\
\hline 3 & 2.9326 & 422.78 & 0.0710 & $\begin{array}{l}\text { HOMO-4 -> LUMO } \\
\text { HOMO-3 -> LUMO+1 } \\
\text { HOMO-2 -> LUMO } \\
\text { HOMO-1 -> LUMO+1 } \\
\text { HOMO -> LUMO+2 }\end{array}$ & $\begin{array}{c}-0.15208 \\
-0.11604 \\
0.54659 \\
0.20071 \\
0.32732\end{array}$ \\
\hline 4 & 2.9359 & 422.31 & 0.1447 & $\begin{array}{l}\text { HOMO-1 -> LUMO } \\
\text { HOMO -> LUMO+1 }\end{array}$ & $\begin{array}{c}0.57629 \\
0.38323 \\
\end{array}$ \\
\hline 5 & 2.9753 & 416.71 & 0.0044 & $\begin{array}{l}\text { HOMO-4 -> LUMO } \\
\text { HOMO-3 -> LUMO+1 } \\
\text { HOMO-2 -> LUMO } \\
\text { HOMO-1 -> LUMO+1 } \\
\text { HOMO -> LUMO+2 }\end{array}$ & $\begin{array}{c}-0.13475 \\
-0.12757 \\
-0.22906 \\
0.62796 \\
-0.10017\end{array}$ \\
\hline 6 & 3.0104 & 411.86 & 0.0061 & $\begin{array}{l}\text { HOMO-4 -> LUMO+1 } \\
\text { HOMO-3 -> LUMO }\end{array}$ & $\begin{array}{l}0.32648 \\
0.61315\end{array}$ \\
\hline 7 & 3.0209 & 410.42 & 0.0164 & $\begin{array}{l}\text { HOMO-4 -> LUMO } \\
\text { HOMO-3 -> LUMO+1 } \\
\text { HOMO-2 -> LUMO } \\
\text { HOMO-1 -> LUMO+1 }\end{array}$ & $\begin{array}{c}0.58573 \\
0.28343 \\
0.12256 \\
0.22797\end{array}$ \\
\hline 8 & 3.1620 & 392.11 & 0.0006 & $\begin{array}{l}\text { HOMO-4 -> LUMO+1 } \\
\text { HOMO-3 -> LUMO } \\
\text { HOMO-2 -> LUMO+1 }\end{array}$ & $\begin{array}{c}-0.12459 \\
0.11145 \\
0.67308 \\
\end{array}$ \\
\hline 9 & 3.2948 & 376.30 & 0.2080 & $\begin{array}{l}\text { HOMO-4 -> LUMO } \\
\text { HOMO-3 -> LUMO+1 } \\
\text { HOMO-2 -> LUMO } \\
\text { HOMO -> LUMO+2 }\end{array}$ & $\begin{array}{c}-0.10948 \\
0.28493 \\
-0.29037 \\
0.54085\end{array}$ \\
\hline 10 & 3.3511 & 369.98 & 0.0029 & $\begin{array}{l}\text { HOMO-4 -> LUMO+1 } \\
\text { HOMO-3 -> LUMO } \\
\text { HOMO-2 -> LUMO+1 }\end{array}$ & $\begin{array}{c}0.60228 \\
-0.31322 \\
0.16449 \\
\end{array}$ \\
\hline
\end{tabular}


Table S17. TD-DFT calculation of compound 6 at B3LYP-D3/6-311+G(2d,p) level in gas phase. (HOMO=158, LUMO=159).

\begin{tabular}{|c|c|c|c|c|}
\hline Excited state & Energy (eV) & Wavelength (nm) & Oscillator strength (f) & Description \\
\hline 1 & 2.7444 & 451.77 & 0.0158 & $\begin{array}{lr}\text { HOMO-1 -> LUMO } & 0.49400 \\
\text { HOMO -> LUMO+1 } & -0.47726 \\
\text { HOMO -> LUMO+2 } & -0.11041\end{array}$ \\
\hline 2 & 2.8036 & 442.23 & 0.2189 & $\begin{array}{ll}\text { HOMO-1 -> LUMO+1 } & 0.27991 \\
\text { HOMO -> LUMO } & 0.62103\end{array}$ \\
\hline 3 & 2.8887 & 429.21 & 0.0787 & $\begin{array}{lc}\text { HOMO-4 -> LUMO } & -0.10937 \\
\text { HOMO-3 -> LUMO+1 } & -0.12664 \\
\text { HOMO-2 -> LUMO } & 0.46879 \\
\text { HOMO-1 -> LUMO } & 0.25389 \\
\text { HOMO -> LUMO+1 } & 0.37806 \\
\text { HOMO -> LUMO+2 } & -0.18745 \\
\end{array}$ \\
\hline 4 & 2.9330 & 422.72 & 0.0958 & $\begin{array}{lr}\text { HOMO-4 -> LUMO+1 } & -0.15595 \\
\text { HOMO-3 -> LUMO } & -0.17161 \\
\text { HOMO-2 -> LUMO+1 } & 0.10411 \\
\text { HOMO-1 -> LUMO+1 } & 0.57242 \\
\text { HOMO-1 -> LUMO+2 } & -0.13184 \\
\text { HOMO -> LUMO } & -0.28987 \\
\end{array}$ \\
\hline 5 & 3.0023 & 412.97 & 0.1296 & $\begin{array}{lc}\text { HOMO-2 -> LUMO } & 0.45256 \\
\text { HOMO-1 -> LUMO } & -0.39154 \\
\text { HOMO -> LUMO+1 } & -0.33753 \\
\text { HOMO -> LUMO+2 } & -0.10715 \\
\end{array}$ \\
\hline 6 & 3.0250 & 409.87 & 0.0225 & $\begin{array}{lc}\text { HOMO-4 -> LUMO+1 } & -0.12660 \\
\text { HOMO-3 -> LUMO } & -0.22676 \\
\text { HOMO-2 -> LUMO+1 } & 0.60353 \\
\text { HOMO-1 -> LUMO+1 } & -0.23545 \\
\end{array}$ \\
\hline 7 & 3.0938 & 400.74 & 0.0161 & $\begin{array}{lc}\text { HOMO-4 -> LUMO } & 0.56402 \\
\text { HOMO-3 -> LUMO+1 } & 0.35780 \\
\text { HOMO -> LUMO+2 } & -0.17240 \\
\end{array}$ \\
\hline 8 & 3.1148 & 398.05 & 0.0242 & $\begin{array}{lc}\text { HOMO-4 -> LUMO+1 } & 0.29798 \\
\text { HOMO-3 -> LUMO } & 0.52277 \\
\text { HOMO-2 -> LUMO+1 } & 0.29456 \\
\text { HOMO-1 -> LUMO+1 } & 0.13684 \\
\text { HOMO-1 -> LUMO+2 } & -0.14602 \\
\end{array}$ \\
\hline 9 & 3.3518 & 369.90 & 0.2040 & $\begin{array}{l}\text { HOMO-4 -> LUMO } \\
\text { HOMO-2 -> LUMO } \\
\text { HOMO-1 -> LUMO } \\
\text { HOMO -> LUMO+2 }\end{array}$ \\
\hline 10 & 3.3814 & 366.67 & 0.0256 & $\begin{array}{lc}\text { HOMO-4 -> LUMO } & -0.35769 \\
\text { HOMO-3 -> LUMO+1 } & 0.58408 \\
\text { HOMO -> LUMO+2 } & 0.12767 \\
\end{array}$ \\
\hline
\end{tabular}


Table S18. The absolute energy (electronic and Gibbs free energy, i.e. E and G, unit: Hartree) for compound 2-6.

\begin{tabular}{lll}
\hline Entry & $\mathbf{E}_{\mathbf{B} 3}{ }^{a}$ & $\mathbf{G}_{\mathbf{B} 3}{ }^{a}$ \\
\hline 2-bb-gas & -1846.605012 & -1846.071328 \\
3-bb-gas & -1844.232219 & -1843.734026 \\
4-bb-gas & -1846.631399 & -1846.097039 \\
5-bb-gas & -1844.238996 & -1843.740540 \\
6-bb-gas & -1844.243508 & -1843.744904 \\
\hline
\end{tabular}

${ }^{a} \mathbf{E}_{\mathbf{B} 3}, \mathbf{G}_{\mathbf{B} 3}$ are obtained at B3LYP-D3/6-311+G(2d,p) in gas phase. 


\section{Computational details}

Gaussian $16^{[9]}$ was utilized for all the optimization and harmonic vibrational frequency (at 298.15 K) calculations. 6-31+G* basis set and SMD solvation model ${ }^{[10]}$ (solvent = dichloromethane/dichloroethane) was used for mechanistic reaction pathway explorations with the following parameters $(\operatorname{acc} 2 \mathrm{e}=11$, grid $=$ fine, constants $=2006)$. The frequency calculations were performed to ensure that one imaginary frequency for all transition states and no imaginary frequencies for all intermediates. IRC calculations were also carried out to connect the critical transition states to their corresponding reactant intermediate and product intermediate. Single point energy corrected with B3LYP ${ }^{[11]}-{ }_{-D}{ }^{[12]} / 6-311++G^{* * / / B 3 L Y P}-D 3 / 6-31+\mathrm{G}^{*}$ and $\mathrm{M} 06^{[13]} / 6-311++\mathrm{G}^{* * / / B 3 L Y P}-\mathrm{D} 3 / 6-31+\mathrm{G}^{*}$ were performed to confirm the B3LYP-D3/6-31+G* results. For TD-DFT and NICS computations, Gaussian 16 default integration settings $(\operatorname{acc} 2 \mathrm{e}=12$, grid $=$ ultrafine) were applied. TD-DFT and NICS calculations were carried out afer geomtry optimization at B3LYP-D3/6-311+G(2d,p) level in gas phas with gaussian 16 default settings. The 3D structures are generated with CYLview software ${ }^{[14]}$. 


\section{References}

[1] G.-L. Chai, J.-W. Han, H. N. C. Wong, Synthesis 2017, 49, 181-187.

[2] A. A. Gorodetsky, C.-Y. Chiu, T. Schiros, M. Palma, M. Cox, Z. Jia, W. Sattler, I. Kymissis, M. Steigerwald, C. Nuckolls, Angew. Chem. Int. Ed. 2010, 49, 7909-7912.

[3] Chaolumen, M. Murata, A. Wakamiya, Y. Murata, Angew. Chem. Int. Ed. 2017, 56, 5082-5086.

[4] (a) E. Clar, in Polycyclic Hydrocarbons: Volume 1 (Ed.: E. Clar), Springer, Berlin, Heidelberg, 1964, pp. 32-39; (b) Z. He, L. Shan, J. Mei, H. Wang, J. W. Y. Lam, H. H. Y. Sung, I. D. Williams, X. Gu, Q. Miao, B. Z. Tang, Chem. Sci. 2015, 6, 3538-3543.

[5] (a) P. Rempala, J. Kroulík, B. T. King, J. Am. Chem. Soc. 2004, 126, 15002-15003; (b) P. Rempala, J. Kroulík, B. T. King, J. Org. Chem. 2006, 71, 5067-5081; (c) B. T. King, J. Kroulík, C. R. Robertson, P. Rempala, C. L. Hilton, J. D. Korinek, L. M. Gortari, J. Org. Chem. 2007, 72, 2279-2288.

[6] (a) X. Yang, M. Hoffmann, F. Rominger, T. Kirschbaum, A. Dreuw, M. Mastalerz, Angew. Chem. Int. Ed. 2019, 58, 10650-10654; (b) M. Di Stefano, F. Negri, P. Carbone, K. Müllen, Chem. Phys. 2005, 314, 85-99; (c) L. Zhai, R. Shukla, S. H. Wadumethrige, R. Rathore, J. Org. Chem. 2010, 75, 4748-4760.

[7] (a) Y. Wu, C. Yan, D. Li, C. Yuan, J. Sun, S. Zhou, H.-L. Zhang, X. Shao, Chem. - Asian J. 2019, 14, 1860-1869; (b) Chaolumen, M. Murata, A. Wakamiya, Y. Murata, Angew. Chem. Int. Ed. 2017, 56, 5082-5086.

[8] Y. Wu, C. Yan, D. Li, C. Yuan, J. Sun, S. Zhou, H.-L. Zhang, X. Shao, Chem. Asian. J. 2019, 14, 1860-1869.

[9] M. J. Frisch, G. W. Trucks, H. B. Schlegel, G. E. Scuseria, M. A. Robb, J. R. Cheeseman, G. Scalmani, V. Barone, G. A. Petersson, H. Nakatsuji, X. Li, M. Caricato, A. V. Marenich, J. Bloino, B. G. Janesko, R. Gomperts, B. Mennucci, H. P. Hratchian, J. V. Ortiz, A. F. Izmaylov, J. L. Sonnenberg, Williams, F. Ding, F. Lipparini, F. Egidi, J. Goings, B. Peng, A. Petrone, T. Henderson, D. Ranasinghe, V. G. Zakrzewski, J. Gao, N. Rega, G. Zheng, W. Liang, M. Hada, M. Ehara, K. Toyota, R. Fukuda, J. Hasegawa, M. Ishida, T. Nakajima, Y. Honda, O. Kitao, H. Nakai, T. Vreven, K. Throssell, J. A. Montgomery Jr., J. E. Peralta, F. Ogliaro, M. J. Bearpark, J. J. Heyd, E. N. Brothers, K. N. Kudin, V. N. Staroverov, T. A. Keith, R. Kobayashi, J. Normand, K. Raghavachari, A. P. Rendell, J. C. Burant, S. S. Iyengar, J. Tomasi, M. Cossi, J. M. Millam, M. Klene, C. Adamo, R. Cammi, J. W. Ochterski, R. L. Martin, K. Morokuma, O. Farkas, J. B. Foresman, D. J. Fox, Wallingford, CT, 2016.

[10] A. V. Marenich, C. J. Cramer, D. G. Truhlar, J. Phys. Chem. B 2009, 113, 6378.

[11] (a) A. D. Becke, Phys. Rev. A 1988, 38, 3098-3100; (b) C. Lee, W. Yang, R. G. Parr, Phys. Rev. B 1988, 37, 785; (c) A. D. Becke, J. Chem. Phys. 1993, 98, 1372-1377; (d) P. J. Stephens, F. J. Devlin, C. F. Chabalowski, M. J. Frisch, J. Phys. Chem 1994, 98, 11623-11627.

[12] S. Grimme, J. Antony, S. Ehrlich, H. Krieg, J. Chem. Phys. 2010, 132, 154104-154119.

[13] Y. Zhao, D. G. Truhlar, Theor. Chem. Acc. 2008, 120, 215-241.

[14] C. Legault, 1.0b ed., Université de Sherbrooke Quebec, Canada, 2009. 


\section{Cartesian coordinates with charge and multiplicity of all the optimized structures for calculations}

\subsection{Calculations \\ B3LYP-D3/6-31+G(d) level}

\section{Compound 2}

01

C, $0.6769173133,5.209360408,-2.6288986101$ C, $1.9269280639,5.2160877801,-1.9563402386$ C, $2.237595575,4.2209332325,-1.0515084501$ C, $1.3118742748,3.1766901963,-0.7757288412$ C, $0.0478266599,3.1704286921,-1.4549073038$ C, $-0.241033993,4.2075549453,-2.3848202155$ C, $1.604092928,2.1340637207,0.1438838953$ C, $0.6818808305,1.1460525956,0.4188349251$ C, $-0.5845435441,1.1399903395,-0.2609985878$ C, $-0.8717384586,2.1216831059,-1.1862440815$ C, $0.9654664605,-0.0477278427,1.2653346632$ C, $0.6836046242,-1.256223799,0.4387316327$ C, $-0.5818326625,-1.262191726,-0.2428547203$ C,-1.437002483,-0.0606513362,-0.0260621352 C, $1.6079706614,-2.2463619587,0.1791634059$ C, $1.3188680028,-3.3034296129,-0.724562795$ C, $0.0554488078,-3.3093451095,-1.4049669131$ C, $-0.8665777936,-2.2582653375,-1.1533221678$ C, $2.2476496567,-4.3491151797,-0.9847647185$ C, $1.9392800291,-5.358719491,-1.8743875895$ C, $0.6891796976,-5.3650347751,-2.5467933378$ C, $-0.2309579354,-4.3617184418,-2.3181626534$ C, $-2.7412059601,-0.0605947913,0.3715430371$ C, $1.3545770352,-0.0363063477,2.5718573355$ C,-3.6151103188,-1.2458591489,0.6331594065 C,-4.9407396367,-0.7923333891,0.851541959 C, $-4.9421708803,0.6700946442,0.8446521465$ C, $-3.6182402048,1.1249330071,0.6193417551$ C, $1.6218712205,-1.2150436735,3.4519089611$ C, $2.1710431732,-0.7526565725,4.6745276887$ C, $2.1785046495,0.7097694467,4.661768489$ C, $1.6306104201,1.1556184927,3.4321716768$ C, $-5.9903126655,1.5579856965,1.09209711$ C, $-5.7158108044,2.9276552898,1.1516974872$ C, $-4.4018685057,3.3869785406,0.9851374449$ C, $-3.3534984519,2.496932953,0.7213475358$ C, $-3.34699621,-2.6155763969,0.7522884769$ C, $-4.393342323,-3.5054319554,1.0240895881$ C, $-5.7093062811,-3.0481458914,1.1816205242$ C, $-5.987363315,-1.680020983,1.1069665381$ C, $1.3763159487,-2.5861630532,3.3021731147$ C, $1.7279082231,-3.4690777023,4.3306083073$ C, $2.3210473151,-3.0032143443,5.5123158057$ C, $2.5361191945,-1.6335853488,5.6937350303$ C, $2.5537205176,1.6045748907,5.6653481853$ C, $2.3502897036,2.9725704729,5.4607710858$ C, $1.7570251374,3.4230068639,4.2729676253$ C, $1.3957786222,2.5258648123,3.2604253669$ $\mathrm{H}, 0.4452305832,5.9986252541,-3.3397399856$ H,2.6407492223,6.0111227038,-2.1572522733 H,3.1944447543,4.2226186093,-0.5341833621 H,-1.1998670716,4.1985122523,-2.8987525308 H,2.57324949,2.1218363159,0.635456299 H,-1.8162749425,2.1005990914,-1.7229315291 H,2.5766112085,-2.2255306376,0.6710716349
H,-1.8105572418,-2.2458463365,-1.691662029 H,3.2050270767,-4.3402772663,-0.4681258349 H,2.6553108847,-6.1544221314,-2.0643144952 H, $0.4586097832,-6.1662135149,-3.2446038296$ $\mathrm{H},-1.1899478967,-4.3630808778,-2.8315128328$ H,-6.999041289,1.1867994315,1.2556623921 H,-6.5178408617,3.6353724466, 1.3465845591 $\mathrm{H},-4.1868768833,4.4497324205,1.0658871047$ H,-2.3499079877,2.8864992407,0.6153298108 $\mathrm{H},-2.3424794102,-3.0044868449,0.6523428177$ H,-4.1752656132,-4.5665042547,1.117034369 H,-6.5101202858,-3.7557879468,1.3820389149 H,-6.9981714029,-1.3107989694,1.2636671296 H, $0.9048044431,-2.980212921,2.4117558885$ H, 1.5308584134,-4.5312427827,4.2074575715 $\mathrm{H}, 2.5954533736,-3.7055211559,6.2956222894$ $\mathrm{H}, 2.9615128191,-1.2569285667,6.6206882522$ $\mathrm{H}, 2.9796070709,1.2410975974,6.5976169515$ H,2.6335059729,3.6857163669,6.2311216236 H,1.5688619589,4.484469705,4.1317067144 H,0.9268348026,2.9097677629,2.3643318057

\section{Rearrangement product 4}

01

C,20.1512797377,30.2520220007,8.9495377354 C, 20.3247067092,29.7322571197,10.1875046299 C, $19.3384362272,29.712600633,11.3046019323$ C, $18.0361635781,30.2132942318,11.4153168915$ $\mathrm{H}, 17.5560007105,30.7195242186,10.5874408177$ C, $17.3423446859,30.0611706829,12.6227505685$ $\mathrm{H}, 16.3312486105,30.4519623077,12.7072404712$ C, 17.93333761648,29.4165499394,13.7183607775 H,17.3779195727,29.3063732912,14.6465075799 C, $19.2391941176,28.9230162539,13.6245152255$ H,19.7079187081,28.4346279867,14.4753641626 C, $19.9338315931,29.078035647,12.4238483489$ C, $21.3059180217,28.7013672846,12.0735361432$ C. $22.2925204329,28.0846948646,12.8447425783$ H,22.0853818582,27.7810636976,13.8680645266 C, $23.556117948,27.8738183372,12.2821790762$ $\mathrm{H}, 24.3374236131,27.3966481316,12.8686919685$ C, $23.8212264453,28.2844616546,10.968235379$ $\mathrm{H}, 24.809257846,28.1274509312,10.5424470714$ C,22.8307463998,28.8987718618,10.1910463463 $\mathrm{H}, 23.0652260866,29.209994951,9.1808219945$ C, $21.5587265917,29.1033897188,10.7377224872$ C, $18.9196133761,30.9154139489,8.4316182571$ C, 17.826008674,30.1798238822,8.0203158307 $\mathrm{H}, 17.8028176217,29.1071403075,8.1982442423$ C, 16.7317219483,30.7927731575,7.3564910314 C, $15.5873312286,30.0582243474,6.9366463289$ $\mathrm{H}, 15.5441465402,28.9912181303,7.1446058258$ C, $14.5521570134,30.6875324125,6.2761439978$ H,13.6820749558,30.117763175,5.9594145203 C, 14.6150512055,32.0801480682,6.0038815156 H,13.7926642179,32.5626905446,5.4816444811 C, $15.7117811275,32.818400886,6.3985849212$ H,15.7633736576,33.8856467806,6.1937959912 C, 16.7944460823,32.1990149915,7.084301545 C, $17.9297862307,32.932024369,7.5177555871$ $\mathrm{H}, 17.9626354891,34.0004885943,7.3192571737$ C, $18.9768630673,32.328851949,8.191869248$ C, $20.1387629353,33.1203059405,8.6975885427$ C, $19.889601351,34.2671474465,9.5577757802$ C, $18.5901094903,34.5567902307,10.0548997453$ $\mathrm{H}, 17.7705767853,33.8866798017,9.8254572377$ C, $18.3441764548,35.666006738,10.8435627871$ H, 17.3397597046,35.8558050041,11.2135420279 C, $19.3988614829,36.5385529393,11.169984658$ 
H,19.2141656063,37.4189123767,11.7804512854 C,20.6827272718,36.2580171146,10.7359884051 H,21.4809346954,36.928230369,11.0341472375 C,20.9701349082,35.1164158045,9.9452266176 C, $22.3316134039,34.7518000573,9.5774744699$ C, $23.4521502122,35.5289932378,9.9667121717$ H,23.3074847814,36.4692601636,10.4865998639 C, $24.7483400415,35.1141790121,9.7154644133$ $\mathrm{H}, 25.585318967,35.7315566233,10.0318837241$ C,24.9760138376,33.8814449154,9.0759882867 H,25.9904157179,33.526117064,8.9132061401 C,23.9023536071,33.1177677457,8.6553801282 $\mathrm{H}, 24.0945633978,32.1672289478,8.172894807$ C, $22.5605355095,33.5416525638,8.8547416449$ C, $21.4327546592,32.7652970885,8.3614119736$ C, $21.6946655384,31.5794939086,7.4907460843$ C,22.4664691732,31.6778917859,6.3463692651 $\mathrm{H}, 22.8643027313,32.6445825929,6.0476140792$ C, $22.7740367104,30.5455727559,5.5479554099$ C, 23.5785235797,30.6421355197,4.377685952 $\mathrm{H}, 23.9592861097,31.6176709415,4.0827646834$ C, $23.8740666625,29.5187986793,3.6330729022$ H,24.4905934271,29.6026961421,2.7415852838 C,23.3764788937,28.2473456941,4.0245692541 H,23.6172554791,27.3703632988,3.428814571 C,22.5911169014,28.1223899515,5.1521904793 $\mathrm{H}, 22.2059015966,27.1507156048,5.4539398824$ C,22.2695315646,29.2624524126,5.9409341581 C,21.4495234092,29.1799107186,7.0961714604 $\mathrm{H}, 21.0310818249,28.216856495,7.3795384688$ C,21.1654524426,30.2966467432,7.8566131481

\section{RCO}

01

C, $-0.0492615723,-0.9151076497,-2.3718324565$ C, $-0.2058998875,-0.3479146915,-3.6012702375$ C, $0.8669284661,0.1306971211,-4.526901385$ C, $2.2442000321,0.3271314355,-4.3625852553$ H, $2.7352178311,0.1222758172,-3.4208224783$ C,3.0066657122,0.8174192858,-5.4299128215 H,4.074327659,0.9694137914,-5.2918048107 C,2.4138315623, 1.1171372333,-6.6641606874 H,3.0238267723,1.4874132515,-7.4844186881 C, $1.0342502519,0.9639839809,-6.8324014606$ $\mathrm{H}, 0.5584842599,1.2268523557,-7.7740436286$ C, $0.2722592657,0.4909747613,-5.7626264639$ C, $-1.1793480338,0.35625811,-5.6407858758$ C,-2.1804173872,0.6655651801,-6.5630417073 H,- $1.9217503974,0.9995664533,-7.5648184782$ C,-3.5179697588,0.5632365869,-6.1688596543 $\mathrm{H},-4.3119030739,0.802179265,-6.8721250823$ C,-3.8345583424,0.1772792793,-4.8591217269 $\mathrm{H},-4.8746415029,0.1305409027,-4.5458928549$ C,-2.8301653794,-0.1466882181,-3.9387448209 H,-3.1136277566,-0.4235054068,-2.9324017011 C,-1.4861465931,-0.0881073498,-4.3296242777 C, $1.241458266,-1.1446032489,-1.6628846983$ C, $2.2630435672,-1.9278718743,-2.1570377634$ H,2.1958759477,-2.3319960384,-3.1635228914 C,3.4113933927,-2.2201258252,-1.3739603237 C, $4.4872733833,-3.004712241,-1.8739294172$ H,4.4294394403,-3.3866634933,-2.8908505128 C, $5.5866028323,-3.2746046378,-1.0836454305$ H,6.405215311,-3.8726972171,-1.4760120572 C,5.6567103924,-2.7760897168,0.2435184569 H,6.5277654787,-2.9975445683,0.8549942404 C, $4.6258812518,-2.015770863,0.7581828981$ $\mathrm{H}, 4.6735676957,-1.632815877,1.7752751537$ C,3.4828235889,-1.7166508036,-0.0330093615
C, $2.4044273208,-0.9405617558,0.4693560268$ $\mathrm{H}, 2.4571383295,-0.5855602227,1.4942469985$ C, $1.3138854486,-0.6386445077,-0.3185453796$ C, $0.0832584301,0.0531972588,0.1667992641$ C, $1.1500291479,2.2601542701,0.9527360505$ C, 2.5154542736,2.2148555134,0.6440046759 H,2.94635241,1.3840846172,0.1027579408 C,3.3465303417,3.2751439546,1.0260726074 $\mathrm{H}, 4.4046362357,3.2326556215,0.7803358053$ C,2.8344637009,4.3832441455,1.7138819061 H,3.4971389124,5.192150318,2.0111839718 C, $1.4675691398,4.4596149561,1.9975936874$ H, $1.0534989303,5.3288237867,2.5023124517$ C,0.6374296807,3.4097630751,1.6040000011 C,-0.8174805489,3.2915444124,1.6954849528 C,-1.7508023196,4.1982330354,2.1985890821 H,-1.4242469396,5.1290361343,2.6557112669 C,-3.1115937141,3.8968833466,2.0895581057 H,-3.8544000474,4.5907302948,2.4751310363 C,-3.5191144782,2.7107956982,1.4646120604 $\mathrm{H},-4.5785578604,2.492755707,1.356112955$ C, $-2.5816764168,1.7945630523,0.9730610536$ H,-2.9366578297,0.8958300383,0.4880862149 C,-1.2140298308,2.0654674482,1.1065199672 C, $0.0155443208,1.3169505946,0.6883377333$ C,-1.076114894,-0.8404712688,-0.1189361673 C, $-1.956932901,-1.2969307714,0.8382855083$ H,-1.9010004664,-0.9203987291,1.8553827957 C, $-2.9516595661,-2.2592164654,0.5203834535$ C, $-3.8801785606,-2.7288276169,1.4899260301$ H,-3.8286023614,-2.3307380711,2.5009493788 C,-4.829930544,-3.6721774193,1.1527554984 H,-5.5365466426,-4.0243590985, 1.9000362373 C,-4.8899032265,-4.1896603505,-0.1677083856 H,-5.6423891027,-4.9329684703,-0.4189150924 C,-3.9996573026,-3.755193176,-1.1292849237 H,-4.0417086316,-4.1501735049,-2.142035497 C,-3.0119257372,-2.7816798176,-0.8143162102 C,-2.0774472854,-2.3169212231,-1.7781306709 H,-2.1087412631,-2.7282963001,-2.7834880073 C,-1.1407602931,-1.3571085776,-1.4585099685 $\mathrm{H}, 0.3462161447,0.1474670611,2.5506452575$ S, $-0.1672105646,-0.2482432097,4.6352262181$ $\mathrm{O}, 0.3487641508,-1.2726399802,5.5272651563$ O,-1.5629708817,-0.1952805548,4.2194427445 C, $0.24274469,1.4124870486,5.4318366415$ F, 1.553455734,1.4844193312,5.6737072539 F, $-0.1195507695,2.4054605014,4.6191877707$ F,- $0.4304947976,1.5045949661,6.5828864887$ O,0.8108237593,-0.1915341864,3.3607493137

\section{TfOH}

01

H,-8.5475516333,0.7487365137,3.5949040784 S,-10.5641420999, 1.5124640666,3.8661140852 O,-10.1314408561,2.70439998997,3.1489128675 O,-11.3815983407,1.5593361396,5.0645920675 C,-11.4654704254,0.418168482,2.6197131928 F,-10.650581414,0.1576831413,1.5927396729 F,-11.8339130352,-0.7227359149,3.2020421602 F,-12.5438019027,1.0718325875,2.1828240946 O,-9.3029594627,0.5576601246,4.1989503909

\section{P1-TS1}

01

C, $-0.6455234293,0.973764935,-0.7814248445$ C, $-0.7737249344,2.3273348077,-0.8690224776$ C, $0.3248640973,3.3385138499,-0.9588691321$ 
C, $1.7203762189,3.2311940593,-0.8991081664$ H,2.2090915631,2.2742857693,-0.7806261699 C, 2.508406462,4.3863938065,-0.9744692757 $\mathrm{H}, 3.5903928235,4.29277576,-0.9229162595$ C, $1.9240339212,5.6525287893,-1.110860747$ H, 2.5525152506,6.5372779987,-1.1759854225 C, $0.5318531405,5.7796052166,-1.1447790429$ $\mathrm{H}, 0.0672356666,6.759347676,-1.2233988534$ C,-0.2533361151,4.6293676701,-1.0554910946 C,-1.7081320341,4.4999488651,-0.9729183398 C,-2.6884102759,5.4930614563,-0.9616099322 $\mathrm{H},-2.4149667026,6.5390013909,-1.0771841944$ C,-4.0244242489,5.1226468817,-0.7786088801 H,-4.8019373158,5.882438243,-0.7638318003 C,-4.3588384272,3.774207763,-0.5971780386 H,-5.3956653439,3.49145635,-0.4325359817 C,-3.376078084,2.7767899193,-0.6199916783 H,-3.6731859432,1.7493850192,-0.463137552 C, $-2.0358217258,3.128240241,-0.8260442424$ C, $0.6388916982,0.2182704303,-0.7084987817$ C, $1.5615116702,0.1303528666,-1.7277053028$ $\mathrm{H}, 1.427462795,0.7159378422,-2.633819508$ C, $2.7006107229,-0.7126597855,-1.6107983167$ C,3.6836285297,-0.7949150831,-2.6354979615 H,3.5577235522,-0.1913049675,-3.5317294882 C,4.7789422322,-1.6234116016,-2.4948229042 H,5.5258104501,-1.6773177498,-3.2829675086 C,4.936838046,-2.4096383782,-1.3244056235 H,5.8028877604,-3.0594114418,-1.2265514619 C,3.9970611348,-2.3535787348,-0.3141550452 H,4.1139608293,-2.9566983555,0.5836449905 C, $2.860604019,-1.5072552378,-0.4275027497$ C, $1.8751665244,-1.4299795433,0.5977590524$ H, $1.9963448337,-2.043982698,1.4858063467$ C, $0.8010129525,-0.5815015312,0.4751967966$ C, $-0.3589275414,-0.4494523472,1.4436161029$ C, $0.7759472518,1.3782469892,2.8676058454$ C,2.1516213166,1.2195830123,2.6743394712 $\mathrm{H}, 2.549329333,0.4076824569,2.0818255411$ C,3.0330310982,2.1324384598,3.2709271915 H,4.1027254319,2.0127624591,3.1232220587 C, $2.550469945,3.1889001545,4.0482214712$ H,3.247950759,3.8904127231,4.498440346 C, $1.1703518183,3.3509833555,4.259159952$ $\mathrm{H}, 0.796199783,4.1695345274,4.867780086$ C,0.2999381679,2.4422395596,3.6766455002 C, $-1.1661688513,2.3513452254,3.744162677$ C, $-2.0839138072,3.1601301048,4.3968538991$ H,-1.757400291,4.0257872915,4.9667233111 C,-3.4485187999,2.8363085788,4.3046693202 $\mathrm{H},-4.1829017394,3.4580333889,4.810109429$ C,-3.8678156071,1.7220960049,3.5726391415 H,-4.9246452436,1.4768016398,3.5163590329 C,-2.9398330601,0.9090767013,2.9073087737 H,-3.2885219508,0.0465220339,2.3581466253 C,-1.5803800351,1.2276146251,2.982577535 C, $-0.3877040076,0.622782161,2.3545746234$ C, $-1.6000385149,-0.7943829579,0.648719539$ C,-2.4854714573,-1.8080897512,0.9233101049 $\mathrm{H},-2.3818839716,-2.402138911,1.82601767$ C,-3.5727821442,-2.0814894208,0.0456836065 C,-4.5183972196,-3.1069888042,0.3189035295 $\mathrm{H},-4.4070794212,-3.6931365014,1.2285514558$ C, $-5.5593913648,-3.3552014258,-0.5537582141$ H,-6.27757476,-4.1412860531,-0.3345182781 C,-5.6989074104,-2.588672234,-1.7395338115 H,-6.5225796591,-2.7946227436,-2.4185280973 C,-4.7953128274,-1.5868155211,-2.032505119 H,-4.898332685,-0.996748938,-2.9406350911 C,-3.7147249343,-1.3047308648,-1.151810575
C, $-2.7705866243,-0.2766461176,-1.4247899428$ H,-2.8681645351,0.3009821154,-2.3405743084 C,-1.7487205323,-0.0028830457,-0.5428249431 H, $-0.1555611712,-1.4564399911,2.4109793162$ S,-0.6559763126,-2.6719629245,4.3754318606 O,-0.7154753534,-4.0867849207,4.7469059741 O,-1.8722963075,-1.8543071496,4.4144071917 C, $0.5164908917,-1.8802981678,5.6164239899$ F, 1.7021816831,-2.5075852278,5.6084335447 F, 0.7088646734,-0.5887597604,5.3096365822 F, $-0.008215116,-1.9602441441,6.8485525116$ O,0.1324979295,-2.4754958038,3.0580121613

\section{P1-INT1}

01

C,-0.0969975002,0.5604683224,-1.9784928185 C, $-0.2256168367,1.923118878,-1.921565149$ C, $0.8673361073,2.9373491515,-1.9360258275$ C, $2.2597623897,2.8301877653,-2.0398604824$ H, 2.7460170343,1.8734655832,-2.1601248546 C,3.0501496753,3.9849664915,-1.9729512594 H,4.1304622889,3.891154294,-2.0482559034 C,2.4706969738,5.247065822,-1.8029463912 H,3.099860494,6.1323462594,-1.7555124834 C, $1.0802108174,5.3711134019,-1.6871923472$ H, $0.6210558398,6.3456925874,-1.5424318997$ C, $0.2953885539,4.2225911347,-1.7491258005$ C, $-1.1526130522,4.0737892592,-1.5948385795$ C,-2.120923367,5.0401982454,-1.3334547901 $\mathrm{H},-1.8470077394,6.089423025,-1.2549068356$ C,-3.4498183579,4.6346885602,-1.1564456215 $\mathrm{H},-4.2195320817,5.3740815588,-0.9499535879$ C,-3.7855904559,3.2784337743,-1.2355946284 $\mathrm{H},-4.8153110771,2.9660969539,-1.0819892043$ C,-2.8120571157,2.3071883311,-1.5013759748 H, $-3.1097031885,1.2696767186,-1.5421691621$ C,-1.4805607738,2.6969427304,-1.6937743246 C, $1.1828588867,-0.2082444013,-1.998640177$ C, $2.0892453415,-0.2487768701,-3.0338095835$ H, $1.9545162684,0.3850258416,-3.9067386166$ C,3.211972994,-1.123689938,-2.9812092008 C,4.1781070876,-1.1643174096,-4.0236375662 H,4.051694852,-0.5065759298,-4.8808985787 C,5.2582681508,-2.021563466,-3.950082844 H,5.9917744041,-2.0430609396,-4.7521389021 C, $5.4172088391,-2.878572775,-2.8313811625$ H,6.2709807311,-3.5499639788,-2.7857903181 C, $4.4932486602,-2.8647217284,-1.8047098076$ H,4.6102095868,-3.5223466635,-0.9461647475 C,3.3722743391,-1.9926313471,-1.8501217573 C, $2.4003710186,-1.9579029406,-0.8080214028$ $\mathrm{H}, 2.5105244534,-2.6185639846,0.0488554858$ C, $1.3425046909,-1.087331079,-0.8763323314$ C, $0.2070330068,-1.0195864719,0.1370309329$ C, $1.2448223689,1.1864888588,1.1608418424$ C, 2.6224449521,1.0666114585,0.9285772196 H,3.0390026116,0.1958153556,0.4417062919 C,3.4735228692,2.0993362203,1.3496265931 H,4.5415831118,2.0136669823,1.1742330547 C,2.9593539253,3.2334885421,1.9770948091 H,3.6314397128,4.0296695237,2.2854597717 C, $1.5731031044,3.3655190394,2.2220349558$ H, 1.1837029307,4.2494034752,2.7188579724 C, $0.7378384039,2.3441133152,1.8235629705$ C,-0.7175527991,2.1689511575,1.9760968294 C,-1.6703239796,2.9740484376,2.5613061644 H,-1.4089700211,3.9375788176,2.9892884726 C,-3.0060348751,2.5108555919,2.6022463549 H,-3.7690847295,3.1339465365,3.0607411548 
C, $-3.3557602773,1.2692603275,2.0710535006$ $\mathrm{H},-4.3847184874,0.9267573888,2.1238281892$ C, $-2.3900795226,0.4555330816,1.4627649268$ $\mathrm{H},-2.6720188615,-0.5081161872,1.0650388454$ C, $-1.0631285845,0.907593787,1.4044689778$ C, $0.1308841151,0.3070730914,0.8489979008$ C,-1.0368845549,-1.3092268942,-0.697595256 C,-1.9019735379,-2.3531295369,-0.4894692697 H,-1.7786886206,-2.9974905032,0.3762866068 C,-2.9930560545,-2.5693529636,-1.3800417557 C,-3.9260325153,-3.622733986,-1.1807575267 $\mathrm{H},-3.7974468036,-4.2797582878,-0.3234812178$ C, $-4.9768947494,-3.8084796259,-2.0574689385$ $\mathrm{H},-5.6850201698,-4.6170262109,-1.8944609345$ C, $-5.1395499732,-2.9484045399,-3.1736179179$ $\mathrm{H},-5.9702250193,-3.1060584455,-3.8570342323$ C,-4.2485147171,-1.9168724486,-3.3947227817 $\mathrm{H},-4.3698875695,-1.2553170362,-4.2497640814$ C, $-3.1587699154,-1.6972932807,-2.5081223863$ C, $-2.2293808632,-0.637623555,-2.7093171024$ H,-2.3424608629,0.008764549,-3.5761209044 C,-1.2021096346,-0.4291732552,-1.8167963018 $\mathrm{H}, 0.3403273872,-1.7845235587,0.9161341052$ S, $-0.2654977196,-2.9692219504,3.4219071329$ O,-0.6336481215,-4.0468004741,4.3671071384 $\mathrm{O},-1.3965820117,-2.2398377618,2.7997138727$ C, $0.5080858603,-1.6630406695,4.5373320504$ F, $1.5573438795,-2.1632323226,5.2234704319$ F, 0.9552971654,-0.6132232774,3.8124746033 F,- $0.3834065815,-1.1912490841,5.4342449165$ $\mathrm{O}, 0.8437247957,-3.288794449,2.4849094545$

\section{P1-TS2}

01

C,0.6510343134,-8.6281192421,-1.7146801208 C, $-0.5709859034,-9.0654626528,-2.1428705748$ C,-0.9119796136,-10.348767285,-2.831762908 C, $-0.127564599,-11.36445914,-3.3943735968$ $\mathrm{H}, 0.9534474089,-11.3303178529,-3.3634294806$ C, $-0.7444773757,-12.4472596803,-4.0323176868$ $\mathrm{H},-0.1257502773,-13.2283192877,-4.4668546126$ C, $-2.1403251767,-12.533824373,-4.1186551046$ H,-2.6028021556,-13.3888861043,-4.6052053381 C,-2.9371281239,-11.5059843278,-3.604856593 H,-4.0191508952,-11.5411300918,-3.704855487 C,-2.3192003065,-10.4169538273,-2.9894633077 C,-2.906686287,-9.1654385935,-2.511094444 C, $-4.235754866,-8.743558675,-2.5377577418$ $\mathrm{H},-5.0196920177,-9.4141441232,-2.8802620584$ C, $-4.5380453328,-7.4361917159,-2.1429463912$ $\mathrm{H},-5.5673418043,-7.0869241188,-2.1610785523$ C,-3.510698045,-6.5681132924,-1.7521311147 H,-3.7440383304,-5.5420082864,-1.4794060864 C,-2.1782653975,-6.9973962688,-1.7111980686 H,-1.4155712049,-6.2877525056,-1.4228235338 C,-1.8631338292,-8.3166097656,-2.0636116415 C, $1.9295509011,-9.3829028858,-1.7472219735$ C, $2.0132103832,-10.6774248879,-1.2809234072$ H, $1.1100520855,-11.1890780576,-0.9635463304$ C,3.2502787122,-11.3691394889,-1.166600985 C, $3.3178948447,-12.7183583088,-0.7361263258$ $\mathrm{H}, 2.3956239922,-13.2460312181,-0.505681452$ C,4.5410262872,-13.3530263762,-0.6197188346 H,4.5830786108,-14.3904597508,-0.2983594148 C,5.746377463,-12.6645084366,-0.911819714 H,6.6976405021,-13.1793162131,-0.8081172142 C,5.7132754019,-11.3471343093,-1.3212797518 H,6.6312577187,-10.8074264932,-1.5398992706 C,4.4686339351,-10.6731645933,-1.4620109727
C, $4.3912386132,-9.3167617352,-1.8413676639$ H,5.309856307,-8.7600503096,-1.9974437024 C,3.1630591521,-8.6782330587,-2.0336231331 C,3.2519526386,-7.3013923468,-2.4458730207 C, $1.3053556391,-6.8467719906,-4.2190894136$ C, $0.8532867232,-8.0913847493,-4.6415274253$ H,1.2130684342,-9.0062035782,-4.1939950585 C,-0.0640821657,-8.1674975578,-5.7002751454 H,-0.4284892158,-9.1416049329,-6.0145569606 C,-0.5034696542,-7.0090989997,-6.3484685733 H, $-1.2278470861,-7.081553523,-7.1556714283$ C, $0.0377004501,-5.7635270528,-6.0065504507$ H,-0.2223437618,-4.8720241741,-6.5711721509 C, $0.9659624613,-5.6954268778,-4.9676038969$ C, $1.8847935975,-4.607690252,-4.609612706$ C,2.0529092127,-3.3444322088,-5.1780242725 H,1.3573609309,-2.9790200376,-5.9292096069 C,3.1570889699,-2.5739366341,-4.7911635581 H,3.3085104575,-1.5910263341,-5.2301380984 C,4.0847327386,-3.0736998018,-3.8695827538 H,4.9591903137,-2.4858108502,-3.6041314643 C,3.9038776251,-4.3356289624,-3.2818565333 H,4.6390968276,-4.697807942,-2.5701929729 C, $2.7872680219,-5.0837279073,-3.6394157594$ C, 2.2966416696,-6.4390981497,-3.1312148569 C, $1.740614137,-6.3152622931,-1.6180149562$ C, $2.0922501425,-5.2055146827,-0.8449681848$ H, 2.7781123143,-4.4630482888,-1.2292125599 C, $1.5500313814,-4.9914199688,0.4384257084$ C, $1.892819898,-3.8434093867,1.208351415$ H, 2.5878468095,-3.1189322199,0.7913636237 C, $1.3488942185,-3.6638064962,2.4625722935$ H,1.6092901261,-2.7881279248,3.0506113303 C, $0.4475536156,-4.6222712197,2.9944214755$ H, $0.0265108235,-4.4664921508,3.9842317534$ C, $0.1015086958,-5.7487495387,2.270593965$ H, $-0.58603327,-6.4831817148,2.6823290634$ C, $0.6415134243,-5.9580888324,0.9780787256$ C, $0.3383579851,-7.1104452553,0.2033272917$ $\mathrm{H},-0.3179652087,-7.8654511254,0.6273531367$ C, $0.8555981238,-7.3080826908,-1.0554870413$ H,4.1978869977,-6.8261524003,-2.1890566912 S,7.0755966052,-5.8092588507,-1.1764179684 O, $8.1891683491,-6.0170175207,-0.2246057063$ O,6.918286687,-4.4317455718,-1.6995602175 C, 7.6217464176,-6.777460452,-2.6982372116 F,7.8429356286,-8.0767624948,-2.3973218256 F,6.671950167,-6.7390595055,-3.6604608071 F,8.7585727574,-6.2763576784,-3.2203199099 O,5.8001355649,-6.4850547304,-0.8188216766

\section{P1-INT2}

01 C,-3.6531456341,-6.780557635,8.1504467782 C, $-4.030202345,-5.9438827843,9.1536691256$ C,-5.0857828446,-4.8853389028,9.1090714924 C, $-5.8492222921,-4.3522515055,8.0630849007$ H,-5.7367431514,-4.6938667756,7.0429231079 C,-6.7714216371,-3.332333699,8.3314826595 H,-7.3585500896,-2.9213458708,7.5141536739 C,-6.9392049208,-2.8341423747,9.629927671 H,-7.6640772141,-2.0464717473,9.8194179304 C,-6.1582550508,-3.3317171421,10.6785574107 H,-6.2596672257,-2.9301194325,11.6838145678 C, $-5.2316910886,-4.3392570903,10.409058122$ C,-4.2397398501,-4.9562755958,11.2923880186 C,-3.958565672,-4.696058466,12.6339192357 $\mathrm{H},-4.5545152075,-3.9792379923,13.1931677$ C, $-2.8845483805,-5.3569757692,13.2392849724$ 
$\mathrm{H},-2.64813134,-5.1648812113,14.282887329$ C,-2.1007157247,-6.2506691226,12.4975851208 H,-1.2516765157,-6.7423844486, 12.9656629563 C,-2.3896979773,-6.520419174,11.1538385906 $\mathrm{H},-1.7530619218,-7.2041134272,10.6088504723$ C, $-3.480415602,-5.8893512998,10.5433800398$ C,-4.2656419755,-6.7465795448,6.7977854124 C, $-5.5330302205,-7.1927718034,6.5439512336$ H,-6.1168135708,-7.6468947822,7.3401758668 C,-6.1505644882,-6.9995184129,5.2666805758 C, $-7.4584270455,-7.4525625445,4.9922707184$ $\mathrm{H},-8.0009403119,-8.0031100843,5.7563315091$ C, $-8.0449323605,-7.1924224888,3.7627239459$ H,-9.0522517906,-7.5492867645,3.5645569645 C, $-7.3582562893,-6.4645007712,2.7588315235$ H,-7.8439554501,-6.2722828463,1.8065595907 C,-6.0803548233,-6.0050757371,2.9940991794 H, $-5.5339056927,-5.4428843495,2.2415026046$ C,-5.4492685495,-6.2630529247,4.2468912106 C, $-4.1495297898,-5.8096428561,4.5108860197$ H,-3.6130791705,-5.25819162,3.743877823 C,-3.5076555366,-6.1234962775,5.7332773839 C,-2.1337647107,-5.8784854922,5.7823889559 C, $-0.3152748948,-5.315891976,7.2499533125$ C, $-0.7204085137,-4.5387519192,8.3317515445$ H,-1.6520552923,-4.742079229,8.8466203345 C, $0.1150177681,-3.4981058177,8.7523017444$ H,-0.1750287436,-2.8912229486,9.60588627 C, $1.319064106,-3.2306011833,8.0814627737$ H, 1.9496957526,-2.4119527222,8.4185395978 C, $1.7160788615,-4.0010117644,6.9845516445$ H,2.6448233968,-3.7874347398,6.4622135011 C, $0.89187713,-5.0503256953,6.5672295055$ C, $1.0508941702,-6.0159018296,5.481052318$ C, $2.0675248665,-6.1744904348,4.5338009052$ H,2.9202857109,-5.5010469572,4.519470635 C, 1.9610494047,-7.2119373712,3.6033338156 H,2.7420856468,-7.3465266035,2.8594828104 C, $0.8559651845,-8.0788657166,3.6097745542$ $\mathrm{H}, 0.7916902062,-8.8759383153,2.8739919639$ C, $-0.1684448901,-7.9180620288,4.5480708758$ $\mathrm{H},-1.0276377473,-8.5833872134,4.5565691443$ C, $-0.0505871112,-6.8946351384,5.4851976873$ C, $-1.0501538853,-6.5100146949,6.5991923365$ C,-1.3559639772,-7.677451361,7.5465975406 C, $-0.3723946032,-8.6302791872,7.7495002283$ $\mathrm{H}, 0.582227279,-8.5393859539,7.2406349878$ C, $-0.5607446887,-9.7499309806,8.5992123724$ C, $0.4558202457,-10.7261298729,8.7997379824$ $\mathrm{H}, 1.4120338339,-10.6002243021,8.2966832633$ C, $0.2296013789,-11.8128692961,9.6184060174$ H,1.0108551348,-12.553872984,9.7676899897 C,-1.022103825,-11.9737971308,10.2716443499 $\mathrm{H},-1.1840564319,-12.8357695128,10.9139027505$ C, $-2.0259853181,-11.0444563205,10.0961658528$ H, $-2.9846850403,-11.1612563459,10.5965624318$ C,-1.8203556548,-9.9117776209,9.259080328 C, $-2.8082068832,-8.9162376734,9.0676714608$ H,-3.7559563738,-9.0070217388,9.5924746382 C,-2.5838832234,-7.8072218091,8.2708658066 $\mathrm{H},-1.7507105722,-5.1819506242,5.0363609312$ S, $-1.8881367009,-3.4413689087,2.2464811359$ O,-1.4817766198,-2.1849069985, 1.5801675554 $\mathrm{O},-3.257302595,-3.9245733836,1.9323280772$ C, $-0.8004612361,-4.7416546398,1.4295535212$ F,-1.0912873607,-5.9718741183,1.9040266683 F,-0.984295018,-4.7630359823,0.0920903948 F, $0.5067041919,-4.5062982161,1.662662937$ O,-1.5115886573,-3.5557125721,3.6789869899

\section{P1-TS3}

01

C,-1.9922511035,0.4864959711,-0.5509700042

C, $-2.9461800084,0.9466479296,0.3079444781$

C, $-3.0754154947,2.3245909283,0.8779001219$

C, $-2.2265646774,3.4390173615,0.8672493904$ $\mathrm{H},-1.2645401655,3.4145124782,0.3742700796$ C,-2.617513775,4.6127042834,1.5245509181 H,-1.9514822078,5.4717788079,1.5136425423 C, $-3.8459262432,4.6905768316,2.1932531222$ H,-4.1371615022,5.61344888423,2.6886172874 C,-4.6867761264,3.5738454218,2.2465160406 $\mathrm{H},-5.6250521305,3.6130793265,2.7945004631$ C,-4.2888585591,2.399161174,1.6075972366 C, $-4.9095849919,1.0733587978,1.6237974301$ C,-6.0599148393,0.6402038604,2.2842093818 H,-6.6858288856,1.3472960272,2.8225674821 C,-6.3774041187,-0.7217354541,2.2651802775 H,-7.2677242157,-1.0792379116,2.7764798237 C, $-5.5322974115,-1.6295811711,1.6134069345$ H,-5.7616415025,-2.692027931,1.6327944278 C,-4.3862554387,-1.1920837404,0.9374123238 $\mathrm{H},-3.7492222667,-1.9276383454,0.4651775844$ C, $-4.0809131831,0.1749343196,0.9058403843$ C, $-0.8746928072,1.320873286,-1.0680620829$ C,-1.1436938939,2.4012510368,-1.8844609596 H,-2.1756139315,2.629734494,-2.1378484033 C,-0.1176978225,3.2593445432,-2.358871224 C,- $-0.3908936894,4.388745428,-3.1778401735$ H,-1.4194115742,4.5935418916,-3.4661069786 C, $0.6349192757,5.2127060997,-3.5955034933$ $\mathrm{H}, 0.4185033773,6.0748210953,-4.2213291946$ C, $1.9764709725,4.9457693721,-3.2139676184$ H,2.7715153203,5.604918914,-3.5527774149 C, $2.2721160502,3.857081745,-2.4200395239$ H,3.2970306261,3.644855352,-2.1250043301 C, $1.2354195824,2.9910799967,-1.9732157645$ C, $1.5007090174,1.8530289585,-1.1729656185$ H,2.5319529486,1.6203129587,-0.9229790332 C, $0.4875574154,1.0065825064,-0.7442132716$ C, $0.9484264379,-0.2478289776,-0.1069876265$ C, $0.475365608,-0.7603019924,1.4405629569$ C, $-0.3201522231,0.15610409,2.1655424268$ $\mathrm{H},-0.9037792006,0.9064431132,1.6583624653$ C, $-0.2624187173,0.1631124133,3.5507216778$ $\mathrm{H},-0.8524514356,0.8881640542,4.1033222385$ C, $0.5738431947,-0.7373194388,4.2334094793$ $\mathrm{H}, 0.6087276604,-0.7189260611,5.3193323763$ C, $1.3548336059,-1.6579657577,3.5358541252$ $\mathrm{H}, 1.9810192824,-2.3672238697,4.0687594123$ C, $1.3032100616,-1.6904054127,2.1432529888$ C, $1.9304179936,-2.6714103413,1.2682891276$ C,2.9992946234,-3.5307607892,1.5434050433 H,3.4398152316,-3.5613442025,2.5356934637 C, 3.5078738912,-4.3211798342,0.5110973242 H,4.3410673143,-4.9909208497,0.7045730145 C, $2.964689131,-4.2389265291,-0.7782543295$ H,3.3756702112,-4.8498996498,-1.5773125285 C, $1.8987468067,-3.3730365607,-1.0555715258$ H, $1.4921849972,-3.3026278154,-2.0598038155$ C, $1.3674943595,-2.6157767368,-0.0147190529$ C, $0.3081314686,-1.54283972,-0.151715106$ C,-1.01380727,-1.8794047162,-0.7326800937 C,-1.2573743958,-3.2100799606,-1.0579897006 $\mathrm{H},-0.5610414218,-3.9812826578,-0.7510017449$ C, $-2.3741362463,-3.6114434948,-1.8298552057$ C,-2.5967066052,-4.9732095193,-2.1783337565 $\mathrm{H},-1.9012517037,-5.7288761736,-1.8204643843$ C,-3.6765942837,-5.3233400678,-2.9618877282 
$\mathrm{H},-3.842996049,-6.3642080252,-3.2266624209$ C, $-4.5761450466,-4.3291269403,-3.4298387038$ $\mathrm{H},-5.4221477526,-4.6215885618,-4.0465541879$ C,-4.3853653224,-3.0006621015,-3.1056698373 $\mathrm{H},-5.0768963954,-2.2384191282,-3.4568746469$ C, $-3.2825845391,-2.6113190764,-2.2994375927$ C,-3.0755067624,-1.2715680401,-1.8840021368 $\mathrm{H},-3.813897554,-0.5215327386,-2.1545127019$ C,-2.0160698694,-0.9032551009,-1.0789132655 H, 2.034021296,-0.3005616201,-0.0763313315 S, $4.7566628623,0.0078055018,0.859669667$ O,5.8899042389,-0.8572863278,1.2575161148 O,3.7197046321,0.2308520494,1.8946240456 C, $5.5552684546,1.7061021595,0.6917329478$ F,6.5366022242,1.6982981462,-0.2357491099 F,4.6470415214,2.6356006639,0.3172961317 F,6.0970728274,2.1111509059,1.8598679609 O,4.2196860085,-0.227959248,-0.5061933394

\section{P1-INT3}

01

C, $-0.0423115636,0.4738308716,-1.0943165145$ C,0.0052254013,-0.6898931828,-1.786116979 C,-1.1479686994,-1.526489994,-2.2236959722 C,-2.5303198477,-1.352229156,-2.1013209762 H,-2.9470071032,-0.4669099814,-1.6378527508 C,-3.3911046241,-2.3463137424,-2.5844202811 $\mathrm{H},-4.4655053601,-2.2124641982,-2.4864260354$ C, $-2.8863498751,-3.5058883994,-3.1885402815$ $\mathrm{H},-3.5709145731,-4.2651734489,-3.5584621558$ C,-1.5049306665,-3.6926136567,-3.3140931751 $\mathrm{H},-1.1079901276,-4.5936343171,-3.7751225029$ C, $-0.6465684134,-2.705114844,-2.8302313775$ C, $0.8185108002,-2.6578687863,-2.8065157471$ C, $1.7542434669,-3.585571826,-3.2640437669$ $\mathrm{H}, 1.4312018659,-4.5082490672,-3.7397462882$ C,3.1163399955,-3.3103125361,-3.0953270603 H,3.859627916,-4.022687478,-3.4446817749 C,3.5259843275,-2.1217147182,-2.4765316153 H,4.585956185,-1.9170792656,-2.3481500819 C, $2.5871877599,-1.1867606171,-2.0199208972$ H,2.9325795933,-0.2751242127,-1.5490191902 C, $1.2230918055,-1.4502014496,-2.1843991561$ C,-1.2606017909,1.1766150656,-0.6060528558 C,-2.0096481988,1.9988169295,-1.4226417179 $\mathrm{H},-1.8057078184,2.0261643338,-2.4904490764$ C, $-3.0300183054,2.8321162287,-0.8912825059$ C,-3.8286452064,3.6737714601,-1.71466064 H,-3.6629641415,3.6669902906,-2.7898397342 C, $-4.7957172677,4.4858851998,-1.1578002887$ H,-5.4019290603,5.1252955023,-1.7946972921 C,-5.0056110549,4.4950050344,0.2464070957 $\mathrm{H},-5.7700941707,5.1410423596,0.6706346198$ C, $-4.2449358279,3.6908396942,1.0705056431$ $\mathrm{H},-4.4010937945,3.6935381878,2.1468741237$ C,-3.2425375911,2.8410007509,0.5262598255 C, $-2.4563733053,1.9875490674,1.346569756$ $\mathrm{H},-2.6393165561,1.9779105669,2.418744268$ C,-1.5016536613,1.1515471102,0.8059775167 C,-0.7950771691,0.1405816938,1.6970009855 C,-1.5975857432,-1.1027300455,2.0161359293 C, $-2.988161078,-1.1418566515,1.840908158$ H,-3.5047270162,-0.2924863494,1.4119732439 C,-3.7229696379,-2.2688853127,2.1943827203 $\mathrm{H},-4.7973977842,-2.2816060028,2.0318482711$ C, $-3.0782193306,-3.3853045816,2.7446263001$ $\mathrm{H},-3.6466300728,-4.2708961629,3.0149810182$ C,-1.7053274219,-3.3607095449,2.9327151091 H,-1.2205149397,-4.2414539242,3.3379680316
C, $-0.9402602034,-2.2239794403,2.5801752807$ C, $0.5100721065,-2.2091366333,2.7340240778$ C, $1.1938083979,-3.2107558983,3.4487497398$ H, $0.6403283472,-3.9598893173,4.0015357883$ C, $2.5805341749,-3.2686605761,3.4631371335$ H,3.0726750524,-4.0583135752,4.024608951 C,3.3543875732,-2.3405519892,2.7371627097 H,4.4362572361,-2.4281372149,2.7135908822 C, $2.7238429776,-1.3207670113,2.0617003198$ H,3.3152596251,-0.6119416931,1.4975138327 C, $1.2987154405,-1.1821729091,2.0963398468$ C, $0.6636854364,-0.064062774,1.5127775574$ C, $1.4409665058,0.9861182892,0.8346309203$ C, $2.4390555318,1.7147180763,1.4605349854$ H, 2.652205722,1.5527798589,2.513575907 C,3.1702031955,2.6975334017,0.7472906516 C,4.1880671637,3.4714805083,1.371822961 H,4.3923846645,3.3135556084,2.4282017968 C, $4.9015456288,4.4034389453,0.647631516$ H,5.6774030404,4.9916712049,1.1305126238 C,4.6261265948,4.6025395043,-0.7304954342 H,5.1955464456,5.3413673471,-1.288576122 C,3.6416667733,3.8698076987,-1.3631108862 H,3.4279486521,4.0242852398,-2.4181461156 C, $2.8875226711,2.9032638214,-0.6450007568$ C, $1.855817261,2.1423107611,-1.262196977$ H, 1.6332091576,2.3035815585,-2.314251768 C, $1.1393107064,1.2110309673,-0.5477820555$ H, $-0.6962262808,0.644891272,2.7074131754$ S, $-1.3379901304,1.5868432786,5.4037686597$ O,-0.9630333108,2.4859870828,6.5160798022 O,-2.6629136188,1.8270657562,4.786948918 C, $-1.5570509193,-0.0803703085,6.2513365201$ F,-1.9297119731,-1.0292393493,5.3690218566 F,-0.4060511379,-0.4852975065,6.8300070714 F,-2.5016287726,-0.0146699506,7.2126537459 O, $-0.2432779433,1.3107334324,4.4289280903$

\section{P1-TS4}

01

C, $15.6024911916,9.5245237168,18.0346480362$ C, $16.6526962813,9.1218518538,18.78810777$ C, $17.8165827996,8.3011137838,18.3481227328$ C, 18.1460035932,7.7294747361,17.1143983314 H, 17.5125611217,7.861505051,16.2463839509 C, $19.316649529,6.9682930431,17.0023947165$ $\mathrm{H}, 19.571680969,6.523635011,16.0436744895$ C,20.1586738864,6.773847826,18.1060592989 H,21.0644626841,6.1822545309,17.9984426223 C, $19.8340924112,7.3314524251,19.3477929571$ H,20.4771183289,7.1739821483,20.2102264576 C, $18.6656827216,8.0857491315,19.4621557482$ C, $18.0747833811,8.7375508495,20.634653405$ C, $18.5231296447,8.7865536586,21.955080863$ H,19.4578369799,8.3094384488,22.2391639251 C, $17.7448587579,9.4533288945,22.908060147$ H, 18.0779641989,9.501196702,23.9418615029 C, $16.5349033422,10.0548761055,22.535985059$ $\mathrm{H}, 15.9330396668,10.5650667481,23.283985085$ C, $16.0858286356,10.0109154435,21.2095126321$ $\mathrm{H}, 15.1475343684,10.4853642377,20.9514732007$ C, 16.8590640617,9.354204427,20.2458222806 C, $15.3846508815,9.2457262863,16.5873625175$ C, $16.046796459,9.9638272637,15.6119607228$ $\mathrm{H}, 16.8428458915,10.6471560555,15.8980060651$ C, 15.700230079,9.8426468913,14.2408658239 C, $16.3799929193,10.5602472921,13.2175559888$ H,17.2027894211,11.2160931467,13.4937195476 C, $16.0004987665,10.4260997433,11.8975533646$ 
$\mathrm{H}, 16.5258720857,10.9778207367,11.1220013107$ C, $14.9239590235,9.5710848625,11.5415246398$ $\mathrm{H}, 14.6360321662,9.4769984739,10.4975316704$ C, $14.2456013322,8.863237323,12.5124152071$ H,13.4204833444,8.2062913815,12.2462986862 C, $14.6150297858,8.9792618161,13.8813821816$ C,13.9499142625,8.2503484467,14.9018488741 $\mathrm{H}, 13.1528960524,7.5718236024,14.6124792799$ C, $14.3201608619,8.3530664028,16.2284751829$ C, 13.6859553582,7.4676499511,17.2789020987 C, $13.9705247214,6.0100592966,17.2060533574$ C,14.6215313584,5.4381905046,16.0932603584 H,14.985539538,6.074063696,15.2970527209 C,14.8161714621,4.0696543911,15.9991764079 $\mathrm{H}, 15.3296546831,3.6567001827,15.1350056393$ C, $14.3506232778,3.2228619214,17.0185791843$ $\mathrm{H}, 14.489342233,2.1475765094,16.9451982287$ C, $13.7276875914,3.7635769629,18.1297728337$ H,13.3982748083,3.0923011129,18.9140403599 C, $13.5337227339,5.1606421673,18.2593909883$ C, $12.9529977806,5.7435428179,19.4631650681$ C, $12.4694826254,4.9437129787,20.5233093491$ $\mathrm{H}, 12.4548617549,3.8649297339,20.4281745743$ C,12.0150420926,5.5047110366,21.705479283 $\mathrm{H}, 11.6552996252,4.8571600608,22.500796602$ C,12.0393642661,6.9000702301,21.8909943029 $\mathrm{H}, 11.7203233154,7.3346872842,22.8338486645$ C, $12.4730403863,7.7132544072,20.8645627066$ $\mathrm{H}, 12.501314189,8.7845757117,21.0156161033$ C, $12.8978055292,7.1675902532,19.6189356699$ C, $13.2954322946,8.0227661747,18.5387018302$ C, $13.197621421,9.4954368018,18.6797372818$ C, $12.0060922614,10.133842269,18.9736338027$ H,11.0907986278,9.5571761006,19.0766929049 C,11.9523528907,11.5419051315,19.134667706 C, $10.7350928037,12.2169425232,19.4303677416$ H,9.8208904298,11.6346142706,19.5195301025 C,10.7169911682,13.5852838079,19.602270146 H,9.7834790007,14.0940452014,19.8282403314 C,11.9149486819,14.3381492949,19.4828147887 H,11.8855501903,15.4161757721,19.6196755063 C, $13.1102163888,13.7122548383,19.192892716$ H,14.0279702495,14.2881582988,19.0977928272 C, $13.1623056486,12.3028644876,19.0100134447$ C, 14.3714437417,11.6264866809,18.6941068166 H, 15.2865608082,12.2016596062,18.5756336409 C, 14.3991684971,10.2593523312,18.5273972235 $\mathrm{H}, 12.401786078,7.4822855426,16.9625189767$ S, $10.4350519252,6.6398790325,15.6185080307$ $\mathrm{O}, 11.4147571158,5.8205651858,14.8897810948$ $0,9.3501607371,7.277038429,14.8604104669$ C,9.5591767903,5.4341193716,16.7665547306 F, 10.4422314193,4.8042096822,17.5584650448 F, 8.6728970668,6.0788526457,17.545291775 F,8.8999863533,4.5117140686,16.0437189106 $\mathrm{O}, 11.0801060728,7.5979959139,16.6167958229$

\section{P1-INT4}

01

C, $1.7811413356,1.3546845361,0.3497259438$ C, $2.8796969357,1.044610655,1.0775529882$ C, $4.108059029,0.3448908157,0.6047620839$ C,4.456251456,-0.2072913217,-0.6331757173 H,3.7796808994,-0.1683179875,-1.4776320853 C,5.7039446947,-0.826720515,-0.7820062971 H,5.972514591,-1.2558359709,-1.744194741 C, $6.6054291154,-0.9002711024,0.2890326595$ H,7.570695687,-1.3816462505,0.1524951512 C,6.2634162555,-0.3646699279,1.5355545974
H,6.9524993308,-0.4303830187,2.3740233794 C, $5.0182628043,0.2471117175,1.6871954137$ C,4.3982655905,0.839081868,2.875584535 C,4.8827326321,0.9496789424,4.179750536 H, $5.8773617501,0.5903608633,4.4323740924$ C, $4.0624449807,1.5211845875,5.1586590236$ H,4.4225163511,1.6141897404,6.1803143557 C, 2.7741626756,1.9649850475,4.8290612499 H,2.1382928778,2.3961365368,5.598399131 C, $2.2888853049,1.8606159465,3.5190537475$ H,1.2882022516,2.2079174939,3.2945450709 C,3.1052964444,1.3045224806,2.5277606093 C, $1.5658036167,1.0551683004,-1.0955767779$ C, $2.155237929,1.8196677562,-2.0823047698$ $\mathrm{H}, 2.8914026785,2.5722465706,-1.8097171415$ C, $1.8123198809,1.6535145437,-3.4493603906$ C, $2.4169755154,2.4197327223,-4.4848474485$ H,3.1786299845,3.1502031997,-4.2208513316 C, $2.0415508238,2.2404296696,-5.8005223358$ H, 2.5088460263,2.830641059,-6.5849026834 C, $1.0437520367,1.2884839452,-6.1406491833$ H, $0.7570968485,1.1600752989,-7.1813841377$ C, $0.4399604986,0.5308639832,-5.1585151338$ H, $-0.3239480162,-0.200622406,-5.4130930307$ C, $0.8082456182,0.6901006276,-3.7930401402$ C, $0.2199325458,-0.0859327874,-2.7614662059$ $\mathrm{H},-0.5260583215,-0.8267314427,-3.0353916452$ C, $0.5827943463,0.0671636881,-1.4347625849$ C, $-0.0020705307,-0.7940501356,-0.3610934831$ C, $0.0654690276,-2.2430601998,-0.498579998$ C, $0.7822778248,-2.8582501085,-1.5581183294$ H, 1.3514917099,-2.2445209394,-2.2452071981 C, $0.7784844112,-4.229705443,-1.7326994754$ $\mathrm{H}, 1.3377784819,-4.6730401682,-2.5524471785$ C, $0.0478482616,-5.0448082438,-0.8485479388$ H, $0.019341685,-6.1218047476,-0.9928043845$ C,-0.6155745849,-4.47566462,0.224046212 H,-1.1459118157,-5.1296135969,0.9067927518 C,-0.5999961821,-3.0767490772,0.4495307719 C,-1.1954833861,-2.4792692752,1.6365409369 C,-1.7949062198,-3.2653201745,2.6521616817 H,-1.8728652537,-4.3395568616,2.5310920841 C,-2.2731230708,-2.6996204533,3.8209372044 H,-2.722385081,-3.3310103117,4.58323402 C,-2.1504251917,-1.3133439529,4.0294566621 H,-2.487100698,-0.866463259,4.9613057128 C,-1.5921686336,-0.5175289024,3.0458388397 H,-1.4933129926,0.5457014825,3.2260470186 C,-1.1320961697,-1.0657843125,1.81908179 C, $-0.5899857991,-0.222925735,0.7613325637$ C,- $-0.6552007077,1.2625830611,0.9165494945$ C,-1.8522962728,1.9102744642,1.1647033274 H,-2.7763213579,1.3393396283,1.2108988844 C, $-1.9134727677,3.3108291537,1.3835733082$ C, $-3.1409323425,3.979885015,1.6507537647$ H,-4.060807735,3.3993834641,1.6713776723 C,-3.1627366086,5.3395907521,1.8832453802 H,-4.1044355356,5.8422959055,2.0888850133 C,-1.9581343684,6.0914365388,1.8557611709 H,-1.9901262792,7.1621906127,2.041101446 C, $-0.7534698012,5.4714832907,1.5940329148$ $\mathrm{H}, 0.1705423756,6.0450398068,1.5694403656$ C,- $-0.6982625148,4.0705862667,1.3511784292$ C, $0.517735662,3.3986494228,1.0605103777$ H, $1.4382054665,3.9743038744,0.9963257795$ C, $0.551000304,2.0353405626,0.8471097292$ H,-2.1802507549,-0.5486091429,-0.7235230019 S,-3.6252571358,-1.426160652,-2.1116151233 O,-2.5395138155,-2.2596564496,-2.6103754579 O,-4.5721614484,-0.7639490455,-2.9935868093 
C,-4.6433077263,-2.4914844463,-0.9339747403 F,-3.8605528308,-2.9729396183,0.0332444509 F,-5.6228704913,-1.7613201351,-0.3952058584 F,-5.1703015684,-3.5042668967,-1.6297142388 O,-3.0802125425,-0.3202352629,-1.0847493298

\section{P2-TS1 \\ 01}

C, $-0.4000244417,-1.4172917825,-0.2136353158$ C, $-0.5443229654,-0.8185888624,-1.4295388264$ C, $0.5389262133,-0.3419303719,-2.3441448767$ C, $1.9156776738,-0.1542329958,-2.1680597902$ H,2.3989992434,-0.3628642264,-1.2230207507 C, 2.6885957923,0.3373394988,-3.2273839447 H,3.7558358619,0.4833507124,-3.0806837692 C, $2.1059009658,0.6472334719,-4.4636308836$ H,2.7236287284,1.0188508474,-5.2774008398 C, $0.726033881,0.5058637194,-4.641769288$ $\mathrm{H}, 0.2573568437,0.7809821946,-5.583355097$ C, $-0.0457463455,0.0317747079,-3.5803170363$ C, $-1.5001782541,-0.0833707792,-3.465101081$ C,-2.4932739957,0.2479239591,-4.3876410438 $\mathrm{H},-2.2272126293,0.5791883048,-5.3883506845$ C,-3.8331300689,0.1732882554,-3.993685535 $\mathrm{H},-4.621236697,0.4310855766,-4.6967670765$ C,-4.1591660297,-0.2059948471,-2.6845714254 H,-5.1999284174,-0.227357669,-2.3712456011 C,-3.1625788621,-0.5551056071,-1.764724196 $\mathrm{H},-3.4525660439,-0.823827247,-0.7577865306$ C, $-1.8183143153,-0.5266738316,-2.1566100238$ C, $0.8811437608,-1.6866673344,0.4931209427$ C, $1.9095942044,-2.4463310043,-0.0148209088$ H, $1.8572738423,-2.8094608804,-1.0372431126$ C,3.0457776136,-2.778495153,0.7743908498 C,4.1149806344,-3.5584694603,0.260584635 H,4.0678490409,-3.900389427,-0.7706988295 C,5.1970915396,-3.8769086357,1.058733232 H,6.011409034,-4.4733197056,0.6553917998 C,5.2558882239,-3.4343682916,2.4051775978 H,6.1128369712,-3.6964315302,3.0199516324 C,4.2298647805,-2.6780349426,2.9346930701 H,4.265434991,-2.3361831822,3.9664326105 C,3.1071364955,-2.3290959642,2.1352993436 C,2.0415364314,-1.5491804417,2.650086845 H,2.0643579189,-1.252306323,3.6943607325 C, $0.9559048089,-1.2223714694,1.8550671686$ C, $-0.2529645311,-0.5973921413,2.402319183$ C,0.8541108739,1.6366845187,3.1085017899 C. $2.219331885,1.5606892437,2.8202602552$ H,2.71034678,0.6252059158,2.5980396687 C,2.9939514597,2.7283872671,2.8136253508 H,4.0531581045,2.6551135308,2.5806916827 C, 2.4279672019,3.973196852,3.1112037173 H,3.0456882832,4.8676420302,3.1103525908 C, $1.0673943695,4.0615811687,3.4115260925$ H,0.609928469,5.0205539979,3.6408226762 C,0.2938367493,2.8982536092,3.4070695146 C,-1.1341382075,2.752491912,3.6628251101 C,-2.0649003533,3.7401648825,3.9913098233 $\mathrm{H},-1.751004082,4.7759924174,4.0914325724$ C,-3.3988200793,3.3763312744,4.1857793253 $\mathrm{H},-4.1376888159,4.1303179677,4.4450644741$ C,-3.7800133288,2.0365817923,4.0508707033 H,-4.8165523356,1.7486641061,4.2064414416 C,-2.8423894339,1.0457497667,3.7329327889 H,-3.1882151225,0.0267287648,3.66212053 C, $-1.5053049303,1.3958094681,3.5246216275$ C, $-0.2564028047,0.5836904788,3.2002868524$ C,-1.4168265426,-1.4218959141,2.0573069959
C,-2.2709192503,-1.9454413689,3.0124557989 $\mathrm{H},-2.1406068193,-1.688201568,4.0585748073$ C,-3.2656491793,-2.8908751769,2.6571702141 C,-4.1528069836,-3.4362588414,3.6251498531 $\mathrm{H},-4.058964609,-3.1185193479,4.6608512169$ C, $-5.1181700873,-4.3492532894,3.2506345674$ H,-5.7966991009,-4.7601711794,3.993487753 C,-5.2290346445,-4.7582416029,1.8970030988 H,-5.9934345287,-5.4782815376,1.6162891088 C,-4.3727868104,-4.2525797145,0.9371899946 H,-4.4541355793,-4.5699365708,-0.0998461522 C, $-3.3723242995,-3.3096454331,1.2903770662$ C, $-2.4545978433,-2.7901941059,0.3350327136$ H,-2.5124162411,-3.1415400224,-0.6913198805 C,-1.493016915,-1.8718083976,0.6877788121 H,0.0461946736,0.0271540686,4.3281173382 S,-0.3689441264,-0.7974255233,6.6617217333 O,0.2752626317,-1.7096597188,7.6154376397 $\mathrm{O},-1.7249928145,-1.1193461814,6.1902911153$ C, $-0.5645254604,0.8204086146,7.6016212345$ F,0.6274052753,1.2591615361,8.0402690155 F,-1.1026941434,1.7628674839,6.8122534084 F,-1.3700349277,0.6381255678,8.662653263 $\mathrm{O}, 0.5761628657,-0.3853098829,5.5351269704$

\section{P2-INT1}

01 C, $0.122818077,-0.873594367,-2.3261972753$ C, $0.2859954115,-0.4232304863,-3.610151746$ C, $1.5651401135,-0.2795740281,-4.3791234627$ C, $2.9138425521,-0.2622617221,-3.9992025969$ H,3.2141663968,-0.3595421839,-2.9653167572 C,3.9083965131,-0.0875933776,-4.9692296079 H,4.9504851258,-0.071647076,-4.6601805181 C,3.578671332,0.0713397839,-6.3214955064 H,4.3646165983,0.1922717037,-7.0626869254 C, 2.2365320645,0.1099157348,-6.7118510381 $\mathrm{H}, 1.9659320495,0.2827449215,-7.7504585207$ C, $1.246822755,-0.0409498172,-5.7402437602$ C,-0.2012743292,0.1286131328,-5.8607761997 C, $-0.9584283968,0.4876241176,-6.9762713584$ H,-0.4913201326,0.573149417,-7.9540935434 C, $-2.3172524667,0.770683406,-6.8075488998$ H,-2.923490389,1.0577438269,-7.6630428145 C, $-2.8881698856,0.7208107207,-5.5288676514$ H,-3.9327981768,0.9884345243,-5.3916173851 C, $-2.1316797038,0.336948452,-4.4151827172$ H,-2.6057948155,0.3338486895,-3.4434310564 C, $-0.7826220873,-0.0062614508,-4.5752382606$ C, $1.219729801,-1.1873591975,-1.3866056953$ C, $2.2365902125,-2.0717532922,-1.6373742636$ H,2.2616849825,-2.6130111853,-2.5783601974 C,3.2947937457,-2.2759081359,-0.7037962821 C,4.3495523805,-3.1838149723,-0.9538437429 H,4.3467273922,-3.7582673862,-1.8768011356 C, $5.3742637396,-3.3342858608,-0.0337027725$ H,6.1815227399,-4.0331374149,-0.2369413106 C,5.3850232746,-2.5912241009,1.1731223752 H,6.1932736134,-2.7322985925,1.8851374266 C,4.3686905,-1.6995161362,1.4475669848 H,4.3588059694,-1.1382589861,2.3782719055 C, $3.3061736715,-1.5176577928,0.516274541$ C, $2.2454633161,-0.6290772506,0.7806424424$ H,2.2419658905,-0.107532665,1.7312352329 C, $1.1690200878,-0.5000120954,-0.1103734659$ C, $-0.0416455848,0.1460353474,0.3040317236$ C, $-0.1942419716,2.5432445044,0.3363910958$ C, $0.488757226,2.9121141218,-0.8186819688$ H,1.3215195761,2.3198074689,-1.1885795704 
C, $0.0759418225,4.0662195589,-1.4984646469$ $\mathrm{H}, 0.5980298537,4.3744496629,-2.4004252412$ C,-1.0061520341,4.82281834,-1.0252353649 $\mathrm{H},-1.314980857,5.7135677921,-1.5664622745$ C,-1.6924785717,4.4480471314,0.1362258047 $\mathrm{H},-2.5260166635,5.0418819668,0.5027273684$ C,-1.2867030689,3.2967247486,0.8163110194 C,-1.7911515946,2.6735231078,2.0439873873 C,-2.8274761775,3.0512687426,2.9037108295 $\mathrm{H},-3.4219913285,3.9386125203,2.7014913566$ C,-3.0789904175,2.2660529689,4.0339288335 H,-3.8797328476,2.545003417,4.7143906446 C,-2.2985889533,1.1320574675,4.3103740846 H,-2.4944298528,0.5486875841,5.2063877419 C,-1.2606299033,0.7496113636,3.4505290633 H,-0.6471991949,-0.1195723976,3.6688925463 C, $-1.0294711588,1.5180989623,2.3142501622$ C, $0.0315795464,1.3232092432,1.2456012075$ C, $-1.2567437361,-0.3328179919,-0.3123654514$ C, $-2.4913685362,-0.3194578891,0.3460684623$ $\mathrm{H},-2.585776842,0.1381227784,1.320053923$ C,-3.6223977999,-0.9717794266,-0.1861353823 C,-4.8689714187,-0.9722854916,0.5019559187 $\mathrm{H},-4.9460776002,-0.4415307051,1.4478393021$ C, $-5.9554186734,-1.6354441401,-0.0293433746$ $\mathrm{H},-6.9074268217,-1.6325295347,0.4942440765$ C, $-5.8345719605,-2.3296402012,-1.2605606053$ H,-6.698505017,-2.8488444031,-1.6672144353 C,-4.6332096875,-2.3529640184,-1.9467355749 $\mathrm{H},-4.5455590638,-2.8850439262,-2.8906909739$ C,-3.5023008063,-1.6745891652,-1.4303653817 C,-2.255856171,-1.6470658336,-2.1151173464 $\mathrm{H},-2.1781617137,-2.1501410509,-3.0742576287$ C,-1.1703513461,-0.9701899058,-1.6148457462 H,1.0179695494,1.3734679953,1.7095490946 S, $2.351832354,-0.3163105719,4.6655606479$ O,3.6972516823,-0.8648234288,4.9545478085 $\mathrm{O}, 1.2871882719,-1.3234722542,4.438727471$ C, $1.8490381764,0.4789597057,6.2967238191$ F, 2.7261389533,1.4375336021,6.6622441527 F,0.6296591128,1.048732146,6.2057053934 F, $1.8026041743,-0.4366831569,7.2879152793$ $\mathrm{O}, 2.3328611523,0.8304303877,3.7203433079$

\section{P2-TS2}

01

C, $-0.8181249204,-0.2130028642,0.7824817171$ C,-1.5610523244,-1.3220620195,0.4998358776 C, $-1.1150498448,-2.7483511538,0.5771628669$ C, $0.1349833527,-3.3427543943,0.7970218382$ H, $1.0205748272,-2.7487940099,0.9759540441$ C, $0.2554720624,-4.7373753153,0.7674957976$ H, $1.2302858913,-5.1877464006,0.9378657814$ C, $-0.8558038289,-5.5543393222,0.5187151448$ $\mathrm{H},-0.7449514135,-6.6358039558,0.5096748709$ C, $-2.1017372664,-4.9766705925,0.2567306423$ $\mathrm{H},-2.9632248855,-5.5986970464,0.0259788312$ C,-2.2169384448,-3.5857821098,0.2702385311 C, $-3.3571763539,-2.7452631914,-0.0926525068$ C,-4.6236624084,-3.1206430723,-0.5428217173 $\mathrm{H},-4.901615784,-4.1705612998,-0.5906310074$ C, $-5.5166988565,-2.127345486,-0.9556257993$ H,-6.5070866665,-2.3995182888,-1.311917953 C, $-5.1253371258,-0.7821337051,-0.9312559759$ $\mathrm{H},-5.8093276616,-0.0131582951,-1.2816231657$ C,-3.8598958629,-0.4056084123,-0.4643618327 $\mathrm{H},-3.5952776484,0.6421630821,-0.4778934999$ C, $-2.9648165073,-1.3845871284,-0.0124912174$ C, $0.5944377022,-0.2150810363,1.2420805644$
C, $0.9983609263,-0.7995060319,2.4245253365$ $\mathrm{H}, 0.28524485,-1.374441797,3.0092040575$ C, $2.3310589166,-0.6703846543,2.8931716972$ C, $2.7757897869,-1.2885594087,4.0948779193$ H,2.0716328832,-1.8869048663,4.6686885716 C,4.0784093594,-1.1321089557,4.5228108383 H,4.4107890047,-1.6091753639,5.4412968615 C,4.9918047515,-0.3478593547,3.770603966 H,6.0143403805,-0.2317836719,4.1207511655 C,4.5877913512,0.2654561542,2.6018252032 H,5.2849293843,0.8671118643,2.0227048961 C, $3.2535729968,0.1189563655,2.1328882431$ C, $2.807048758,0.7464681572,0.9368279442$ H,3.5152204403,1.3662942071,0.3993659846 C, $1.5156827597,0.5825238822,0.4880133165$ C, $1.7816752458,2.002243141,-1.6618273649$ C,3.0494629874,1.4428086501,-2.0583716702 C, $4.1987748094,2.2315574816,-2.1870578591$ H,4.1347250984,3.3103048691,-2.0791625828 C, 5.4319403466,1.5960654281,-2.3897266075 H,6.338509209,2.1891365385,-2.4636068381 C, $5.4862708877,0.2081140705,-2.5171474781$ H,6.4411869098,-0.280498403,-2.6894964506 C,4.312986608,-0.5736342559,-2.479440064 H,4.3736043979,-1.6431081176,-2.6578295468 C,3.0901064959,0.0376501827,-2.2520319918 C, $1.7406840115,-0.5658597259,-2.3827376814$ C, $1.4692545034,-1.7276060169,-3.0954984899$ H,2.283980123,-2.3535460656,-3.4475871179 C, $0.1424642962,-2.0620648117,-3.4263779586$ H,-0.0528992219,-2.9704942934,-3.9892964336 C,-0.9068346957,-1.2110681555,-3.0897395929 H,-1.9251341561,-1.4402486404,-3.3885722575 C,-0.6535176974,-0.0526618744,-2.3426664628 H,-1.4742089178,0.5885077919,-2.0449852931 C, $0.6507129302,0.2217531444,-1.930140008$ C, $0.9185220566,1.2959717158,-0.733799496$ C, $-0.3518023877,1.9903393286,-0.2619374832$ C, $-0.6573673294,3.3067549271,-0.5207508564$ $\mathrm{H},-0.0225575635,3.9254216809,-1.145494657$ C, $-1.8187359983,3.912382776,0.0346199436$ C, $-2.1635018698,5.2612523426,-0.2530195987$ H,-1.5417079613,5.8235120905,-0.9456032761 C,-3.2832170887,5.8314865865,0.3190192584 $\mathrm{H},-3.5451617686,6.8614793645,0.0905232485$ C,-4.101781648,5.079477537,1.2016126071 $\mathrm{H},-4.9797196363,5.5430819301,1.6446318817$ C,-3.7942585224,3.7653562136,1.4926645627 H,-4.4243803229,3.183317891,2.1616544884 C,-2.6520391447,3.1473384869,0.9133372852 C, $-2.3201471786,1.7883203368,1.1593656421$ H,-2.9551120108,1.2005638568, 1.8168229497 C, $-1.2287220752,1.1994146815,0.5584353471$ H,1.4700013481,2.9522324055,-2.0991701515 S, $0.4655582664,5.3621941982,-3.8706135885$ $\mathrm{O}, 1.172842738,5.9926722982,-5.0070366656$ O,-0.763732994,6.043937233,-3.4065650668 C, $-0.1892348278,3.7562738059,-4.6133292078$ F, $0.825456321,2.9587833237,-5.0145411549$ F,-0.9193555789,3.0628961157,-3.7128513685 F,-0.9738220889,3.9971828627,-5.6827187714 O, 1.3502760128,4.8694420648,-2.7777726958

\section{P2-INT2}

01 C, $-1.2553007452,0.0578404406,-0.6040256183$ C, $-2.4770776059,0.0983043654,0.0101706518$ C, $-3.264144676,1.3099814073,0.4093132208$ C,-2.9352881705,2.6675992045,0.526014723 
$\mathrm{H},-1.9410037279,3.0302800081,0.3056461127$ C,-3.9022042532,3.5837012286,0.9579203935 $\mathrm{H},-3.6334690608,4.6336084434,1.0442576204$ C, $-5.2004824191,3.1669543637,1.281184981$ H,-5.9420770998,3.8945673833,1.6014677377 C, $-5.5304479899,1.8098348367,1.2234156136$ H,-6.519190226,1.4661668933,1.5172867749 C,-4.5591513105,0.8953953374,0.8123659537 C,-4.6020122786,-0.5660149782,0.8216269667 C,-5.6238850684,-1.4171982822,1.2461129401 H, $-6.5904818588,-1.0132080604,1.5368956053$ C, $-5.372569764,-2.7901049595,1.3233008855$ $\mathrm{H},-6.1541038982,-3.4690834804,1.655227304$ C,-4.1009376347,-3.2856115769,1.0050594614 $\mathrm{H},-3.8926235893,-4.3477553182,1.1073162437$ C,-3.0838481611,-2.4328619767,0.5591364309 $\mathrm{H},-2.1122693288,-2.8552463008,0.3438477183$ C, $-3.3341179457,-1.0603306527,0.4228793078$ C, $-0.4243460588,1.2445727805,-0.9197110287$ C,-0.8730129157,2.2992072541,-1.690349567 $\mathrm{H},-1.8767871986,2.2674651336,-2.1043522007$ C, $-0.0736786507,3.4483469714,-1.915096674$ C, $-0.53205721,4.5478307307,-2.6912834867$ $\mathrm{H},-1.5160782282,4.4916063578,-3.1511446377$ C, $0.2592705227,5.6668306087,-2.8545549766$ $\mathrm{H},-0.0965152814,6.5026614905,-3.4515896816$ C, $1.5405094014,5.7352232609,-2.2483155521$ H,2.1544152748,6.6210109718,-2.3898563033 C,2.0115003298,4.6825906864,-1.489827345 $\mathrm{H}, 2.99832732,4.7262640493,-1.0345859679$ C, $1.2208790323,3.5166112852,-1.3060892459$ C,1.6930632422,2.3907050332,-0.5810618217 H,2.6991125116,2.4304280165,-0.1700816705 C, $0.9174388485,1.2575985954,-0.4301527848$ C, $2.8881577668,-0.0711355952,-0.4890536742$ C,4.0846991717,-0.1127481411,0.2005650817 C, $5.30809471,-0.1305017268,-0.545331539$ H,5.2523905099,-0.0958330551,-1.6308032029 C, $6.5153198368,-0.1740109787,0.113487789$ H,7.4509492456,-0.1860559308,-0.436513214 C,6.5188281782,-0.1977421048, 1.524333133 H, 7.4687763659,-0.2306002025,2.0517486761 C, $5.3409033528,-0.1765989757,2.2725238617$ H,5.4213876138,-0.1927953313,3.3525609307 C,4.0886016813,-0.1322434315,1.6451423902 C, $2.8165295327,-0.1021850724,2.3768280497$ C, $2.7874753175,-0.1170547247,3.7907130432$ H,3.7123474392,-0.1527582083,4.355026244 C, $1.5869743029,-0.0866622575,4.4879214118$ H, 1.5919814627,-0.0990990163,5.5742399062 C, $0.3769082111,-0.039955105,3.7845733663$ $\mathrm{H},-0.5696014488,-0.0145237684,4.3176909103$ C, $0.3850022828,-0.0247263559,2.3916668178$ H, $-0.5564855524,0.0129273427,1.8554719077$ C, $1.58633333973,-0.0557999591,1.67559174$ C, $1.5472123448,-0.0393255235,0.1513546761$ C, $0.8367588012,-1.2838895799,-0.4504214067$ C, $1.5379350738,-2.4620730361,-0.6219603611$ H, $2.5449827138,-2.5682286417,-0.226102133$ C, $0.9826980335,-3.5546008276,-1.3402058712$ C,1.6932998918,-4.7698374257,-1.5371870129 H,2.683153072,-4.8775678721,-1.0992447036 C, $1.1378248655,-5.7927869909,-2.2793478876$ H,1.688057641,-6.7185053683,-2.4272611305 C, $-0.1506679345,-5.6444363724,-2.8560813164$ $\mathrm{H},-0.5745946366,-6.4587161294,-3.438164966$ C, $-0.8640527031,-4.4759327037,-2.6810485203$ H,-1.853333409,-4.3588266204,-3.1174942462 C,-0.318677721,-3.4052592543,-1.920369019 C,-1.0367293501,-2.2057581214,-1.6846289214
$\mathrm{H},-2.0425720253,-2.1089526852,-2.0832110465$ C, $-0.5081988926,-1.1823853018,-0.9219884234$ $\mathrm{H}, 2.8864279336,-0.0481767548,-1.5771516189$ S,3.6259604499,1.0981993642,-4.2336089778 O,4.9084097319,0.4824794718,-3.8046546698 O,3.641692907,2.5727008419,-4.359522019 C,3.4560624461,0.5154883083,-6.0162784141 F,3.4164967955,-0.8326257952,-6.0876046659 F,2.3242167574,0.9902097959,-6.5775335311 F,4.4961546801,0.9358947178,-6.768055952 O,2.4211134878,0.5234120207,-3.584879975

\section{P2-TS3}

01

C,-1.8286119366,-1.6484644449,-3.6341668064 C,-1.6853714605,-2.9991992653,-3.7398654544 C, $-2.5146047211,-3.9417381519,-4.5540501669$ C,-3.7080039929,-3.7773144598,-5.2695472996 H,-4.2020464465,-2.8172886202,-5.3371524348 C,-4.2924423908,-4.88003394,-5.904368375 H,-5.2172678538,-4.7421542547,-6.4589353402 C,-3.7063083371,-6.1507927978,-5.8305629225 $\mathrm{H},-4.1699927113,-6.9932923665,-6.3375021045$ C,-2.5404631632,-6.3405168073,-5.0822012342 H,-2.1006592742,-7.3297350053,-4.9829928811 C, $-1.96572033,-5.2432376022,-4.4390927803$ C, $-0.8391473438,-5.1963080545,-3.5063029956$ C,-0.0306291726,-6.2326107529,-3.0383586593 H,-0.1521920161,-7.242362731,-3.4226859824 C,0.9228433192,-5.9544576656,-2.0536669458 H, 1.5596633754,-6.7495688543,-1.6740209343 C, $1.0445369817,-4.655533284,-1.5430669906$ H, $1.7698240441,-4.4477896468,-0.760324852$ C, $0.2454994021,-3.61145822,-2.0264411188$ $\mathrm{H}, 0.3646795718,-2.6248790668,-1.6004018472$ C,-0.6951881816,-3.8673803771,-3.0323967653 C,-2.7872587634,-0.8528979609,-4.4477569341 C,-2.6912473684,-0.8998695322,-5.8302718771 H,-1.9286505862,-1.5382274355,-6.2680236342 C,-3.5760960586,-0.2040238124,-6.6868853897 C,-3.4736381615,-0.2704636766,-8.1038849248 H,-2.679483798,-0.8663955606,-8.5470860854 C,-4.3706883734,0.4136462246,-8.8979606733 $\mathrm{H},-4.2880792823,0.3616058614,-9.9804359651$ C,-5.4041515851,1.1915474535,-8.312880032 H,-6.0987472019, 1.7285138648,-8.9535380903 C,-5.5282472228,1.2726559806,-6.9409009924 H,-6.3113846508, 1.8742875811,-6.4862584049 C,-4.6210595207,0.5726499283,-6.1013213424 C, $-4.7049527554,0.6650279306,-4.6819035863$ $\mathrm{H},-5.471130701,1.3112397548,-4.2780773934$ C,-3.7927551862,0.0032466201,-3.9068821423 C, $-4.189035579,0.9675043018,-2.0959322765$ C,-5.6024974025,1.001641836,-1.8554298149 C,-6.3026403366,2.2120835052,-2.0474188844 H,-5.7500723967,3.0896430767,-2.3708432641 C,-7.6746035444,2.2594263584,-1.8492824958 H,-8.2179311349,3.1889681244,-1.9922122812 C,-8.3559379975,1.0894431609,-1.4742787627 H,-9.4326422456,1.1161786936,-1.3281756303 C,-7.6745496797,-0.1090968737,-1.2817692424 H,-8.2411203324,-0.9849351867,-0.9869665081 C,-6.2792358651,-0.1853032227,-1.4584908889 C, $-5.5021866369,-1.4059865657,-1.2090009883$ C,-6.1110137277,-2.5988160966,-0.7692193006 H,-7.1884228171,-2.6532549697,-0.6636820271 C,-5.3544570176,-3.718515622,-0.4442070846 $\mathrm{H},-5.8543325333,-4.6243851703,-0.1119688306$ C,-3.9542150609,-3.6746200821,-0.5169640209 
$\mathrm{H},-3.358682753,-4.5373294425,-0.2328225189$ C,-3.3290783484,-2.5182667863,-0.9578346993 $\mathrm{H},-2.247854036,-2.4829698502,-1.0009921396$ C, $-4.0813291055,-1.3922327243,-1.3476364791$ C,-3.4191448231,-0.188172829,-1.8461767692 C,-1.9387288168,-0.0014272417,-1.8104482844 C,-1.3919782646,0.9622075508,-0.9874136999 $\mathrm{H},-2.029896964,1.5497434807,-0.3331410585$ C, $0.0054327376,1.209209013,-0.9902934878$ C, $0.5960640183,2.1902203685,-0.147519789$ H, $-0.0424394134,2.7531446914,0.5291924401$ C, $1.9535992692,2.4302970308,-0.1978081486$ H, 2.3972240982,3.1854847932,0.4458122375 C, $2.778554742,1.6996474255,-1.0927048936$ H,3.8463322475,1.9012861753,-1.1224248221 C, $2.2337902417,0.7426321605,-1.9241356147$ H, 2.8626881673,0.1847087734,-2.6141892379 C, $0.8377263962,0.4716465011,-1.895927214$ C, $0.2426971502,-0.4962396032,-2.7476564557$ $\mathrm{H}, 0.8675968717,-1.0329266178,-3.4563061403$ C,-1.109215505,-0.7591659241,-2.6915625184 H,-3.6716091075,1.8994160204,-2.3004773775 S,-2.8595949201,4.3364443009,-3.5098589366 $\mathrm{O},-2.5694711781,3.8836194178,-2.1251910953$ O,-2.6329411139,5.7750086717,-3.7752018396 C,-1.5011507795,3.4992477952,-4.5127419904 F,-1.5305398323,2.160368594,-4.3562226368 F,- $1.6376005317,3.7621327998,-5.8305347438$ F,-0.2798754567,3.9304344099,-4.1311764471 O,-4.101252399,3.7624130551,-4.0861657697

\section{P2-INT3}

01

C,- $-0.0423115636,0.4738308716,-1.0943165145$ C, $0.0052254013,-0.6898931828,-1.786116979$ C, $-1.1479686994,-1.526489994,-2.2236959722$ C,-2.5303198477,-1.352229156,-2.1013209762 H,-2.9470071032,-0.4669099814,-1.6378527508 C,-3.3911046241,-2.3463137424,-2.5844202811 $\mathrm{H},-4.4655053601,-2.2124641982,-2.4864260354$ C,-2.8863498751,-3.5058883994,-3.1885402815 H,-3.5709145731,-4.2651734489, -3.5584621558 C,-1.5049306665,-3.6926136567,-3.3140931751 $\mathrm{H},-1.1079901276,-4.5936343171,-3.7751225029$ C,-0.6465684134,-2.705114844,-2.8302313775 C, $0.8185108002,-2.6578687863,-2.8065157471$ C, $1.7542434669,-3.585571826,-3.2640437669$ H, $1.4312018659,-4.5082490672,-3.7397462882$ C,3.1163399955,-3.3103125361,-3.0953270603 H,3.859627916,-4.022687478,-3.4446817749 C,3.5259843275,-2.1217147182,-2.4765316153 H,4.585956185,-1.9170792656,-2.3481500819 C,2.5871877599,-1.1867606171,-2.0199208972 H,2.9325795933,-0.2751242127,-1.5490191902 C, $1.2230918055,-1.4502014496,-2.1843991561$ C,-1.2606017909,1.1766150656,-0.6060528558 C, $-2.0096481988,1.9988169295,-1.4226417179$ $\mathrm{H},-1.8057078184,2.0261643338,-2.4904490764$ C, $-3.0300183054,2.8321162287,-0.8912825059$ C,-3.8286452064,3.6737714601,-1.71466064 H,-3.6629641415,3.6669902906,-2.7898397342 C, $-4.7957172677,4.4858851998,-1.1578002887$ $\mathrm{H},-5.4019290603,5.1252955023,-1.7946972921$ C, $-5.0056110549,4.4950050344,0.2464070957$ $\mathrm{H},-5.7700941707,5.1410423596,0.6706346198$ C,-4.2449358279,3.6908396942,1.0705056431 $\mathrm{H},-4.4010937945,3.6935381878,2.1468741237$ C, $-3.2425375911,2.8410007509,0.5262598255$ C,-2.4563733053,1.9875490674,1.346569756
H,-2.6393165561,1.9779105669,2.418744268 C, $-1.5016536613,1.1515471102,0.8059775167$ C, $-0.7950771691,0.1405816938,1.6970009855$ C, $-1.5975857432,-1.1027300455,2.0161359293$ C,-2.988161078,-1.1418566515,1.840908158 H,-3.5047270162,-0.2924863494,1.4119732439 C,-3.7229696379,-2.2688853127,2.1943827203 $\mathrm{H},-4.7973977842,-2.2816060028,2.0318482711$ C, $-3.0782193306,-3.3853045816,2.7446263001$ H,-3.6466300728,-4.2708961629,3.0149810182 C,-1.7053274219,-3.3607095449,2.9327151091 H,-1.2205149397,-4.2414539242,3.3379680316 C, $-0.9402602034,-2.2239794403,2.5801752807$ C, $0.5100721065,-2.2091366333,2.7340240778$ C, $1.1938083979,-3.2107558983,3.4487497398$ $\mathrm{H}, 0.6403283472,-3.9598893173,4.0015357883$ C, $2.5805341749,-3.2686605761,3.4631371335$ H,3.0726750524,-4.0583135752,4.024608951 C,3.3543875732,-2.3405519892,2.7371627097 $\mathrm{H}, 4.4362572361,-2.4281372149,2.7135908822$ C, 2.7238429776,-1.3207670113,2.0617003198 H,3.3152596251,-0.6119416931,1.4975138327 C, $1.2987154405,-1.1821729091,2.0963398468$ C, $0.6636854364,-0.064062774,1.5127775574$ C, $1.4409665058,0.9861182892,0.8346309203$ C, $2.4390555318,1.7147180763,1.4605349854$ H,2.652205722,1.5527798589,2.513575907 C,3.1702031955,2.6975334017,0.7472906516 C, $4.1880671637,3.4714805083,1.371822961$ H,4.3923846645,3.3135556084,2.4282017968 C, $4.9015456288,4.4034389453,0.647631516$ H,5.6774030404,4.9916712049,1.1305126238 C,4.6261265948,4.6025395043,-0.7304954342 H,5.1955464456,5.3413673471,-1.288576122 C,3.6416667733,3.8698076987,-1.3631108862 H,3.4279486521,4.0242852398,-2.4181461156 C, $2.8875226711,2.9032638214,-0.6450007568$ C, $1.855817261,2.1423107611,-1.262196977$ H,1.6332091576,2.3035815585,-2.314251768 C, $1.1393107064,1.2110309673,-0.5477820555$ H,-0.6962262808,0.644891272,2.7074131754 S,-1.3379901304,1.5868432786,5.4037686597 O,-0.9630333108,2.4859870828,6.5160798022 O,-2.6629136188,1.8270657562,4.786948918 C, $-1.5570509193,-0.0803703085,6.2513365201$ F,-1.9297119731,-1.0292393493,5.3690218566 F,-0.4060511379,-0.4852975065,6.8300070714 F,-2.5016287726,-0.0146699506,7.2126537459 O,-0.2432779433,1.3107334324,4.4289280903

\section{P2-TS4}

01

C, $15.6024911916,9.5245237168,18.0346480362$ C, $16.6526962813,9.1218518538,18.78810777$ C, $17.8165827996,8.3011137838,18.3481227328$ C, 18.1460035932,7.7294747361,17.1143983314 $\mathrm{H}, 17.5125611217,7.861505051,16.2463839509$ C, 19.316649529,6.9682930431,17.0023947165 $\mathrm{H}, 19.571680969,6.523635011,16.0436744895$ C,20.1586738864,6.773847826,18.1060592989 H,21.0644626841,6.1822545309,17.9984426223 C, 19.8340924112,7.3314524251,19.3477929571 H,20.4771183289,7.1739821483,20.2102264576 C, $18.6656827216,8.0857491315,19.4621557482$ C, $18.0747833811,8.7375508495,20.634653405$ C, 18.5231296447,8.7865536586,21.955080863 H,19.4578369799,8.3094384488,22.2391639251 C, $17.7448587579,9.4533288945,22.908060147$ H,18.0779641989,9.501196702,23.9418615029 
C, $16.5349033422,10.0548761055,22.535985059$ $\mathrm{H}, 15.9330396668,10.5650667481,23.283985085$ C, $16.0858286356,10.0109154435,21.2095126321$ $\mathrm{H}, 15.1475343684,10.4853642377,20.9514732007$ C, 16.8590640617,9.354204427,20.2458222806 C, $15.3846508815,9.2457262863,16.5873625175$ C, $16.046796459,9.9638272637,15.6119607228$ $\mathrm{H}, 16.8428458915,10.6471560555,15.8980060651$ C, $15.700230079,9.8426468913,14.2408658239$ C, 16.3799929193,10.5602472921,13.2175559888 H,17.2027894211,11.2160931467,13.4937195476 C, $16.0004987665,10.4260997433,11.8975533646$ $\mathrm{H}, 16.5258720857,10.9778207367,11.1220013107$ C,14.9239590235,9.5710848625,11.5415246398 $\mathrm{H}, 14.6360321662,9.4769984739,10.4975316704$ C, $14.2456013322,8.863237323,12.5124152071$ $\mathrm{H}, 13.4204833444,8.2062913815,12.2462986862$ C, $14.6150297858,8.9792618161,13.8813821816$ C, $13.9499142625,8.2503484467,14.9018488741$ $\mathrm{H}, 13.1528960524,7.5718236024,14.6124792799$ C, $14.3201608619,8.3530664028,16.2284751829$ C,13.6859553582,7.4676499511,17.2789020987 C,13.9705247214,6.0100592966,17.2060533574 C,14.6215313584,5.4381905046,16.0932603584 H,14.985539538,6.074063696,15.2970527209 C,14.8161714621,4.0696543911,15.9991764079 $\mathrm{H}, 15.3296546831,3.6567001827,15.1350056393$ C, $14.3506232778,3.2228619214,17.0185791843$ H,14.489342233,2.1475765094,16.9451982287 C, $13.7276875914,3.7635769629,18.1297728337$ H,13.3982748083,3.0923011129,18.9140403599 C, $13.5337227339,5.1606421673,18.2593909883$ C, $12.9529977806,5.7435428179,19.4631650681$ C, $12.4694826254,4.9437129787,20.5233093491$ $\mathrm{H}, 12.4548617549,3.8649297339,20.4281745743$ C, $12.0150420926,5.5047110366,21.705479283$ $\mathrm{H}, 11.6552996252,4.8571600608,22.500796602$ C,12.0393642661,6.9000702301,21.8909943029 $\mathrm{H}, 11.7203233154,7.3346872842,22.8338486645$ C, $12.4730403863,7.7132544072,20.8645627066$ $\mathrm{H}, 12.501314189,8.7845757117,21.0156161033$ C, $12.8978055292,7.1675902532,19.6189356699$ C, $13.2954322946,8.0227661747,18.5387018302$ C, $13.197621421,9.4954368018,18.6797372818$ C, $12.0060922614,10.133842269,18.9736338027$ $\mathrm{H}, 11.0907986278,9.5571761006,19.0766929049$ C,11.9523528907,11.5419051315,19.134667706 C, $10.7350928037,12.2169425232,19.4303677416$ H,9.8208904298,11.6346142706,19.5195301025 C,10.7169911682,13.5852838079,19.602270146 H,9.7834790007,14.0940452014,19.8282403314 C, $11.9149486819,14.3381492949,19.4828147887$ H,11.8855501903,15.4161757721,19.6196755063 C,13.1102163888,13.7122548383,19.192892716 H,14.0279702495,14.2881582988,19.0977928272 C, 13.1623056486,12.3028644876,19.0100134447 C, $14.3714437417,11.6264866809,18.6941068166$ H,15.2865608082,12.2016596062,18.5756336409 C, $14.3991684971,10.2593523312,18.5273972235$ $\mathrm{H}, 12.401786078,7.4822855426,16.9625189767$ $\mathrm{S}, 10.4350519252,6.6398790325,15.6185080307$ O,11.4147571158,5.8205651858,14.8897810948 O,9.3501607371,7.277038429,14.8604104669 C,9.5591767903,5.4341193716,16.7665547306 F, 10.4422314193,4.8042096822,17.5584650448 F,8.6728970668,6.0788526457,17.545291775 F,8.8999863533,4.5117140686,16.0437189106 $\mathrm{O}, 11.0801060728,7.5979959139,16.6167958229$

\section{P2-INT4}

01

C, $1.7811413356,1.3546845361,0.3497259438$ C, $2.8796969357,1.044610655,1.0775529882$ C,4.108059029,0.3448908157,0.6047620839 C,4.456251456,-0.2072913217,-0.6331757173 H,3.7796808994,-0.1683179875,-1.4776320853 C,5.7039446947,-0.826720515,-0.7820062971 H,5.972514591,-1.2558359709,-1.744194741 C,6.6054291154,-0.9002711024,0.2890326595 H, 7.570695687,-1.3816462505,0.1524951512 C,6.2634162555,-0.3646699279,1.5355545974 H,6.9524993308,-0.4303830187,2.3740233794 C,5.0182628043,0.2471117175,1.6871954137 C,4.3982655905,0.839081868,2.875584535 C,4.8827326321,0.9496789424,4.179750536 H,5.8773617501,0.5903608633,4.4323740924 C,4.0624449807,1.5211845875,5.1586590236 H,4.4225163511,1.6141897404,6.1803143557 C,2.7741626756,1.9649850475,4.8290612499 H,2.1382928778,2.3961365368,5.598399131 C,2.2888853049,1.8606159465,3.5190537475 H,1.2882022516,2.2079174939,3.2945450709 C,3.1052964444,1.3045224806,2.5277606093 C, $1.5658036167,1.0551683004,-1.0955767779$ C,2.155237929,1.8196677562,-2.0823047698 H,2.8914026785,2.5722465706,-1.8097171415 C, $1.8123198809,1.6535145437,-3.4493603906$ C,2.4169755154,2.4197327223,-4.4848474485 H,3.1786299845,3.1502031997,-4.2208513316 C,2.0415508238,2.2404296696,-5.8005223358 H,2.5088460263,2.830641059,-6.5849026834 C, $1.0437520367,1.2884839452,-6.1406491833$ H,0.7570968485,1.1600752989,-7.1813841377 C,0.4399604986,0.5308639832,-5.1585151338 H, $-0.3239480162,-0.200622406,-5.4130930307$ C, $0.8082456182,0.6901006276,-3.7930401402$ C,0.2199325458,-0.0859327874,-2.7614662059 H, $-0.5260583215,-0.8267314427,-3.0353916452$ C,0.5827943463,0.0671636881,-1.4347625849 C, $-0.0020705307,-0.7940501356,-0.3610934831$ C,0.0654690276,-2.2430601998,-0.498579998 C,0.7822778248,-2.8582501085,-1.5581183294 H, 1.3514917099,-2.2445209394,-2.2452071981 C, $0.7784844112,-4.229705443,-1.7326994754$ H, $1.3377784819,-4.6730401682,-2.5524471785$ C,0.0478482616,-5.0448082438,-0.8485479388 H,0.019341685,-6.1218047476,-0.9928043845 C,-0.6155745849,-4.47566462,0.224046212 H,-1.1459118157,-5.1296135969,0.9067927518 C,-0.5999961821,-3.0767490772,0.4495307719 C,-1.1954833861,-2.4792692752,1.6365409369 C,-1.7949062198,-3.2653201745,2.6521616817 H,-1.8728652537,-4.3395568616,2.5310920841 C,-2.2731230708,-2.6996204533,3.8209372044 H,-2.722385081,-3.3310103117,4.58323402 C,-2.1504251917,-1.3133439529,4.0294566621 H,-2.487100698,-0.866463259,4.9613057128 C,-1.5921686336,-0.5175289024,3.0458388397 H,-1.4933129926,0.5457014825,3.2260470186 C,-1.1320961697,-1.0657843125,1.81908179 C,-0.5899857991,-0.222925735,0.7613325637 C, $-0.6552007077,1.2625830611,0.9165494945$ C,-1.8522962728,1.9102744642,1.1647033274 H,-2.7763213579,1.3393396283,1.2108988844 C,-1.9134727677,3.3108291537,1.3835733082 C,-3.1409323425,3.979885015,1.6507537647 H,-4.060807735,3.3993834641,1.6713776723 C,-3.1627366086,5.3395907521,1.8832453802 H,-4.1044355356,5.8422959055,2.0888850133 
C,-1.9581343684,6.0914365388,1.8557611709 H,-1.9901262792,7.1621906127,2.041101446 C,-0.7534698012,5.4714832907,1.5940329148 $\mathrm{H}, 0.1705423756,6.0450398068,1.5694403656$ C,-0.6982625148,4.0705862667,1.3511784292 C, $0.517735662,3.3986494228,1.0605103777$ H, $1.4382054665,3.9743038744,0.9963257795$ C, $0.551000304,2.0353405626,0.8471097292$ H,-2.1802507549,-0.5486091429,-0.7235230019 S,-3.6252571358,-1.426160652,-2.1116151233 $\mathrm{O},-2.5395138155,-2.2596564496,-2.6103754579$ $\mathrm{O},-4.5721614484,-0.7639490455,-2.9935868093$ C, $-4.6433077263,-2.4914844463,-0.9339747403$ F,-3.8605528308,-2.9729396183,0.0332444509 F,-5.6228704913,-1.7613201351,-0.3952058584 F,-5.1703015684,-3.5042668967,-1.6297142388 O,-3.0802125425,-0.3202352629,-1.0847493298

\section{P'1-TS5}

01

C, $0.3371324025,-0.563359012,0.355069569$ C, $0.1904211544,0.7167351433,0.8988328098$ C, $-0.64228097,1.0956323733,2.0561734303$ C,-1.4634894249,0.332419609,2.8889674148 $\mathrm{H},-1.5419503129,-0.7410435339,2.7704810103$ C,-2.1899056787,0.9804178999,3.8979403596 $\mathrm{H},-2.8314450761,0.3968270648,4.5521683546$ C, $-2.1011108406,2.3659799761,4.0634335898$ $\mathrm{H},-2.6788145146,2.8523705146,4.8451563073$ C,-1.2711465314,3.1401743624,3.233416653 $\mathrm{H},-1.2034903913,4.216230489,3.368233849$ C, $-0.5418728566,2.4981337649,2.2432306718$ C, $0.4111085491,3.0280607888,1.2525692199$ C, $0.8580272681,4.3217499597,1.0273880019$ $\mathrm{H}, 0.4878295984,5.1518322818,1.6227942188$ C, $1.8065003967,4.5366801821,0.0119558862$ H, $2.168969625,5.5439130935,-0.1766871632$ C,2.2906450133,3.4720705305,-0.7540765975 H,3.0277318485,3.6550591465,-1.5309180539 C, $1.8289431118,2.1661322963,-0.5370243179$ H,2.2107910888,1.3521955825,-1.1397637274 C, $0.8817347847,1.9455954651,0.4654978057$ C,-0.7289754666,-1.6164629448,0.5499625674 C, $-0.5003889489,-2.8156342118,1.1882265902$ $\mathrm{H}, 0.4423401429,-2.9898030404,1.6983780597$ C, $-1.4726103783,-3.8515308809,1.1849393233$ C,-1.2621743816,-5.0907227338,1.8507370674 H,-0.3306625712,-5.244151505,2.391214571 C,-2.2220174894,-6.0815710339,1.8090505182 H,-2.0520765108,-7.0257186649,2.3205157416 C, $-3.4341450252,-5.8779158731,1.0980985645$ H,-4.1808410958,-6.6676453849, 1.0729714223 C,-3.6652864121,-4.6864606145,0.4414101655 $\mathrm{H},-4.5921245646,-4.526246562,-0.1052101503$ C,-2.6959002104,-3.6449981613,0.4685299137 C,-2.9060563096,-2.4020422477,-0.1847910647 H,-3.838245698,-2.251202478,-0.7235889995 C,-1.9697454974,-1.3855455847,-0.1453803186 C,-2.2309594139,-0.063599367,-0.7901162969 C,-3.4176605771,0.6860184351,-0.4099134379 C,-4.2701153274,0.2495245401,0.6396525172 $\mathrm{H},-4.0155809465,-0.6434038032,1.1964858509$ C, $-5.4111218731,0.9490924596,0.9873219492$ H,-6.0376498286,0.5928771834,1.8011132256 C,-5.7498538371,2.123928509,0.2910141175 H,-6.6517402002,2.6736395115,0.5482401308 C,-4.9172329471,2.5945556901,-0.7094146443 H,-5.1867045327,3.5176791739,-1.2096592202 C,-3.7279997903,1.912862285,-1.0708814804
C, $-2.7913108812,2.4576401792,-2.0442227523$ C, $-3.0150649245,3.7011587366,-2.6871290259$ $\mathrm{H},-3.9356371127,4.2451629044,-2.5093538653$ C,-2.0738963677,4.2626157579,-3.5324489835 H,-2.2737127865,5.2210814235,-4.0048785348 C, $-0.8520028023,3.6013476756,-3.7553929201$ $\mathrm{H},-0.0904825261,4.0528271682,-4.3862083681$ C, $-0.6164018725,2.37500859,-3.1616048458$ $\mathrm{H}, 0.3355611305,1.8875592577,-3.3293664913$ C,-1.5797556291,1.7552044565,-2.3211087438 C, $-1.3393295611,0.4548693207,-1.7161436812$ C,- $-0.1084277708,-0.3058417025,-2.088026393$ C, $0.1763184081,-0.6065470918,-3.407365591$ H,-0.519456985,-0.307366782,-4.1874691895 C, $1.3612584299,-1.2919853196,-3.7841772963$ C, $1.6644302241,-1.5879132991,-5.1426156226$ H, $0.962289165,-1.282441069,-5.915476767$ C, $2.8300761524,-2.2486055119,-5.4733787614$ H,3.0543771993,-2.4697518721,-6.51392472 C,3.7423658166,-2.6453291343,-4.4601315429 H,4.6558350536,-3.166816634,-4.7349915319 C, $3.4733715531,-2.374824263,-3.1336659345$ H,4.1671125547,-2.6803872923,-2.353481494 C, 2.2812203406,-1.6933269737,-2.762702935 C, $1.9612331205,-1.4154974241,-1.4068726148$ H,2.6359603077,-1.7673819634,-0.6338587937 C, $0.8126098426,-0.7368666232,-1.0667622328$ $\mathrm{H}, 1.3703102811,-0.8375231571,1.3356464769$ S,3.4624084847,-1.6463611935,2.3363125533 O,3.686684154,-2.6059138935,1.2494096934 $\mathrm{O}, 3.8939018831,-1.9836894445,3.6942882272$ C,4.4592095912,-0.1229156532,1.8584430572 F,4.1184076388,0.2796695409,0.6237535869 F,4.2302581732,0.8797677477,2.7178249095 F,5.7681262777,-0.4168950955,1.8718860455 O,2.026931884,-1.0778920397,2.3522001076

\section{P'1-INT5}

01

C,3.4783104187,0.6132862321,0.9462905541 C,3.6603775616,0.6077461648,-0.5372590145 C,3.7391338692,1.7778980648,-1.392691856 C,3.6188524254,3.1437522128,-1.1022708516 H,3.4390368536,3.4901748977,-0.0924817218 C,3.7478981252,4.0689932844,-2.1484353433 H,3.6586959206,5.1302460676,-1.9363973995 C,3.9794751096,3.6364583649,-3.4550811606 $\mathrm{H}, 4.0662464032,4.3668598006,-4.2550495465$ C, $4.1134193493,2.263025161,-3.7574250962$ H,4.3117242868, 1.9413138142,-4.7758063036 C,4.0034097861,1.3505719033,-2.7276145331 C,4.158331028,-0.1155917347,-2.7045646187 C, $4.4599868195,-1.0167506033,-3.705844067$ H,4.5896080075,-0.6925079731,-4.7345102276 C, $4.6146439871,-2.3777449992,-3.3598203113$ H,4.8547498316,-3.0988362925,-4.1365615329 C,4.4740334598,-2.807722193,-2.0393717011 H,4.6075801919,-3.8569965344,-1.7936617288 C,4.1515555583,-1.8974353244,-1.0222017544 H,4.0455052328,-2.2416349472,-0.0011303925 C, 3.9848628535,-0.5464723274,-1.3559351029 C, $2.5953801528,1.7300831694,1.4868185182$ C, $2.9981588553,2.5167700414,2.5423017162$ H,4.0069767745,2.4263334156,2.9375313175 C, $2.1074410311,3.4450197473,3.1467952279$ C, $2.5034064146,4.2764090697,4.2303430518$ H,3.52257513,4.2028470055,4.602363851 C, $1.606623795,5.1563119276,4.8025914252$ H, 1.9176483127,5.7866153025,5.6319750069 
C, $0.275145413,5.2427755753,4.3179684245$ $\mathrm{H},-0.4200980969,5.9392468018,4.7800373718$ C,- $-0.137860332,4.4472267235,3.2683339837$ H,-1.1571919994,4.5100193432,2.8932639367 C, $0.7640806828,3.5328221075,2.6562413233$ C, $0.3779617919,2.7124082381,1.5632389834$ $\mathrm{H},-0.6412562082,2.7873343981,1.1924686282$ C, $1.2626858914,1.8334307905,0.9653144781$ C, $0.8681664205,1.0147411223,-0.2156307786$ C, $0.3218578015,1.6722113803,-1.38868851$ C, $0.2832445247,3.0878677772,-1.4913563522$ $\mathrm{H}, 0.6930247655,3.6922799078,-0.6930531309$ C, $-0.2506030608,3.7200636296,-2.6007698157$ H, $-0.2584959027,4.8054625005,-2.653952676$ C, $-0.7711649899,2.9525635844,-3.6552782118$ $\mathrm{H},-1.2058925042,3.4382983687,-4.525091977$ C,-0.7135519254,1.5681627993,-3.5976155215 H,-1.1031391716,1.0045696458,-4.4372090626 C,- $0.1506625423,0.8945772693,-2.4890756656$ C, $0.0113822906,-0.5560082199,-2.4645976882$ C, $-0.369662595,-1.3714005298,-3.5552174208$ $\mathrm{H},-0.8462614221,-0.932316206,-4.4239480782$ C, $-0.1298627627,-2.737385448,-3.5613768085$ $\mathrm{H},-0.4315488063,-3.3339467752,-4.4184535828$ C, $0.5194223142,-3.33985762,-2.4717641341$ $\mathrm{H}, 0.7421011714,-4.4034444249,-2.4833857005$ C, $0.8900487463,-2.569534558,-1.3833395738$ H, $1.4060185307,-3.0440630798,-0.5593699807$ C, $0.6269973573,-1.1751088911,-1.3344092109$ C, $1.0150237936,-0.3734291324,-0.1881376684$ C, $1.5706090258,-1.0450515555,1.020917315$ C, $0.8889284056,-2.0662098442,1.657399285$ $\mathrm{H},-0.0920918038,-2.3657866309,1.2972725437$ C, $1.4377984089,-2.7469449799,2.776454032$ C, $0.7454261895,-3.804531274,3.4294894731$ H,-0.2393995411,-4.0905335506,3.0662164083 C, $1.3151166032,-4.4571444381,4.5040649399$ $\mathrm{H}, 0.779486646,-5.2642708487,4.9976152708$ C, $2.6008802172,-4.0797692062,4.9726142506$ H,3.0365998223,-4.600659635,5.8215547916 C,3.2956815843,-3.05578696,4.3604819527 H,4.2786537642,-2.7602163115,4.7196791502 C, $2.7344647515,-2.3647006224,3.2516082292$ C,3.4114742501,-1.2938128743,2.6070926365 H,4.3814031208,-0.9826027225,2.9879771631 C, $2.8521125479,-0.6460563474,1.5287862821$ $\mathrm{H}, 4.5008630631,0.7330835427,1.3441580873$ S, 6.7235546535, 1.0216060734,3.3925316268 O,6.5407906464,-0.3200120877,3.9947529071 O,6.1258431006,2.1473267455,4.1485067942 C, $8.5748066141,1.323267539,3.562463827$ F,9.2845563296,0.38106477,2.9059622195 F,8.9189414933,2.5259826503,3.0552849485 F, 8.9553666095,1.2981966429,4.8570581807 O,6.5161142856,1.0791382786,1.9215272991

\section{P'1-TS6}

01 C, $0.3522689726,-0.3067987599,1.1192672049$ C, $-0.1345790915,1.0661870124,1.2480745756$ C, $-1.5043701851,1.572735917,1.6792494832$ C,-2.6896372441,0.9044578659,1.9535789229 $\mathrm{H},-2.7561944238,-0.1689436144,1.8644003671$ C,-3.8110046992,1.6333196191,2.3754758126 $\mathrm{H},-4.7426649506,1.1080786922,2.5672879693$ C,-3.7347479444,3.0184846505,2.5530510274 $\mathrm{H},-4.6161617704,3.5760782878,2.8594554479$ C,-2.5067983016,3.6777833157,2.4115720916 H,-2.4118212803,4.7311791868,2.6622353074
C, $-1.3894029144,2.9409325674,2.0199822065$ C, $0.0352060776,3.2763316852,2.1415575759$ C, $0.6512817593,4.4328176204,2.6190705325$ $\mathrm{H}, 0.0682509722,5.3283622847,2.8179821528$ C, $2.0282114778,4.4047038592,2.8794936733$ H,2.5231516804,5.2950912567,3.25891187 C, $2.7610799973,3.2278587723,2.6920716862$ H,3.8192733842,3.2009314717,2.9383208064 C,2.1446605417,2.0698088017,2.1899191352 $\mathrm{H}, 2.7305859703,1.1651556553,2.0591796483$ C, $0.7891049356,2.1136321112,1.8850067283$ C, $-0.1616091506,-1.6058813827,0.7208302809$ C, $0.7659267376,-2.6251968843,0.9890235437$ H, 1.6839643184,-2.3723349116,1.5092728579 C, $0.5813048701,-3.9614507974,0.5893666462$ C, $1.5343901589,-4.9779188003,0.8805141306$ $\mathrm{H}, 2.4168455047,-4.7218766762,1.4615737403$ C, $1.3369921546,-6.261413057,0.4168790766$ H,2.0643431811,-7.0385709881,0.6349501499 C, $0.187182443,-6.5743998794,-0.3555459187$ H, $0.0480684002,-7.5904091795,-0.7160379975$ C, $-0.750422105,-5.6043102394,-0.6557816424$ H,-1.6279888443,-5.8462248048,-1.2505010721 C,-0.5762139248,-4.2765052947,-0.1877553609 C, $-1.5080915325,-3.2421431235,-0.4638170513$ H,-2.3775926962,-3.4988283221,-1.0620155434 C, $-1.3703566546,-1.9412899843,-0.0131690741$ C,-2.465633356,-0.9883889211,-0.355989348 C, $-3.847469288,-1.3539305937,-0.0558484754$ C,-4.165349207,-2.4820726896,0.7465180833 H,-3.3679441102,-3.0811388046,1.1691452626 C,-5.4744510119,-2.8308380469,1.0268195209 H,-5.6817457639,-3.6973031162,1.6495079282 C, $-6.529514911,-2.0584461415,0.508242159$ H,-7.5619177482,-2.3343727991,0.70719876 C,-6.2491088421,-0.9239721742,-0.2327357225 H,-7.079781498,-0.3262643127,-0.5901752711 C,-4.91727119,-0.5231766441,-0.5079004218 C,-4.6186728043,0.7369335572,-1.1754960236 C,-5.6318669627,1.6299665603,-1.6040258566 H,-6.6763824092,1.3625792244,-1.4936201308 C, $-5.3257299079,2.8628342369,-2.1549807229$ H,-6.126403672,3.5276465025,-2.4689817368 C,-3.9820392164,3.2590787922,-2.290773902 H,-3.7366912127,4.2360888001,-2.6990644875 C,-2.9713241919,2.4001094067,-1.8990695278 $\mathrm{H},-1.9395738133,2.716416254,-2.0021016371$ C,-3.2586412913,1.1212831833,-1.354661294 C, $-2.1963697954,0.2152358384,-0.9637262604$ C, $-0.7970520212,0.5818320561,-1.3119211569$ C, $-0.4510851127,0.5775984842,-2.6422147268$ $\mathrm{H},-1.2092835324,0.3223470409,-3.3782402555$ C, $0.8560033637,0.8998155254,-3.1029435757$ C, $1.2055271894,0.8840840622,-4.4745193037$ $\mathrm{H}, 0.4513406534,0.620764167,-5.211849613$ C, $2.4946544495,1.1996353878,-4.8653720472$ H, $2.7573667552,1.1858583982,-5.9200136348$ C, $3.4835679464,1.5388614924,-3.9064108228$ H,4.4913304914,1.7766477644,-4.2355163322 C,3.1701861976,1.5588711238,-2.5641976469 H,3.9203006483,1.8043279193,-1.8190140499 C, $1.8503586531,1.2432267862,-2.1354681829$ C, $1.4993923441,1.246987745,-0.7725734053$ $\mathrm{H}, 2.2795869713,1.4825292779,-0.0646228786$ C, $0.2024663909,0.9513213115,-0.3307260169$ H, $1.4033800182,-0.3791102182,1.3879781685$ S,4.3329769716,-1.2956572393,0.5939251964 $\mathrm{O}, 3.5184366626,-1.1668783337,-0.6345348712$ O, $5.2387033081,-2.4646586208,0.6532543887$ C,5.5262823202,0.1583630577,0.4773228747 
F, 6.2497097989,0.1048840888,-0.6601837549 F,4.862657687,1.3373671337,0.4860785502 F,6.3875240907,0.1692233331,1.5156383333 O,3.5947522037,-1.0032586995,1.8538115667

\section{P'2-TS5}

01

C, $2.4984063797,0.9778331687,-0.9871778079$ C, $1.6029708778,0.9116431471,0.1122173047$ C, $0.4374850973,-0.0759856302,0.119328984$ C, $0.329648521,-1.3910157726,-0.3307508123$ $\mathrm{H}, 1.1694681799,-1.9126591398,-0.7707559334$ C,- $-0.8965694037,-2.0571614253,-0.199450509$ $\mathrm{H},-0.9836402214,-3.0810501317,-0.553722516$ C,-1.9991265357,-1.4260402762,0.3897794508 $\mathrm{H},-2.9411377958,-1.9598146897,0.4865427482$ C, $-1.8888756918,-0.1157924926,0.8640481255$ $\mathrm{H},-2.7373964905,0.3795631418,1.3289064017$ C, $-0.6683896315,0.551053571,0.7313541004$ C, $-0.2928838183,1.9002034594,1.1548794636$ C, $-1.0524288734,2.8645385316,1.8204343134$ $\mathrm{H},-2.0890239451,2.6658076919,2.0798112128$ C,-0.4551959719,4.0842087001,2.1519187197 H, $-1.0307844769,4.8448995358,2.6732238701$ C, $0.8860245903,4.3233066006,1.8277615514$ $\mathrm{H}, 1.3505612126,5.2663148564,2.104012792$ C, $1.650565976,3.3539858395,1.1650414132$ H,2.6911881132,3.5581236885,0.9568011654 C, $1.05398518,2.1448569767,0.8082384524$ C, $2.8377965933,-0.1803589856,-1.8333202251$ C,3.5005021453,-1.2556958041,-1.264887089 H,3.6324160377,-1.3098900244,-0.1885622027 C, $4.0663794383,-2.2691961109,-2.0784089417$ C,4.7588798621,-3.3786202654,-1.5200476967 H,4.8521269099,-3.4494147451,-0.438920588 C, 5.3012324366,-4.346191802,-2.3410006704 H,5.8295246356,-5.1923127953,-1.9094347663 C,5.1770155443,-4.2424474177,-3.7508600959 H,5.6115430732,-5.0112549743,-4.3846120447 C,4.5102139242,-3.1752744438,-4.319731317 H,4.4116459406,-3.0961597099,-5.3998786436 C,3.9384399988,-2.1643952202,-3.501381816 C,3.2200092902,-1.0668766928,-4.048711495 H,3.0884781558,-1.020242103,-5.1265337399 C, $2.6657713602,-0.0832877604,-3.2551464533$ C, $1.8156713752,1.0061087865,-3.8183015273$ C, $0.6698373822,0.6609039018,-4.6450184204$ C, $0.2528664524,-0.6854107334,-4.8225257095$ $\mathrm{H}, 0.7671906951,-1.4790561019,-4.2941202064$ C, $-0.812328941,-1.0115810516,-5.6424306719$ $\mathrm{H},-1.1120690746,-2.0504877655,-5.7534677512$ C,-1.5070355538,0.0050297208,-6.3236327172 $\mathrm{H},-2.3362522284,-0.2432796883,-6.9811469996$ C, $-1.150700072,1.3287678538,-6.133602425$ H,-1.7236680411,2.0939969809,-6.644626425 C, $-0.0806838319,1.6965895811,-5.2794678781$ C, $0.2283420086,3.0895520976,-4.9838846411$ C, $-0.5179785376,4.159890194,-5.5378434023$ H,-1.3017293168,3.958137196,-6.2590948098 C,-0.2887153323,5.4737747226,-5.1679655474 H, $-0.8825051204,6.2711286281,-5.6075564526$ C, $0.6933873576,5.7686737042,-4.2042497919$ $\mathrm{H}, 0.8522862869,6.7933608871,-3.8782892281$ C, $1.455948543,4.748021816,-3.6657684872$ H,2.1991878955,4.9906296703,-2.9158528699 C, $1.2735315181,3.3957190569,-4.0606136681$ C, $2.105062505,2.3251416397,-3.5340597475$ C.3.2703967254,2.6658556135,-2.6658764951 C,4.242843345,3.5581347147,-3.0681346848
H,4.1929541523,3.9864232464,-4.0658465285 C,5.3009103,3.9617217817,-2.209029802 C,6.303738812,4.8771432102,-2.6263512639 H,6.2694170258,5.2682730471,-3.6405921174 C, 7.3020725419,5.2664284983,-1.7549210625 $\mathrm{H}, 8.0650731416,5.9680746481,-2.0821336094$ C, $7.3415780349,4.7579253951,-0.4307326847$ $\mathrm{H}, 8.1342332786,5.0740877944,0.2422962775$ C, $6.382879816,3.8644996147,0.0025150853$ H,6.4089323674,3.4677164317,1.0144758555 C, 5.3426839938,3.447865982,-0.8729346055 C, $4.3458267407,2.5272480464,-0.4620424884$ H,4.4033373049,2.0987500818,0.5340645196 C,3.3457170294,2.1317400415,-1.3338500775 H,2.3892049355,0.3896439911,0.9404625825 S,3.8342388531,0.2386823232,3.0699860109 $\mathrm{O}, 4.2649298841,1.6112589983,2.7546105883$ O,4.8187407462,-0.6753508242,3.6672426321 C, $2.5171456537,0.4512083548,4.396362016$ F, 1.5294712616,1.248768864,3.9583530289 F, 1.9876154679,-0.7379542318,4.7319593074 F,3.0559681005,1.0034406428,5.4982518905 O,3.0404930302,-0.4171992788,1.9500923274

\section{P'2-INT5}

01

C, $-0.2225745606,0.126253485,0.2928585574$ C, $-1.1052737111,0.4738635399,-0.8688566437$ C, $0.0415356893,0.632510845,-1.8943632209$ C, $0.9450406183,-0.3191314516,-2.362563492$ $\mathrm{H}, 0.8945676635,-1.3502531056,-2.0277895503$ C, $1.9256492129,0.0869896232,-3.2753390439$ H,2.6361605155,-0.6398695908,-3.6580563972 C,2.004678212,1.4257271126,-3.6842792249 $\mathrm{H}, 2.7827380712,1.7263635283,-4.381175934$ C, $1.0932623565,2.3811146421,-3.2154543448$ H,1.1480796682,3.4114308991,-3.5572184395 C, $0.1058510875,1.9834308644,-2.3130289027$ C,-1.0171390558,2.7181114312,-1.7243016396 C, $-1.4338336253,4.0421112243,-1.8993265685$ H, $-0.8619850089,4.7266927563,-2.5204136173$ C,-2.6143029998,4.4588224726,-1.2768489158 $\mathrm{H},-2.9581118082,5.4818795865,-1.4070678487$ C, $-3.3750234782,3.5651488653,-0.5053734036$ $\mathrm{H},-4.3060820901,3.9001381618,-0.0554650123$ C,-2.9525709409,2.2432308682,-0.3183758722 H, $-3.5434965405,1.5423304489,0.2639612072$ C, $-1.7636201319,1.8367231414,-0.9159037704$ C, $-0.0478358725,-1.2604966221,0.6539342$ C,-1.2331908885,-1.9676863313,0.8757137537 $\mathrm{H},-2.1869795947,-1.552290763,0.568751329$ C,-1.2337859348,-3.1662082057,1.6244083988 C,-2.4414178439,-3.8597648859,1.9134150854 H,-3.379508534,-3.4627239432, 1.5345925138 C, $-2.4128506979,-5.0174488146,2.664428802$ H,-3.3354809429,-5.5473866095,2.8842905307 C,-1.1812914801,-5.5193751523,3.1547406988 H,-1.1736417829,-6.4312598775,3.7462524628 C, $0.0080802519,-4.8639588943,2.8871269206$ $\mathrm{H}, 0.9515152601,-5.2561267809,3.2590887953$ C, $0.0113414263,-3.6750754561,2.1179440903$ C, $1.218006802,-2.9973570271,1.7738204412$ H,2.1620023048,-3.449926658,2.0653887351 C, $1.2246224337,-1.8314547324,1.0443082517$ C, $2.4751359202,-1.2646687741,0.4688881705$ C,3.33369459,-2.1171993003,-0.3416683809 C, $2.9746466347,-3.4505825468,-0.6758418702$ H, $2.0188309698,-3.8465900823,-0.3561503196$ C,3.8076594466,-4.2614951082,-1.4253658085 
H,3.4986989881,-5.27443387627,-1.6700858072 C, $5.0463569502,-3.7679696754,-1.8738344433$ H,5.7136015139,-4.4022085957,-2.4517973069 C, 5.4014254766,-2.458283697,-1.6033193627 H,6.343161712,-2.0904136906,-1.9934803777 C, $4.5567856037,-1.5943797797,-0.8618118601$ C,4.8646206761,-0.1808796877,-0.690265498 C, $6.0285716027,0.4090519944,-1.2453628862$ H,6.766391051,-0.2091863189,-1.7438875326 C,6.2492133429,1.773873564,-1.187474979 H,7.1485683385,2.1959717259,-1.6286668283 C, $5.2950597355,2.612082467,-0.5812091189$ H,5.4390023227,3.6894161998,-0.5693102343 C, $4.1671738067,2.0613442436,-0.0011981803$ $\mathrm{H}, 3.4376917327,2.7223923371,0.4505262212$ C,3.9371112099,0.6594113527,-0.0050707292 C, $2.7695147199,0.0712880103,0.6321192027$ C, $1.8816200861,0.9381300814,1.4536260269$ C, $2.4053315047,1.7076409675,2.4699628085$ H,3.456620458, 1.6039137141,2.7250180812 C, $1.6339553005,2.6700232274,3.1771111479$ C,2.1763681498,3.4523002995,4.2286619699 H,3.2129146516,3.3002083833,4.5197602246 C, $1.3963213826,4.3970857242,4.868776288$ H, 1.8192163001,4.9929734861,5.6734566 C, $0.0449692549,4.599558475,4.4869028383$ $\mathrm{H},-0.5524323513,5.3464511643,5.0027748757$ C, $-0.5098251875,3.8507649266,3.4693473661$ $\mathrm{H},-1.5449755518,3.9965469887,3.1701352199$ C, $0.2714123664,2.873958571,2.7916039132$ C,-0.2698685476,2.0809711425,1.7534313854 H,-1.3213500738,2.1904655555, 1.5262778062 C, $0.4856442186,1.1008159929,1.1109529902$ $\mathrm{H},-1.7773363146,-0.3445340559,-1.132787733$ S,-4.4267190876,-1.6927587782,-1.333175507 $\mathrm{O},-4.2709719744,-0.8899638616,-0.0921631898$ $\mathrm{O},-5.4471960094,-2.7629780568,-1.2740867793$ C,-5.1698851486,-0.4590475323,-2.5467751947 F,-4.3280114781,0.5703186204,-2.7722266004 F,-5.4304395859,-1.04569967,-3.7344045929 F,-6.3282974459, 0.0508087963,-2.0762383948 O,-3.1432742164,-2.0601689224,-1.9821950375

\section{P'2-TS6}

01

C,- $-0.1823030185,-0.3569804598,-0.8682369257$ C, $-1.2964006508,-0.4030007747,-1.8328157185$ C, $-1.3769022838,-1.506920413,-2.7446231572$ C,-2.6025643554,-2.0671698494,-3.1391189706 H,-3.5295784488,-1.6098140865,-2.813480466 C, $-2.5992577408,-3.2683286817,-3.8566708655$ H,-3.5408454091,-3.7354292467,-4.1287714997 C,-1.388496187,-3.8600256759,-4.2184825738 $\mathrm{H},-1.3889678948,-4.7902712409,-4.7798683666$ C, $-0.1565778286,-3.2439956838,-3.9117123065$ H,0.7697942436,-3.6771020801,-4.2786654237 C, $-0.1410345637,-2.0685516322,-3.1812211168$ C, $1.0145235502,-1.1517884679,-2.9995276394$ C, $2.0872975009,-1.0953230967,-3.881795533$ H, 2.2218308337,-1.8777233016,-4.6228693971 C, $2.9546816107,0.0132589019,-3.871940075$ $\mathrm{H}, 3.7896136457,0.0453876468,-4.5662361034$ C,2.7026521102,1.0922037204,-3.0288165821 H,3.327905365,1.9794603391,-3.062106573 C, $1.6374653064,1.0330695126,-2.1185305048$ H, $1.455932594,1.8640598462,-1.4475702026$ C, $0.8573832445,-0.1203485385,-2.0414923313$ C, $0.0448256815,-1.6727545263,-0.0853259323$ C, $-1.0202498675,-2.5163265445,0.1626019773$
$\mathrm{H},-1.9993096209,-2.3020437495,-0.2479212381$ C,-0.8925367604,-3.6552421439,0.9960532592 C,-1.9854523654,-4.5302861328,1.2506274214 H,-2.9462078793,-4.3128017965,0.7935632382 C, $-1.8251440143,-5.6251545393,2.073699263$ H,-2.6648793338,-6.2883445863,2.2660151462 C,-0.568751206,-5.890757146,2.682318346 $\mathrm{H},-0.4595659506,-6.7567627334,3.3305398484$ C, $0.5051484182,-5.0541429759,2.4605560732$ H, $1.4668013803,-5.2494966788,2.93028292$ C, $0.3705311184,-3.9170866139,1.6142658989$ C, $1.4381502462,-3.0185986777,1.3732625777$ H, $2.3885532325,-3.1989910451,1.8689905283$ C, $1.3058886661,-1.9141713544,0.5464055096$ C, $2.4535587975,-0.9775901286,0.3949636577$ C,3.7425528053,-1.4919462072,-0.0321125912 C, $3.8905785253,-2.8244244507,-0.5023176348$ H,3.0186380488,-3.46413147,-0.5707733148 C,5.1194975341,-3.3213730152,-0.8957243539 H, 5.2008079746,-4.343234756,-1.2576345576 C, $6.2594096676,-2.4977105745,-0.8307809872$ H,7.2316307756,-2.8833757647,-1.1271438581 C,6.1374170203,-1.1842483991,-0.4118381135 H,7.0261756618,-0.5637727746,-0.4022359643 C,4.8867280017,-0.6403703597,-0.0236034195 C,4.7235855028,0.7667916951,0.3142159635 C, $5.8159751113,1.6707783451,0.3043037241$ H,6.8211940131,1.3043789946,0.1299803232 C, 5.6380765228,3.0294922453,0.4969100399 H,6.4961426507,3.6966501083,0.4789659157 C,4.3417574689,3.5424407009,0.6887522509 H,4.1851943282,4.612640621,0.79794562 C,3.2617753258,2.6797629511,0.7388839013 H,2.27103957,3.0942603244,0.877931868 C,3.4186582842,1.2744566997,0.5965024104 C, $2.286866395,0.3614206306,0.6857058183$ C, $0.9425098634,0.9024650567,1.0587137742$ C, $0.8101735028,1.6411329662,2.2219339986$ $\mathrm{H}, 1.6751842889,1.7728515061,2.8665982312$ C,-0.4120490304,2.246559278,2.6087575453 C, $-0.5411350444,3.0076873328,3.803906092$ H,0.3271828021,3.1265992728,4.4483560629 C,-1.7496035187,3.5845128457,4.1373690917 H, $-1.8420427011,4.1647714578,5.0520415715$ C,-2.880825946,3.4219414464,3.2941686278 $\mathrm{H},-3.8281598218,3.8756049682,3.5741875843$ C,-2.7844355476,2.6872520977,2.1301040789 $\mathrm{H},-3.6510671066,2.5495398851,1.4875454151$ C, $-1.5497527883,2.0871730913,1.7584179311$ C,-1.4199751117,1.2962759391,0.5873576289 H,-2.3209231855,1.1115085701,0.0137516878 C, $-0.2244567209,0.7058957531,0.2308910031$ H,-2.0032109272,0.4168380912,-1.9048382673 S,-4.705017045,-0.9981217632, 0.3628840138 O,-5.8516595152,-0.1221209026,0.6884855207 O,-3.9757822689,-1.5789266868,1.5088108934 C, $-5.5209817546,-2.4991920669,-0.43228007$ F,-6.2385665249,-2.1469876426,-1.5207629333 F,-4.5958510183,-3.4019968747,-0.8285880799 F,-6.3535147166,-3.1161015078,0.4301199056 O,-3.8370941773,-0.4988122229,-0.7423561001

\footnotetext{
3-pre-IN

01

C, $1.4137540855,0.3333435077,0.284369764$

C, $2.73316166,0.2044655739,-0.3033322591$

C,3.7521597998,1.1591988718,-0.83925472

C,3.7777010965,2.5437807977,-1.0268074973
} 
H, 2.9350433704,3.1658292425,-0.7546342021 C,4.8992109862,3.1439282266,-1.6203696843 H,4.9051825251,4.2216862571,-1.7635542058 C,5.9955355499,2.3840301266,-2.037314239 H,6.8566911513,2.8700612584,-2.4881422136 C, 5.9657586389,0.9895570606,-1.9057302677 H,6.7921502405,0.3829187222,-2.2691781725 C,4.8493121112,0.3889273602,-1.3349873417 C,4.5043823904,-1.0274319288,-1.1888063521 C,5.1733936775,-2.1911172251,-1.4324255917 H,6.1863080287,-2.1929971907,-1.8274044791 C,4.5313372848,-3.4591224473,-1.1186849857 H,5.0984389914,-4.3717022691,-1.2883431982 C,3.2690645097,-3.5411584491,-0.6317639554 H,2.819986236,-4.5131281164,-0.4527672611 C, $2.3929326883,-2.320543392,-0.4803351001$ C, $3.1849753128,-1.060543078,-0.6210522934$ C, $0.7253449418,1.5617066312,0.6108205657$ C, $1.39732759,2.6907932523,1.0863318768$ H, $2.4741759944,2.6520312265,1.2056317741$ C, $0.7197991541,3.8397834082,1.5242207801$ C, $1.4086273005,4.9916378378,2.0154669732$ H, 2.4966775089,4.9854906393,2.0216169534 C, $0.7134658612,6.0874900664,2.4659837133$ H,1.2482051482,6.9612730437,2.8294762956 C, $-0.713168827,6.087518945,2.4659943557$ $\mathrm{H},-1.2478673124,6.9613236107,2.8294948241$ C,-1.4083813614,4.9916949391,2.0154878142 $\mathrm{H},-2.4964317315,4.9855920273,2.0216535789$ C, $-0.7196072222,3.8398126148,1.5242312773$ C,-1.3971889722,2.6908508648,1.0863501603 H,-2.4740369735,2.6521329145,1.2056659445 C, $-0.7252597833,1.56173741,0.6108269558$ C, $-1.4137239751,0.3334043909,0.2843773666$ C,-3.7520943305,1.1593580872,-0.8392526991 C,-3.7775731048,2.5439392098,-1.0268212226 $\mathrm{H},-2.9348854986,3.1659521709,-0.7546602391$ C,-4.8990583487,3.1441319792,-1.6203841283 $\mathrm{H},-4.9049809232,4.2218887507,-1.7635801711$ C,-5.9954198746,2.3842800621,-2.0373152643 $\mathrm{H},-6.8565552475,2.8703459369,-2.4881444238$ C, $-5.965705343,0.989806999,-1.9057183285$ $\mathrm{H},-6.7921254637,0.3832025683,-2.2691579761$ C,-4.8492834194,0.389132109,-1.3349748146 C,-4.5044158387,-1.0272413075,-1.1887868576 C, $-5.1734795396,-2.1908981452,-1.4323980588$ H,-6.186395401,-2.1927351435,-1.8273732641 C,-4.5314777427,-3.4589303093,-1.1186550648 $\mathrm{H},-5.0986203413,-4.3714858875,-1.2883069766$ C,-3.2692061823,-3.541019693,-0.6317400892 H,-2.8201696982,-4.5130083275,-0.4527414007 C, $-2.3930201657,-2.3204424566,-0.4803202376$ C, $-3.1850078966,-1.0604075786,-0.6210380299$ C,-2.7331382299,0.2045827255,-0.3033235005 C, $-0.7025236964,-0.8602562402,0.5644361425$ C, $-1.5136833257,-2.1415963548,0.7858149316$ C, $-0.7017732755,-3.3339530641,1.2367574057$ C,-1.3816620021,-4.4332314127,1.7957055753 H,-2.4670118859,-4.4050988561,1.8524347656 C, $-0.699939512,-5.5326586612,2.3063854491$ H,- $1.2518976056,-6.3685302449,2.7284942023$ C, $0.6997299046,-5.5326886346,2.3063815424$ $\mathrm{H}, 1.2516545443,-6.3685838419,2.7284872629$ C, $1.3814966704,-4.4332907325,1.7956975631$ H, 2.4668480567,-4.4052046913,1.8524207776 C, $0.7016519543,-3.3339834005,1.2367528228$ C, $1.5136104055,-2.1416614184,0.7858055865$ C, $0.7025040825,-0.8602864399,0.5644324263$ $\mathrm{H},-1.6839816185,-2.3599758832,-1.3365509873$ H,-2.2214742899,-1.9238392281,1.6029200585
H,2.2214153666,-1.9239348818,1.6029067475 $\mathrm{H}, 1.6838881411,-2.3600424914,-1.3365624802$

\section{5-pre-IN}

01

C, $-1.338391447,0.4597142256,0.2535277072$ C, $-2.6546628488,0.8261843442,-0.267353887$ C, $-3.5326683149,-0.1939033106,-0.5544643733$ C,-3.2296597588,-1.6586227474,-0.450111104 C, $-4.481642609,-2.4919884189,-0.5933240499$ $\mathrm{H},-4.3930145315,-3.5649906925,-0.4618673946$ C, $-5.6464775039,-1.9640821085,-1.0392069293$ H,-6.4980735037,-2.6185285609,-1.2110353697 C,-5.812999626,-0.543471672,-1.3156292443 H,-6.7715919668,-0.1845912329,-1.682555458 C,-4.7746143032,0.3074007695,-1.0874216794 C,-4.6030870834,1.7541679673,-1.2269559088 C, $-5.455292918,2.7139404249,-1.7615426585$ H,-6.4555393628,2.4402927974,-2.0897664783 C,-4.9953210348,4.0292542956,-1.9043189455 H,-5.6449442645,4.7908791484,-2.3273401096 C,-3.6852744826,4.3484667395,-1.5363091123 H,-3.3135405622,5.3585407913,-1.6905309654 C, $-2.8274152037,3.387357961,-0.9793429214$ H,-1.8086979063,3.669736901,-0.747535755 C,-3.2866972281,2.0824649038,-0.7755124972 C,-1.1052410476,-0.8824765512,0.5119302004 C,-2.3018802702,-1.8146522051,0.7754102921 C, $-1.930305642,-3.217394339,1.2317785687$ C,-2.9132807072,-4.1143032298,1.6620484641 H,-3.9553380714,-3.8082851182,1.6557501947 C,-2.5840624117,-5.3857378314,2.1443062109 H,-3.3712104951,-6.060213648,2.4709830267 C,-1.242649334,-5.772925476,2.222338093 H,-0.9755379316,-6.7586732461,2.5943931745 C, $-0.247899366,-4.874174114,1.8489428175$ $\mathrm{H}, 0.8003053817,-5.1507737447,1.9418645046$ C, $-0.5704967446,-3.5886042046,1.3655830654$ C, $0.4820038282,-2.6571709399,1.0324951583$ $\mathrm{H}, 1.5021008866,-2.9912264809,1.1782927714$ C, $0.2491147768,-1.3803285697,0.5906131515$ C, $1.3394238023,-0.4566704282,0.2557084222$ C, $3.2893627615,-2.0795369549,-0.7700510259$ C, $2.8304049967,-3.3844527216,-0.9744657452$ $\mathrm{H}, 1.8113201621,-3.6668054992,-0.744247771$ C,3.6891486626,-4.3456240724,-1.529959154 H,3.3176600632,-5.3557152834,-1.6846590135 C,4.9997790223,-4.0264531799,-1.8959205616 H,5.6500739297,-4.7881255389,-2.3178226965 C, 5.4595236845,-2.7111234037,-1.7525603005 H,6.4602909316,-2.4375126993,-2.0792236584 C,4.6064690223,-1.7512908926,-1.2194371952 C,4.77777444766,-0.3045080607,-1.0797930466 C, $5.8165215882,0.5463386213,-1.3064448823$ H,6.7756961034,0.1874168192,-1.6718059135 C,5.6495604366,1.9669801922,-1.0304477477 H,6.5014287639,2.6214072439,-1.2009954124 C, $4.4840180288,2.4949367629,-0.5864773911$ H,4.3951812234,3.5679538389,-0.4552826323 C,3.2318088599,1.6615872511,-0.4451617536 C,3.5349826336,0.1968560333,-0.5488678199 C,2.656521582,-0.8231992913,-0.2630387746 C, $1.1058629256,0.8855495927,0.513587965$ C, $2.3020816723,1.8177552907,0.7788649449$ C, $1.9297818875,3.220549173,1.2344824763$ C,2.9120716367,4.1175071421,1.6662126627 H,3.9541376899,3.8114884439,1.6616060527 C, $2.5820870277,5.38899661,2.1478015512$ H,3.368714728,6.0635096981,2.4756527802 
C, $1.2405515984,5.776193029,2.2236562064$ H, $0.9728490169,6.7619831458,2.5951738015$ C, $0.2463965411,4.8773991391,1.8487818027$ H, $-0.801954607,5.1540093268,1.9400049303$ C, $0.5697619902,3.5917742331,1.3660820093$ C,- $0.4822076473,2.6603030504,1.0314265998$ H, $-1.5025352854,2.9943752038,1.1755634092$ C,-0.2486162588,1.3834104199,0.5900608311 $\mathrm{H}, 2.8511830194,1.3610426109,1.623114103$ $\mathrm{H}, 2.6150892825,1.9238058217,-1.3367905701$ H,-2.611522969,-1.9209417351,-1.3407284297 $\mathrm{H},-2.8523233761,-1.3578437602,1.6187333865$

\section{2-TS2-T1}

21

C,-1.3375793763,-0.4007805895,-0.0917879742 C, $-2.7604185464,-0.6430814856,-0.0232567176$ C, $-3.6281514539,0.3099345602,-0.583462621$ C,-3.2508985788,1.5176445042,-1.2752152845 $\mathrm{H},-2.4753294772,1.4761463822,-2.0368707232$ C, $-4.3074365969,2.4746995643,-1.5010040422$ $\mathrm{H},-4.0816794026,3.3848569534,-2.0464504998$ C,-5.5915265578,2.2073051082,-1.0917717035 H,-6.3805035833,2.9225197288,-1.3052055793 C,-5.941794657,0.9927161936,-0.4073406139 $\mathrm{H},-6.9615808227,0.8517082492,-0.0608425656$ C,-4.9693467112,0.0540936838,-0.1634372421 C,-4.9378741649,-1.1965596604,0.6138687485 C,-5.9473079252,-1.9031650024,1.2466576142 H,-6.9822621357,-1.5797646025,1.180232446 C, $-5.5997705049,-3.0435904462,1.9964399092$ $\mathrm{H},-6.3794923796,-3.6101071847,2.4976104735$ C,-4.2654128031,-3.4450968838,2.1162263076 $\mathrm{H},-4.0161410042,-4.3177923513,2.7127878816$ C, $-3.2403137955,-2.7366139714,1.4729112612$ C,-3.5773992849,-1.6198291872,0.7036485448 C, $-0.9390282472,0.9687416386,0.0017227089$ C,-1.9175510306,2.0611792446,0.1503352538 $\mathrm{H},-2.6595971122,1.941906375,0.9347484802$ C,-1.4914174663,3.4183457731,-0.0763205409 C,-2.3434661894,4.5143536833,0.1596547929 H,-3.3401912714,4.3420795908,0.5556114831 C,-1.9188238371,5.7992271571,-0.14376527 H,-2.5836601364,6.6406979021,0.0282964382 C, $-0.6308884952,6.0280767618,-0.6846594154$ $\mathrm{H},-0.3222585511,7.0417395863,-0.9226679959$ C, $0.2375005598,4.9726006356,-0.891404461$ H, $1.2413238988,5.1390904717,-1.2719046416$ C, $-0.1671342775,3.6521647894,-0.5658407543$ C, $0.7564594213,2.5841390235,-0.57638769$ $\mathrm{H}, 1.7593032333,2.7861054633,-0.9374201263$ C, $0.4376008618,1.2852272821,-0.1607206642$ C, $1.4563994675,0.2462636441,-0.0497683942$ C, $3.5871839198,1.6761594816,0.6933199698$ C,3.2261415956,2.8977076484,1.2698314351 H, 2.1860877725,3.1665771673,1.4233158239 C, $4.230338369,3.7837873588,1.6832280834$ H,3.95086224,4.7328011954,2.1327251577 C, 5.5834591013,3.4578671856,1.5307239432 H,6.3474005264,4.1608134857,1.8519353077 C, 5.961412863,2.2199269801,0.9899840322 H,7.0110159026, 1.9513611856,0.9036297896 C,4.9684446985,1.3325563176,0.5881803679 C, 5.0646991428,-0.0475482516,0.0890792923 C,6.1147054602,-0.8613782306,-0.2817650237 H,7.1461956768,-0.5239043863,-0.2317634199 C,5.8080047711,-2.1524357016,-0.7851124866 $\mathrm{H}, 6.6193648456,-2.7840235962,-1.1368078645$ C,4.5082610289,-2.6348827343,-0.858630404
H,4.3485160153,-3.6113454944,-1.2963100252 C, $3.4073975142,-1.8388126769,-0.4219992446$ C, $3.7351096375,-0.531781106,-0.0257130484$ C, $2.7994467206,0.5122100129,0.2233517526$ C, $1.0589910913,-1.129040635,-0.2379675013$ C, $2.0002833844,-2.1893614552,-0.3729697355$ C, $1.5144097382,-3.5540878685,-0.4592334546$ C, $2.3123451712,-4.7018954397,-0.2377836185$ H,3.3286282142,-4.5994835662,0.1170706997 C, $1.7981913875,-5.9785175182,-0.4097207947$ H, $2.4381679836,-6.835554512,-0.2179687303$ C, $0.4586513079,-6.1860078951,-0.8117749813$ H, $0.0835420501,-7.1944111078,-0.9590645924$ C,-0.3723868151,-5.0984024651,-0.9584067366 $\mathrm{H},-1.4243016874,-5.2253407054,-1.2003791928$ C, $0.1218673623,-3.7786319849,-0.7411256071$ C, $-0.7749216433,-2.7043945685,-0.6803908523$ C, $-0.3586195467,-1.4197335991,-0.3175466217$ $\mathrm{H},-1.8219919759,-2.9021386485,-0.8806046139$ H,-2.2098948901,-3.0591174575,1.579793072

\section{2-TS2-T2}

21

C, $1.3076780268,-0.4575771616,0.0134076605$ C, $2.7072547411,-0.7506336817,-0.1760478431$ C, 3.6546471927,0.1168487808,0.392425086 C,3.3805180766,1.2744775399,1.2064391451 H,2.656254141,1.1982835198,2.0144469211 C,4.497503758,2.159540656,1.4406441773 H,4.352336211,3.0290805349,2.0727069151 C,5.7391673815,1.8670828093,0.9299190934 H,6.5759805957,2.5228836752,1.152226212 C, 5.9845353806,0.700885737,0.1254167125 H,6.9742935161,0.5419242725,-0.2931294729 C,4.9513155006,-0.1654861129,-0.1354979753 C, $4.8056838086,-1.3480963217,-1.0033550262$ C, $5.7306818983,-2.0501363808,-1.7583437692$ H,6.7826015912,-1.7793432767,-1.7488481717 C,5.2740981712,-3.1198920122,-2.5535083365 H,5.9870113834,-3.6816581184,-3.1503740622 C,3.917584653,-3.4587620929,-2.5956673397 H,3.5861105413,-4.2790607058,-3.2255744105 C, $2.9770253403,-2.7542175418,-1.8304595618$ C,3.4236684403,-1.7063024141,-1.0220266101 C, $0.9666601921,0.9299046726,0.0476356602$ C, $1.9828118717,1.9928794201,-0.0611626532$ H,2.6670921511,1.9241013431,-0.9025660522 C, $1.6276069326,3.3373587109,0.3190259198$ C, 2.5145888587,4.4169870548,0.1437722444 H,3.4826419069,4.2438206561,-0.3179113861 C, $2.1633792552,5.6821023026,0.5900387034$ $\mathrm{H}, 2.8568973621,6.5087200019,0.4657417579$ C, $0.9149137873,5.9078561268,1.2173139687$ $\mathrm{H}, 0.6635328727,6.9046727707,1.5674933719$ C, $0.0113660798,4.872432225,1.36736499$ H, $-0.9648377592,5.0419509226,1.81304189$ C, $0.340500147,3.5749181123,0.8984511639$ C,-0.6295409127,2.5482242435,0.8537499879 H,-1.6057344552,2.7497086838,1.2825221905 C, $-0.3865273135,1.2854869713,0.302346905$ C, $-1.4518393057,0.3020879934,0.130180463$ C,-3.5021739773,1.8570754034,-0.5889083477 C, $-3.092550662,3.1542003547,-0.9181506828$ $\mathrm{H},-2.0544496404,3.4589525492,-0.8599773941$ C,-4.0390796985,4.0853022252,-1.3671239373 H,-3.7148408973,5.0919392347,-1.6170905572 C, $-5.3870892551,3.7344132926,-1.5028952834$ H,-6.1068183944,4.4721671428,-1.8472724537 C, $-5.8078463078,2.4264969264,-1.2250729296$ 
H,-6.8454282452,2.1362276156,-1.3679721898 C, $-4.8695183335,1.4974948828,-0.7876608741$ C, $-5.0128165386,0.0573848652,-0.5419306825$ C, $-6.0653985319,-0.8241025151,-0.6730178827$ $\mathrm{H},-7.0598144068,-0.4895772615,-0.9555454837$ C, $-5.8004305984,-2.2036994754,-0.4832459573$ $\mathrm{H},-6.6006591384,-2.9202909846,-0.6475045133$ C, $-4.5449017639,-2.6769568641,-0.1285263472$ $\mathrm{H},-4.4013271645,-3.7477830993,-0.0820878895$ C, $-3.4545836147,-1.7805228137,0.0869041938$ C, $-3.7315472006,-0.4261274414,-0.1730627926$ C, $-2.7776169756,0.6352755584,-0.153019246$ C, $-1.1017510273,-1.0916007164,0.2673498896$ C,-2.0812781164,-2.1138946592,0.4150537075 C,-1.6557689888,-3.4118943454,0.9082626528 C, $-2.5264640315,-4.361136338,1.4977871328$ $\mathrm{H},-3.5659009716,-4.117685781,1.6673975702$ C, $-2.0609601843,-5.5912670406,1.9361535093$ $\mathrm{H},-2.7600693699,-6.2878064073,2.390954623$ C,-0.6971249065,-5.9467148615,1.8232358886 H, $-0.3578158622,-6.9223901466,2.1582386954$ C,0.1955921391,-5.0211030706,1.3337345539 H, 1.2602300484,-5.2366932771,1.2903097404 C, $-0.2542912514,-3.73719677,0.9058804992$ C, $0.6843169382,-2.7594272728,0.5508606108$ C,0.3034332338,-1.4459494794,0.2649636186 H, $1.7332186492,-3.0326739379,0.5781081481$ H,1.9265370259,-3.0247214084,-1.8710690581

\section{2-TS2-T3}

21

C, $1.3185829503,-0.4162253593,-0.08160153$ C, $2.7250675954,-0.5755448828,-0.3881722252$ C,3.7499026446,-1.5631472239,-0.0225709747 C,3.6949239526,-2.752038616,0.7128920707 H,2.7561307573,-3.1437547432, 1.0889120436 C,4.8874640844,-3.439114172,0.9813362575 H,4.8509198291,-4.365374566, 1.5472643888 C, 6.1167507716,-2.9468587778,0.5329363936 H,7.0284592291,-3.4983405037,0.7446424879 C,6.19099007,-1.7269540813,-0.1654541423 H,7.1516707013,-1.3240960719,-0.473472487 C,5.0159836191,-1.0408870182,-0.4258630948 C,4.7885746594,0.2933876392,-1.0040031138 C, 5.6318349336,1.3295324351,-1.3224406504 H,6.7104923038,1.2088393737,-1.2806832908 C,5.078969553,2.613959827,-1.6541726657 H,5.7605247141,3.4113549999,-1.9357539403 C,3.726770528,2.8542698911,-1.6372287893 H,3.3352120588,3.8271003772,-1.9150765533 C, 2.7942422248, 1.7948902961,-1.3278331161 C,3.3756700029,0.505757858,-1.0099673441 C,0.9245529365,0.9131392997,0.2741235124 C, $1.9398774368,1.9548408811,0.4933413274$ H,2.8525257819,1.6480306461,0.9947862001 C, $1.5268230861,3.291841825,0.8537125818$ C, $2.4584128431,4.3174042379,1.0933945028$ H,3.5171135631,4.1275703745,0.9421386176 C, $2.0236599586,5.5666865916,1.5145103472$ H,2.7473884113,6.3590840968,1.6820862754 C,0.6502085551,5.8185035315,1.741029652 H,0.3321407718,6.8018450782,2.0744966867 C, $-0.2802184122,4.8151930412,1.5515347508$ H,-1.336835542,4.9883361602,1.7365262692 C, $0.138964023,3.5365561701,1.0967609013$ C, $-0.7864505449,2.4850342221,0.9414003351$ $\mathrm{H},-1.8090755705,2.6752330992,1.2428822958$ C,-0.4520305764,1.2227368002,0.4320899594 C,-1.4785246477,0.2150236502,0.173641607
C,-3.626041988,1.711605766,-0.3754397769 C, $-3.277552641,3.0069104271,-0.7740880676$ H,-2.2426250202,3.3197381268,-0.8553981559 C,-4.2847044269,3.9201367551,-1.1142855258 $\mathrm{H},-4.0082664653,4.9260761329,-1.4185555942$ C, $-5.6342672712,3.5509078172,-1.0734956576$ H,-6.4019914142,4.2753837039,-1.3314963067 C, $-5.9983771382,2.2399530364,-0.7367799758$ H,-7.040651742, 1.9327717563,-0.7545329358 C,-4.9995037582,1.3275082482,-0.4117887849 C, $-5.0813991089,-0.1194937738,-0.1802458821$ C,-6.1238853429,-1.0209255471,-0.207186716 H,-7.1534580924,-0.7014669238,-0.3428656156 C,-5.8071863043,-2.3992849693,-0.1097321744 H,-6.6086046752,-3.1293399935,-0.1854366549 C,-4.5051071493,-2.8545258566,0.0372483199 H,-4.3432905218,-3.9223391612,0.0213450944 C,-3.410827692,-1.9415380851,0.1420145146 C,-3.7525639627,-0.5830779728,0.0066578985 C, $-2.8306344624,0.5037518526,-0.0308970684$ C,-1.0675512767,-1.1710269318,0.1436736948 C,-1.9956065423,-2.2474439993,0.2156213579 C, $-1.473056253,-3.6022523631,0.2833053628$ C,-2.1607478293,-4.7084882869,0.8310488263 H,-3.0866578252,-4.5622001628,1.3712523108 C,-1.6357314581,-5.9905677731,0.7450457397 H,-2.1856507936,-6.8171274132,1.1867960707 C,-0.3998305309,-6.2394429772,0.1055857792 H,-0.0219251629,-7.2544540546,0.0269371023 C,0.3410592984,-5.1755603795,-0.3597475014 H, 1.3303009197,-5.3221203921,-0.7857743514 C, $-0.1519367545,-3.8447738,-0.2259564871$ C, $0.7030724674,-2.7582994027,-0.4547336268$ C, $0.3374618087,-1.4560002024,-0.0981175634$ H, $1.6843501689,-2.9596909218,-0.8643614387$ H,1.8467796834,1.8390933342,-1.8601707569

\section{2-TS2-T4}

21

C, $-1.2854645209,0.5626208504,0.1681537595$ C,-2.5029852397,0.8483625332,-0.5202892084 C,-3.3708220598,-0.2355292857,-0.857046695 C, $-3.115075747,-1.6496881489,-0.8581304864$ C,-4.2436663516,-2.5254260411,-0.9277836601 H,-4.0796276364,-3.5978147435,-0.9224197086 C,-5.5081455377,-2.0053491948,-1.1000875367 H,-6.3555649806,-2.6775196364,-1.1988393018 C,-5.73602896,-0.5959628883,-1.2244035978 H,-6.7475329605,-0.2282368561,-1.3708409793 C,-4.6720481258,0.2723891655,-1.1349124584 C,-4.5482080593,1.7336807156,-1.2328038635 C,-5.4732994443,2.6816270575,-1.638927133 H,-6.5017709566,2.4012767557,-1.847743547 C,-5.0433748422,4.0108068971,-1.8021446035 $\mathrm{H},-5.7521315463,4.768274817,-2.1241209207$ C, $-3.7072167451,4.3551356505,-1.5852672192$ $\mathrm{H},-3.3767651085,5.3750822658,-1.758631271$ C,-2.7750878702,3.4014956257,-1.1500834298 H,-1.7408026983,3.6957893182,-1.0304198729 C,-3.1979208144,2.0853335587,-0.9360262802 C,-1.0651332668,-0.7963188034,0.5808130556 C,-2.1689345081,-1.6069502229,1.0806252238 C, $-1.9237762627,-2.9254365134,1.5697246752$ C,-2.9555480395,-3.7146469706,2.1180579556 H,-3.9666552794,-3.3188155968,2.1540936532 C,-2.677095601,-4.9911519421,2.5870780881 H,-3.4749692541,-5.6006568546,3.0009694052 C,-1.3615055258,-5.5006224617,2.5395782388 H,-1.1591915579,-6.4995455597,2.914605182 
C, $-0.3253382946,-4.730735398,2.031640389$ $\mathrm{H}, 0.6921245543,-5.1109950556,2.0135183331$ C, $-0.5861867282,-3.4330512353,1.5320684944$ C, $0.4634839433,-2.6003528477,1.0682294804$ H, 1.4744539678,-2.985199782,1.1422744169 C, $0.2558814105,-1.3153385247,0.5675376603$ C, $1.3572473416,-0.4429970069,0.137005913$ C,3.1277116188,-2.1191205335,-0.9234171037 C, $2.5447830742,-3.3472880082,-1.2507371543$ H, 1.4969403077,-3.5485524396,-1.0556888506 C, $3.3241284789,-4.3346455086,-1.8686876355$ H,2.8717300209,-5.2904729277,-2.1192244685 C, $4.6720180713,-4.1028633393,-2.1705692627$ H,5.2614988929,-4.8835774943,-2.6440152276 C, 5.2589273991,-2.8617103985,-1.8896911751 H,6.294153458,-2.6666173937,-2.1574390098 C,4.4866149063,-1.8743570167,-1.2838931415 C,4.8030167939,-0.4713663814,-0.9807911127 C,5.9226178891,0.3139364845,-1.1763075267 H,6.8400474972,-0.0920093104,-1.5934666686 C,5.8287042098,1.6913263546,-0.859907523 H,6.6783933036,2.3372094708,-1.064436877 C,4.6778289619,2.2541066168,-0.3232827418 $\mathrm{H}, 4.6573779697,3.3253532884,-0.1751249516$ C,3.5295541659, 1.4541851556,-0.0432773731 C,3.6302976638,0.1061790632,-0.433334882 C, $2.5841197359,-0.8656596715,-0.3475381427$ C, $1.160053275,0.9732565707,0.3741246909$ C, $2.2416619951,1.89019047,0.4691035524$ C, $1.9874267321,3.1854166699,1.0788831219$ C,2.9984305685,4.0106654196,1.6292908188 $\mathrm{H}, 4.0243003671,3.6704316039,1.6600029415$ C,2.6948409151,5.2338566155,2.2088227476 H,3.4971765527,5.8315803317,2.6329793073 C, $1.3644492636,5.701177071,2.2774242638$ H, 1.1471633383,6.6673430866,2.7234461095 C, $0.3455033772,4.8957422051,1.815222909$ H,-0.6932088293,5.2049554468,1.9012572384 C, $0.6297850775,3.6261433374,1.240353544$ C,-0.4309978387,2.7795730855,0.8582233585 $\mathrm{H},-1.4460679966,3.1336750936,0.9875138875$ C, $-0.1954148539,1.4736166169,0.4581609207$ H,-2.1718238077,-2.0262569677,-1.2459856685 H,-3.0348802494,-1.0894843078, 1.4797719341

\section{2-TS2-C1}

21

C, $-1.3806762955,0.42646024,-0.0200149539$ C, $-2.8138970005,0.4297374383,-0.0077583984$ C, $-3.4972115219,-0.6549848266,-0.6058659654$ C, $-2.895141775,-1.7903282349,-1.2574585532$ H,-2.1049741797,-1.6337589768,-1.9889821073 C, $-3.772249578,-2.9056371867,-1.5208929319$ $\mathrm{H},-3.3738516867,-3.774451388,-2.0342422849$ C,-5.1070315249,-2.8404444048,-1.1922919743 H,-5.7596350119,-3.6735263443,-1.43616985 C, $-5.6780328126,-1.6924246691,-0.5524815964$ H,-6.7269361161,-1.7053655746,-0.2702785691 C, $-4.8762380752,-0.6101065913,-0.264488859$ C, $-5.0852189233,0.6316058906,0.5013830429$ C,-6.2255675726,1.159328222,1.082790117 H,-7.1891076062,0.6712972444,0.9667914576 C,-6.105438883,2.3361360683, 1.8475126876 H,-6.9903625836,2.7656696708,2.3083868448 C,-4.8611321827,2.9459574702,2.0357060465 $\mathrm{H},-4.7862888339,3.8422090211,2.6443761863$ C,-3.704656236,2.4173824314, 1.4451215512 C, $-3.8171393865,1.268424012,0.6564502791$ C, $-0.5828291675,1.6029355828,-0.1994552459$
C, $-1.1718647785,2.8203367645,-0.5758539579$ H,-2.2464527595,2.8944122937,-0.6904778321 C, $-0.406399564,3.9555076436,-0.8727425356$ C,-1.0056080665,5.2003698907,-1.2301137631 H,-2.089786916,5.2766284503,-1.2452889626 C, $-0.2154318605,6.2825412827,-1.5444336284$ H,-0.6705072789,7.2322640833,-1.8110150991 C, $1.2025504271,6.1660797925,-1.5297731491$ H,1.8093787723,7.0304647676,-1.7855747959 C,1.8115278609,4.9721442578,-1.2005778946 H,2.8947006503,4.8839113278,-1.1961716438 C, $1.0257175259,3.8400967798,-0.8558406737$ C, $1.6102292974,2.5958076445,-0.533174686$ H,2.6845974688,2.5135814761,-0.6324356245 C,0.8563883449, 1.4899677114,-0.1669198679 C, $1.4779917517,0.1887000636,0.0587820277$ C,3.9783801405,0.8061211483,0.7643891721 C,4.0512813599,2.0308929791,1.435180683 H,3.1558988917,2.5996455861,1.664078 C,5.3042760837,2.5198142317,1.8252760116 H,5.3659313979,3.4698152854,2.3491442282 C,6.4754285146,1.8017183673,1.548611814 H, 7.43845721,2.2050132651,1.8499265382 C,6.4147397527,0.5573477023,0.9052446945 H,7.3201675083,-0.0138933007,0.7183191905 C,5.1708285814,0.0583967746,0.5309727499 C,4.7942571399,-1.238586006,-0.0519969067 C,5.5010976629,-2.3071471763,-0.5710849326 H,6.5870828533,-2.3055248771,-0.6067355495 C,4.7737521445,-3.4007327784,-1.1111218246 H,5.3207002377,-4.2125356173,-1.5819377374 C,3.3896038708,-3.4635104896,-1.0639340718 H,2.8864336869,-4.3038913981,-1.5258433112 C, $2.6378681253,-2.4012137116,-0.4755101712$ C,3.3786128955,-1.2781532442,-0.0687534249 C, $2.8346017253,-0.0102768594,0.3022868704$ C, $0.6544008647,-1.0131063649,-0.0435206057$ C, $1.2273669839,-2.3341807721,-0.1999968981$ C, $0.4106034116,-3.5183400209,0.0075407722$ C,0.9620620679,-4.8126553912,0.1956873518 H,2.0314858755,-4.9609048899,0.1762618881 C,0.1576888938,-5.9036442537,0.4831304291 H,0.6150360749,-6.8791666312,0.6213784664 C,-1.2334268089,-5.7525982681,0.6397245367 H,-1.8547302618,-6.6109083106,0.8775553324 C,-1.7914481968,-4.4914177195,0.5343512374 H,-2.8550848013,-4.3425327541,0.6975162885 C, $-0.9917330629,-3.3755380689,0.2167574317$ C,-1.5929423212,-2.0637558398,0.2313360097 C, $-0.7533226653,-0.871830963,0.0496267595$ H,-2.3666059795,-1.9780068064,0.9883760706 H,-2.745694779,2.8985988486,1.6061460708

\section{2-TS2-C2}

21

C, $1.3272570029,0.4229445637,0.0039600658$ C, $2.7393193813,0.4660443737,-0.2226632916$ C,3.5377396176,-0.5717799336,0.3024244506 C,3.0758204054,-1.6795802502,1.0963101429 H,2.396227977,-1.491446495,1.9248820756 C,4.0397972014,-2.7338098354,1.3154747065 H,3.7657089776,-3.571431751,1.947175905 C,5.3126672152,-2.6390190751,0.8028673859 H,6.0335374454,-3.4227873943,1.0158791697 C,5.7395859375,-1.5170107866,0.0185694118 H,6.7442856736,-1.505707865,-0.3943659527 C,4.8558938894,-0.4893474489,-0.224038752 C,4.905777351,0.7308711856,-1.0511178316 C,5.9295831013,1.2843021106,-1.801681803 
H,6.9181772715,0.8343778091,-1.8173681084 C,5.655771611,2.4388374203,-2.5614934093 $\mathrm{H}, 6.4486063165,2.8868867239,-3.1537114661$ C,4.3772183967,3.0057830632,-2.5746800827 H,4.1840124044,3.8876899797,-3.1783028484 C, $3.3379547837,2.4513538801,-1.8141193655$ C,3.6055938483,1.3212085183,-1.0370583814 C, $0.5398871674,1.5815091543,0.3100617516$ C,1.148119913,2.7972032781,0.6566434794 H, 2.2239559683,2.9089911323,0.5919335499 C, $0.4078426274,3.874375101,1.1653211404$ C, $1.0234808882,5.1131923261,1.5157290696$ H, 2.0905797918,5.2355877556,1.3480548621 C, $0.2710840054,6.1317696313,2.0552905536$ $\mathrm{H}, 0.7389341395,7.0761833057,2.3185838679$ C, $-1.1224579814,5.9551925147,2.2786393051$ H,-1.6991460194,6.7690076055,2.7097675762 C,-1.7459166691,4.7656782647,1.9589632952 $\mathrm{H},-2.8102856399,4.6319584237,2.1342162737$ C,-1.0019069313,3.6993844993,1.3884029536 C,-1.60208502,2.46487396,1.049679518 $\mathrm{H},-2.6493718563,2.3260210866,1.2916379725$ C, $-0.8844454738,1.4324320804,0.4698086816$ C,-1.5097227071,0.1540444145,0.1541908625 C,-3.9489994833,0.8811974897,-0.6240913629 C,-4.0029924172,2.2374581396,-0.9644724887 $\mathrm{H},-3.1336564038,2.8788065737,-0.8809843776$ C,-5.2042864486,2.7784617424,-1.4403747699 $\mathrm{H},-5.2437978287,3.8325270471,-1.7016645296$ C,-6.3489755639,1.9839402557,-1.5825550987 H,-7.27386964,2.4269343368,-1.9419540225 C,-6.3008647081,0.6150907439,-1.2878071073 H,-7.1737502769,-0.0156596531,-1.4342473883 C, $-5.1047271212,0.0710766435,-0.8303151672$ C,-4.7422147724,-1.3277131848,-0.5757000649 C, $-5.4287087018,-2.5219797245,-0.691200811$ $\mathrm{H},-6.4798767444,-2.5474875923,-0.964903698$ C,-4.7132438714,-3.7300758907,-0.497365258 H,-5.2273061181,-4.6754277914,-0.6455311912 C,-3.3677512946,-3.7451816304,-0.1675645937 H,-2.8645941908,-4.7007501586,-0.1217536755 C,-2.6467429115,-2.5231368911,0.0244669043 C, $-3.3743490278,-1.3405319354,-0.2174422239$ C, $-2.8485250092,-0.009935939,-0.1776541373$ C, $-0.6744597197,-1.0416479499,0.1978884757$ C, $-1.2442351271,-2.3664218661,0.2856972482$ C,- $-0.3642784222,-3.4800223533,0.5847377309$ C, $-0.8009460628,-4.6949794871,1.1630285363$ $\mathrm{H},-1.7804244403,-4.7515088027,1.6207809642$ C, $0.0267877738,-5.8065060503,1.1860341847$ H,- $0.3212330668,-6.7255278815,1.6485939036$ C, $1.3074866973,-5.7536540507,0.6016935557$ H, 1.9331646005,-6.6409656719,0.5763444003 C, $1.7888304001,-4.5496287932,0.1148231224$ H, $2.8026581346,-4.4786069226,-0.266886419$ C, $1.0011112721,-3.3831278907,0.1808044804$ C, $1.5629346664,-2.0907613594,-0.1536366375$ C, $0.7289722514,-0.8892412654,0.0483031496$ $\mathrm{H}, 2.21074088,-2.0891956585,-1.025903884$ H,2.3489636849,2.8978040957,-1.8331934829

\section{2-TS2-C3}

21

C, $1.3356407837,0.4291092382,-0.0145231274$ C, $2.710399936,0.364134179,-0.4229130626$ C, $3.9093034176,1.1788065243,-0.1698871642$ C, $4.1234209375,2.3508056554,0.5664326043$ H,3.3078541061,2.8866401093,1.037117752 C,5.4313686895,2.8315546778,0.7190493842
H,5.5973454817,3.7445714551,1.28322976 C,6.5171203278,2.1540307466,0.1577782927 H,7.5219338147,2.5488098351,0.2781508769 C,6.3251731581,0.9442166002,-0.5346662222 H,7.174068931,0.3900676242,-0.9250677351 C, $5.0351461391,0.4620686509,-0.679946216$ C, $4.5525683363,-0.8205328808,-1.2158551427$ C,5.1969596481,-1.9756731459,-1.6030703494 H,6.2807811835,-2.017367877,-1.6641144201 C,4.4313452226,-3.1575622725,-1.8643469885 H,4.9546605235,-4.0534537079,-2.1853882766 C,3.0620554434,-3.1784723765,-1.7377866875 H, 2.499339698,-4.0767474058,-1.9696927638 C, $2.3400658972,-1.985620428,-1.3646909391$ C,3.137892459,-0.8139260521,-1.092158492 C, $0.5437273286,1.6191348412,0.0960957037$ C, $1.0558866979,2.8995874326,-0.1501839514$ H,2.0191127778,3.0187097018,-0.6281654805 C, $0.3528744654,4.0552458394,0.2277929438$ C, $0.8879844086,5.3605790757,0.0216969394$ H, $1.8411473506,5.4628934504,-0.491046166$ C, $0.2094884078,6.4676405137,0.4820558302$ $\mathrm{H}, 0.6193609775,7.4621137905,0.3300212142$ C,-1.027392721,6.3177931462,1.1648794156 $\mathrm{H},-1.5476125876,7.2019455587,1.5233115091$ C,-1.5777474334,5.0666433288,1.3701147039 $\mathrm{H},-2.5316902566,4.9571473308,1.879504932$ C,-0.910989324,3.9076179052,0.8987458299 C,-1.4868466464,2.6155893893,0.9898740356 H,-2.4745196955,2.5180289414,1.427127392 C, $-0.8358209134,1.4976272242,0.5065391951$ C, $-1.5013009758,0.2079710229,0.3392455534$ C, $-3.9697535714,0.9281848779,-0.3934065204$ C, $-4.069643465,2.293895654,-0.6863091936$ H,-3.227162479,2.9633126289,-0.5781309078 C,-5.283977421,2.8159788512,-1.15074891 H,-5.350817004,3.8775135797,-1.3735363441 C, $-6.4042875492,1.9959893077,-1.3280449071$ H,-7.340781584,2.4235828014,-1.6757454277 C,-6.313681874,0.6202930823,-1.0818157074 H,-7.1651315589,-0.032478475,-1.2554642517 C, $-5.1042625368,0.096695146,-0.6364874483$ C,-4.7122598324,-1.3005904992,-0.4308144907 C, $-5.3683432263,-2.5035360681,-0.6193960657$ H,-6.411925288,-2.537334145,-0.9197812087 C,-4.6304341671,-3.7040543496,-0.4714580685 H,-5.1180409593,-4.6518381924,-0.6808231036 C,-3.2942262595,-3.7021785993,-0.1069738526 H,-2.7630107365,-4.644030136,-0.0909772374 C,-2.6114895962,-2.4747150124,0.1657194935 C, $-3.3527672618,-1.2951025546,-0.0462336709$ C, $-2.847991269,0.0463517431,0.0319179373$ C,-0.6726130309,-0.9840357423,0.4368309798 C, $-1.23161301,-2.3135370304,0.5217125075$ C,-0.3870161464,-3.4179328972,0.948053691 C, $-0.8959566006,-4.5872386111,1.5638338573$ H,-1.9516841046,-4.6734124533,1.7827537868 C, $-0.046222283,-5.6072365327,1.9667254341$ H,-0.4631290511,-6.4897291734,2.4434457557 C, $1.3463223838,-5.498147862,1.7901330036$ H,2.0014923825,-6.3057299074,2.102643192 C, $1.8754171845,-4.329877231,1.2679482512$ H,2.9502360669,-4.199441507,1.1758721016 C, $1.0257122152,-3.2801941689,0.8670683021$ C, $1.6015182979,-2.0151064173,0.482502848$ C, $0.7340310956,-0.8417014352,0.3310763559$ $\mathrm{H}, 2.5619843939,-1.8394722522,0.9557885925$ H,1.3599658506,-1.8735220614,-1.8233384564 


\section{2-TS2-C4}

21

C, $1.3860558667,0.324292528,0.1795378652$ C, $2.581711169,0.1777342882,-0.5580274929$ C,3.6608319683,1.1040535684,-0.9659214072 C,3.7265753969,2.4927975078,-1.1137583741 H,2.8650576774,3.1243505208,-0.9390535354 C,4.9283130392,3.0751861295,-1.5417657586 H,4.978857128,4.1529314768,-1.6663019343 C,6.053547998,2.2910623471,-1.81138863 H,6.9794802784,2.7647272966,-2.1250039113 C,5.9904866937,0.8907215386,-1.7075999744 H,6.8518590004,0.2760009902,-1.9532672098 C,4.794970886,0.3115867386,-1.3100266543 C,4.4009153434,-1.1037565027,-1.2514707198 C, $5.0927087711,-2.2874723489,-1.4113618699$ H,6.1639523115,-2.2874746965,-1.5914228515 C, $4.3923066647,-3.5291561108,-1.3211339141$ H,4.9478788699,-4.4514489097,-1.4625090901 C,3.0262240001,-3.5760840231,-1.1298913346 H, $2.4953037155,-4.5220361301,-1.1523562448$ C,2.2818661857,-2.3654799173,-0.9907720711 C,3.0143272806,-1.1372907429,-0.9517596211 C, $0.7110995637,1.5567174976,0.5618651082$ C, $1.3921077568,2.6647881439,1.0487800622$ H,2.4709958494,2.6405948623,1.1431718269 C, $0.7037160046,3.8026187201,1.5219863769$ C, $1.3945044149,4.9417287041,2.0251262608$ H, $2.4818687339,4.9361838609,2.0328864727$ C, $0.6906720584,6.034263584,2.4841216435$ $\mathrm{H}, 1.2205461022,6.905454364,2.8594296934$ C, $-0.7304608008,6.0312779453,2.4733030348$ $\mathrm{H},-1.2687524112,6.9004249949,2.841677982$ C, $-1.4278862642,4.93661884,2.0052471268$ $\mathrm{H},-2.5151769339,4.9298504297,2.0025745561$ C,- $-0.7320013737,3.7979190074,1.5156828522$ C,-1.4128343906,2.6457270077,1.0538963882 H,-2.4940186963,2.621871542,1.1329008655 C, $-0.7330778116,1.5417800387,0.5707284873$ C, $-1.4287084963,0.3090798145,0.1942156559$ C, $-3.6742844635,1.1175526165,-0.9725406916$ C,-3.6316071649,2.4790695454,-1.2886313383 H,-2.7751219723,3.0926279567,-1.0347456601 C, $-4.7143638479,3.058633256,-1.9627980951$ H,-4.6825985294,4.1173072734,-2.2060307139 C, $-5.8323147134,2.2960472349,-2.324544718$ H,-6.6650893251,2.7683262738,-2.8386829734 C, $-5.8757881663,0.9242787602,-2.0442381688$ H,-6.7284090458,0.323213942,-2.3491930524 C,-4.7978390701,0.3409383837,-1.384031942 C, $-4.5389968864,-1.065567031,-1.0483384109$ C, $-5.2381533415,-2.2402614427,-1.2586027749$ H,-6.2170642238,-2.2398075868,-1.7299952086 C,-4.6308673756,-3.4689670321,-0.8873203438 H,-5.150496592,-4.3977104387,-1.1048285396 C,-3.3857204302,-3.521440276,-0.2861107528 $\mathrm{H},-2.9441758608,-4.4893868889,-0.0882864244$ C,-2.6655565191,-2.3184317486,0.0046519703 C, $-3.2649094303,-1.1158513576,-0.4359180534$ C, $-2.6986430583,0.1977195627,-0.3420060392$ C,- $-0.7213059272,-0.9253970544,0.5116905503$ C, $-1.3690942663,-2.2068383965,0.6009075354$ C, $-0.6685184253,-3.297789613,1.27124342$ C,-1.333154294,-4.3740177319,1.8914857222 $\mathrm{H},-2.4143859028,-4.4132631311,1.9131411433$ C,-0.6175337008,-5.367850225,2.5574530975 $\mathrm{H},-1.1602922733,-6.1825776212,3.0281120223$ C, $0.7830925279,-5.3177655529,2.6497315434$ H, $1.3260443865,-6.1016169785,3.1686972707$ C, $1.4585935125,-4.2351271943,2.1089447781$
H, 2.5380363813,-4.1496791327,2.1993422309 C, $0.747986737,-3.2156163408,1.4384020549$ C, $1.4421652997,-2.0595435427,0.9786475198$ C, $0.6881081388,-0.8828967331,0.5688583761$ H,2.4361331507,-1.8949190622,1.3815741569 H,1.2501021224,-2.3725785982,-1.3309011499 $--$

\section{4-TS1-RC5}

21 C, $1.2580656521,0.0799491877,0.2114703419$ C, $2.0446809191,-0.0362559702,-0.8902582439$ C, $2.4626145882,1.0415181499,-1.8384576599$ C, $2.0568828587,2.371632308,-1.9984647757$ H,1.2914271022,2.8155999795,-1.3758753598 C,2.6340180205,3.1536466898,-3.008035454 H,2.3172234764,4.1869217259,-3.1251798508 C,3.6031205857,2.6196301576,-3.8661787088 H,4.0446995146,3.2422606229,-4.6399316761 C,3.9879023322,1.2794851777,-3.7450123896 H,4.7158682928,0.8481219759,-4.4273704528 C,3.4080693611,0.5004318707,-2.744639845 C,3.5620557031,-0.9299671996,-2.4656350988 C, $4.3380804083,-1.8854226284,-3.1195512609$ H,4.990970245,-1.5985114197,-3.9397542067 C,4.2508558938,-3.2209049196,-2.710091127 H,4.8462978142,-3.9803584157,-3.2101811154 C,3.3881389563,-3.5829428436,-1.6682460389 H,3.3114429003,-4.6246341354,-1.3675348167 C,2.6170351615,-2.6207534788,-1.0021898507 H,1.9569405083,-2.9421951284,-0.207784533 C, $2.7107427909,-1.2781279926,-1.3879323535$ C, $0.6276052587,1.3407061441,0.6827052646$ C, $1.3191614442,2.4803019232,1.0696878663$ H,2.4056266608,2.4706021816,1.0428389139 C, $0.6624261034,3.6990615371,1.4224041961$ C, $1.4077635973,4.8447139853,1.7820974029$ $\mathrm{H}, 2.4931695263,4.7941115288,1.7724278258$ C, $0.7565545634,6.0159656456,2.1483980293$ $\mathrm{H}, 1.3345469052,6.8954513965,2.4165568672$ C,- $-0.6490798039,6.0661703269,2.1836474548$ H,-1.148921189,6.9840024726,2.4795767005 C,-1.4048817689,4.9458558448,1.8465946159 H,-2.4905789115,4.9843412917,1.8767183358 C, $-0.7672703141,3.7616656203,1.4449060593$ C, $-1.5080052494,2.5673014605,1.1064252412$ $\mathrm{H},-2.4236661387,2.3984515569,1.6696019147$ C, $-0.7800358802,1.3871849952,0.6708842482$ C,-1.5996746829,0.4051977908,0.0266880172 C,-2.7189916096,1.0231248897,-0.5552604541 C,-2.6825372055,2.4745751492,-0.6050710358 H,-1.8358306581,2.9423121176,-1.1059005115 C, $-3.9284360146,3.1713678569,-0.6977215347$ H,-3.9410919476,4.2566877839,-0.7117918657 C, $-5.0842595984,2.4429508223,-0.8850676352$ H,-6.033842563,2.9564554265,-1.0013473548 C, $-5.0649506155,1.0256188688,-1.0092479594$ H,-6.0109555734,0.5201559017,-1.175957926 C,-3.8848865223,0.2916098748,-0.8746928698 C,-3.7452063873,-1.1609729092,-0.9850886771 C,-4.7479326862,-1.9581890998,-1.554407077 H,-5.6803826869,-1.5104264876,-1.8807721045 C,-4.5535750335,-3.3237159646,-1.7526823218 H,-5.3434415774,-3.913739351,-2.208523814 C,-3.3346536157,-3.9293476771,-1.409664972 H,-3.1696283096,-4.9823926836,-1.6162199272 C,-2.3330575508,-3.1730058975,-0.826357917 H, $-1.377545976,-3.6340332273,-0.6038643332$ C, $-2.5172878895,-1.7874673259,-0.5635014523$ C,-1.4881333053,-1.0217323214,0.0932796162 
C, $-0.4753292321,-1.6307816017,0.9431336765$ C, $-0.8331843816,-2.7131555238,1.7614177357$ $\mathrm{H},-1.8438142884,-3.1063402154,1.7254929711$ C, $0.0606352229,-3.2617223897,2.7031344796$ C, $-0.3121748451,-4.3559113675,3.5354639885$ $\mathrm{H},-1.3126262148,-4.7707517296,3.4416553972$ C, $0.5909515039,-4.8823137947,4.4358672309$ $\mathrm{H}, 0.3078969811,-5.7199101508,5.0670186429$ C, $1.8925958353,-4.3319196579,4.5445423049$ H,2.5934405626,-4.7561670389,5.2584509636 C. $2.2787427289,-3.2606889639,3.7557525441$ H,3.276949646,-2.840010237,3.8452689046 C, $1.3747776983,-2.7007146665,2.8226398581$ C, $1.7256093773,-1.5987820313,1.9915983612$ H, 2.7295888736,-1.1889702209,2.0654401051 C, $0.8493456521,-1.0717925726,1.0726554852$

\section{4-TS1-RC6}

21

C, $0.7868709201,-0.2339380231,-0.3687645714$ C, $1.8804252064,-0.2348349165,-1.2379146144$ C, $2.4817040265,-1.4773409835,-1.6288577485$ C, $2.0044611955,-2.8196985731,-1.4290863062$ $\mathrm{H}, 0.9812164004,-3.0683729919,-1.6980681936$ C,2.9648736567,-3.8809898436,-1.5402860075 H, 2.638310322,-4.9037560741,-1.3842076561 C,4.2518257509,-3.6044058742,-1.9445849589 H,4.9602385366,-4.416720767,-2.0769771506 C,4.6749081373,-2.2722369584,-2.2547667732 H,5.701790094,-2.0990106988,-2.5642211723 C,3.7901803951,-1.220142781,-2.1153155864 C,3.9237941535,0.239263546,-2.2645716921 C, $4.9408115686,1.0106075127,-2.8103964666$ H,5.8491059193,0.551285971,-3.1899875268 C,4.7614541551,2.4028542054,-2.8700023207 H,5.5432221917,3.0242556755,-3.297785493 C,3.5872018947,2.9983957421,-2.3938064892 H,3.4635287819,4.0752336629,-2.4644572357 C, $2.565751972,2.2250311524,-1.825094219$ H,1.6604326572,2.6977173655,-1.4607908158 C, $2.7400622847,0.842002486,-1.7559687215$ C, $0.2050568707,0.9247722058,0.3238844747$ C, $1.0746189756,1.737402044,1.042073543$ H,2.1480003151,1.6333198974,0.9166778784 C, $0.5942273999,2.6512532659,2.010412542$ C, $1.4719768486,3.4711897701,2.7707094744$ H, 2.5424096096,3.4057011255,2.5916680536 C, $0.9665858633,4.3341427798,3.7231859806$ $\mathrm{H}, 1.6398965774,4.9574348342,4.3052111123$ C, $-0.4302418456,4.409466582,3.9527288074$ $\mathrm{H},-0.8126775096,5.0919729185,4.7068658761$ C, $-1.3063378069,3.6235719246,3.2266867223$ H,-2.3780543121,3.6841128169,3.3988098907 C,-0.8172479742,2.7280698607,2.241452963 C, $-1.6862102897,1.9247477812,1.4547803649$ $\mathrm{H},-2.7574662858,2.0279614263,1.6035478113$ C,-1.2150986404,1.0443946173,0.4987333277 C, $-2.157485234,0.3211474685,-0.4012929318$ C,-3.1744995312,1.0862010866,-1.1085594614 C,-3.1714275549,2.5072075311,-1.1167593491 H,-2.3607261135,3.0465516853,-0.6423193132 C,-4.1754933853,3.22936278,-1.7361511336 $\mathrm{H},-4.1434471345,4.3155545979,-1.7307533294$ C, $-5.2306073401,2.5531001698,-2.3745283739$ $\mathrm{H},-6.0332072853,3.1124196301,-2.8477853541$ C,-5.2309629989,1.1698020075,-2.4282274861 H,-6.0362733202,0.6766196312,-2.9599902012 C,-4.1997186419,0.4036077372,-1.831284986 C,-4.1154736646,-1.041141055,-2.0009127934
C,-5.0601155699,-1.7662221769,-2.7674181469 H,-5.9268996172,-1.2615856401,-3.1774800272 C,-4.9010823502,-3.116443553,-3.0323427888 H,-5.6421311668,-3.6413005225,-3.6291090234 C,-3.7624636913,-3.7921594964,-2.5604722235 H,-3.6013506647,-4.83802085,-2.8076087724 C,-2.8345900161,-3.1149083906,-1.7886797922 $\mathrm{H},-1.9480400796,-3.6494156283,-1.4666577771$ C,-3.0070744361,-1.7466916611,-1.4440442532 C,-2.0838461184,-1.0482932195,-0.5631595233 C, $-1.0582342642,-1.858033832,0.1517121325$ C,-1.4222975987,-3.0378335565,0.814388266 H,-2.4775599192,-3.2746763906,0.9146804674 C,- $-0.4862438843,-3.9575874975,1.3559888336$ C, $-0.9091760838,-5.1271418587,2.0250802552$ H,-1.9715728247,-5.3278959305,2.1274859078 C, $0.0330478031,-6.0075825823,2.5468475093$ H, $-0.2930986412,-6.9083645113,3.0578315006$ C, $1.4066387353,-5.7340733785,2.4226964828$ H,2.1332590653,-6.4222184089,2.8445221166 C, $1.8454174829,-4.5801807452,1.7735126743$ H,2.906757125,-4.3690187553,1.6781689455 C, $0.9101847272,-3.6873273474,1.2281898247$ C, $1.3157551156,-2.4744670656,0.56989807$ H, $2.2856883871,-2.0753985805,0.8501724462$ C, $0.3070531733,-1.5309492561,0.0972521862$

\section{4-TS1-RC6-c1}

21

C, $1.3650974418,-0.0338833218,0.2876081401$ C, $2.3756962584,0.0284962212,-0.7275977583$ C, $2.4677060543,-0.5828823976,-2.0498012549$ C, $1.672204188,-1.5391404321,-2.6823059555$ H, $0.8474865208,-2.0118367986,-2.1570254151$ C, 1.9753786364,-1.8849550116,-4.0070838264 H, 1.3730169096,-2.631153749,-4.5166810069 C,3.0472844607,-1.2831235307,-4.6754780074 H,3.2654890537,-1.5654683537,-5.7014787124 C,3.8505400058,-0.3113581797,-4.0434957956 H,4.6745196721,0.1547747767,-4.5762451968 C,3.5586151663,0.0330564713,-2.7347109307 C,4.1839578254,0.9980684655,-1.8071746688 C.5.192200746,1.9298471118,-1.9138236784 H,5.7436729511,2.0623917467,-2.8400996435 C, $5.5026043391,2.7593304776,-0.7845438981$ H,6.3231992491,3.4647420528,-0.877113997 C,4.8274935546,2.661862532,0.4115482965 H,5.1257404098,3.2654265104,1.2619614401 C,3.7705862959,1.6934310535,0.5759421 H,3.6784502189,1.2456228426,1.5637585147 C,3.4703166727,0.9010509366,-0.5807785336 C, $1.0479132879,1.2129224272,0.9228617875$ C, $1.8875203071,2.4193310713,0.7095262022$ H,1.9415050079,2.7767185751,-0.3156484962 C, $1.8795660795,3.4593576266,1.7166976935$ C, 2.5829496656,4.6657246738,1.5440691929 H,3.1315524098,4.8396103614,0.6232839893 C,2.6046823079,5.6094023572,2.5622454043 H,3.1650005154,6.5305396653,2.4313151956 C, $1.9123678634,5.3788695205,3.7706948203$ H,1.947895045,6.1228939454,4.5609847341 C, $1.1600966077,4.2259190735,3.9376042531$ $\mathrm{H}, 0.5717153711,4.0694518632,4.8374274717$ C, $1.114127864,3.2616847634,2.9065922205$ C, $0.1299696359,2.2334442263,2.9020179667$ $\mathrm{H},-0.5872464452,2.2106154588,3.7184353738$ C, $-0.0280091288,1.3233075232,1.8647106254$ C, $-1.3362655282,0.6503159736,1.6503831682$ C,-2.5166594818,1.5125494453,1.6385562344 
C,-2.4306719821,2.9316180261,1.6739443046 $\mathrm{H},-1.4679854383,3.4223573503,1.6062878925$ C,-3.5592338645,3.7267965582,1.7630922532 H,-3.4569674734,4.8084041112,1.7872680052 C,-4.8316319882,3.131150274,1.8159095584 $\mathrm{H},-5.7225081697,3.7457100619,1.9135501851$ C,-4.9494447542,1.7569261374,1.7037797088 $\mathrm{H},-5.9429674737,1.3243971189,1.7056851944$ C,-3.8138003935,0.9198317386,1.5716742889 C,-3.9367067059,-0.5026725401,1.2852004313 C,-5.1945297294,-1.1172536297,1.0637328324 H,-6.107295606,-0.5452446195,1.1813252919 C,-5.2956118753,-2.437211789,0.6610792154 $\mathrm{H},-6.2746829323,-2.8772925363,0.4913848947$ C,-4.1300512027,-3.193149285,0.4388093112 $\mathrm{H},-4.1994275315,-4.2158994424,0.0780865237$ C,-2.8902469659,-2.6281721338,0.6743053212 H, $-2.0045077991,-3.2221632602,0.4851514971$ C,-2.7576356802,-1.2910439053,1.136463092 C,-1.4566691627,-0.7040096379, 1.4280193898 C, $-0.2744341287,-1.5914201811,1.5283855484$ C, $-0.3617666038,-2.7578515103,2.2637896516$ H,-1.2618272208,-2.9559392863,2.8379636911 C, $0.659320831,-3.7434734939,2.2721467229$ C, $0.5539661043,-4.9395120489,3.0244264977$ H,-0.3281146492,-5.1032451274,3.6381014863 C, $1.5597670005,-5.88678748,2.9682772067$ H, 1.4708709385,-6.8034245106,3.5451310548 C, $2.7127633759,-5.6793525499,2.1654397443$ H,3.4900909256,-6.4377309548,2.1385023481 C, $2.8479721859,-4.5218728637,1.4303172654$ H,3.7285235243,-4.3491524095,0.8168092554 C, $1.8233752144,-3.5311065488,1.4634663572$ C, $1.9352808604,-2.3216594641,0.7579972448$ H, 2.8658198127,-2.1338672682,0.2307488825 C, $0.9433336429,-1.325142225,0.8003973616$

\section{4-TS-RC6-C1}

21

C, $0.6837611397,1.1610665404,0.6542861755$ C, $-0.0305875738,1.2860553422,1.8454359007$ C, $-0.8356550311,0.3332495978,2.641618821$ C, $-0.8917464253,-1.0622764345,2.6750158514$ $\mathrm{H},-0.2484592831,-1.6682133529,2.0488074316$ C,-1.7901260892,-1.6815867836,3.5546696769 H,-1.833112374,-2.7663870036,3.5923825263 C, $-2.6292854061,-0.9243747745,4.3800619772$ H,-3.3273410289,-1.4256209159,5.044765307 C, $-2.5680591958,0.4780293655,4.3693471678$ $\mathrm{H},-3.2039751053,1.0667147506,5.0245373388$ C,-1.6627261257,1.0941081274,3.5150120182 C, $-1.3121058116,2.514289835,3.3612138316$ C,-1.8291216495,3.6796200715,3.8969682478 H,-2.689471097,3.6545362946,4.5597353184 C,-1.234802209,4.9319380756,3.5606067464 H,-1.6454296813,5.8382072574,3.9959497201 C, $-0.1181634496,5.0052507218,2.7502774016$ $\mathrm{H}, 0.3741234317,5.955963804,2.5722936436$ C, $0.4525380009,3.8149071032,2.2118344125$ H, 1.5139945508,3.8207829848,1.9845901591 C, $-0.2259381812,2.5840748444,2.4531685858$ C, $1.1575642663,2.3853201242,0.0274178653$ C, $0.3108800607,3.5840632891,-0.0071388801$ $\mathrm{H},-0.7629810268,3.4491183012,0.0700164965$ C, $0.7776055424,4.7844309746,-0.6210654612$ C, $-0.1122579833,5.869462007,-0.7816445308$ $\mathrm{H},-1.14276631,5.7596559737,-0.4547146317$ C, $0.3293118233,7.0659822002,-1.3304512264$ $\mathrm{H},-0.3580697582,7.8971685506,-1.4534359041$
C, $1.6695811771,7.1934301039,-1.7298563809$ $\mathrm{H}, 2.0167524783,8.1271788591,-2.1624224414$ C, 2.5643017401,6.1316858522,-1.5939136139 H,3.5806844377,6.2548022794,-1.9436665029 C, $2.1466934557,4.9075146059,-1.0432960344$ C, 2.9886069152,3.7452183399,-0.9239396068 C, $2.4223385052,2.4837070861,-0.517790678$ C,3.4786324887, 1.487481427,-0.4589504683 C,4.6751381126,2.1807387462,-0.7419222516 C,4.4091636822,3.5500889204,-1.0712297646 C, $5.5039314128,4.360405456,-1.4255935012$ H,5.392584389,5.4045955118,-1.691945059 C, $6.7761652902,3.7824661694,-1.443856053$ H,7.6268826532,4.397243451,-1.7226024643 C, 7.0052227902,2.4305729166,-1.1181979132 H,8.0243359094,2.0598032731,-1.155808196 C, $5.9405382602,1.5849232902,-0.7540174333$ C, $5.9956015006,0.1832765432,-0.3504757846$ C,7.2230351222,-0.4851979106,-0.1646867281 $\mathrm{H}, 8.1534275473,0.036342819,-0.3651251128$ C, $7.2701225135,-1.7930344562,0.2954141304$ H, $8.2300457558,-2.2819346435,0.4373400526$ C, $6.0793606828,-2.4713652674,0.6058672399$ H,6.1138593456,-3.4801432919,1.0077975414 C,4.8566521176,-1.8502654492,0.4100062631 $\mathrm{H}, 3.949674751,-2.3797348678,0.6790138261$ C, $4.7677172543,-0.5285308878,-0.0952459497$ C,3.4667275179,0.1158345575,-0.2843011342 C, $2.2035147216,-0.6333119583,-0.453274276$ C, $2.2371204627,-1.8277928803,-1.1664168457$ H,3.1870996276,-2.2040115974,-1.5305371601 C, $1.0747239345,-2.5618635616,-1.4983281333$ C, $1.1332130704,-3.7802914752,-2.2304049254$ H, $2.1028212332,-4.1633029288,-2.5393693921$ C, $-0.0255531589,-4.462746683,-2.5367152863$ H, $0.0236535249,-5.3952759887,-3.0924766585$ C,-1.2900890161,-3.9573080228,-2.1313089193 H,-2.1917188121,-4.5098941634,-2.3815324492 C,-1.3781674087,-2.7745639558,-1.4268976982 H,-2.3432605541,-2.3823760852,-1.1152774945 C, $-0.1990577754,-2.0531738979,-1.0934223098$ C, $-0.2370965745,-0.8374510279,-0.3727456664$ H,-1.2077549053,-0.4459475433, -0.0841223744 C, $0.908225764,-0.1279972355,-0.0464851889$

\section{4-TS-RC6-C2}

21

C, $-0.1340775788,1.3499677015,0.0437348213$ C, $-1.438933558,1.5545870292,0.6049756201$ C,-2.1259107134,0.9415555539,1.7493781111 C,-1.7827536937,-0.1313620372,2.573794076 H,-0.898264949,-0.7293344108,2.3770438898 C,-2.6149625193,-0.4328878257,3.6612000358 H,-2.3648932901,-1.2680363093,4.3091701866 C,-3.7637994963,0.3242813026,3.9174485067 H,-4.3957807889,0.0722247545,4.7643840588 C,-4.1109552497,1.4136636817,3.0968166779 $\mathrm{H},-4.9966865277,2.0055649781,3.3098611285$ C,-3.2922208536,1.7163670383,2.0192058452 C,-3.3613568654,2.7930834268,1.0145694358 C,-4.1793553554,3.8859138986,0.8362433461 H,-5.0196470717,4.074908222,1.498338555 C,-3.8977999093,4.8083383184,-0.2249452286 H,-4.5705106003,5.6488305039,-0.368313475 C,-2.827433751,4.6442855638,-1.0760939282 H,-2.6649265614,5.3376638488,-1.8944607674 C,-1.9501480237,3.5126234063,-0.9352251891 H,-1.4892442885,3.1356789406,-1.8454260988 C,-2.2492831391,2.6117923133,0.1420478765 
C $, 0.5541641423,2.5632683343,-0.3214426598$ C, $-0.0878980174,3.8873977164,-0.1244882414$ $\mathrm{H},-0.5281124755,4.0710541321,0.850283231$ C, $0.4853888437,5.0680027224,-0.7306123245$ C, $-0.133461334,6.3215719708,-0.5376145852$ $\mathrm{H},-1.0347460513,6.3777136309,0.0659423338$ C, $0.3721139402,7.4591389385,-1.1425849566$ $\mathrm{H},-0.1252965875,8.4147900237,-1.0060014307$ C, $1.525619295,7.3736372543,-1.9513720783$ H, $1.9028398972,8.2626541604,-2.4483682858$ C,2.1939967178,6.1702896826,-2.0986239614 H,3.0880640641,6.1316792824,-2.705941086 C, $1.7130062986,4.9970957017,-1.4704451915$ C, $2.4508430894,3.7759555959,-1.3872711134$ C, $1.8486902084,2.5823868244,-0.8505303768$ C, $2.8735594262,1.5617386794,-0.7432062979$ C,4.0868932796,2.178729644,-1.1510951889 C,3.8500357414,3.4937116045,-1.641242802 C,4.9412051185,4.2088730959,-2.1546995448 H,4.8485619347,5.211052785,-2.5537551945 C,6.2028071209,3.5951825425,-2.1476115822 H,7.0515360673,4.1411493222,-2.5492822254 C, $6.4149028429,2.312057968,-1.6240633722$ H,7.4216881065,1.9067421706,-1.6317096938 C,5.3412861444,1.5676515349,-1.0923128795 C, $5.3957902676,0.2783621241,-0.4143832373$ C,6.623597307,-0.3392185268,-0.0984593917 H,7.551645534,0.1202954236,-0.4232412094 C,6.6722695058,-1.5031130907,0.6539500716 H,7.6316838588,-1.9537871884,0.892713353 C,5.4829316272,-2.0739525194,1.1382940839 H,5.5183805902,-2.9552597829,1.7726607876 C, $4.2606538585,-1.5071150107,0.8161988727$ H,3.3557884345,-1.9485001622,1.2191433972 C, $4.1701735871,-0.3457430179,0.0067444583$ C,2.8669168491,0.2433534597,-0.3232231162 C, $1.6334262352,-0.553687258,-0.3958852476$ C, $1.762987159,-1.8847480918,-0.7809736996$ $\mathrm{H}, 2.7494941193,-2.2874556131,-0.9747439913$ C, $0.6708917569,-2.7542296599,-0.9970224304$ C, $0.841956649,-4.1088302089,-1.3863461904$ H, $1.8474668899,-4.501837683,-1.5126392438$ C, $-0.2617789636,-4.9114326279,-1.5953163746$ H,-0.1279215794,-5.9491854675,-1.888298705 C,-1.5787401369,-4.3980136657,-1.4366914974 H,-2.4302238881,-5.0502505428,-1.6092049461 C,-1.7749769393,-3.0844404899,-1.0727683593 $\mathrm{H},-2.7765970454,-2.6795607724,-0.9521685141$ C,-0.6528080123,-2.2366015367,-0.839651071 C, $-0.7960235686,-0.8885647176,-0.4825240542$ H, $-1.8079421422,-0.5009641472,-0.4434706046$ C, $0.2837402274,-0.0171369133,-0.2444274966$

\section{4-TS-RC6-T1}

21

C, $0.7352920764,1.5146258615,0.6818556845$

C, $-0.0121345423,1.598502272,1.8767281599$

C, $-0.5822120542,0.419912358,2.4584122604$

C, $-0.3372622327,-0.9616892851,2.1461255104$

H, $0.6868929541,-1.3183622126,2.058950955$

C, $-1.3096226557,-1.9188195516,2.589174624$

H,-1.1579625152,-2.9678971173,2.3573000807 C,-2.3592967088,-1.5169869342,3.384001388

H,-3.0639979395,-2.2542027045,3.7571347391

C, $-2.5173495263,-0.1527151659,3.7917764123$

$\mathrm{H},-3.3583149934,0.1215251467,4.4219790574$

C,-1.6222761243,0.7996269129,3.3503262526

C,-1.5191049633,2.2538272308,3.5430323126

C,-2.1855885079,3.0957011765,4.4223846232
H,-2.9762286723,2.7180545811,5.0646358028 C,-1.7975479645,4.4446217154,4.4799269656 $\mathrm{H},-2.3039124114,5.1194019807,5.1642007717$ C, $-0.7517079418,4.9199878605,3.6820482948$ H,- $0.4402595106,5.9574499603,3.7623471499$ C, $-0.0912227205,4.0760934849,2.7782953282$ $\mathrm{H}, 0.7275452885,4.4634367124,2.1839944813$ C,- $-0.4930420321,2.7408854685,2.6850053302$ C, $1.2490207013,2.6754220516,-0.0371374268$ C, $0.4652633105,3.8168828467,-0.226773316$ H, $-0.5617954509,3.8370857981,0.1213623314$ C, $0.9716895388,4.9540207076,-0.8963197961$ C, $0.1394924832,6.0936369007,-1.0882741065$ H, $-0.8949756732,6.043995925,-0.7574326936$ C, $0.6381275973,7.2402335221,-1.6704435474$ $\mathrm{H},-0.0022025764,8.1061920005,-1.8122181644$ C, 1.9909312906,7.2893822242,-2.0836023429 H, 2.3787328483,8.1948849197,-2.542517929 C,2.8273970981,6.199283062,-1.9142141791 H,3.8563740423,6.2639007843,-2.2448585071 C, $2.3506611838,5.0018283794,-1.3266154956$ C, 3.1411066204,3.8299794005,-1.1323041904 C, $2.551362259,2.6702960545,-0.5896512373$ C, $3.5523697213,1.625578515,-0.4863476403$ C,4.78793372,2.2135555679,-0.8871900569 C,4.5784614782,3.5419947342,-1.3417222472 C, 5.6769781698,4.2253976523,-1.8519065105 H,5.6098470088,5.2364740889,-2.2378657635 C, 6.9301337973,3.5659319116,-1.8816052476 H, 7.7877246948,4.103456233,-2.2771356962 C, 7.1047964888,2.2578743632,-1.4358700344 $\mathrm{H}, 8.0904671764,1.8072743936,-1.4932821803$ C,5.9996722019,1.5293441506,-0.9251453825 C,5.9839962789,0.164451068,-0.4105255451 C, $7.1754106411,-0.5701590656,-0.2248631261$ H, 8.1250852597,-0.1214357324,-0.4994645735 C, 7.1643663464,-1.8412198232,0.3287711651 $\mathrm{H}, 8.0977185642,-2.3798818628,0.4683121595$ C, $5.9481373042,-2.416644881,0.7362124055$ H,5.9374203963,-3.3942754901,1.2105110243 C,4.7597087117,-1.7287049988,0.5488310519 H,3.8372127279,-2.1764438327,0.9049932344 C,4.7285593599,-0.4438712359,-0.04830081 C, $3.4663921065,0.2808119025,-0.2223568735$ C, $2.1497717374,-0.4010944574,-0.2982130374$ C, $2.0933841944,-1.6627528819,-0.9150380385$ H,3.010898767,-2.0866012845,-1.3095818268 C, $0.9125569174,-2.4181607591,-1.0989036599$ C, $0.931102638,-3.665669721,-1.766441744$ H,1.8769374991,-4.0654765783,-2.1213076017 C,-0.2546820852,-4.3604142482,-1.9629064252 H,-0.2427773196,-5.3188586343,-2.4733778267 C,-1.478709987,-3.8250519192,-1.5131954215 H,-2.4000004088,-4.3735822317,-1.6857427189 C, $-1.5190910096,-2.5972701848,-0.8589320268$ H,-2.4624308763,-2.187064416,-0.509899372 C,-0.3265113071,-1.8850883137,-0.6394960792 C, $-0.3091203621,-0.6227961113,0.0372512555$ H,-1.249410752,-0.0822903844,0.0644612089 C, $0.9225727846,0.1693537095,0.132366077$

\section{4-TS-RC6-T2}

21

C,-1.1762659211,0.1717827215,-0.3201423735 C,-2.4941734556,0.3600140492,0.25296479 C, $-2.95861629,1.1493054581,1.3982726082$ C,-2.3088000083,2.0760976214,2.2160378228 H,-1.2830362886,2.3737524571,2.0221473988 C,-3.0148373599,2.6270141835,3.2951007911 
$\mathrm{H},-2.5245980848,3.3510659592,3.9393738959$ C,-4.3414165702,2.2605067647,3.5472964432 $\mathrm{H},-4.8711949755,2.7041475953,4.3855476182$ C,-4.9994813484,1.3166850823,2.7349271288 $\mathrm{H},-6.0244287904,1.0243175948,2.9458211186$ C,-4.3053588342,0.7637653899, 1.6708506206 C, $-4.6864893481,-0.263001145,0.683940491$ C,-5.765051687,-1.1009171747,0.540557994 H,-6.6184840551,-1.0448366005, 1.2101753456 C,-5.7454298384,-2.1002259831,-0.4940797711 H,-6.6246065654,-2.7259253804,-0.6186524363 C,-4.6706546354,-2.2730277777,-1.3324740537 $\mathrm{H},-4.7032049905,-3.016491846,-2.1215934618$ C,-3.5089860823,-1.4208156538,-1.2215787037 $\mathrm{H},-2.9843650926,-1.2021921929,-2.1492019642$ C,-3.568911308,-0.4181500607,-0.1926223419 C,- $-0.4234636697,1.3617485942,-0.6452563287$ C, $-1.1174170358,2.5856174382,-0.7603204367$ $\mathrm{H},-2.1993350182,2.6064948607,-0.6894308303$ C, $-0.463679695,3.8047596958,-0.9863758793$ C, $-1.221867511,5.0126488124,-1.0876443232$ $\mathrm{H},-2.3049742329,4.9532147084,-1.0164645397$ C,-0.5922168217,6.2215951527,-1.25838254 H,-1.1697992953,7.13872569,-1.3290928685 C, $0.825567416,6.2743741368,-1.3371848531$ H,1.315816336,7.2355718218,-1.4661821866 C, $1.5914915823,5.128234872,-1.2549562529$ H,2.6686835988,5.2074043273,-1.3207233746 C, $0.980544263,3.8586322733,-1.086904438$ C, $1.6814837527,2.6246061454,-1.0335433355$ C,0.9803522666, 1.4110434011,-0.8688980168 C, $1.9234561603,0.3192255959,-0.8753053878$ C,3.2211520659,0.8948544914,-0.9948735347 C,3.1234150564,2.3026783451,-1.1250602814 C,4.2997698061,3.013478525,-1.3278633282 H,4.3211245825,4.0899483262,-1.4500775742 C,5.5193557431,2.2963568572,-1.3903846204 H,6.4391871117,2.8537714351,-1.5455453692 C, $5.5870175262,0.9103968527,-1.2707918274$ $\mathrm{H}, 6.554697475,0.4238374315,-1.33457401$ C,4.4015712953,0.1593780624,-1.0733590861 C, $4.2728556587,-1.283843747,-0.9068826438$ C, $5.4080361567,-2.1156961293,-0.8040330723$ H,6.3971312625,-1.6804293375,-0.9022836708 C,5.2921485535,-3.4742349964,-0.5507143231 H,6.1843616727,-4.0891003921,-0.4695206065 C,4.0214736214,-4.0413446547,-0.3597804271 H,3.9232006257,-5.092987507,-0.1050710568 C, $2.8867903358,-3.2545239465,-0.4761860428$ H, $1.9236391008,-3.7118813235,-0.2785023641$ C, $2.9624891065,-1.8735064062,-0.7949909161$ C, $1.751607785,-1.0478281738,-0.915825432$ C, $0.4344757383,-1.6650791498,-1.1243746416$ C, $0.3912288132,-2.8243305561,-1.9183878095$ H, $1.2555069094,-3.0439164603,-2.5383439887$ C, $-0.6683296045,-3.7549949193,-1.9267090477$ C, $-0.5857766258,-4.9705445203,-2.651475301$ $\mathrm{H}, 0.2201030588,-5.1113704924,-3.3661741232$ C,-1.501957317,-5.9754513527,-2.4018449983 H,-1.4419392885,-6.9138192063,-2.9452488264 C,-2.5041294434,-5.8017610051,-1.416152048 H,-3.1985126722,-6.6116907634,-1.2122211089 C,-2.6166155148,-4.6084571448,-0.718929324 $\mathrm{H},-3.4048418711,-4.4696672174,0.0151713442$ C, $-1.7297566733,-3.5505919364,-0.9969202307$ C,-1.873081439,-2.2499712404,-0.3933814466 H,-2.3166887958,-2.2679763799, 0.5970176879 C, $-0.8260594319,-1.1988901464,-0.5949598293$

\section{4-TS-RC5-C1 \\ 21}

C, $0.6916903813,0.8759855621,0.0335771263$

C, $1.5761919769,1.6896306869,-0.6391241344$ C, $2.9029322049,1.2002359851,-0.8753383883$ C,3.4300394177,-0.0122744864,-0.389284647 C,4.7562363422,-0.3320883366,-0.8182243276 H,5.1967999985,-1.293234583,-0.5851419918 C, 5.477117009, $0.5597163849,-1.5973245222$ H, $6.4772017269,0.2851224097,-1.9204236052$ C, $4.9455862829,1.8103468826,-2.0041440252$ H,5.5473332394,2.4871604078,-2.6046152473 C,3.6448446844,2.1219011903,-1.6481384347 C,2.7579638239,3.2641632505,-1.9183344724 C, $2.9774425545,4.4274268827,-2.6518819046$ H,3.9425686708,4.6167193227,-3.1145822997 C, 1.9265056559,5.3439572916,-2.7911213944 $\mathrm{H}, 2.0808522781,6.2577859267,-3.3587764467$ C, $0.6764568345,5.0881827434,-2.2119976346$ H, $-0.131766311,5.8038345184,-2.3375874251$ C, $0.4511069227,3.9223630332,-1.4677517086$ H,- $0.5239888546,3.7445772681,-1.0287401655$ C, $1.4958705769,3.0096924906,-1.3053644837$ C, $-0.5925694076,1.3694060391,0.5972622319$ C, $-0.5296868056,2.5511607349,1.3119432117$ $\mathrm{H}, 0.4239447678,3.0609062309,1.4101265715$ C,-1.658105232,3.132935726,1.9443009698 C, $-1.5745456268,4.345408963,2.6736365738$ H,-0.6194117319,4.8599782404,2.7393518723 C,-2.6983381957,4.8616260912,3.2919675671 $\mathrm{H},-2.6298097367,5.7934358693,3.8468361411$ C,-3.9459663182,4.187527042,3.2154044493 $\mathrm{H},-4.8141115082,4.6108870285,3.7125909363$ C,-4.0554462006,3.0041432027,2.5176695436 H, $-5.0056400475,2.4806595369,2.4510690415$ C,-2.9170337956,2.4550204579, 1.8591871586 C, $-2.9866893315,1.2575778324,1.1251589183$ $\mathrm{H},-3.9297689503,0.7218637024,1.1199606482$ C, $-1.8717854909,0.7002857668,0.4777737828$ C, $-2.0378655139,-0.5688263443,-0.2060771537$ C, $-3.2629428483,-0.9224253548,-0.8853066354$ C,-4.1633413394,0.0810702992,-1.3379904551 H,-3.8796037224,1.1241365729,-1.2621423754 C,-5.3751011509,-0.2521019317,-1.9171163698 $\mathrm{H},-6.0408018893,0.5299263642,-2.2695840091$ C, $-5.7309040716,-1.602751368,-2.0597073791$ $\mathrm{H},-6.6854705801,-1.8693931807,-2.5045475207$ C,-4.8473775638,-2.6093732951,-1.6803295421 H,-5.1251318832,-3.6429262264,-1.8560400891 C,-3.5959127272,-2.3020724734,-1.1239835829 C, $-2.5932964682,-3.3321645203,-0.8625664491$ C,-2.8020192674,-4.7148495399,-0.8944921802 H,-3.7965667262,-5.1137166222,-1.0645092028 C,-1.7463722919,-5.63503624,-0.674746333 $\mathrm{H},-1.9702665676,-6.6972530788,-0.7022915084$ C,-0.4419937513,-5.2070354389,-0.5137495035 H, $0.3788673834,-5.9156616447,-0.4645061302$ C, $-0.1716058536,-3.8092704313,-0.5266678636$ $\mathrm{H}, 0.7648253687,-3.4973612588,-0.9858740262$ C, $-1.2869007415,-2.8911433787,-0.5512814434$ C, $-1.038531402,-1.5787581998,-0.0878660532$ C, $0.232961506,-1.5133307411,0.5753641428$ C,0.6428904194,-2.7891208566,1.1393721057 H,-0.1028979902,-3.3033579277,1.7397859187 C, $2.0124053349,-3.0080886413,1.4936929949$ C, $2.3860232634,-4.1368786524,2.2500938284$ H, 1.6312082545,-4.8779052838,2.4995379575 C,3.6986596032,-4.2916524844,2.6737608938 H,3.9892599006,-5.1627015685,3.2533969798 C,4.6363806363,-3.2892080637,2.3824527013 
H,5.6519380222,-3.372944294,2.7590409361 C,4.2800571149,-2.1662983042,1.6371054368 H,5.0102266393,-1.3786801839,1.5016416882 C, $2.9726330629,-2.0189309571,1.132344215$ C, $2.543090036,-0.8268484721,0.3973264261$ C, $1.1581599996,-0.4501445734,0.4097940958$

\section{4-TS-RC5-T1}

21

C, $0.8119138805,0.7203326095,0.0425528699$

C, $1.6822641496,1.5907843667,-0.590074341$

C, $3.0488180452,1.2016877658,-0.7289970928$ C,3.6093842162,0.041855801,-0.1758580048 C, $4.9875295757,-0.1720448549,-0.4759019662$ H,5.4820728328,-1.0932625345,-0.1952571472 C,5.7103384107,0.7687924383,-1.2003032032 $\mathrm{H}, 6.754780672,0.5656851158,-1.4215938672$ C,5.1324629922, 1.9731062657,-1.6723943458 H,5.7365761602,2.6941458387,-2.2162635626 C,3.7846473256,2.1778490589,-1.4423813569 C, $2.837576416,3.2453908196,-1.796035114$ C,3.0244836214,4.4099317265,-2.5370583745 H,4.0075313666,4.6653969291,-2.9241868952 C, $1.9218198541,5.2368513288,-2.7881214173$ H,2.0519242261,6.1494606801,-3.3639278051 C, $0.6500850165,4.8865532911,-2.316130183$ $\mathrm{H},-0.2008620622,5.5271736247,-2.5322875081$ C, $0.456828144,3.7188596218,-1.5658135932$ $\mathrm{H},-0.5420463779,3.4688052745,-1.2250657489$ C, $1.5526697212,2.8997995174,-1.2801371004$ C, $-0.4861678206,1.2001292907,0.5810998178$ C, $-0.587709535,2.4762687949,1.1288783136$ H, $0.3061470986,3.0867811866,1.2112980788$ C, $-1.8222665063,3.0687273657,1.5290275706$ C, $-1.863694685,4.3954817404,2.0177637346$ H, $-0.9430706453,4.9705710307,2.0656731583$ C,-3.0683401676,4.9507268216,2.425681149 H,-3.0972454688,5.9740702235,2.7884630073 C,-4.2548137463,4.1918344665,2.3818492305 $\mathrm{H},-5.1897835128,4.6349779549,2.7123646782$ C, $-4.2352951504,2.8776791475,1.9269496749$ $\mathrm{H},-5.1480037096,2.2888741468,1.8990916979$ C, $-3.0314512823,2.3089149471,1.4775305821$ C, $-2.9354845868,0.937756414,1.0418417328$ $\mathrm{H},-3.529008643,0.225638884,1.6121914788$ C,-1.6668840031,0.4350190124,0.5241582904 C, $-1.8004060384,-0.8036578119,-0.1585713289$ C, $-3.1093788098,-0.955762337,-0.6472361478$ C,-3.9246794318,0.2465445246,-0.6325122825 $\mathrm{H},-3.5369360161,1.1278604253,-1.1414276158$ C, $-5.3466593709,0.0867245095,-0.6385159481$ H,-5.9872857771,0.9625703987,-0.617252037 C,-5.8759444157,-1.1793953215,-0.7755781343 $\mathrm{H},-6.9526798218,-1.3134946476,-0.8179895642$ C, $-5.0482876224,-2.3247605182,-0.9313851727$ $\mathrm{H},-5.5319766196,-3.2897819065,-1.0422284983$ C,-3.6555367871,-2.2336586902,-0.8931260001 C,-2.7069719972,-3.3386804859,-1.037857828 C, $-3.1109217132,-4.5866461766,-1.5307299216$ H,-4.157527068,-4.7694425571,-1.7500803995 C, $-2.1827335828,-5.5921927399,-1.7953148158$ H,-2.5246512314,-6.544088563,-2.1913898875 C, $-0.812870434,-5.3586769691,-1.6104674031$ H, $-0.0854174776,-6.1168221834,-1.8840232881$ C, $-0.3851441132,-4.1429074158,-1.1030636417$ $\mathrm{H}, 0.6781203236,-3.9513791365,-1.0237092989$ C,-1.3106563707,-3.1224838239,-0.7465078261 C, $-0.8675778541,-1.8844502619,-0.1396009348$ C, $0.4181549387,-1.7785122629,0.5226200178$
C, $0.8989192595,-2.9183809512,1.1764730162$ $\mathrm{H}, 0.2296451412,-3.7527162111,1.3575850349$ C,2.2058030786,-2.9933999036,1.6879166615 C, $2.5961764601,-4.08340214,2.5130970669$ H,1.8655677169,-4.8579247865,2.7316443277 C, 3.8712034531,-4.1408043813,3.0357796777 H,4.1727236967,-4.9675715232,3.6721318282 C, $4.7738065875,-3.0905599224,2.7627125836$ H,5.7610343495,-3.100151074,3.2168179167 C,4.4207508459,-2.0296876537,1.9383297144 H,5.1274370884,-1.2210773231,1.8086888015 C, $3.1408116565,-1.9645247235,1.3417434351$ C, $2.6967996221,-0.8544080225,0.512134329$ C, $1.2982665126,-0.6119545453,0.3855211255$

4-R

01

C, $-1.1455358605,-0.3697907266,0.3853968747$ C, $-2.0811636421,-0.7278571497,-0.5361285463$ C, $-3.1219336684,0.1539586863,-1.1508211788$ C, $-3.3468077938,1.5364104077,-1.1017489722$ H,-2.6947976283,2.1989055047,-0.5489021393 C,-4.4339805737,2.0853694586,-1.7942162698 H,-4.5998964161,3.1590595863,-1.752047002 C,-5.3018630192,1.2751547454,-2.5382860179 H,-6.1434306368, 1.7192974565,-3.0641402489 C,-5.0747297516,-0.1023146437,-2.6198708063 H,-5.7260435284,-0.737007146,-3.2157689085 C,-3.9861118787,-0.6484029177,-1.9383053652 C,-3.4882838316,-2.0244957952,-1.9290706868 C,-3.9647152978,-3.1467509722,-2.6090579325 H,-4.8691614559,-3.084723042,-3.2092540351 C,-3.2478855454,-4.3441931494,-2.5226173551 H,-3.6017760783,-5.2284075447,-3.0470464936 C,-2.0610498168,-4.3986741859,-1.7790912258 H,-1.4906065829,-5.3233244414,-1.7382056475 C,-1.5905687754,-3.2761734719,-1.0861122801 $\mathrm{H},-0.6638761379,-3.3541450849,-0.5342606686$ C,-2.3173017307,-2.0797051497,-1.1319783321 C,-0.1366065483,-1.2975558127,0.9617399831 C, $-0.4154617989,-2.4155993781,1.7252209266$ H,-1.4413329314,-2.6437053528,2.0027549878 C, $0.6127603312,-3.3299182434,2.0911190631$ C, $0.3043612067,-4.4868621794,2.8610900599$ H,-0.72027939,-4.6255956012,3.1994703238 C, $1.2739005102,-5.417801977,3.1698781331$ H,1.0216165337,-6.29581858,3.7590636473 C, $2.6035785335,-5.2327219816,2.7140970071$ H,3.363925195,-5.9702917495,2.9589143423 C, $2.9410490048,-4.1241454567,1.9638413388$ H,3.9640096775,-4.0014868371,1.6281777426 C, $1.9706548414,-3.1372626989,1.6322779783$ C,2.2489810461,-1.9599627293,0.8805043992 C, $1.2152165059,-1.0339614074,0.6438457218$ C, $1.7310014904,0.0976491383,-0.1118259835$ C,3.0843438126,-0.2286340364,-0.4311220399 C,3.461064855,-1.4500998753,0.1942731441 C, $4.7838605375,-1.8566667715,0.0579542814$ H, $5.1642693692,-2.7641732255,0.5139567515$ C,5.6725007091,-1.0451076481,-0.6898402661 H,6.7042294156,-1.3705475742,-0.7978590694 C, $5.281556736,0.1572873142,-1.2755116951$ $\mathrm{H}, 6.0142560895,0.7425040835,-1.8229555665$ C,3.944499867,0.6088373195,-1.1388587572 C,3.3556687233,1.8409182831,-1.6561826932 C, $4.0606871475,2.6834580414,-2.5433355671$ H,5.0729028791,2.4193802583,-2.8349251694 C, 3.4814732362,3.8269089653,-3.0733517221 H,4.0453871764,4.4520458442,-3.7610280968 
C. $2.1562160654,4.157393664,-2.7400160437$ H, $1.6824278331,5.0308494675,-3.1806767423$ C, $1.4449690787,3.360252697,-1.8562921994$ H, $0.415482834,3.6149429773,-1.6277309878$ C,2.0149394666,2.2018868363,-1.271800355 C, $1.2331962349,1.3599968368,-0.3574525744$ C, $0.0915315633,1.874771541,0.4445939265$ C,0.1523244964,3.1691803291,0.9513836334 H,0.9819510211,3.8141500554,0.6792665214 C,-0.8024022629,3.6793450062,1.8648284904 C, $-0.7320945527,5.0102359268,2.3676024931$ $\mathrm{H}, 0.0766535861,5.656171066,2.0327656257$ C,-1.675211157,5.4717187587,3.2620489754 $\mathrm{H},-1.6159660702,6.4895504941,3.6394052129$ C,-2.729052797,4.6236211184,3.6968014266 H,-3.4653247467,5.0018123405,4.4015515419 C,-2.8194595863,3.3276962506,3.2313719265 H,-3.6241555851,2.6745087481,3.5616790086 C,-1.8604053605,2.8242680545,2.309429079 C,-1.9120726569,1.5027187471,1.8003904277 H,-2.7228052061,0.8513563237,2.1179956628 C,-0.99350831,1.0313642045,0.8825579827

\section{DDQ}

01

C, $-0.0661136564,-0.1250983946,0.0250911624$ C, $1.2898146115,-0.1251925179,0.0256031315$ C,2.0681965631,1.1596778965,0.0250451127 C, $1.2898223416,2.4349210263,0.0238749479$ C, $-0.0657747379,2.4350139245,0.023315269$ C, $-0.8443263821,1.1598815636,0.0238803888$ $\mathrm{Cl},-1.0066547926,3.8768045722,0.0219160738$ $\mathrm{Cl}, 2.2309037799,3.8765793885,0.0232656502$ C,-0.8376235117,-1.3267961094,0.0256151559 $\mathrm{N},-1.4671402521,-2.3030531528,0.0260457122$ C,2.0611603519,-1.3269967101,0.0266983023 N,2.6905376061,-2.3033433036,0.0275955656 $\mathrm{O},-2.0609298484,1.1378049701,0.023423516$ $\mathrm{O}, 3.2847745772,1.1374401267,0.0255549517$

\section{TfO-anion \\ $-11$ \\ S,-0.1364987038,0.0290548091,0.16480481 $\mathrm{O}, 1.3334235309,0.0633346467,-0.0322160292$ $\mathrm{O},-0.8333933973,1.3237140084,-0.0325462485$ C, $-0.7285125094,-0.9877629365,-1.30852795$ F,-2.0726925841,-1.1294677605,-1.3001122093 F, $-0.1882754625,-2.2266755092,-1.3000292547$ F,-0.3882023285,-0.4030086059,-2.4786899944 $\mathrm{O},-0.6032960618,-0.7727899442,1.3223784262$}

\section{DDQ-rad-ani}

$-12$

C, $-0.0882667606,-0.0854846819,0.0250338703$ C, $1.3119664521,-0.0855813517,0.0255779424$ C,2.0875367582,1.1449245911,0.0250558681 C, $1.2957562168,2.3899622851,0.023880851$ C, $-0.0717176741,2.390055289,0.023345047$ C, $-0.8636672239,1.1451283104,0.0239070924$ $\mathrm{Cl},-0.9941524392,3.8676025461,0.0219551594$ $\mathrm{Cl} 1,2.2183927954,3.8673824469,0.0232336656$ C, $-0.8229548477,-1.3040646817,0.0255807182$ $\mathrm{N},-1.4181597752,-2.306333025,0.0260351706$ C, $2.0464862495,-1.3042606157,0.0266982357$ $\mathrm{N}, 2.6415560522,-2.3066088217,0.0276165928$ $\mathrm{O},-2.1118134906,1.1476476968,0.0234449275$ O,3.3356843371,1.1472732923,0.0255597988

\section{DDQ-H-rad \\ 02}

C, $-0.0835570758,-0.084278198,0.0250289053$ C, $1.3086778059,-0.0665234474,0.0255803482$ C,2.0094125146,1.1706896293,0.0250163994 C, $1.2885626672,2.4015050596,0.0239042827$ C, $-0.0908697061,2.4075858372,0.0233355163$ C, $-0.8641049839,1.154474184,0.0238294699$ $\mathrm{Cl},-0.9931124042,3.8708590208,0.0219926342$ $\mathrm{Cl}, 2.2200485209,3.8717582612,0.0233085471$ C, $-0.8116991553,-1.3075971425,0.0255817076$ $\mathrm{N},-1.4079296884,-2.3065900786,0.0260332346$ C,2.0693644359,-1.276574388,0.0267168801 N,2.6823053302,-2.2638529933,0.027662421 O,-2.105202988,1.1422030851,0.0234102222 O,3.3352932152,1.0958665302,0.025616348 H,3.7664385017,1.9759367202,0.0251963933

\section{DDQ-H-ani}

$-11$

C, $-0.1015474425,-0.0605968093,0.0250114676$ C, $1.3237028791,-0.0440383845,0.0255786714$ C, $2.0302170629,1.1571908055,0.025046769$ C, $1.2905923702,2.3646986423,0.0239322238$ C,-0.0965408273,2.3692902717,0.0233607956 C, $-0.8954087928,1.1455452105,0.0238557931$ $\mathrm{Cl},-0.9845061897,3.8709800601,0.0219903484$ $\mathrm{Cl}, 2.2145502973,3.8622749689,0.0233038743$ C, $-0.8016209969,-1.292374522,0.0255800791$ N,-1.3839137311,-2.3051598876,0.0260363033 C, 2.0600299337,-1.265728302,0.0267130901 N,2.6538655051,-2.2679653806,0.0276292532 $\mathrm{O},-2.1546283998,1.1506183342,0.0233712443$ $\mathrm{O}, 3.3928536241,1.1023515939,0.0256571805$ H,3.7759816977,1.9983754789,0.0251462164

\section{DDQ-2H}

01

C, $-0.0957693383,-0.0360194994,0.0216312843$ C, $1.3195336787,-0.0360759608,0.0252734037$ C,2.03333714,1.1718465851,0.0253943059 C, $1.3112419577,2.3816479102,0.0218170939$ C, $-0.0872645102,2.3817040897,0.0182661922$ C,-0.8094668177,1.1719610426,0.0181056167 $\mathrm{Cl},-0.9841463663,3.8736379525,0.0138986918$ $\mathrm{Cl}, 2.2082528068,3.8735098666,0.0219632563$ C, $-0.8230195249,-1.2640187737,0.0214620016$ $\mathrm{N},-1.4142376832,-2.2654740853,0.0213281393$ C,2.0466767382,-1.264133598,0.0288948376 N,2.6378073884,-2.2656360946,0.0318748247 $\mathrm{O},-2.1549365132,1.094218827,0.0147975474$ $\mathrm{O}, 3.3788002673,1.0939957038,0.0289274021$ H,3.7983258858,1.9756532078,0.0289103665 H,-2.5743847091,1.9759090466,0.012254006 $---$

\section{4-P1-IN1}

11

C,0.8925407136,0.0410432862,-0.3659422856 C, $2.3362571657,0.135638878,-0.7514501633$ C, $3.4758034203,0.4420897199,0.0749802284$ C,3.5653758372,0.6993496914,1.4534739653 H,2.677164972,0.696559561,2.0766905964 C,4.8260613191,0.952454977,2.0083406932 H,4.9166881281,1.1546164069,3.0709835559 C,5.9647248414,0.9448933508,1.1991418868 
H,6.9360727236,1.1445164502,1.6432649857 C, 5.8881074956,0.6767176763,-0.1912567441 H,6.7889065894,0.6676567393,-0.7977045515 C,4.6503680763,0.4256560493,-0.7384536942 C,4.2287699142,0.091144919,-2.1166344091 C, $4.9359561624,-0.0605798893,-3.2871276173$ H,6.0126665643,0.0765198663,-3.3223080875 C, $4.2190330905,-0.4052671645,-4.4617417876$ $\mathrm{H}, 4.7644663742,-0.5290461371,-5.3931659732$ C, $2.8347858782,-0.5893320001,-4.4476854304$ $\mathrm{H}, 2.3143345286,-0.8547432374,-5.3625240168$ C, $2.1121628631,-0.4300380526,-3.2567905894$ $\mathrm{H}, 1.0360989156,-0.5732828424,-3.2482750014$ C, $2.8131514142,-0.0886316622,-2.0915420172$ C, $0.3008200129,1.3201099309,0.2540865209$ C, $0.9864801639,2.4774472581,0.5705521788$ $\mathrm{H}, 2.0662180605,2.5313156088,0.5169731093$ C, $0.3044439513,3.6766686807,0.9321673905$ C, $1.0440515651,4.8389742479,1.2859288373$ H,2.1300054938,4.777672348, 1.3105567972 C, $0.405135594,6.0224669515,1.593638239$ $\mathrm{H}, 0.9831422428,6.9016263149,1.8664624669$ C,-1.0093256128,6.0935657432,1.5535329097 H,-1.5088949678,7.0276144219,1.7979178511 C, $-1.7585613383,4.986477382,1.2080947017$ H,-2.8387213133,5.0619164296,1.1891070965 C,-1.1337058072,3.7480409074,0.8924177669 C, $-1.8316145066,2.563578901,0.522094749$ C,-1.113227311, 1.3717550802,0.2881267014 C, $-2.0410982344,0.3223663249,-0.1159535844$ C,-3.3215194744,0.9461144699,-0.2245097503 C,-3.2552797751,2.2943685566,0.2197488754 C,-4.4460970092,3.0124942475,0.2725981539 H,-4.4937227317,4.0404269398,0.6141312594 C,-5.6448414689,2.3711227303,-0.121242954 H,-6.5724468994,2.9363728499,-0.0806647848 C, $-5.6830386618,1.0447257521,-0.5471327819$ $\mathrm{H},-6.6360757886,0.6052760735,-0.8249510818$ C, $-4.4895322369,0.283073989,-0.5979464458$ C, $-4.3324264943,-1.1058586355,-1.0160114358$ C, $-5.3927257622,-1.8263879893,-1.6083802038$ H,-6.3571129945,-1.3439901472,-1.7367177979 C, $-5.2231525028,-3.127740718,-2.0563618639$ H,-6.0540143791,-3.6560713458,-2.5167017123 C,-3.9677755858,-3.749302391,-1.9369800964 H,-3.8179759828,-4.756690676,-2.3166399283 C,-2.9133901794,-3.0735758476,-1.3423734389 $\mathrm{H},-1.947352633,-3.562647698,-1.2764405504$ C,-3.0573539088,-1.75262394,-0.8484676137 C,-1.917591964,-1.0443790253,-0.2485520248 C, $-0.7775100494,-1.766364225,0.374340169$ C, $-1.06263824,-2.9212509771,1.0956691816$ H,-2.085236054,-3.281959598,1.1399989013 C,- $-0.0833795714,-3.6409876185,1.8210267868$ C, $-0.3886518373,-4.8290393253,2.5416614904$ H,-1.4125527709,-5.1955969903,2.5449245113 C, $0.6040351446,-5.5053089121,3.2218815161$ H, $0.3666415801,-6.4137382899,3.7695184028$ C, $1.9385997103,-5.0218605325,3.2130760473$ H, 2.7091433286,-5.5651854217,3.7539424888 C, $2.2596237313,-3.8670626144,2.5268745718$ H,3.280342949,-3.4911331526,2.5222476584 C, $1.2562957182,-3.1504490935,1.8207839018$ C, $1.5375792327,-1.9533652156,1.1107656747$ $\mathrm{H}, 2.5655737019,-1.6179534488,1.1241295901$ C, $0.5699995787,-1.2499042556,0.4196686391$ H,0.3425062998,-0.0687736406,-1.30829063

\section{4-P1-TS1-T1}

11

C, $0.9011288936,-0.1855666702,-0.5719267385$ C, $2.3154388306,0.0651161607,-1.0269837181$ C,3.2688465957,-0.9223820026,-1.0267208371 C,3.1120800867,-2.315645661,-0.621975928 H, $2.4228776103,-2.9373334848,-1.1902293398$ C, $4.3539618954,-2.9986317311,-0.2709625494$ H,4.3013028936,-4.0344244553,0.0490609956 C, $5.5652518584,-2.3922227174,-0.4484346105$ H,6.4725861688,-2.9543458152,-0.2414108082 C, $5.7035449684,-1.0383963308,-0.9484505464$ H,6.6949083544,-0.6032988352,-1.0395061172 C, $4.5801458284,-0.3290108703,-1.2444356261$ C,4.3399148505,1.0744257009,-1.6178154622 C, 5.1956485367,2.0814963346,-2.0425800954 H,6.2641381188,1.9051616148,-2.1361489375 C,4.6483067639,3.3387732109,-2.3584473764 H,5.3033329519,4.1380359623,-2.6948425182 C,3.2723474855,3.5707494961,-2.246524141 H,2.868876961,4.5472689112,-2.5007805688 C, $2.4043033315,2.5599818409,-1.8040791773$ $\mathrm{H}, 1.3403890514,2.752658018,-1.7073895179$ C, $2.9400323622,1.3109642507,-1.4889433805$ C, $0.2142708719,0.8508058254,0.3136123209$ C, $0.795351771,1.9155831083,0.9720000498$ $\mathrm{H}, 1.8730560684,2.0458764495,0.9782061156$ C, $-0.0083175263,2.9125153824,1.5961280848$ C, $0.6057909354,4.0183412762,2.2500009754$ H,1.6919884997,4.0555153258,2.2952700437 C, $-0.1510461558,5.0250778868,2.8098835734$ H,0.3333221448,5.8616031025,3.3068496554 C,-1.5666798633,4.9737175751,2.7355278567 $\mathrm{H},-2.1573799124,5.7718897946,3.1778256927$ C, $-2.1993066969,3.9200729685,2.1090238233$ H,-3.2818354185,3.8979933515,2.0680125105 C,-1.4529464535,2.8565901406,1.5265154211 C, $-2.0347075798,1.727132115,0.8869118508$ C,-1.1914508945,0.7204783207,0.3805899514 C, $-1.9945166708,-0.3568630574,-0.1766544754$ C, $-3.3502615186,0.0904948072,-0.118953692$ C,-3.4311510657,1.3342409778,0.5660978384 C,-4.6986985089,1.8583851454,0.7888706145 $\mathrm{H},-4.858280704,2.7935815284,1.3142973081$ C, $-5.8257272904,1.1332812598,0.325658415$ H,-6.8147771176, 1.5502490756,0.4976377009 C, $-5.7179849798,-0.095535168,-0.3205382854$ $\mathrm{H},-6.6208134714,-0.6110091413,-0.6330315626$ C, $-4.4386838934,-0.6661029905,-0.5457146945$ C, $-4.12617475,-1.9475009585,-1.1722703952$ C,-5.120029313,-2.7259098064,-1.8040735263 H,-6.1457324835,-2.3696328887,-1.815878621 C,-4.812958971,-3.9246641895,-2.4309032302 H,-5.597478351,-4.4989939812,-2.9165789328 C,-3.4832075355,-4.3807283988,-2.4575860153 H,-3.2313486404,-5.3013984735,-2.9773904594 C,-2.4870296545,-3.6467108679,-1.8321816032 $\mathrm{H},-1.4605823866,-3.9970095177,-1.888330765$ C,-2.7693325508,-2.4319216905,-1.1600058277 C,-1.6993019591,-1.6593036491,-0.5239925946 C, $-0.4367169571,-2.270320204,-0.0161180252$ C, $-0.495262585,-3.5485200391,0.5717846527$ $\mathrm{H},-1.4131408122,-4.1224862112,0.4917681524$ C, $0.5507101989,-4.1114334863,1.3339617325$ C, $0.41204326,-5.3863272085,1.9510632269$ H, $-0.4831532744,-5.9715860632,1.7580546121$ C, $1.4002692522,-5.8569759933,2.7922283535$ $\mathrm{H}, 1.2981209099,-6.8285215188,3.2671279034$ C,2.5452220429,-5.0668492269,3.0510772164 H,3.3097894546,-5.4396109386,3.7273920316 C,2.7036514842,-3.8234567898,2.4538449761 
H,3.5878960381,-3.2246588761,2.6545854702 C, $1.7219056887,-3.3341623053,1.5737776302$ C, $1.8664025453,-2.0683487319,0.8820148155$ $\mathrm{H}, 2.5117788969,-1.3448908584,1.3720189487$ C, $0.761417115,-1.5388852527,0.1210680201$ $\mathrm{H}, 0.29292751,-0.2299144913,-1.4892170695$

\section{4-P1-TS1-C1}

11

C, $-0.8859617254,-0.0661730095,-0.5592403889$

C,-2.2089669386,-0.6334184235,-0.9965733409 C,-3.4910835127,0.011810499,-1.3097456673

C,-3.8505872011,1.3516069372,-1.4695562173 $\mathrm{H},-3.1235135497,2.1456925503,-1.336579015$ C,-5.1785968438,1.6588579854,-1.804880263 $\mathrm{H},-5.4685359592,2.6981184862,-1.9348133282$ C,-6.1321347832,0.6474421793,-1.9678606964 $\mathrm{H},-7.1571719259,0.9079125836,-2.2180182882$ C,-5.7774196914,-0.705618975,-1.8170750274 $\mathrm{H},-6.5179601857,-1.4890008741,-1.9567006343$ C,-4.4626514661,-1.0162405555,-1.4983303384 C,-3.776199173,-2.3058925615,-1.3219768762 C,-4.2129229362,-3.5915839519,-1.2020537202 H,-5.2659100793,-3.8468105188,-1.2830844294 C,-3.2485198019,-4.633995093,-0.9259646659 $\mathrm{H},-3.6070349767,-5.6582434097,-0.8600814133$ C,-1.9094759586,-4.384963761,-0.7918794683 $\mathrm{H},-1.2119071968,-5.2026928318,-0.639333626$ C, $-1.3803848658,-3.0388094245,-0.9278945229$ $\mathrm{H},-0.4311827526,-2.9702193975,-1.4541727501$ C,-2.3694507981,-1.9957884646,-1.1409263479 C,-0.0634445326,-1.1014006554,0.2281512192 C, $-0.5651006709,-2.3256322819,0.7864719828$ H,-1.5395916242,-2.3139058086,1.2648689259 C, $0.3869192167,-3.2902146694,1.3215779547$ C, $-0.0845188808,-4.4274209143,2.0074759593$ H,-1.1527382907,-4.5336764423,2.1769577288 C,0.7929427602,-5.4084123337,2.4450797817 H,0.4123299102,-6.2818077226,2.9677497912 C, $2.1785330901,-5.2789185448,2.2110370905$ $\mathrm{H}, 2.860395228,-6.0508797611,2.5567224261$ C,2.6733570194,-4.165716628,1.5566671729 H,3.7416804628,-4.0632638672,1.4128801704 C, $1.8007094991,-3.1392778903,1.1091133273$ C, $2.2507575376,-1.9156848907,0.5271940583$ C, $1.2981732371,-0.8872605205,0.2283918116$ C, $1.9905009566,0.2797976517,-0.2925157215$ C,3.3524998773,-0.1057544307,-0.4158488328 C, $3.557181869,-1.4234137022,0.0929040966$ C,4.861974619,-1.9274628087,0.0793563307 H,5.106034621,-2.919745984,0.4413641155 C, $5.8922320338,-1.1069938708,-0.4162969763$ H,6.9069000934,-1.4953880104,-0.4288006329 C, 5.6656328497,0.1945897313,-0.8804555743 $\mathrm{H}, 6.5119057827,0.7747435964,-1.2351878627$ C,4.3617215011,0.7402185574,-0.8791194416 C, 3.9371773167,2.0677703786,-1.3163852134 C,4.8324758566,2.9630831323,-1.9400206924 H,5.8677636618,2.6695143418,-2.083534189 C,4.4153707491,4.2053818197,-2.3947227977 H,5.1254810339,4.8715403442,-2.8775765137 C, $3.070725047,4.5891509483,-2.2518682761$ $\mathrm{H}, 2.7313336853,5.5468393799,-2.6373746105$ C, $2.1714076833,3.7400349724,-1.6266316384$ $\mathrm{H}, 1.1322183346,4.0393673818,-1.543629998$ C, $2.5680413825,2.4763057418,-1.1233812356$ C, $1.593025226,1.5893351687,-0.4817778649$ C, $0.3271310728,2.0693738367,0.1391277101$ C,0.3464510059,3.2611099582,0.8538380151
H, $1.2367451693,3.8820602367,0.8446985034$ C, $-0.7391411716,3.6792142586,1.6636749429$ C, $-0.7160725178,4.9067345394,2.3834410812$ H, $0.1551585204,5.55186044,2.29513437$ C,-1.783360462,5.2712276952,3.178661766 $\mathrm{H},-1.7603767126,6.2115307636,3.7235093351$ C,-2.9167858464,4.4236358666,3.2929266422 H,-3.74954413,4.7243059084,3.9235639623 C,-2.9642933702,3.2244770472,2.6107204557 H,-3.8308819483,2.5724274014,2.6942605612 C, $-1.8800952068,2.8243220163,1.7830513067$ C, $-1.8873630815,1.6045086985,1.0589721706$ H,-2.7539309385,0.9570197584,1.1547109605 C, $-0.8516715038,1.2403911407,0.2262141189$ H,-0.3115305096,0.1107892767,-1.4834810179 $---$

\section{4-P1-TS1-T2}

11

C, $-0.8514951887,0.3036108493,0.6691301742$ C, $-2.3105582243,0.5996222466,0.8058313348$ C,-3.1932477723,0.1550920391,-0.1441129251 C, $-2.8443159973,-0.7324925569,-1.2460441873$ $\mathrm{H},-2.0437619105,-0.4215880859,-1.9144757971$ C,-3.9889942787,-1.3458549242,-1.9170335645 $\mathrm{H},-3.81053379,-1.9437696787,-2.8050004664$ C,-5.2641489476,-1.1370225265,-1.472020187 H,-6.0940690671,-1.5844690917,-2.0133528158 C, $-5.5699432426,-0.3329849358,-0.3057852629$ H,-6.595689307,-0.2798660514,0.0490652686 C,-4.5519696937,0.3039599417,0.3399714449 C,-4.4542812865,1.0451994516,1.6123479798 C, $-5.4138963268,1.5450243988,2.4812719156$ H,-6.4744252567,1.4350728371,2.2693878481 C,-4.9854156308,2.1999288914,3.6524557328 H,-5.7252590813,2.5947765049,4.3437159472 C, $-3.6232625862,2.347413501,3.939044063$ $\mathrm{H},-3.3175441213,2.8565785903,4.8491486012$ C,-2.6453459527,1.8484505389,3.0623791906 $\mathrm{H},-1.5877085003,1.9684546634,3.2829803636$ C,-3.0640568653,1.2012969688,1.9012486113 C, $-0.1576640706,1.3382824588,-0.2438980674$ C, $-0.805642158,2.405269173,-0.8369151853$ H,-1.8887356616,2.4797939448,-0.8189277714 C,-0.0777128349,3.4729976103,-1.4406096115 C,-0.7712160767,4.5535858926,-2.0541326434 $\mathrm{H},-1.8583059716,4.5236258412,-2.0820503401$ C, $-0.0881123888,5.6192735491,-2.6015766382$ $\mathrm{H},-0.6323768231,6.4353530893,-3.069785586$ C, $1.328376483,5.653849512,-2.5528445457$ H, 1.8609477074,6.4964373927,-2.9866161487 C, $2.0360431893,4.6266437551,-1.9622645777$ H,3.1187285737,4.6688946682,-1.941997825 C, $1.3653057055,3.5071380852,-1.3947765971$ C, $2.0205950222,2.3992981364,-0.7893778453$ C, $1.2498153291,1.3214029406,-0.3140511408$ C, 2.1279141963,0.2940489432,0.2080828002 C, 3.4504017317,0.8256996429,0.154752101 C, $3.4423195079,2.0951295811,-0.4840821531$ C,4.6709225734,2.7132337165,-0.6849835119 $\mathrm{H}, 4.7662384842,3.6788550255,-1.1693042589$ C, 5.8452546242,2.0470989672,-0.2508898924 H,6.8033085112,2.5371130007,-0.4050151426 C, 5.8242806482,0.7872765852,0.3431715457 $\mathrm{H}, 6.7613525768,0.321103405,0.6322824527$ C,4.5869142269,0.123962191,0.5469974545 C, $4.3604784612,-1.2054484035,1.1070125603$ C, $5.4112378612,-1.9588922844,1.6731584402$ H, $6.4120736453,-1.5382129072,1.6943775444$ C,5.1915810084,-3.2157898548,2.2173443024 
H,6.0187318154,-3.7707794818,2.6521200242 C,3.8951921528,-3.76004998,2.2221545743 H,3.7123495232,-4.7328809685,2.6708834483 C, $2.8429115533,-3.0489868636,1.6657817362$ H,1.8444111594,-3.4739534537,1.7036628405 C, 3.0345406558,-1.7711457885,1.0835435304 C, $1.9085113274,-1.0235497725,0.5180652558$ C,0.6496832949,-1.6983996389,0.0893338663 C, $0.8111416485,-2.8934709441,-0.6407317646$ $\mathrm{H}, 1.8219775921,-3.2302306375,-0.8533584232$ C, $-0.2513706701,-3.6744178459,-1.1356598939$ C, $-0.0169950348,-4.9007211717,-1.8154484742$ $\mathrm{H}, 1.0045933542,-5.1862549633,-2.0525354483$ C,-1.0807567516,-5.7209136983,-2.1364671775 H,-0.9116828682,-6.6620342951,-2.6516007405 C, $-2.3940269295,-5.3453850201,-1.7718953635$ $\mathrm{H},-3.2239587831,-6.0059023395,-2.0084521252$ C,-2.6405290596,-4.1438220758,-1.1182734582 H,-3.6542547198,-3.8684280505,-0.8442465284 C, $-1.5794847274,-3.2699583512,-0.8263404276$ C, $-1.7957646193,-1.9718522358,-0.1966644348$ $\mathrm{H},-2.5881269277,-2.0192096176,0.5468329122$ C, $-0.6481689335,-1.1585559179,0.1964930729$ $\mathrm{H},-0.3590081064,0.3912758823,1.6491210105$

\section{4-P1-TS1-C2}

11

C, $-0.8878783935,-0.3195501728,0.6291446598$ C, $-2.3844453538,-0.3336883471,0.7337791049$ C,-3.1709663759,0.4161866132,-0.1076812436 C,-2.6944911866,1.3485165223,-1.1182687805 $\mathrm{H},-1.9750361891,0.9976356028,-1.8533025194$ C,-3.6955930485,2.2743203445,-1.626706492 $\mathrm{H},-3.4181669135,2.9491497879,-2.4303742726$ C, $-4.9763544103,2.2652291825,-1.1448502355$ H,-5.707596898,2.9491172617,-1.5685593852 C,-5.4140583486,1.3655582081,-0.0986969396 H,-6.4257947914,1.4523230431,0.2882460503 C,- $-4.5292757261,0.457408073,0.4034639324$ C,-4.5691658313,-0.4753387927,1.5451154097 C, $-5.5986073858,-0.8985625587,2.3740320893$ $\mathrm{H},-6.6170567715,-0.5476475031,2.2279402983$ C,-5.2967376286,-1.7952746493,3.4175162795 H,-6.0921914154,-2.1353536425,4.0753764436 C,-3.9887918721,-2.2510290799,3.6212542291 H,-3.7808304562,-2.941683588,4.4341702001 C, $-2.9416042445,-1.8279677641,2.7859204399$ H,-1.9266188831,-2.1846846594,2.9421864327 C, $-3.236080368,-0.9436615045,1.7491384981$ C, $-0.4253106456,1.0919893946,0.211165246$ C,-1.3275943785,2.2150583039,0.0771022744 H,-2.1013391143,2.316140801,0.8316305869 C,-0.7929601418,3.5112265059,-0.3461214902 C, $-1.6623422224,4.6088607441,-0.4926740229$ $\mathrm{H},-2.7220685717,4.4713242842,-0.2991621431$ C,-1.1854734422,5.8484986005,-0.8961979047 H,-1.8750164094,6.679023292,-1.0209461971 C, $0.18840314,6.0311739895,-1.1518203826$ $\mathrm{H}, 0.5546156877,6.9970195117,-1.4885204128$ C, $1.0736646472,4.9845192161,-0.9653123242$ H, 2.1272441848,5.1341315129,-1.1667009621 C, $0.612470865,3.7116031266,-0.5420759684$ C, $1.475753998,2.6179119748,-0.2298516215$ C, $0.9223266619,1.3115398555,0.0084118064$ C, $2.0169793344,0.3839771374,0.2705145493$ C,3.1927030618,1.1794789879,0.3367588526 C, $2.91274482,2.5312877687,-0.0188371101$ C, 3.983683728,3.4340870587,-0.0501447645 H,3.8538750199,4.4821295406,-0.2935607867
C, 5.2696738296,2.957421701,0.2544155552 H,6.103368125,3.6537842216,0.2343029954 C,5.5202410967,1.6171484493,0.578200226 H,6.5410904587,1.3159669205,0.7925980626 C,4.4659245231,0.6792862379,0.6217088446 C, $4.5388559586,-0.7398073313,0.952621223$ C, $5.7287382272,-1.3312660235,1.4274538365$ $\mathrm{H}, 6.622827481,-0.7234818514,1.5251150884$ C,5.7747614359,-2.6677808061,1.7947923847 H,6.7023336884,-3.0964790274,2.1647758521 C, $4.6142593884,-3.4567302083,1.7120820093$ H,4.6344082129,-4.4945206043,2.0339500838 C, 3.4367800314,-2.9088191968,1.2276891179 $\mathrm{H}, 2.5445401775,-3.5243802294,1.1892869633$ C, 3.3614274024,-1.5555516911,0.8142772851 C, $2.0975378263,-0.9919654505,0.3253467959$ C, $1.0351309875,-1.8138586376,-0.3007926015$ C, $1.4330733119,-2.875264353,-1.1079685291$ H, $2.4850643045,-3.1318834433,-1.1769651182$ C, $0.5280475159,-3.6150667496,-1.9062222806$ C, $0.9467868884,-4.7036735777,-2.7213439069$ H, 1.9983303116,-4.9817198496,-2.7299193179 C, $0.0285360224,-5.3951460954,-3.4851052198$ H, $0.3518458605,-6.227654548,-4.1047008796$ C, $-1.3421418419,-5.0259245163,-3.4686532707$ $\mathrm{H},-2.0533463347,-5.5804404592,-4.0755167415$ C,-1.7733687312,-3.9696494957,-2.6902946623 H,-2.8229014992,-3.6842265819,-2.6754014099 C, $-0.8492674321,-3.2405851706,-1.8949092284$ C,-1.2443434915,-2.1467946152,-1.0795340983 H,-2.297216323,-1.8848887714,-1.077842322 C,-0.3577236134,-1.4379033338,-0.2947521999 $\mathrm{H},-0.4598765298,-0.5043378953,1.6263710385$

\section{4-P5-TS1 \\ 11}

C, $0.5939705592,0.9075087954,0.8310697788$ C, $1.9280228451,0.4622977207,0.854526572$ C,3.1245290694,1.0287177599,0.159583786 C,3.2539413834,1.6685926628,-1.0731214092 H,2.3951647913,1.8365496784,-1.7136055958 C,4.5288003743,2.0626432655,-1.4969444331 $\mathrm{H}, 4.642203503,2.563749265,-2.4540958787$ C,5.6548398486, $1.8099274827,-0.7022770851$ $\mathrm{H}, 6.6361161459,2.1277880051,-1.0446456991$ C,5.5342822712,1.1307181482,0.5171005571 H, $6.4124870729,0.9075096455,1.1166767038$ C,4.2679990598,0.7258727989,0.9343266564 C,3.8608407539,-0.0720987439,2.1020054944 C,4.6097092661,-0.615764853,3.1431973094 H,5.6833979283,-0.4579212213,3.1967807333 C,3.9474604011,-1.3716997932,4.1206653039 H,4.5163600301,-1.8067989332,4.9380115982 C, $2.5628123714,-1.5763294259,4.0562910134$ $\mathrm{H}, 2.0686404246,-2.1724671225,4.8183004453$ C, $1.8067853303,-1.0215546533,3.0143478481$ H, $0.7350198052,-1.1953700024,2.9628113197$ C, $2.4670347516,-0.2702844805,2.0475638294$ C, $1.0010196614,-0.7882566413,-0.2881655143$ C, $2.0366120201,-1.4237512644,-0.9333470846$ H,3.0433093996,-1.0278284424,-0.9408754392 C, $1.8411472413,-2.7017259854,-1.5232379909$ C, $2.9310343962,-3.3517096564,-2.1660213321$ H,3.8816758926,-2.8290376662,-2.2391345966 C, $2.7838921149,-4.6203331688,-2.6831306314$ H,3.6178316339,-5.1108421047,-3.1778307624 C, $1.5394671801,-5.2879657698,-2.5673946576$ H,1.426794226,-6.2883441654,-2.9769587947 C, $0.4666756659,-4.6841014684,-1.9436988666$ 
$\mathrm{H},-0.4697195193,-5.2207605754,-1.8826910238$ C, $0.5764097897,-3.3713251457,-1.4035006505$ C, $-0.4876654287,-2.6598775284,-0.7780943077$ C, $-0.3135087455,-1.3284681957,-0.3084564678$ C,-1.6305132555,-0.8033798996,0.1045604568 C,-2.5247010917,-1.9258870581,0.059501915 C, $-1.8842835851,-3.0541747836,-0.5096375419$ C,-2.6380786694,-4.2087081785,-0.7003823737 H,-2.2260092051,-5.1118547734,-1.1322351715 C,-3.9997528435,-4.2044198977,-0.3244034133 $\mathrm{H},-4.582907892,-5.1100519608,-0.4688707295$ C,-4.6197575163,-3.0756322271,0.2017855705 $\mathrm{H},-5.6755075793,-3.1179186679,0.4501870834$ C, $-3.8807366723,-1.8829057072,0.3906704457$ C, $-4.3878288795,-0.6162775792,0.8940687192$ C, $-5.6820380208,-0.4939657728,1.4448476373$ $\mathrm{H},-6.3266958866,-1.3658812117,1.4899106008$ C,-6.1379785638,0.7121604217,1.9535001136 H,-7.1354552226,0.7793578253,2.3798236652 C, $-5.2967206871,1.8385817635,1.9383219237$ H,-5.633405144,2.7785255875,2.3675514466 C,-4.0281566507,1.7499912812,1.3880032751 H,-3.3870240515,2.6238424394,1.4108737289 C,-3.5392639114,0.5384351544,0.8337555763 C, $-2.1755686797,0.4608450959,0.292067011$ C,-1.4962462514,1.7068474411,-0.1412562365 C, $-2.1939574984,2.6562091731,-0.8718296899$ $\mathrm{H},-3.2387207279,2.4903497841,-1.1161153063$ C, $-1.584399991,3.8523637488,-1.3271806779$ C,-2.3046144495,4.8263283057,-2.0715403169 $\mathrm{H},-3.3470658067,4.6376830421,-2.3158059489$ C,-1.6892847116,5.9948573603,-2.4707174692 $\mathrm{H},-2.2484770725,6.7348400421,-3.0371853225$ C, $-0.3284809516,6.2475909309,-2.1465285699$ H, $0.1352542735,7.1755903595,-2.4697768147$ C, $0.3986964576,5.3229535577,-1.4296701011$ $\mathrm{H}, 1.4415705686,5.5041518481,-1.1804033253$ C,-0.2063675743,4.1030426552,-1.0108778502 C, $0.516030199,3.1125716862,-0.317007706$ $\mathrm{H}, 1.5541700687,3.3011654234,-0.0696405309$ C, $-0.0923308327,1.9284698224,0.0875786211$ H, $-0.0276184668,0.4026893574,1.5657991282$

\section{4-P6-TS1}

11

C, $-0.8717698412,0.2875827182,0.2641984996$ C, $-2.0030221641,-0.5617844837,0.168517646$ C, $-3.3086303455,-0.2131885355,-0.4977981329$ C, $-3.5782738878,0.5798022613,-1.6109914361$ H,-2.7761850841,1.0270248185,-2.188775017 C,-4.9116797567,0.7649097262,-1.9997319845 H,-5.1356568058,1.3769412964,-2.869092707 C,-5.9536139866,0.1651412135,-1.280110434 $\mathrm{H},-6.982543164,0.3210614667,-1.5934219179$ C,-5.6844180349,-0.6329167502,-0.1617105625 H,-6.493536917,-1.0955230442,0.3969790584 C, $-4.3572907215,-0.8225969263,0.2238121336$ C, $-3.7937156277,-1.5885286697,1.3447354981$ C,-4.4141600119,-2.3690660653,2.3204249465 $\mathrm{H},-5.4941135223,-2.4881921756,2.3317297238$ C,-3.6161871109,-2.9969007989,3.2850548341 $\mathrm{H},-4.0832964925,-3.6100447268,4.0512656727$ C,-2.2227531021,-2.8453913814,3.2731501778 $\mathrm{H},-1.6179755846,-3.3423526782,4.0267485922$ C,-1.5985696025,-2.061156753,2.2933333069 H,- $0.5162757084,-1.9619061433,2.2789800148$ C,-2.3950264705,-1.435960343,1.3401631432 C, $-0.2629521481,1.3979731684,-0.4194165141$ C, $-0.8311606995,2.5832702073,-0.8811032504$
H,-1.9072251338,2.7157807099,-0.9071852695 C, $0.0039624541,3.6762956749,-1.2219913293$ C, $-0.578824363,4.8917405596,-1.6896943073$ H, $-1.6590750691,4.9372149314,-1.8041701163$ C, $0.202880821,5.9832794774,-1.9927240878$ H,-0.2537996168,6.9009515148,-2.352910136 C, $1.6114762701,5.9133052092,-1.83295714$ H, 2.2216420477,6.7787118236,-2.078161645 C, $2.2150238661,4.76116324,-1.3729042134$ H,3.2929912266,4.7251937302,-1.2684586019 C, $1.4464459249,3.6085732203,-1.0555529586$ C, $2.0075927884,2.3919513646,-0.5762213329$ C, $1.1537340745,1.3022164957,-0.3601971623$ C, $1.8984347191,0.1605693513,0.1324244614$ C, $3.2236324113,0.6454868555,0.3639664626$ C,3.3571469621,1.9789011506,-0.1165719614 C,4.6133260588,2.5667332021,-0.03470524 H,4.8076650522,3.57447722293,-0.3868431825 C,5.6771846451,1.81808629,0.5276995749 H,6.6569948119,2.283475052,0.5965863382 C,5.5188548376,0.5139892528,0.9916471197 H,6.3750594798,-0.0047369107,1.411889856 C, $4.252880155,-0.1196294591,0.9060039454$ C, 3.8866800384,-1.4620518553,1.3487952508 C,4.7800249982,-2.2738171473,2.0806091109 H,5.7730699117,-1.8994439264,2.30895614 C,4.4110724986,-3.5323306173,2.5326709004 H,5.1170804966,-4.1311846315,3.1019635853 C, 3.1196313331,-4.023666747,2.269687301 $\mathrm{H}, 2.8183627024,-4.9984377635,2.6438318765$ C, $2.2263863126,-3.2594690104,1.5365947657$ H, $1.2294876628,-3.6426823548,1.3439600066$ C, $2.5754196272,-1.9758491802,1.047769749$ C, $1.5955892678,-1.1841804812,0.2994852068$ C, $0.5260710142,-1.8649777715,-0.5093831892$ C, $1.0237318953,-2.8947212828,-1.3063934244$ H,2.041370377,-3.2330995419,-1.1414729673 C, $0.3266663005,-3.4648243271,-2.3943703139$ C, $0.8681218259,-4.522634361,-3.1758217202$ H, 1.8382581718,-4.9344408238,-2.9092632109 C, $0.1633233386,-5.0148570241,-4.2541177085$ $\mathrm{H}, 0.5763990682,-5.8260209624,-4.8478762941$ C,-1.1033976246,-4.4710747976,-4.6025967951 $\mathrm{H},-1.6402123405,-4.8729018086,-5.4577303375$ C,-1.6495209314,-3.442521918,-3.8657087509 $\mathrm{H},-2.6185331027,-3.0228636265,-4.1243342122$ C, $-0.9412540669,-2.9146833318,-2.74932473$ C, $-1.4728177271,-1.8753836239,-1.9544010941$ H,-2.4608908199,-1.5221661778,-2.2123206012 C, $-0.8238593952,-1.4131451329,-0.8181935007$ H,- $0.2353809763,-0.0425958902,1.079668412$ $---$

\section{4-P2-IN1}

11

C,-0.4497695771,1.0047343688,-0.2653249394 C,-1.3928296464,1.8115421279,-1.1619948681 C, $-2.5579680536,2.586185636,-0.5383844449$ C,-2.5663829388,3.4406429928,0.5591805879 H,-1.6707850197,3.6019103344,1.1533098294 C,-3.7662876928,4.0791923503,0.905035797 H,-3.791444823,4.7448371292,1.7638517125 C,-4.932237199,3.8622833423,0.1572993498 $\mathrm{H},-5.8535791122,4.3652906381,0.43966418$ C, $-4.9254985496,2.9962534908,-0.9419987681$ $\mathrm{H},-5.8333465801,2.8203510592,-1.5134722419$ C,-3.7338892453,2.3512077348,-1.284280776 C, $-3.4490591058,1.3567632661,-2.3242672791$ C,-4.2816993192,0.7689819563,-3.2815809846 $\mathrm{H},-5.3271321512,1.05694268,-3.3578067146$ 
C, $-3.7452044136,-0.2015389376,-4.1346879869$ H,-4.3803919598,-0.6716376783,-4.8811338798 C,-2.3970554211,-0.5774512214,-4.0360212236 H,-1.9984635464,-1.3362534437,-4.7044148562 C, $-1.5581487604,0.0167607039,-3.083083657$ $\mathrm{H},-0.5145029702,-0.2782138765,-3.0044334935$ C, $-2.0950450491,0.9779525618,-2.2334464016$ C, $-0.9561090006,-0.1949997365,0.3358500836$ C, $-2.3437812354,-0.3200982137,0.5788963635$ $\mathrm{H},-3.0059500255,0.5106465317,0.3829953302$ C, $-2.9143985225,-1.4677465356,1.1396164477$ C,-4.3190250409,-1.5022175482,1.4080781505 H,-4.9092329638,-0.6143342184, 1.1949936667 C,-4.905435747,-2.6338981251,1.9192811424 H,-5.9723478828,-2.6600588314,2.1212789494 C,-4.1085344579,-3.7797244417,2.1870349456 $\mathrm{H},-4.578293075,-4.6711783346,2.5943795301$ C,-2.7494446882,-3.7825530403,1.9429043517 H,-2.1761599677,-4.6722488128,2.1666806613 C,-2.1031016791,-2.6328654462,1.418078273 C, $-0.7136458919,-2.5403698678,1.1371496954$ C, $-0.1504104545,-1.3255746009,0.6818333211$ C, $1.2528596253,-1.5384880266,0.4092584788$ C, $1.5181058085,-2.9180184128,0.6575915265$ C, $0.3589947378,-3.5597137808,1.1578982838$ C,0.4728427581,-4.897116778,1.5244713396 H, $-0.3552592654,-5.4684520426,1.9272381244$ C, $1.7270030079,-5.5356764099,1.3763375927$ H, 1.8127089333,-6.5801774651,1.6643168054 C, $2.8523537924,-4.8799603132,0.8809101121$ H,3.7861949788,-5.4263899246,0.7928235914 C, $2.7664884805,-3.5172119772,0.5049373053$ C, $3.8143731173,-2.6795555221,-0.0679521176$ C, 5.0633433081,-3.2179610556,-0.4445933526 H,5.2660640578,-4.2683223425,-0.261838125 C, $6.0266725522,-2.443215749,-1.0725947782$ H,6.9769943755,-2.8859919091,-1.3588775384 C,5.7555559622,-1.0960839639,-1.3674307323 H,6.4859938349,-0.4950153772,-1.9022740766 C,4.5482586245,-0.5329132053,-0.9862148271 $\mathrm{H}, 4.3492170126,0.5006768961,-1.2463218638$ C,3.5576797882,-1.2818928521,-0.3009335453 C, $2.2757008543,-0.6748551316,0.0845344519$ C, $2.1081870026,0.7762914263,0.2584588615$ C,3.1876607381,1.4883262093,0.7605621998 $\mathrm{H}, 4.0741740237,0.9473292143,1.0729684293$ C,3.206258612,2.8950510573,0.8956167863 C,4.3138038657,3.5960598013,1.4378793921 H,5.1746410356,3.0353469809, 1.7927413714 C,4.2934815025,4.975284057,1.5042850674 H, 5.1447965458,5.5079866885, 1.9196428999 C,3.1723179957,5.7144539101,1.034734226 H,3.1826229321,6.798905912,1.0979536798 C,2.0805990792,5.0609597269,0.5099732481 H, 1.2143215399,5.6133255628,0.1544196648 C, $2.0735437681,3.6360745622,0.4293587306$ C,0.9697405348,2.9298808556,-0.0658032054 H, $0.113451415,3.5279020268,-0.3471394025$ C, $0.8865574327,1.5160062327,-0.0790334915$ H,-0.7841186011,2.5320921253,-1.7142789658

\section{4-P2-TS1-T2}

11

C, $0.9066897499,0.0152236884,-0.4397752229$ C, $2.3345701319,0.0816976889,-0.9600324325$ C,3.2233453924,-0.5728693481,0.047007915 C, $2.8294440416,-1.7142718347,0.8093068609$ H,2.0754460363,-1.5512501077,1.5838770351 C,3.9346847312,-2.4898608348,1.3499482038
H,3.7131666693,-3.365450135,1.9521195541 C,5.2370296145,-2.0557876647,1.2013009567 H,6.0481569295,-2.6309675367,1.6383422916 C, 5.5488712483,-0.8458877413,0.5267345482 H,6.5882604978,-0.546585844,0.4193681276 C,4.524035892,-0.0857042084,-0.048768577 C,4.4928194763,1.1028663706,-0.9187858208 C, $5.5058308448,1.9924468374,-1.2849446641$ H,6.5179781238,1.8676850496,-0.9090762333 C,5.1861131969,3.0537273378,-2.1383206166 H,5.9572467592,3.7634207653,-2.4259701276 C,3.8805651947,3.2114868488,-2.6277385602 H,3.6518000191,4.0433669722,-3.2887137686 C,2.8657840477,2.3113061762,-2.2756009729 H, 1.8563969332,2.4378534361,-2.656745892 C,3.1737378984,1.2687358725,-1.409315713 C,0.2837734428,1.2420063853,0.0769364983 C,1.0668498884,2.330479459,0.464424055 H,2.1467815514,2.2596488125,0.4612268647 C,0.5102917962,3.5607165236,0.8892689648 C, $1.3623498867,4.6364599776,1.2731989816$ H,2.4383054688,4.4771567139,1.2592337574 C, $0.8384425284,5.8562817525,1.6421636451$ H,1.4966001625,6.67157063,1.9305668369 C, $-0.5663820491,6.0512992433,1.6410912186$ H,-0.9743741186,7.0162576551,1.9311407345 C,-1.4213865046,5.0295734409,1.2777814406 H,-2.4895892389,5.2047181616,1.2899704003 C,-0.9175350813,3.7553462301,0.8985297445 C,-1.721688211,2.6362826944,0.5446449094 C,-1.1201318322,1.4021241219,0.2180758962 C,-2.1591750481,0.4265539448,-0.0526999649 C,-3.4023832467,1.1227721032,0.0276420828 C,-3.1885400083,2.4662101831,0.4305114438 C, $-4.3113065478,3.2588228286,0.6430413523$ H,-4.2473302334,4.2928841547,0.9618833231 C, $-5.5926006231,2.6871154108,0.4504898409$ H,-6.4673668563,3.3109098928,0.6157373508 C,-5.7749428774,1.3588471705,0.073125061 H,-6.7845662624,0.9776860731,-0.0441542384 C,-4.6490034462,0.5243503029,-0.1403287892 C,-4.6360920597,-0.8788180964,-0.5374315913 C, $-5.8220030487,-1.5452089246,-0.9184611123$ H,-6.7669218356,-1.0126173921,-0.8758498343 C,-5.8051348836,-2.8529176698,-1.3765986265 H,-6.7319472401,-3.3382837462,-1.6711756776 C,-4.5804455866,-3.5336291025,-1.4885864659 H,-4.5496996115,-4.5431173045,-1.8902738311 C,-3.4041459422,-2.915460242,-1.0958196216 H,-2.4714696683,-3.4548169685,-1.2127891203 C,-3.3852961904,-1.5934003836,-0.5808705308 C, $-2.123382215,-0.9388930003,-0.1877796924$ C, $-0.9010550142,-1.7215709851,0.1213496973$ C,-1.0614055037,-2.9318313934,0.7494932133 H,-2.042532168,-3.1992587166,1.1280964278 C,-0.0520559054,-3.9540986167,0.8032504324 C, $-0.3202026499,-5.2292264156,1.3465900672$ H,-1.2638718828,-5.3993418404,1.8592666411 C,0.5988950518,-6.2617157213,1.2008236776 H,0.3857611438,-7.2402564405, 1.6230005547 C, $1.7965815011,-6.0501565826,0.4920957826$ H, 2.5061894417,-6.8633832849,0.3672635845 C,2.0763682399,-4.7941078942,-0.0369537222 H,3.014272619,-4.6154863457,-0.5582004635 C, $1.1711917572,-3.7337117729,0.1315459579$ C, $1.5040140773,-2.3822358552,-0.335162663$ H,2.1088111168,-2.4042795101,-1.2406821274 C, $0.4462898893,-1.2967235435,-0.2920324515$ H,2.373088599,-0.5850259054,-1.8398508062 


\section{4-P2-TS1-C1}

11

C,-0.1359729121,-1.5048809796,0.2182129729

C, $-0.0989646832,-2.7117367392,-0.7654668011$ C, $1.2333096349,-2.6723071646,-1.4873138179$ C, $2.5325672143,-2.7500273122,-0.8821565096$ $\mathrm{H}, 2.7717455565,-3.6864357455,-0.3725182226$ C,3.6457912074,-2.2802135349,-1.6829310435 $\mathrm{H}, 4.6449890008,-2.3524567821,-1.2647113153$ C,3.4587209007,-1.7795404744,-2.9551046944 H,4.3062343084,-1.4239113352,-3.5331643376 C, $2.1689430786,-1.7461648266,-3.5229482939$ H,2.0267159695,-1.3396907446,-4.5208318744 C, $1.0626896471,-2.1898445416,-2.7820914439$ C, $-0.3711728524,-2.1610575832,-3.0913177905$ C,-1.0278033205,-1.8872247956,-4.2921133428 H, $-0.46690355,-1.6063746729,-5.1796654832$ C,-2.4209352902,-2.001112728,-4.3305815585 $\mathrm{H},-2.9550094554,-1.7961599128,-5.2546249032$ C,-3.1304409018,-2.3954534053,-3.188434726 H,-4.2112338324,-2.500344275,-3.235014965 C,-2.4672845607,-2.6587132518,-1.9811279168 $\mathrm{H},-3.0391831621,-2.9619202497,-1.1124877514$ C, $-1.0823294818,-2.5225833052,-1.9283765932$ C, $1.071262204,-0.9958499439,0.6471218114$ C, $2.2496167083,-1.9034632669,0.7485425608$ H,1.9666806488,-2.8215465308,1.2574770891 C,3.5069526797,-1.3545346132,1.2961041059 C, $4.5394248248,-2.2408861906,1.6457009277$ H,4.3820732694,-3.3100195114,1.5180958838 C, $5.754228689,-1.7694539636,2.1321920994$ H,6.5440162783,-2.4657290962,2.4000531806 C, $5.946468683,-0.3867826723,2.2787189338$ H,6.8904644761,-0.0068239459,2.6606849892 C,4.9296045566,0.5057050985, 1.9531741166 H,5.0913882223, 1.5651077298,2.1066259203 C,3.6896862857,0.0481144226, 1.4601534493 C, $2.5683213979,0.9203674108,1.1712575097$ C, $1.2987561909,0.3920875104,0.9206244405$ C, $0.3833293498,1.474826485,0.5885779768$ C, $1.161305618,2.6671338166,0.5956663174$ C, $2.4934729035,2.3823494544,1.0011446401$ C,3.3714710369,3.4586810628,1.1144586434 H,4.4050596092,3.3417654602,1.420080032 C, $2.8926187988,4.7573967736,0.823746462$ H,3.5806804118,5.5942513143,0.9131355272 C, $1.5791737369,5.0073488359,0.4263001547$ $\mathrm{H}, 1.2797411313,6.0281627386,0.2100847663$ C, $0.6613842705,3.9351238255,0.3050444605$ C, $-0.7234038794,3.9858064229,-0.1524921195$ C,-1.2957397662,5.1799640241,-0.6427890142 H,-0.7131101462,6.0958080946,-0.6254506018 C, $-2.575716873,5.205714133,-1.1754308388$ H,-2.9872512383,6.137799949,-1.5539133959 C, $-3.3225715954,4.0175858622,-1.254789359$ H,-4.3071725897,4.019035915,-1.7150258317 C,-2.7998684059,2.8362579829,-0.7530954095 $\mathrm{H},-3.3817857148,1.9259363346,-0.844562077$ C, $-1.5136105963,2.7808483047,-0.1581580558$ C, $-0.9750434861,1.515997431,0.3625851215$ C, $-1.8391603206,0.3858660608,0.7689044321$ C, $-3.0650014112,0.6781390457,1.3594781346$ H,-3.3530838734,1.7150000393,1.4963422998 C, $-3.9576572603,-0.3104863536,1.8299451824$ C,-5.2093673719,0.0158909345,2.4244736887 H,-5.4871481875,1.0627431662,2.5242210437 C,-6.0553023705,-0.9824374048,2.8591599559 $\mathrm{H},-7.0119978237,-0.7288443382,3.3083583517$ C,-5.6859231921,-2.3488416936,2.7239137397
$\mathrm{H},-6.3651796814,-3.1232708383,3.0709362522$ C, $-4.4758737754,-2.693951283,2.1598660956$ $\mathrm{H},-4.1887790769,-3.7381707177,2.0581752858$ C, $-3.5840359774,-1.6841050258,1.6993217435$ C,-2.3280408855,-1.9838591788,1.1278186588 H,-2.039100005,-3.0298570376,1.0740048497 C,-1.4442348553,-1.0068039672,0.6767011558 $\mathrm{H},-0.2527421378,-3.6551207062,-0.2217798747$ $---$

\section{4-P2-TS1-C2}

11 C, $1.248759741,-0.2857307826,-0.3065108017$ C, $2.3799089652,-0.2304230484,0.7075548666$ C, $2.8261016134,0.8923065028,1.6302357678$ C. $2.0840600712,1.7222282771,2.4627465845$ $\mathrm{H}, 0.9980948421,1.7023622701,2.4438200856$ C, $2.7672812214,2.6033579674,3.3121040482$ H,2.2015480066,3.2724680528,3.9551536138 C, $4.1697717054,2.6338328667,3.3423071312$ H,4.6779119899,3.3249963591,4.0095396796 C, $4.9204701558,1.7845809721,2.5224764561$ H,6.006882732,1.8040423885,2.5482514474 C,4.242195996,0.9173894101,1.6625145565 C,4.752058831,-0.0645841181,0.6902171463 C,6.016566911,-0.6160845535,0.4452101845 H,6.8847201719,-0.2766615612,1.0040309727 C,6.157087134,-1.6689374837,-0.4927986645 H, 7.1450559327,-2.0852803731,-0.6667044297 C, $5.0793155041,-2.1378038977,-1.2206296729$ H,5.2270733287,-2.8681522601,-2.0100761544 C. $3.7544746118,-1.5775875061,-1.0290696286$ H,3.3073797108,-1.3190538047,-1.9927150507 C,3.6603542426,-0.6034420167,0.0150709771 C, $1.1314757637,-1.5512217094,-0.8792056305$ C, $2.1981523854,-2.5727178215,-0.6182400866$ $\mathrm{H}, 2.4429623388,-2.7387207563,0.4292462986$ C,2.1698595504,-3.8388342017,-1.3679505806 C,3.1517515412,-4.8091714152,-1.0971284412 H,3.9087990782,-4.5947960559,-0.3461165589 C,3.1850077735,-6.0115506117,-1.7913751973 H,3.95744495264,-6.7453016971,-1.5786748156 C, 2.219058143,-6.2628546862,-2.7808427984 H,2.2482555406,-7.1909625258,-3.3457894329 C, $1.2182485528,-5.3342276703,-3.0381393974$ $\mathrm{H}, 0.483911928,-5.5504094345,-3.8033439213$ C, $1.1551362569,-4.1131684573,-2.3304522938$ C, $0.0698514437,-3.1669847301,-2.4611739485$ C, $0.1132613536,-1.927434233,-1.8139692985$ C, $-1.1773929775,-1.2690734725,-1.9604293822$ C, $-2.0163564663,-2.1868508366,-2.655122895$ C,-1.2729409871,-3.331511015,-3.0468863075 C,-1.9361927297,-4.2985010477,-3.8006920122 $\mathrm{H},-1.4537009758,-5.2047676552,-4.1478481417$ C,-3.2964328708,-4.0904282479,-4.1254743185 H,-3.8096180832,-4.847094821,-4.7134503985 C,-4.0061329523,-2.9617683491,-3.7151925781 H,-5.0530215914,-2.8680704987,-3.9861293951 C,-3.360113899,-1.9620742196,-2.9494982428 C,-3.9488640174,-0.7629276478,-2.3636422787 C,-5.3394028598,-0.5232473752,-2.4110926844 H, $-5.9805807243,-1.217678441,-2.9453883891$ C, $-5.9097260516,0.5618805924,-1.7628908574$ $\mathrm{H},-6.984443566,0.7177685361,-1.8084366813$ C, $-5.0977294874,1.4367303941,-1.0204777888$ $\mathrm{H},-5.5421608905,2.2618297813,-0.4700784493$ C,-3.7258683285, 1.2427967268,-0.980774214 H,-3.118209317,1.9160123291,-0.3860932657 C,-3.1026199358,0.1708828861,-1.6685289446 C,-1.6451494728,-0.0216943217,-1.6075528838 
C, $-0.7038900714,1.0943155398,-1.3802139849$ C,-1.0686477404,2.3370489272,-1.895147565 $\mathrm{H},-2.0259694274,2.4415834136,-2.3928452752$ C, $-0.2376791132,3.4770729579,-1.8599414044$ C,- $-0.6341623189,4.7327456925,-2.4005657451$ H,-1.6189238647,4.8254174707,-2.852636471 C, $0.2219366337,5.8124479737,-2.3470850424$ $\mathrm{H},-0.0844246619,6.7704913292,-2.7589983207$ C, $1.5096682759,5.685535057,-1.7557110307$ H,2.1694581071,6.5486157279,-1.7217530994 C, $1.9217259024,4.4796016769,-1.2309294271$ H, 2.9048918271,4.3754020444,-0.7776522539 C, $1.0564945729,3.348499213,-1.270857285$ C, $1.437745884,2.0893934144,-0.7663052872$ H,2.4446378973,2.0159309306,-0.3765155153 C, $0.6241122996,0.9541582587,-0.7987464116$ H,2.1962187671,-1.0642305592,1.4104996651

\section{4-P2-TS1-T1 \\ 11}

C, $0.1115912656,-1.4008844765,-0.5248592835$ C, $0.0846649154,-2.8333145378,0.1036763113$ C,- $-0.8682370412,-2.8777456759,1.3132600153$ C, $-2.2586822795,-2.9271834641,1.3844769338$ $\mathrm{H},-2.8727076309,-2.9572615193,0.4934556706$ C, $-2.8778005084,-2.9388994977,2.6425118533$ $\mathrm{H},-3.9624973583,-2.9776467883,2.6985483781$ C,-2.1215993674,-2.9001868667,3.8210421322 $\mathrm{H},-2.6216966797,-2.9023222403,4.7859043965$ C,-0.7253543852,-2.8759407327,3.7598965941 $\mathrm{H},-0.1279534142,-2.8707934194,4.6678020675$ C,-0.1141408415,-2.8764311689,2.5053146389 C, $1.3077782709,-2.916401045,2.151606724$ C, $2.4495705598,-2.7170932719,2.9448781898$ $\mathrm{H}, 2.345835484,-2.568959996,4.0165631032$ C,3.7239227732,-2.6498880575,2.3472394494 H,4.5977984855,-2.4823040278,2.9696299697 C,3.8611839951,-2.8174458971,0.9846403826 $\mathrm{H}, 4.8453293857,-2.8171161771,0.5256500412$ C, $2.7098584814,-3.0321360281,0.1269296082$ $\mathrm{H}, 2.904788027,-3.8340188249,-0.5905741106$ C, $1.423962039,-3.0703415327,0.7745104685$ C, $-1.1640332483,-0.7881613629,-0.8886407803$ C, $-2.2002592219,-1.5449355057,-1.4279406924$ H,-2.06116201,-2.6046099328,-1.6213721308 C, $-3.4596837973,-0.9728967238,-1.7398160112$ C,-4.49180219,-1.7744551016,-2.3061947957 $\mathrm{H},-4.2762815643,-2.8169865133,-2.5300520064$ C,-5.7400245861,-1.2479661722,-2.5610848019 $\mathrm{H},-6.5192894467,-1.8703332825,-2.9931056929$ C,-6.010393204,0.1099902848,-2.2563533976 H,-6.996784811,0.5199147709,-2.4578819967 C,-5.0327045135,0.9178028789,-1.7097363215 $\mathrm{H},-5.2628235485,1.9534126474,-1.4914857487$ C,-3.7310420113,0.41324066,-1.4413671342 C,-2.6612432149,1.1855205155,-0.904742345 C, $-1.392842498,0.6002647908,-0.7234717789$ C, $-0.4793268922,1.5860258284,-0.1721464165$ C,-1.2562617337,2.7590769196,0.0667322948 C, $-2.581085893,2.5809193855,-0.4125741573$ C,-3.4422013941,3.6700042786,-0.3352825956 $\mathrm{H},-4.4663177423,3.6311076888,-0.6889436498$ C,-2.9577788211,4.8817607265,0.2151940205 H,-3.6349279247,5.7299727436,0.2755484723 C,-1.6497409297,5.0319845019,0.6704973068 $\mathrm{H},-1.3377975484,5.989943077,1.075041792$ C, $-0.745072574,3.9436810082,0.5921398283$ C, $0.6474042069,3.9019314545,1.0253545878$ C, $1.2245319441,4.9684296278,1.7492682288$
$\mathrm{H}, 0.6308207441,5.854889853,1.9500118263$ C, $2.5203377079,4.8980793861,2.236568705$ $\mathrm{H}, 2.936278048,5.7306067872,2.7980942885$ C.3.2793108272,3.7334406615,2.0283541438 H,4.2793423438,3.6494906474,2.4458707158 C, $2.7494183116,2.6815084262,1.2979380203$ H,3.3447340645,1.784411779, 1.1702549396 C, $1.4434515152,2.7333174722,0.7477392902$ C, $0.8863667735,1.5978547299,-0.0059299984$ C, $1.7396410455,0.5803915,-0.6671054458$ C, $2.9506743004,0.9655233206,-1.1873428242$ H,3.2330725862,2.0121697388,-1.1539467217 C,3.8953130916,0.065877155,-1.7944585608 C, $5.0788311179,0.5477202569,-2.3952501721$ H, 5.2736250488, 1.6173602748,-2.393665523 C,5.9827708371,-0.3310776509,-2.9800588235 H,6.8911119332,0.0501864124,-3.4389752065 C,5.7219963858,-1.7126178641,-2.9818151657 H,6.4238125218,-2.3996411083,-3.4465886614 C,4.5589506407,-2.2005850128,-2.3916317733 H,4.358863984,-3.2698582756,-2.3913623299 C,3.6411566729,-1.3240162073,-1.7915983356 C,2.3940589912,-1.8297335459,-1.1789108355 H,1.9271882139,-2.556452974,-1.8397060999 C, $1.3534026048,-0.8322042207,-0.7508818044$ $\mathrm{H},-0.1565703102,-3.5888195797,-0.6572144694$

\section{4-P7-TS1 \\ 11}

C, $0.2572299136,1.4709446121,0.5463699491$ C, $0.8620380016,2.6198578912,1.2261767274$ C, $2.2152089497,2.5727115558,1.6899108833$ C, 3.0627544623,3.6888766942,1.6171349932 H,2.6704855207,4.6501940318,1.2971923407 C, $4.4266579257,3.5174413247,1.8824084752$ H,5.1055880109,4.3601083386,1.7964462454 C,4.9038738211,2.2659851717,2.2750433468 H,5.960085972,2.1380690328,2.4948010596 C,4.0315399558,1.1696349879,2.447134547 H,4.4186762922,0.2320182755,2.8349521652 C, $2.6856195317,1.3143206649,2.1576364488$ C, $1.5838145501,0.3747406175,2.4911820844$ C, $1.6842241166,-0.6040118057,3.4704786338$ $\mathrm{H}, 2.6531257599,-0.8523393328,3.8933257815$ C, $0.5250109402,-1.2256120328,3.9768805782$ H, $0.6201406777,-1.9859250365,4.746932691$ C,- $-0.7325262011,-0.816628422,3.5485162233$ H,-1.6326002693,-1.2387993094,3.9852668396 C, $-0.8478984505,0.1481107608,2.5347942018$ H, $-1.8317746919,0.4489129852,2.1990810841$ C, $0.3044154255,0.6716208703,1.9465016765$ C, $1.1371576501,0.7156798633,-0.4718133774$ C,2.3238693661,1.1921161507,-0.9936117242 H,2.6544796224,2.2065158895,-0.803756268 C,3.1916717497,0.3517762561,-1.7476829205 C,4.4183922732,0.8637263965,-2.2559105546 H,4.6488082978, 1.9151914854,-2.0983802069 C,5.3055350178,0.0474250137,-2.9251850778 H,6.2395264108,0.451590005,-3.3068382472 C,5.0029713895,-1.325327123,-3.1132134786 H,5.7073700214,-1.9637375553,-3.6403986706 C, $3.8225830628,-1.8564845411,-2.6354590714$ H,3.6078620124,-2.9063182768,-2.7948716484 C, 2.8787197597,-1.0427374573,-1.947588889 C, $1.6278494734,-1.5049492098,-1.4547683687$ C, $0.7545333832,-0.6006821856,-0.8142893045$ C, $-0.4784924393,-1.2848268266,-0.4753534132$ C, $-0.2766815557,-2.6611592318,-0.7939824095$ C, $0.9779640286,-2.8386442432,-1.4360483056$ 
C, 1.2881920294,-4.1153947295,-1.8903910496 H,2.2154541441,-4.3434539089,-2.4041761948 C, $0.3479365111,-5.1562809423,-1.6866010801$ $\mathrm{H}, 0.5974565962,-6.1543582709,-2.0377239486$ C, $-0.8853774704,-4.9485126305,-1.0726221662$ $\mathrm{H},-1.5695778636,-5.7843350518,-0.962507639$ C,-1.2384724591,-3.6528291567,-0.616933608 C,-2.481103204,-3.2315354203,0.0236690398 C,-3.4584857497,-4.1649135623,0.4319367209 $\mathrm{H},-3.2945845501,-5.2213469211,0.2425060631$ C,-4.6116024904,-3.76624153,1.0917745283 $\mathrm{H},-5.3430226758,-4.5078752536,1.4022801484$ C, $-4.8181605212,-2.4053215765,1.3782877779$ $\mathrm{H},-5.7023448319,-2.0892232821,1.9257322497$ C,-3.8875897022,-1.4627766636,0.9688605466 $\mathrm{H},-4.0521138646,-0.4185269727,1.2135092052$ C,-2.7133358825,-1.8312528818,0.267502496 C, $-1.7229734784,-0.8203031595,-0.119386385$ C,-2.0577059436,0.6169595112,-0.2998427338 C, $-3.2755297481,0.9190099237,-0.8989779044$ H,-3.9615078673,0.113845427,-1.1416880018 C,-3.6532180596,2.2317251799,-1.2640349223 C,-4.9031880025,2.5204357956,-1.8817306966 $\mathrm{H},-5.5998686637,1.7057039493,-2.0656860886$ C,-5.2204636974,3.8136392744,-2.240117132 $\mathrm{H},-6.1761178087,4.0293616517,-2.7108939637$ C,-4.3031365662,4.872204549,-2.001650109 $\mathrm{H},-4.5673283846,5.8855679502,-2.2927117109$ C,-3.0834770094,4.6198350288,-1.4083127285 $\mathrm{H},-2.3781094667,5.4276622,-1.2260329378$ C,-2.7318290519,3.2962540041,-1.0247101041 C,-1.4889677476,2.9858981062,-0.4181388561 $\mathrm{H},-0.795450624,3.8114271805,-0.2838104208$ C, $-1.1376009442,1.7032610539,-0.0379671$ H, $0.2684738103,3.5089200531,1.428288036$

\section{4-P3-IN1}

12

C,-1.150380759,-0.3905696202,0.3474456206 C,-2.0927918787,-0.7386250771,-0.5694495702 C,-3.1282654312,0.1555497687,-1.1749989139 C, $-3.3205938678,1.5424791639,-1.1411173253$ H,-2.6409937067,2.1980094947,-0.6136988271 C,-4.4090907521,2.1049404013,-1.8209391776 $\mathrm{H},-4.5518032641,3.1822521197,-1.7925011506$ C, $-5.3074803651,1.301989976,-2.5354463682$ $\mathrm{H},-6.1503339197,1.7561278155,-3.0502864688$ C, $-5.1099968585,-0.081246417,-2.604450033$ H, $-5.7855523683,-0.709835091,-3.1790901308$ C, $-4.0193498615,-0.6396805041,-1.9380206785$ C,-3.5408706982,-2.0235214943,-1.9282638461 C, $-4.0460050023,-3.1426692919,-2.5906077817$ $\mathrm{H},-4.9607346044,-3.0714694865,-3.1737324837$ C, $-3.3435297286,-4.349522429,-2.5098283745$ H,-3.7191686322,-5.231494164,-3.022425126 C,-2.1440204755,-4.4167564426,-1.7891395442 H,-1.5858923972,-5.348966346,-1.753844817 C,-1.6443999136,-3.2958872307,-1.1134082705 H, $-0.7064233044,-3.3840293782,-0.5823358253$ C,-2.3547124801,-2.0902494848,-1.1556667454 C, $-0.139241687,-1.323334438,0.9097232129$ C, $-0.404268283,-2.4375747813,1.6734190841$ $\mathrm{H},-1.4261314455,-2.6780527509,1.9530534028$ C, $0.6388613517,-3.3385664196,2.0655495532$ C, $0.3350863591,-4.473867167,2.8456805761$ H,-0.691443045,-4.6306738969,3.1666024979 C, $1.324652983,-5.3831848432,3.2011106341$ H,1.0697439103,-6.2500054683,3.804511021 C,2.6579357965,-5.1866113689,2.78376725
H,3.4256899024,-5.8988626618,3.0720633747 C,2.991779407,-4.0872718788,2.0135001932 H,4.0211535497,-3.94129578,1.7106488155 C, $2.0039758876,-3.1360355945,1.6307488866$ C, $2.2764488653,-1.9824969786,0.8580777486$ C, $1.2161745448,-1.0524397507,0.5997163619$ C, $1.738613694,0.0727943408,-0.1026912759$ C,3.1134356019,-0.2237358009,-0.3972346181 C,3.4916307057,-1.4560868203,0.1942004427 C, $4.807901563,-1.8674850556,0.0410276694$ H,5.1837851224,-2.7955367613,0.4564648055 C,5.6958835297,-1.0362533294,-0.6931310892 H, $6.7251223267,-1.3615035707,-0.8165025216$ C,5.3011923195,0.1734255502,-1.249588354 H,6.0273400896,0.7690034989,-1.7933300662 C,3.9585848799,0.621824599,-1.097754005 C,3.3600742122,1.8537387848,-1.615560677 C,4.0678869766,2.7155588974,-2.4687412952 H,5.0945675475,2.484157387,-2.7336976384 C, 3.4743903295,3.8581014574,-3.0033526542 H,4.0465011834,4.4989317423,-3.668667576 C,2.1378411908,4.1615729789,-2.7094561235 H,1.6594208834,5.0267970041,-3.1592702609 C, $1.4178358675,3.3397622822,-1.8536499647$ H, $0.3721438619,3.5577425693,-1.6646964367$ C, 2.0042910282,2.1921434589,-1.2603123696 C, $1.2226966151,1.3522231345,-0.36276316$ C, $0.0904115908,1.8501782951,0.426647513$ C, $0.1569163905,3.1559024886,0.933615921$ $\mathrm{H}, 0.9958254065,3.7914495238,0.6708544314$ C, $-0.7927399878,3.6620675996,1.8471272681$ C, $-0.7120742627,4.9901422606,2.3536617454$ H,0.1002955109,5.6319091151,2.0212757971 C,-1.6565859348,5.4524099466,3.249252632 H,-1.5942896668,6.4673622842,3.6320268711 C,-2.7097405828,4.6064630289,3.6753704407 $\mathrm{H},-3.4456234942,4.9817516765,4.3814550887$ C, $-2.8072933756,3.307076862,3.2040831283$ $\mathrm{H},-3.6147944911,2.658533556,3.534624568$ C,-1.8540936221,2.8072431857,2.2851037383 C,-1.9115558297,1.4820387728,1.7718119973 H,-2.7314282602,0.8392614202,2.0822740318 C, $-0.9926077467,1.0035331329,0.8627082214$

\section{4-P3-TS-T1}

12

C, $0.4211969622,1.0487448986,0.1196790736$ C, $0.3174955272,2.1942994836,-0.6427312731$ C, $1.5243060679,2.894298722,-1.0291973285$ C, $2.8958899742,2.4485811912,-0.8132968135$ H,3.1902209042,1.5993035689,-1.4449130808 C,3.9042818233,3.5072194052,-0.9254093763 $\mathrm{H}, 4.9442106504,3.2378684539,-0.7686459123$ C,3.5667039065,4.789749693,-1.2876962888 H,4.3401044134,5.5458786485,-1.3867334299 C,2.2191260264,5.147298191,-1.5667971431 H,1.9839052757,6.1706225001,-1.8470239839 C,1.204459471,4.2012444664,-1.433457964 C, $-0.2595155241,4.2941335638,-1.5199409153$ C, $-1.0777273335,5.3243838574,-1.9792757911$ H, $-0.6491454327,6.260785931,-2.327090831$ C, $-2.4624461153,5.11974516,-2.0008605823$ H,-3.1184332726,5.9081034257,-2.3602872662 C, $-3.0082203307,3.8993701872,-1.5763514168$ $\mathrm{H},-4.0837928781,3.748843693,-1.6165242106$ C,-2.1902039186,2.8681315773,-1.0986220809 H,-2.6327140958,1.9324776258,-0.7759171856 C, $-0.8082655723,3.0674402872,-1.0527398809$ C,-0.6954404989,0.3913412229,0.8094520054 
C,-1.6689965172,1.1003952021,1.502778679 $\mathrm{H},-1.5847794577,2.1779404304,1.6056759405$ C,-2.7903218064,0.4500713739,2.0725988313 C,-3.7733331468,1.2011886669,2.7803592987 $\mathrm{H},-3.6175879775,2.2702165942,2.9067880012$ C,-4.9007255816,0.5922541998,3.2856835002 $\mathrm{H},-5.6455407473,1.1752461158,3.8207992332$ C,-5.0943569515,-0.8018765003,3.1045900128 H,-5.9868226402,-1.2756041667,3.5054662198 C,-4.1628709449,-1.5619717464,2.4273551704 H,-4.3298067489,-2.6250659167,2.3047408186 C, $-2.9830384555,-0.9718551337,1.8955622379$ C,-1.9741660847,-1.6905609324,1.1965098468 C, $-0.8204251063,-1.0184144904,0.7430916566$ C, $0.0413188568,-1.9561743262,0.0410618943$ C, $-0.6694272163,-3.1946136641,0.0013282303$ C, $-1.8763190376,-3.0984816089,0.7431905824$ C,-2.6401756673,-4.2520917457,0.8790430991 $\mathrm{H},-3.5673692291,-4.2752516093,1.4413003569$ C,-2.1808060769,-5.4460380048,0.270369785 H,-2.7816372948,-6.3456636646,0.3769306511 C, $-0.9904640315,-5.5137161144,-0.449511123$ H, $-0.6905219998,-6.4604150644,-0.887964988$ C, $-0.1836633778,-4.3566071188,-0.5938222302$ C, $1.0752909425,-4.226273363,-1.3197250706$ C, $1.5742921879,-5.2722859401,-2.1265920363$ H, 1.0281526327,-6.2092857542,-2.1789930614 C, $2.7297702106,-5.1211384004,-2.8780598068$ H,3.0860131763,-5.9402803609,-3.4972115403 C,3.4181777781,-3.8957079654,-2.8601195551 H,4.2998988769,-3.7541516254,-3.4796445784 C,2.9651592191,-2.857822874,-2.0608129737 H,3.4947219583,-1.9112827459,-2.0853726695 C, $1.8107769746,-2.9893926334,-1.2479598133$ C, $1.3354835169,-1.8733271002,-0.4204733572$ C, $2.2302738346,-0.7798918325,0.0459477802$ C,3.5703984702,-1.1166095498,0.3535642849 H,3.894967626,-2.1430376191,0.2263330826 C,4.4942790464,-0.2116282388,0.9413479162 C,5.7799513773,-0.640738927,1.3634359653 H,6.0759358155,-1.6717767768,1.1867309126 C,6.6384666225,0.2412520405, 1.9990921005 H,7.6211410444,-0.0930883569,2.3206893327 C, $6.2375899847,1.5717822756,2.2393897402$ H,6.9101290169,2.2566167913,2.7484873935 C,4.9815553417,2.0098613911,1.8276577182 $\mathrm{H}, 4.6745517923,3.0374470042,2.0073062654$ C,4.1055607433,1.1351917424,1.1703317401 C, $2.7844607425,1.585286387,0.6919592339$ H,2.3594520054,2.3469294429,1.3483196728 C, $1.7910760629,0.52404337,0.3337683142$

\section{4-P3-TS-C1}

12

C,-1.0711751261,-0.1329736533,0.1120493978 C, $-2.0953946186,-0.5761810034,-0.6804719723$ C, $-3.3098681801,0.0646227717,-1.2305603482$ C,-3.6752015082,1.4009846284,-1.400952046 H,-3.0063582103,2.2050679999,-1.1141430305 C,-4.9271196464,1.69406809,-1.9588579014 H,-5.2176077126,2.7314860757,-2.1023349228 C, $-5.8070878187,0.6687095697,-2.3310206524$ H,-6.7763933274,0.9188729072,-2.7544665776 C,-5.4465005344,-0.6770439441,-2.171386816 H,-6.1252221414,-1.4714571926,-2.4710895071 C,-4.1968536823,-0.9736471789,-1.6339998615 C, $-3.5334714302,-2.2703981602,-1.3983546561$ C,-3.9842788398,-3.5958983858,-1.4876897925 $\mathrm{H},-4.9927424071,-3.826954368,-1.8177296943$
C,-3.0999822992,-4.6392175993,-1.1025745062 H,-3.4493349411,-5.6669614732,-1.1640153135 C,-1.8032175603,-4.3964321855,-0.7000269206 H,-1.138184004,-5.2251703093,-0.4778945664 C,-1.2566994432,-3.0323731369,-0.64161071 H,-0.3204746949,-2.9533690831,-1.2086304444 C,-2.2405048318,-2.0001201363,-0.9540256903 C, $-0.1105244689,-1.1590346113,0.5745433152$ C, $-0.5891653614,-2.5078201503,0.9260264387$ H,-1.4893645283,-2.5060269376,1.5414995412 C, $0.4212413754,-3.4756735195,1.3893679813$ C,0.0074876289,-4.6545879285,2.0311001351 H,-1.0501884239,-4.8015607497,2.2333758587 C,0.9297655637,-5.6277652351,2.3945962063 H,0.5943424397,-6.5334926599,2.8923705587 C,2.2997213073,-5.442779138,2.1199207405 H,3.0180104318,-6.2053189044,2.407699318 C,2.7355749293,-4.2859546851,1.4986281225 H,3.7945040547,-4.1445728089,1.325133484 C, $1.8151852078,-3.2704583171,1.1280040388$ C,2.2070372424,-2.0116678701,0.5747876269 C, $1.2379570864,-0.9460888723,0.4498342287$ C, $1.8964979584,0.2350714282,-0.0907708808$ C,3.2275823277,-0.1667925993,-0.3779718391 C,3.4639944011,-1.5091691687,0.0459904847 C,4.7509820047,-2.0328341434,-0.1365665622 H,5.0128775245,-3.0440720314,0.15144829 C,5.7336962212,-1.2102727285,-0.711530314 H,6.7333785252,-1.6113611609,-0.8527047497 C,5.4764826982,0.1087584889,-1.1080603156 H,6.2847520436,0.6869289893,-1.5452092766 C,4.191225856,0.6711856937,-0.9439193138 C,3.7375761403,2.0016616583,-1.3323127529 C,4.5736394279,2.8829637994,-2.0511057268 H,5.5911641731,2.5830422109,-2.280739299 C,4.1165551398,4.1142514331,-2.4944003318 H,4.779244943,4.7699946376,-3.0529533565 C,2.786393671,4.4953216756,-2.2468039598 H,2.4072463177,5.4387678867,-2.6304037208 C,1.9500328873,3.6630206176,-1.5205434237 H,0.9211619399,3.9647977393,-1.3612377632 C,2.3945241192,2.4153484818,-1.0138520056 C, $1.4896060006,1.5425658973,-0.2557239347$ C, $0.2749020023,2.0469752669,0.435062745$ C,0.3211746489,3.299882639,1.0363566553 H, $1.2250623479,3.8959643529,0.9642806455$ C, $-0.7570792893,3.838279813,1.780408763$ C,-0.6957249272,5.1295403717,2.3750267012 H,0.2099885223,5.720316701,2.2587725471 C,-1.7730899832,5.623997109,3.0814052913 H,-1.7217689549,6.6124945593,3.5304392137 C,-2.9544885913,4.8496358845,3.2281938307 $\mathrm{H},-3.7943781044,5.2550187565,3.7865063737$ C,-3.0389279946,3.5904923613,2.6693537207 H,-3.9401037787,2.9919074312,2.780844449 C, $-1.9444563094,3.0569035549,1.9356308604$ C,-1.9765580237,1.7665614243,1.3530174859 H,-2.8680151279,1.1600235863,1.4864075788 C,-0.9187522843,1.2540915379,0.6221358283

\section{4-P3-IN2}

12

C, $-1.071728329,-0.1593668176,0.1654913964$ C,-2.2437690138,-0.6111443011,-0.4907567781 C,-3.4299191052,0.061201992,-1.0863393422 C,-3.796246884,1.4022607288,-1.2260091359 H,-3.1715126046,2.2008164424,-0.8444288138 C,-4.9953688449,1.7173747242,-1.8820768119 H,-5.2780792932,2.7607816,-1.9951337678 
C,-5.8293290346,0.7141505101,-2.3907548841 H,-6.756915784,0.982335704,-2.8894196444 C,-5.4678663535,-0.634972439,-2.2709909048 H,-6.1025010677,-1.4171426867,-2.6796206015 C,-4.2719663895,-0.9517995678,-1.6343023344 C,-3.6197147426,-2.2492991784,-1.4145284621 C,-4.0039327846,-3.554039437,-1.6164929334 $\mathrm{H},-4.9649683125,-3.7918612301,-2.0651142145$ C, $-3.1425333693,-4.6326574494,-1.1886725017$ $\mathrm{H},-3.4868065369,-5.651948387,-1.3407402953$ C,-1.9319949945,-4.4098100919,-0.6098412643 $\mathrm{H},-1.295028806,-5.2450720165,-0.3387884932$ C, $-1.3674263763,-3.0233965557,-0.4895096066$ H,-0.6197170494,-2.9722587932,-1.3121904637 C, $-2.3778388785,-1.9778753435,-0.7787107606$ C, $-0.1168518364,-1.1619324556,0.5308059128$ C,-0.6098700578,-2.5830694954,0.8054096951 H,-1.3735762314,-2.515210821,1.5941441484 C, $0.4531885253,-3.5467042497,1.2882505888$ C, $0.0585943068,-4.7361044549,1.9075317078$ $\mathrm{H},-1.000681666,-4.9347872295,2.0490071245$ C, $1.0006052607,-5.6654933326,2.3552553284$ $\mathrm{H}, 0.6696032814,-6.5821885933,2.8356769346$ C,2.3670499995,-5.4025633426,2.1991812732 H,3.10633065,-6.1141758257,2.5564988722 C,2.7809884826,-4.2118782919,1.6121639254 H,3.8397639697,-3.9928038188,1.5529589508 C, $1.8399463472,-3.2649942003,1.1493534822$ C. 2.2198413244,-1.9772007746,0.6176071536 C, $1.2540973233,-0.9249185486,0.4971959799$ C, $1.922497975,0.2566020479,-0.0407709006$ C,3.256237649,-0.1424921084,-0.3240894857 C,3.4842256501,-1.4836599148,0.0999136198 C,4.7627606048,-2.0200983168,-0.0986268933 H,5.0148392756,-3.0394056856,0.1697327324 C,5.7497544609,-1.2032686109,-0.6795246126 H,6.7438430724,-1.6149396899,-0.8310740781 C,5.5011936533,0.1157980958,-1.0754391713 H,6.3095738981,0.6888149031,-1.518440992 C,4.217125385,0.683988983,-0.9081305154 C,3.7625354635,2.0057518689,-1.3231046896 C, $4.5956648762,2.8749355802,-2.0603686764$ H,5.6135645316,2.5726073985,-2.286425759 C,4.1337347383,4.0950265873,-2.5292875624 H,4.7942898105,4.7418236112,-3.1006210541 C, 2.8015705066,4.4759640144,-2.291292008 H,2.4194796364,5.4099871286,-2.6944976119 C, $1.9673185585,3.6544468078,-1.550531855$ H,0.9362511192,3.9532917382,- 1.400267916 C. 2.4172544385,2.4194855126,-1.0176294862 C, $1.513973831,1.5569233392,-0.2473224967$ C,0.2900784349,2.0555642878,0.4134561926 C,0.3189569881,3.3194153738,0.9951029909 H, 1.2190431866,3.9202613719,0.9187833491 C, $-0.7598330372,3.8551607373,1.7346485882$ C,-0.7086546663,5.1498193713,2.3243689997 H,0.1896122827,5.7501661858,2.199833261 C,-1.7857409378,5.6323761849,3.0376504083 $\mathrm{H},-1.7432848934,6.6225807091,3.4839165289$ C,-2.9576102103,4.8434583887,3.197050971 $\mathrm{H},-3.7971448648,5.2413639635,3.7611808368$ C,-3.0320637079,3.5823507509,2.6441259169 H,-3.9249534706,2.9732842809,2.7646434754 C,-1.9360826055,3.0595085185,1.9016108206 C,-1.9579989016,1.7687745377,1.3275780011 $\mathrm{H},-2.840982392,1.1575129288,1.4881618536$ C, $-0.895012464,1.2475571469,0.5988527578$

\section{4-P4-IN1}

21

C,-0.9870091102,-0.0308415,0.0908379822

C,-2.3947151886,-0.1986042893,-0.3570404842 C, $-2.7937899191,-0.1743873751,-1.7307136626$ C,-2.0270861348,0.0094417158,-2.8912292195 H,-0.9572472762,0.1864812449,-2.8291124891 C,-2.6830688652,-0.0406847989,-4.1271696037 H,-2.1216851219,0.0991529297,-5.0452978949 C,-4.0606712644,-0.2710979236,-4.1819369718 $\mathrm{H},-4.5543946732,-0.30968665,-5.1487108672$ C,-4.8414802164,-0.457136591,-3.0086189774 H,-5.909758691,-0.6352522713,-3.0859482035 C, $-4.2044136732,-0.4036942843,-1.7919530175$ C,-4.6876553642,-0.5436222257,-0.3977209498 C,-5.9354926271,-0.7353623872,0.145743922 H,-6.8205129012,-0.8303542071,-0.4760128562 C,-6.0450290224,-0.802015858,1.5616577635 H,-7.0269191291,-0.9531425717,2.001511519 C,-4.9342092183,-0.6731403428,2.4009872397 H,-5.0610986814,-0.7250213204,3.4774779953 C,-3.6605930151,-0.4761559182,1.8538301395 H,-2.7828185093,-0.3735865374,2.4855899124 C, $-3.5489090177,-0.4182893274,0.4573812515$ C,-0.2871939575,-1.2448686443,0.3480805449 C,-1.0179793366,-2.452277262,0.5311708843 $\mathrm{H},-2.0995586551,-2.4531575367,0.5056332885$ C, $-0.3945421903,-3.6704852513,0.8013439868$ C,-1.1916718413,-4.8382702397,1.0309694688 H,-2.2737850049,-4.7415440811,1.0022553462 C, $-0.5977452168,-6.0493843108,1.2831452595$ H,-1.2004499992,-6.9354904719,1.4585866221 C,0.8199314957,-6.1425004407,1.3191138983 H,1.283920892,-7.1033508031,1.5252123687 C,1.6218155516,-5.0385021911,1.1004743348 H,2.6961426029,-5.1525337819,1.1466741387 C, $1.0515248867,-3.7684148088,0.8353456021$ C, $1.7952457513,-2.58157485,0.5890163207$ C, $1.1376467405,-1.3431605896,0.4042667779$ C, $2.1189427689,-0.3330043131,0.0835613285$ C,3.3829429026,-0.9937068203,0.0419985518 C, 3.2424036091,-2.3572393812,0.3998526984 C,4.3993031358,-3.127440399,0.4789828342 H,4.3904051979,-4.1754041995,0.753670074 C,5.6404142836,-2.5126000368,0.1973448754 H,6.5429132057,-3.1144800766,0.2608175041 C,5.7511553938,-1.1701612505,-0.1616953256 H,6.7336107923,-0.7601384419,-0.3723548259 C,4.5916353019,-0.3635582016,-0.2490172152 C,4.5045215978, 1.0290344689,-0.6720764597 C,5.6306653612,1.7162424005,-1.1719548786 H,6.5950239813,1.2190018475,-1.1831212636 C,5.5274576647,3.0018664236,-1.6809090211 H,6.4098201724,3.5049772448,-2.0671549978 C,4.2734227178,3.6334733147,-1.7304127872 H,4.1733555262,4.6187408052,-2.1774981073 C,3.1557873655,2.9957062719,-1.2174597769 H,2.1956539272,3.4928059967,-1.291710272 C, $3.2323003818,1.7025436101,-0.6373904905$ C, $2.0373665174,1.0362558074,-0.0982298737$ C, $0.8554564361,1.7942555375,0.3323986026$ C, $1.0561148608,3.0617249943,0.8618191779$ H,2.0701514712,3.3995999886,1.0426604693 C,0.0107348267,3.9593384662,1.1748366799 C,0.2515036001,5.2439996465,1.723894894 H, 1.2721730796,5.5426871249, 1.9473818438 C,-0.8041212758,6.1025243747,1.9606287657 H,-0.6146585373,7.0872505602,2.3792127044 C,-2.1431383854,5.719928604,1.6652611385 H,-2.9528811967,6.4162048777,1.8629615849 C,-2.4101481431,4.476627563,1.1417954547 
$\mathrm{H},-3.4287251854,4.1670029658,0.9224830007$ C,-1.3387919171,3.5673650003,0.8850572135 C,-1.5656069332,2.2723714933,0.4156443892 $\mathrm{H},-2.6023134102,1.9808841709,0.2927621421$ C, $-0.529937194,1.3182283134,0.2215609973$

\section{4-P4-IN2 \\ 21}

C,-1.0545090137,-0.124609454,0.3180406842 C, $-1.8014815294,0.0030949717,1.4817427114$ C,-2.4068859605,-0.9460004284,2.4440311839 C, $-2.3560700602,-2.3365417787,2.5558779254$ H,-1.8110638097,-2.9397713705,1.8395669391 C,-3.0246868796,-2.9527034122,3.6222614237 H,-2.9855879526,-4.0342097519,3.7184773375 C,-3.7430509569,-2.1989647754,4.5592397611 $\mathrm{H},-4.2608383322,-2.7010754176,5.3717600888$ C,-3.7951154807,-0.8012622082,4.4623277592 $\mathrm{H},-4.340823781,-0.2132973289,5.1952209194$ C, $-3.1213534603,-0.1854427854,3.4131212489$ C, $-2.9578459587,1.2383782455,3.0822263642$ C, $-3.5166535529,2.4094195594,3.575409598$ H,-4.2089975448,2.3784296805,4.4127027928 C, $-3.21742844,3.6698354961,2.9694082373$ $\mathrm{H},-3.6789783854,4.5629967494,3.3789574092$ C,-2.3684002464,3.7698377505,1.9022904497 H,-2.1197218978,4.7406342547,1.4879533035 C, $-1.640831175,2.578168565,1.3884181559$ H, $-0.6113021756,2.7331098297,1.7841488746$ C,-2.1062962111,1.3124456057,1.9668136202 C, $-0.5784675917,1.1216679305,-0.2821310656$ C, $-1.437074155,2.3638073238,-0.1735679478$ $\mathrm{H},-2.4338865912,2.1321897446,-0.5700672876$ C,-0.9091549804,3.5728210257,-0.8958276862 C,-1.7738362032,4.6338998829,-1.172931259 H,-2.8209958287,4.5558126473,-0.8923674332 C,-1.3096590042,5.7867824425,-1.8068586112 H,-1.998925129,6.6001793954,-2.0151964073 C, $0.0370251586,5.8915393267,-2.1890605694$ H, $0.391996763,6.7839239,-2.6958240669$ C, $0.9091479579,4.8425393243,-1.9440476912$ H,1.9304932178,4.9074477222,-2.2967258209 C, $0.4571393839,3.6660565565,-1.2971731995$ C, $1.2878741409,2.5143097465,-1.1142716175$ C, $0.6881408672,1.2228052738,-0.7868565305$ C, $1.7421407082,0.2211031426,-0.7608963947$ C, 2.9512209213,0.9311401106,-0.9516348844 C, $2.707267879,2.3192053426,-1.1945529491$ C,3.8179594208,3.1578772719,-1.4089344636 H,3.7167554268,4.2249055861,-1.5653305236 C,5.0905799309,2.5806426622,-1.4077430152 H,5.9555533679,3.2147459145,-1.5776896735 C,5.2977862808,1.2061525978,-1.18871456 H,6.3161998647,0.8313077598,-1.2079485059 C,4.2160763076,0.3372572227,-0.9407977496 C,4.2586266484,-1.08637744874,-0.6299689084 C, $5.4823738955,-1.7621275065,-0.4446740202$ H,6.4157965027,-1.2237357208,-0.5746826158 C,5.5196591218,-3.0966691927,-0.0703518077 H,6.4757271724,-3.5930626086,0.0715577172 C,4.3206589198,-3.7942077424,0.155279523 H,4.344351878,-4.8270381563,0.4917312046 C,3.103024374,-3.1627318352,-0.0378563218 H,2.1911031544,-3.7098147358,0.1703067877 C,3.0242383404,-1.8119624104,-0.4624441694 C, $1.7275952297,-1.1544329301,-0.6487942085$ C, $0.4607961963,-1.8935286514,-0.8400003573$ C, $0.4752263869,-3.0652245594,-1.5869095071$ H, 1.412199018,-3.4226541973,-2.0005258676
C, $-0.6921344118,-3.8121171041,-1.8777083247$ C, $-0.6531261691,-5.0081829094,-2.6441849606$ $\mathrm{H}, 0.3022018001,-5.3611742527,-3.0242720879$ C,-1.8147392789,-5.710802772,-2.8949742139 $\mathrm{H},-1.7799083887,-6.6262766575,-3.4794292341$ C, $-3.0613386533,-5.24887556,-2.3958727028$ $\mathrm{H},-3.9642844761,-5.8166242408,-2.6035176471$ C,-3.1305804664,-4.0871073175,-1.6549995186 H,-4.0821015479,-3.7261354178,-1.2724421885 C,-1.9488468015,-3.3452646765,-1.3800272899 C,-1.9689318984,-2.145852393,-0.6341089425 H,-2.929641214,-1.778187529,-0.285476469 C,-0.8197666415,-1.4109422856,-0.3677307439

\subsection{Calculations B3LYP-D3/6-311+G(2d) level}

2-bb-gas

C, $0.6572034074,5.1992821256,-2.5816551721$ C, $1.9018000406,5.2051940369,-1.9134702465$ C, $2.2132617172,4.2120139763,-1.0195915791$ C, $1.2943704854,3.169423635,-0.7472690604$ C, $0.0358024642,3.1634465057,-1.4229525307$ C,- $-0.2525872297,4.2002998789,-2.3434311405$ C, $1.5861377577,2.1279446376,0.1636966004$ C, $0.6709514767,1.1424497299,0.4347094503$ C,-0.5899489593,1.1364313517,-0.2422793231 C,-0.8769784355,2.1162251062,-1.1587006083 C, $0.9562996628,-0.049438105,1.2757942294$ C, $0.6727894827,-1.2542765539,0.4527234485$ C, $-0.5880997721,-1.2603420226,-0.2242823936$ C, $-1.4397530423,-0.0609420253,-0.0107928507$ C, $1.5895082638,-2.2422919904,0.1964598195$ C, $1.2993512562,-3.2977578449,-0.6987869321$ C, $0.0407741977,-3.30385839,-1.3744517804$ C,-0.8736191017,-2.2542041322,-1.1259081456 C,2.2198586402,-4.3428850759,-0.9554834898 C, $1.9099314467,-5.3498342564,-1.8343646798$ C, $0.6653249966,-5.3558649385,-2.502530884$ C, $-0.2460102205,-4.3548343151,-2.2792825533$ C, $-2.7375679603,-0.0590376207,0.3777984432$ C, $1.349080587,-0.0393358699,2.57233053$ C, $-3.6154413265,-1.2399165204,0.6230214593$ C, $-4.940810607,-0.7873187166,0.7980940245$ C, $-4.9419708303,0.6719821938,0.7871033933$ C,-3.6173245591,1.1240032381,0.605182629 C, $1.6381837093,-1.2145024829,3.4445222139$ C,2.2210077026,-0.7525927066,4.6441342489 C,2.2199630169,0.7066997196,4.6329986916 C, $1.6364475163,1.1493994963,3.4265069121$ C, $-5.9876865477,1.5593177559,1.0056291821$ C,-5.7116926741,2.9201027573,1.0839270337 C, $-4.3987877888,3.373579238,0.9667822109$ C, $-3.3514975822,2.4863427328,0.7301245627$ C, $-3.3474417138,-2.5997900898,0.7685075485$ C, $-4.3933175687,-3.4850257843,1.0185297612$ C, $-5.7069456343,-3.0319282608,1.1288330021$ C, $-5.9851100125,-1.6729222298,1.0300122573$ C, $1.3787979405,-2.5769023081,3.3072502561$ C, $1.7546273303,-3.4551619439,4.3208689356$ C, $2.3850435387,-2.9925955488,5.4748558525$ C, $2.6110858407,-1.6311637932,5.6464287613$ C,2.6087483088, 1.6010338444,5.6217628814 C,2.3807467896,2.9593611299,5.4294203461 C, $1.7496944753,3.4033491181,4.2685038398$ C, $1.3751293396,2.5091704597,3.2684265824$ H,0.4241198524,5.9890290557,-3.2857073129 
H,2.6115706886,5.9994188157,-2.1113342103 H,3.1683633724,4.2142080899,-0.5063303205 $\mathrm{H},-1.2080866251,4.1934212055,-2.8559101784$ $\mathrm{H}, 2.5504929274,2.1145850536,0.6571268245$ H,-1.8209245131,2.093764089,-1.6898275933 H,2.5538609916,-2.2200234547,0.6895704959 H,-1.8176053219,-2.2411691323,-1.657278866 H,3.1749613546,-4.3359106099,-0.442266724 H,2.6209304716,-6.1458355266,-2.0203215086 $\mathrm{H}, 0.4334651931,-6.1564322095,-3.194665736$ H,-1.2015154708,-4.3571091535,-2.7917916637 H,-7.0001667774,1.1956920633,1.1357996666 H,-6.5140670446,3.626859928,1.2587522973 H,-4.184332753,4.4308487062,1.0666996672 $\mathrm{H},-2.3457331334,2.869215917,0.6624890929$ H,-2.3410718499,-2.9820396285,0.70660644 $\mathrm{H},-4.177176897,-4.5403238729,1.1343856533$ H,-6.5081897415,-3.737245119,1.3143317441 H,-6.9981657758,-1.3089851215,1.1547178184 H,0.8772169997,-2.9665065209,2.4358484195 H, $1.5463778095,-4.5124902583,4.2091444204$ $\mathrm{H}, 2.6782176248,-3.6925448324,6.2481566973$ H,3.0640595254,-1.2600647832,6.5582317163 H,3.062229148,1.24455224,6.5391285466 $\mathrm{H}, 2.6728922827,3.671457761,6.1919445315$ H, 1.5399472984,4.4585503959,4.1406373825 $\mathrm{H}, 0.8730638281,2.8846986706,2.3911412324$

\section{3-bb-gas}

01

C, $-0.8220395395,0.6523015696,-0.0655126137$ C,-1.7423207922,1.4684918683,-0.6807736953 C, $-3.0616628405,0.9790025858,-0.8977469327$ C, $-3.5358917021,-0.2657109591,-0.4718873429$ C, $-4.8657648203,-0.5705031763,-0.869546979$ $\mathrm{H},-5.2815865334,-1.5494268246,-0.6887510737$ C, $-5.6343036174,0.3518152513,-1.556697443$ H,-6.639517608,0.0739641504,-1.8510865389 C,-5.1480240138,1.6270190085,-1.9070668914 $\mathrm{H},-5.7831122536,2.327000293,-2.4369888328$ C,-3.8441574982,1.9305125634,-1.5869016921 C,-2.9822330694,3.0908933186,-1.8313708013 C,-3.2344407511,4.2794110349,-2.5001837592 $\mathrm{H},-4.2151647828,4.4806688637,-2.9155115859$ C,-2.2038753811,5.2063188175,-2.6421602472 H,-2.385756893,6.1395699819,-3.1615533953 C, $-0.936107782,4.9351388071,-2.1296052342$ H,-0.1402693577,5.6590370222,-2.2578382464 C,-0.6750921993,3.7453939069,-1.451310089 $\mathrm{H}, 0.3150071649,3.5555070356,-1.0617770536$ C,-1.6998310148,2.8203699798,-1.282420953 C,-1.2174463422,-0.7179300553,0.2032498162 C,-2.5765454083,-1.1308433806,0.1796191384 C, $-2.9096535413,-2.4218599427,0.7504927179$ C,-4.2001993678,-2.7193500964,1.2427897377 $\mathrm{H},-4.949348223,-1.9423292262,1.2654239697$ C, $-4.5087288008,-3.953830825,1.7695609865$ H,-5.5023450873,-4.1379650501,2.1600392575 C,-3.5317291762,-4.9575733406, 1.8351288915 $\mathrm{H},-3.775252775,-5.9275614684,2.251605782$ C,-2.2490471562,-4.6874637628,1.4192695914 H,- $1.4809655873,-5.4367698079,1.5432859752$ C, $-1.8985728439,-3.4233823128,0.8990961489$ C, $-0.5643265246,-3.0728562157,0.5507474031$ C,- $-0.2300518781,-1.7319965873,0.3645871178$ C, $1.1737085866,-1.653715154,-0.036980701$ C, $1.6247484623,-2.9978083677,-0.1675533016$ C, $0.615160066,-3.8973154757,0.2439190739$ C,0.9103699494,-5.2489661351,0.2220267634
H, $0.1899804596,-6.0033227895,0.5060379946$ C, $2.1935778878,-5.6545298326,-0.1967828807$ $\mathrm{H}, 2.4231690639,-6.7133720864,-0.2124975558$ C, $3.167507731,-4.7524060483,-0.5926811583$ H,4.132664483,-5.1263158,-0.9086632066 C, $2.8932123794,-3.3674912063,-0.5881061212$ C,3.76031491,-2.2878997755,-1.0281059189 C, $4.9968314048,-2.5363004785,-1.6485260731$ H,5.3355882836,-3.5579733997,-1.7611110551 C,5.7745381991,-1.512296109,-2.1459482 H,6.7193387016,-1.7334430271,-2.6276439805 C,5.3226782999,-0.1915971716,-2.0544221509 H,5.9052312435,0.6145011068,-2.4837798789 C,4.1259879186,0.08557788,-1.4288556296 H,3.7793763919,1.1087600899,-1.3862277764 C,3.3235372113,-0.933263258,-0.8719679136 C, $2.0462571559,-0.6167631262,-0.229119385$ C, $1.7597147039,0.6988964558,0.3749778486$ C, $2.798051228,1.3688918529,1.0006829457$ H,3.7955755327,0.953027824,0.9569645559 C, $2.6171042249,2.549841824,1.7457292562$ C, 3.6913839125,3.2298280999,2.3722823447 H,4.6956146457,2.8340038322,2.2697053645 C,3.4633464775,4.3729909865,3.0944821007 H,4.2895983846,4.8892724958,3.5683640363 C,2.1523793822,4.8864058416,3.228472364 H,1.9892232024,5.7912121829,3.8014938775 C, $1.092614399,4.2443284682,2.6405602825$ $\mathrm{H}, 0.085976475,4.6338677145,2.7418499525$ C,1.2984828996,3.0629717075,1.8867418893 C, $0.2441239187,2.3642486813,1.267754327$ $\mathrm{H},-0.7603386858,2.7487046382,1.3907177494$ C, $0.4291891669,1.2265762615,0.5056103082$

\section{4-bb-gas}

01

C,20.158092584,30.2659143906,8.9555114828 C, 20.3318700142,29.7475688267,10.184712354 C, 19.3471152304,29.718961557,11.2982364818 C, 18.058361671,30.2323740115,11.4161465114 H, 17.5895330118,30.7583820293,10.5987730233 C, 17.3653001328,30.0684124433,12.6138093114 $\mathrm{H}, 16.3631060481,30.4695061222,12.7065012867$ C, $17.9439042883,29.3997382933,13.6915625215$ H, 17.3871171539,29.2802319106,14.6133748458 C, $19.2371523882,28.8956195739,13.5914458499$ $\mathrm{H}, 19.6958923753,28.3893262845,14.4326877255$ C, $19.9330631586,29.062939921,12.4015905696$ C, 21.3009071286,28.6868020606,12.0496715128 C, $22.2795589094,28.0589864496,12.8087041671$ H,22.0699916388,27.7364659741,13.8218800962 C,23.5395136599,27.861009322,12.2519589917 $\mathrm{H}, 24.3158435345,27.3749138738,12.8307978065$ C,23.8107994083,28.2959918751,10.955560655 H,24.7990246137,28.1497532699,10.5361724069 C,22.8291519265,28.9204896644,10.1887572007 H,23.0665756933,29.2523112418,9.1897083617 C,21.5600703194,29.1104390092,10.7288872396 C, $18.9280798108,30.9252293176,8.4399558496$ C, $17.8373152427,30.1918253466,8.0421661929$ H,17.8165088709,29.1247149289,8.2325079208 C, $16.7449567915,30.7950822099,7.3797890004$ C, 15.6019626932,30.0622832969,6.975331524 H,15.5554408568,29.0016566822,7.1958229192 C, $14.5717650525,30.6810222669,6.3146232628$ H,13.7023402792,30.1109409476,6.0097566665 C,14.6363981246,32.062947778,6.0249703217 H,13.8161098597,32.5385098771,5.5008276603 C, 15.7291492726,32.7994922329,6.4040853218 
$\mathrm{H}, 15.780015719,33.8601556066,6.184421999$ C, $16.808766226,32.1913758523,7.0918051639$ C, $17.9412792566,32.9235375132,7.513490798$ $\mathrm{H}, 17.9778621082,33.98679586,7.306230368$ C, $18.9826218379,32.3308628513,8.1914652631$ C,20.1410700157,33.1242798628,8.690696679 C, $19.891191105,34.2717763399,9.5399892725$ C, $18.5966471441,34.5665439591,10.0293180296$ H,17.7810594626,33.8959195267,9.8044219497 C, $18.3521276092,35.678145122,10.8008820579$ H,17.3511688856,35.8724091286,11.1669112499 C, $19.4010513754,36.5503380869,11.1174607548$ H,19.2140907435,37.4362057078,11.7124473329 C,20.6783390781,36.2644076399,10.6938896612 H,21.4755428623,36.9356235049,10.9794287178 C,20.9666170838,35.1169647801,9.9244998661 C, $22.3238420849,34.7436275509,9.5753749016$ C,23.440037769,35.5046058615,9.983672185 H,23.2929798291,36.4354123783,10.5124166963 C, $24.7285967494,35.0847523476,9.7470923356$ H,25.5640286135,35.6892880404,10.0792292579 C, $24.9540302422,33.8621643612,9.1023842629$ $\mathrm{H}, 25.9648269303,33.5030734765,8.9508023134$ C,23.8870138946,33.1114200552,8.6680910309 H,24.0754893758,32.1646885022,8.184687277 C,22.5515983481,33.5400548114,8.855472024 C, $21.4282534852,32.7702875294,8.3594831237$ C,21.6916904952,31.5858973552,7.4942960437 C, 22.4542136523,31.6825928885,6.3521451127 $\mathrm{H}, 22.8455488913,32.6483252391,6.0537595543$ C,22.7625081662,30.5542505523,5.5595159119 C, $23.55676747,30.647119149,4.3895315318$ H,23.9305179732,31.6190173422,4.0869259344 C, 23.8521848948,29.5288570497,3.6529518341 H,24.4614290902,29.6113481432,2.7608900147 C,23.3655618936,28.2629423582,4.05101176 H,23.6068201531,27.3874453011,3.4601349787 C,22.5911650742,28.1404024258,5.1761666014 $\mathrm{H}, 22.2145588478,27.1705216297,5.4814673241$ C,22.2675966456,29.2764731402,5.9582564503 C, $21.4546817258,29.1971005662,7.1109819448$ H,21.0420967079,28.2377111367,7.402071849 C,21.1676177001,30.3093721963,7.86353578

\section{5-bb-gas}

01

C, $1.3583513336,0.406520787,-0.1546164938$ C, $2.6080646548,0.739430633,0.3249953139$ C,3.5827149635,-0.2991622506,0.4099090254 C,3.3956405061,-1.6258890424,0.0032252999 C,4.4749106197,-2.50243619,0.2893445605 H,4.3759544501,-3.5643151668,0.1299065667 C,5.6492001988,-2.0351753775,0.8524963173 H,6.4421480235,-2.7424934831,1.0648068763 C,5.8326448021,-0.6803798435,1.1865933507 H,6.7652797662,-0.3470121488,1.6260919566 C,4.7805211506,0.1829107342,0.9817212639 C,4.5617334128,1.5946074352,1.2995430106 C,5.3913084607,2.5102163469,1.9292614926 H,6.4016330191,2.2348872267,2.2089081564 C,4.8999624124,3.7806120434,2.2193535157 H, 5.5356484764,4.5069169385,2.7113298849 C,3.5850857955,4.1136144817,1.8989549335 H,3.2027001264,5.0946281874,2.154243118 C,2.7492327295,3.2030997685,1.2546866609 H, $1.7303987739,3.4854327806,1.0323417217$ C,3.2357554264,1.9418195908,0.92100078 C, $1.0711688103,-0.9875225801,-0.4011757672$ C, $2.087038023,-1.9659251909,-0.5225081508$
C, $1.751510208,-3.2174235663,-1.1648841147$ C, $2.7094271833,-4.113422418,-1.6974327579$ H,3.7553750759,-3.8531448193,-1.676783682 C,2.337345466,-5.2836405893,-2.3193712534 H,3.0997228764,-5.9343971858,-2.7306724591 C, $0.9808789174,-5.6262107496,-2.4510122451$ H,0.6976190909,-6.5556747556,-2.9296876979 C, $0.0212766909,-4.7508681458,-2.0079384195$ H,-1.0311264263,-4.9733741729,-2.1438705606 C, $0.3773877832,-3.5298567032,-1.3901090398$ C, $-0.6235958587,-2.60407619,-1.0144561782$ H,-1.6593241884,-2.876321483,-1.165111498 C, $-0.3092602091,-1.3661463275,-0.5253465272$ C,-1.3583452387,-0.4065485045,-0.1545828161 C,-3.2357367492,-1.9418441268,0.9210783845 C,-2.7492024802,-3.2031114768,1.254797964 H,-1.7303625479,-3.4854357876,1.0324718667 C,-3.5850513262,-4.1136224069,1.8990770434 H,-3.2026574659,-5.0946261032,2.1543914495 C,-4.8999353835,-3.7806290505,2.2194538899 H,-5.5356185396,-4.5069320109,2.7114369208 C, $-5.3912930458,-2.5102449719,1.9293324109$ H,-6.4016240185,-2.2349233669,2.2089633299 C,-4.5617218652,-1.5946380758,1.2996052199 C,-4.7805220702,-0.1829495355,0.9817552339 C, $-5.8326584937,0.6803353167,1.1865917105$ H,-6.7652999642,0.3469657884,1.6260751309 C, $-5.649229008,2.0351252756,0.8524584201$ H,-6.4421953591,2.7424357627,1.0647251227 C,-4.4749339468,2.5023893746,0.2893212444 $\mathrm{H},-4.3759922674,3.564264066,0.1298319943$ C,-3.3956423787, 1.6258500634,0.0032666439 C, $-3.5827100623,0.2991265225,0.409958809$ C,-2.6080514965,-0.7394593833,0.3250553167 C,-1.0711672431,0.9874961297,-0.4011398064 C, $-2.0870383656,1.9658990313,-0.5224393182$ C, $-1.7515260337,3.2174221557,-1.1647639778$ C,-2.7094669228,4.1134557614,-1.6972067615 H,-3.755418217,3.8531871284,-1.676497316 C,-2.3374078531,5.2837037196,-2.3191029171 H, $-3.0998015141,5.9344902778,-2.730326566$ C,- $-0.9809442145,5.626269809,-2.4507943872$ H, $-0.6977020369,6.55575776,-2.9294337459$ C, $-0.0213238347,4.7509042449,-2.0078021282$ H,1.0310740885,4.9734172522,-2.1437631309 C, $-0.3774116552,3.5298647542,-1.3900150014$ C, $0.6235820209,2.6040696908,-1.0144204407$ H, $1.6593067237,2.8763216835,-1.165089178$ C, $0.3092584513,1.3661239149,-0.5253399134$

\section{6-bb-gas}

01

C, $1.419627532,-0.2172345406,-0.1440747253$ C, $2.7101549774,-0.1733772548,0.3478735288$ C,3.6581001159,-1.1521786638,0.9305812685 C, $3.5533821213,-2.5037425459,1.2466177107$ H,2.6551847759,-3.061068402,1.0225479381 C, $4.6187248965,-3.1463446338,1.8757503256$ H,4.5342739058,-4.1990461605,2.1177142585 C,5.7848694305,-2.4555282156,2.1997758775 H,6.6039663855,-2.9762504523,2.6809982018 C,5.891204574,-1.0934386261,1.9280907309 H,6.7821752056,-0.5448764507,2.2110496497 C, $4.8317281857,-0.4444456862,1.312487994$ C, $4.6335657513,0.9749574458,1.0131922661$ C,5.3873945495,2.1064648286,1.2201005609 H,6.3809592463,2.0580258191,1.6499002831 C,4.815281043,3.354965184,0.8970317047 H,5.3753928082,4.2587487326,1.105820919 
C,3.550800586,3.4674136134,0.3510396066 H,3.1448765425,4.4543649645,0.1884345123 C, $2.7698292347,2.3131529334,0.0717557847$ C, 3.3425282082,1.0947084094,0.4520053864 C, $0.7143478975,-1.432381083,-0.5537595131$ C, $1.3890012712,-2.5066768213,-1.0970283626$ H, 2.4667789492,-2.4726205684,-1.1809421569 C, $0.7140349868,-3.6357443347,-1.5995876299$ C, $1.4013402828,-4.7548823253,-2.1338652346$ H,2.4856106846,-4.7501470902,-2.1376769257 C,0.7076827292,-5.8267621955,-2.632974554 H,1.2416441644,-6.6796513634,-3.0344562582 C, $-0.7070840496,-5.826828379,-2.6329928894$ $\mathrm{H},-1.2409552615,-6.6797677155,-3.0344880049$ C, $-1.4008548523,-4.7550135367,-2.1339013259$ $\mathrm{H},-2.4851256028,-4.7503803376,-2.1377398231$ C,-0.7136682684,-3.6358110905,-1.5996059412 C, $-1.3887541349,-2.5068093938,-1.0970588153$ $\mathrm{H},-2.4665324632,-2.4728569625,-1.1809992844$ C, $-0.7142173461,-1.4324519915,-0.5537665693$ C,-1.4196214872,-0.2173778829,-0.1440750551 C,-3.6579772154,-1.1525392355,0.9306445006 C, $-3.5531075833,-2.5040817672,1.2467245788$ $\mathrm{H},-2.6548499442,-3.0613152079,1.0226689976$ C, $-4.6183753946,-3.1467832748,1.8758825817$ $\mathrm{H},-4.5338063797,-4.1994680698,2.1178780904$ C, $-5.7845944073,-2.4560866962,2.1998942474$ $\mathrm{H},-6.6036305883,-2.9768849476,2.6811377639$ C, $-5.8910791339,-1.0940160607,1.9281743014$ $\mathrm{H},-6.7821055267,-0.5455426189,2.2111296496$ C, $-4.8316777759,-0.4449241109,1.312546615$ C,-4.6336662134,0.9744947957,1.0132291476 C,-5.3876112144,2.1059248778,1.2201346644 H,-6.3811657518,2.0573865039,1.6499466684 C,-4.8156282568,3.3544817415,0.8970553415 H,-5.3758292035,4.2582092912,1.1058477245 C, $-3.5511635471,3.4670566578,0.3510540568$ H,-3.1453426357,4.4540497172,0.1884486006 C,-2.7700771883,2.3128750056,0.0717612969 C,-3.3426465588,1.0943736133,0.4520268946 C, $-2.7101455585,-0.1736492265,0.3478970394$ C, $-0.7258475158,1.0344543779,-0.3660643094$ C,-1.4167886712,2.2616648164,-0.4193733904 C, $-0.7107536169,3.3939715296,-0.9859214241$ C,-1.3847146421,4.4344970076,-1.657515281 H,-2.4595912066,4.3944983386,-1.7509279067 C, $-0.6992132462,5.4562516352,-2.278838068$ H,-1.245114412,6.2285962558,-2.8070813877 C, $0.6986719078,5.4563231724,-2.2788267874$ H,1.2445031844,6.2287235118,-2.8070608605 C, $1.3842656544,4.434636639,-1.6574943953$ H,2.4591490061,4.3947453348,-1.7508843936 C, $0.7103969134,3.3940432199,-0.9859140899$ C, $1.4165419381,2.261806924,-0.4193668495$ C, $0.7257251424,1.0345272777,-0.3660623632$ 